\title{
The role of oligodendrocytes in higher-order circuit functions
}

\section{Dissertation}

\author{
for the award of the degree \\ "Doctor rerum naturalium" \\ of the Georg-August-Universität Göttingen
}

within the doctoral program IMPRS Neuroscience

of the Georg-August University School of Science (GAUSS)

Submitted by

Sharlen Yared Moore Corona

From

Mexico City, Mexico

Göttingen 2018 


\section{Thesis Committee}

Dr. Livia de Hoz (Supervisor)

Department of Neurogenetics, Max Planck Institute of Experimental Medicine

Prof. Klaus-Armin Nave, PhD (Reviewer)

Department of Neurogenetics, Max Planck Institute of Experimental Medicine

Prof. Dr. Mikael Simons (Reviewer)

Institute of Neuronal Cell Biology

Technical University Munich

German Center for Neurodegenerative Diseases

Prof. Dr. Swen Hülsmann

Experimental Neuroanesthesiology, University Medical Center Göttingen

\section{Members of the Examination Board}

Prof. Dr. Tobias Moser

University Medical Center Göttingen

Institute for Auditory Neuroscience \& InnerEar Lab

Camin Dean, Ph.D.

Trans-synaptic Signaling group

European Neuroscience Institute, Göttingen

Prof. Dr. Ralf Heinrich

Department of Cellular Neurobiology

Schwann-Schleiden Research Centre

Date of oral examination: $5^{\text {th }}$, June, 2018 . 
"How does one get ideas? By sheer perseverance to the point of madness" - Charlie Chaplin

to Ma. Teresa Ortega Luna 


\section{Table of contents}

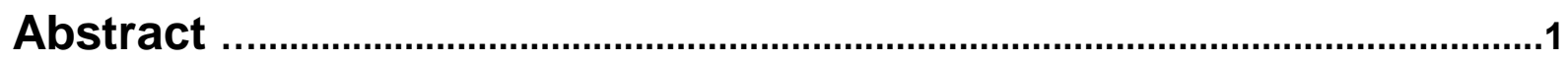

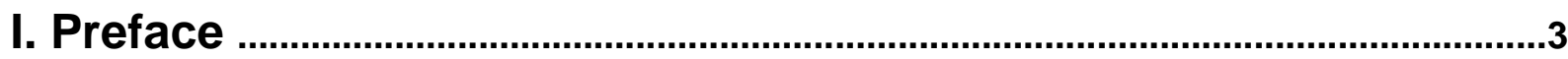

I.I. Myelin: not the textbook 'insulator' ...............................................................

I.II. Myelin in gray-and-white ................................................................................

I.III. The multiple players of conduction velocity regulation ..................................5

I.IV. Myelin pathologies ...........................................................................................6

I.V. Adaptive myelination and the role of oligodendrocytes in shaping neuronal

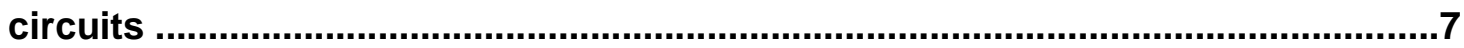

I.VI. Myelin relevance in sensory processing and cognitive functions .................7

I.VII. Myelin basic protein: a handle into myelin manipulation ...............................

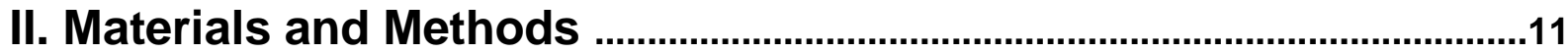

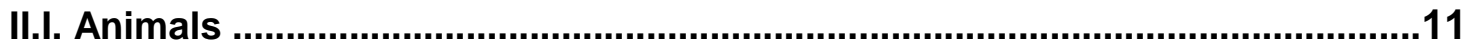

II.I.I Complete dysmyelination model .....................................................11

II.I.II. Partial dysmyelination model .............................................................11

II.I.III. Axo-glial metabolic deficiency model ..............................................12

II.I.IV. Myelinating-cells metabolic deficiency model ................................12

II.II. Wide-use solutions..............................................................................13

II.II.I. Fixatives .............................................................................................13

II.II.II. Anesthetics …............................................................................13

II.III Data management and analysis generalities ..............................................13

II.IV. Electron microscopy .....................................................................................14

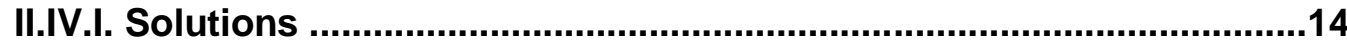

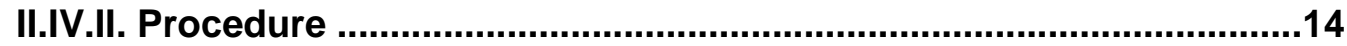

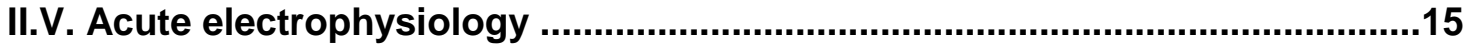

II.V.I. Surgical procedure ............................................................................15

II.V.II. Specifics of experimental animals ...............................................16

II.V.III. Data acquisition ................................................................................17

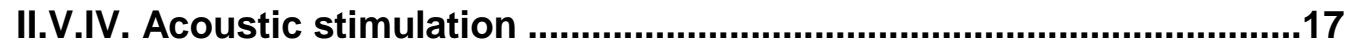

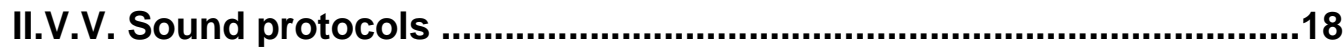

II.V.V.I. Frequency sweep ..........................................................18 
II.V.V.II Click-tracing sweep .18

II.V.V.III. Two broad-band noise pulses separated by a gap...........18

II.V.V.IV. Oddball protocol ............................................................18

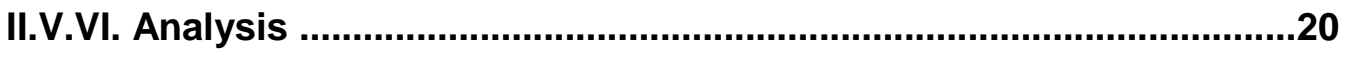

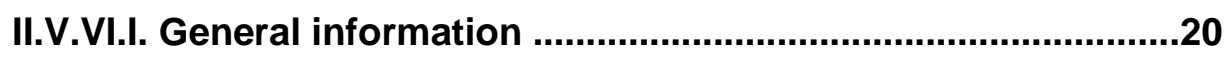

II.V.VI.II. Post-stimulus time histogram ........................................20

II.V.VI.III. Baseline activity .............................................................21

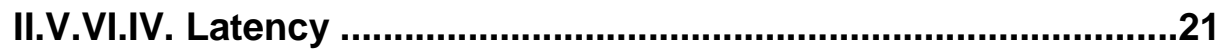

II.V.VI.V. Peak activity ................................................................21

II.V.VI.VI. Temporal reliability .............................................................21

II.V.VI.VII. Temporal acuity ................................................................22

II.V.VI.VII.I. Assessment of the response to the first

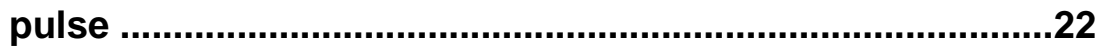

II.V.VI.VII.II. Assessment of gap-detection ............................23

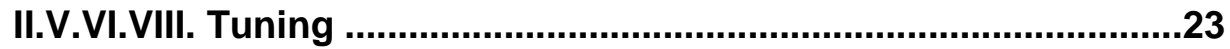

II.V.VI.IX. Stimulus-specific adaptation ...........................................23

II.VI. Auditory brainstem recordings ....................................................................24

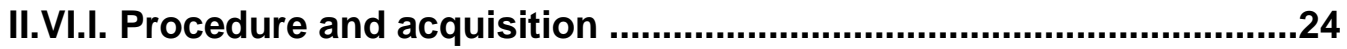

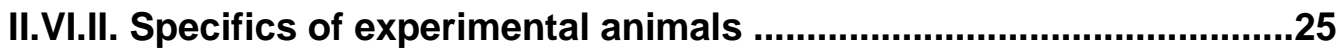

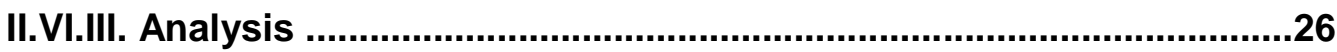

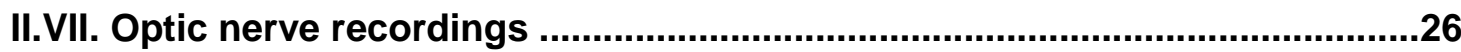

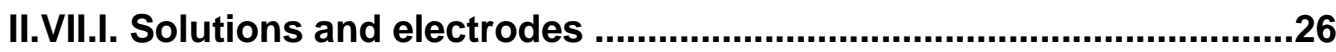

II.VII.II. Optic nerve preparation and stimulation .......................................27

II.VII.II.I. Current-voltage dependence ...........................................28

II.VII.II.II. Ramp stimulation ..........................................................28

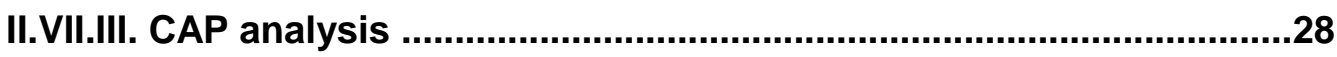

II.VII.III.I CAP amplitude during IV curves ...................................29

II.VII.III.II. Depolarizing and hyperpolarization area ........................29

II.VII.III.III. High-rate stimulation ...................................................29

II.VII.III.IV. Conduction velocity .......................................................30

II.VIII. Axon initial segment length quantification ..............................................30

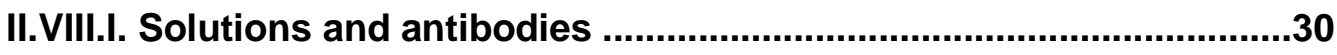

II.VIII.II. Immunostaining ..............................................................................30

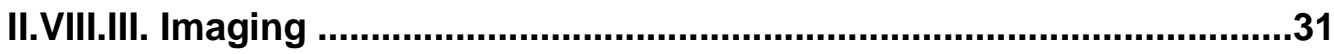




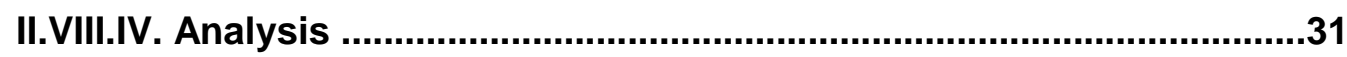

II.IX. Behavioral paradigms .........................................................................31

II.IX.I. Pre-pulse inhibition of the startle reflex using silent gaps ............31

II.IX.I.I. Specifics of experimental animals ....................................32

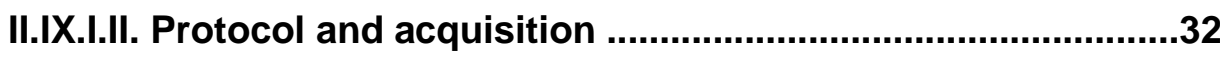

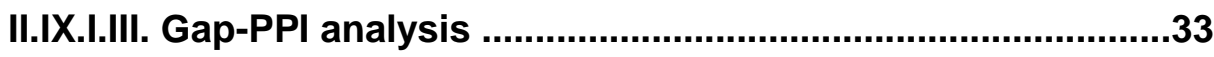

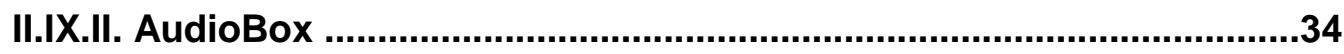

II.IX.II.I. Behavioral system and animal procedure ........................34

II.IX.II.II. Gap detection paradigm ...................................................37

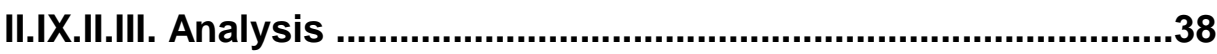

II.X. Paw preference test ...........................................................................................39

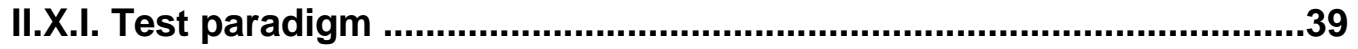

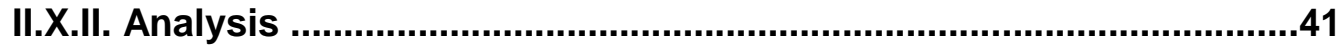

\section{Chapter 1 - Not quite my tempo: auditory abnormalities caused} by dysmyelination ..............................................................................................43

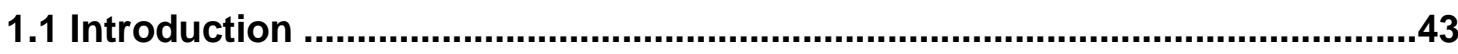

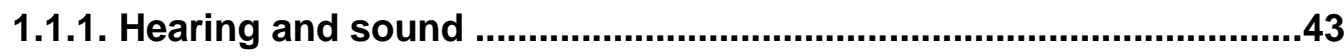

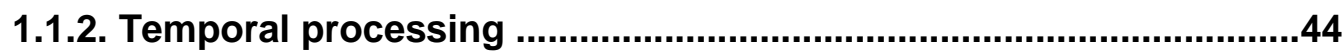

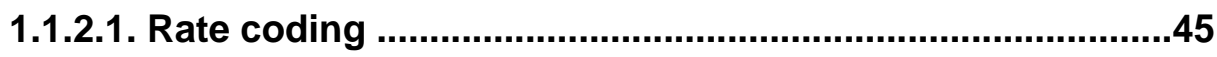

1.1.2.2. Gap detection ......................................................................47

1.1.3. Auditory disturbances related to myelin .........................................48

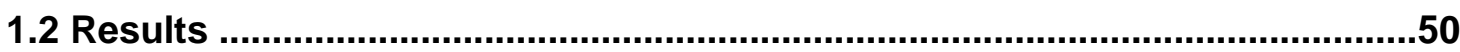

1.2.1. Absence of MBP results in a strong decrease of myelin in different auditory stations. ........................................................................................50

1.2.2. Functional sensory consequences of the total MBP loss in grey and

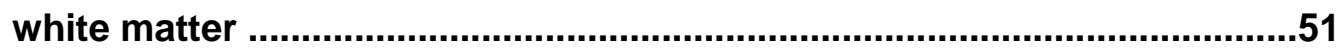

1.2.3. Lack of myelin impairs temporal processing in the auditory cortex 63

1.2.4. Impaired axonal firing at high stimulation rates in $M P^{\text {shi }}$ white

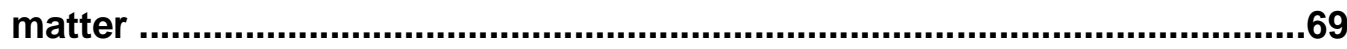

1.2.5. Cortical-specific myelin loss impairs response amplitudes ..........73 
1.2.6. Functional sensory consequences of a partial MBP loss in gray and white matter 79

1.2.7. Mice with partial myelin loss have subtle impairments in temporal acuity and rate coding in the auditory cortex

1.2.8. Partial myelin loss impairs temporal acuity at the behavioral level

1.2.8.1. Gap-elicited pre-pulse inhibition of the acoustic startle reflex in $\mathrm{MBP}^{\text {neo }}$ mice .89

1.2.8.2. Gap-detection using the AudioBox, in $\mathrm{MBP}^{\text {neo }}$ mice .92

1.3. Discussion .96

1.3.1. Functional consequences of the loss of MBP in grey and white matter .96

1.3.1.1. Basic auditory processing is affected with different levels of dysmyelination .96

1.3.1.2. The effects of different levels of dysmyelination in white matter .101

1.3.2. Total myelin loss impairs temporal reliability and acuity in the auditory cortex .103

1.3.2.1. Dysmyelination reduces temporal reliability, measured by rate coding the $\mathrm{ACx}$ 103

1.3.2.2. Dysmyelination reduces temporal acuity, measured by gap-detection in the ACx. .106

1.3.3. Partial myelin loss is enough to impair temporal acuity at the behavioral level .108

1.4. Conclusions 110

\section{Chapter 2 - Oligodendrocyte metabolic impairments affect} axonal function

2.1. Introduction 111

2.1.1. Oligodendrocyte metabolic support 111

2.1.2. The axo-myelinic synapse .113

2.1.3. Other forms of oligodendrocyte metabolic support .113 
2.2.1. Reduced oligodendrocytic trophic support does not impair basic auditory processing in the brainstem ...............................................115

2.2.2. An oligodendrocyte-specific metabolic defect impairs temporal processing in the ACx ........................................................................119

2.2.3. An oligodendrocyte-specific metabolic defect impairs rate coding

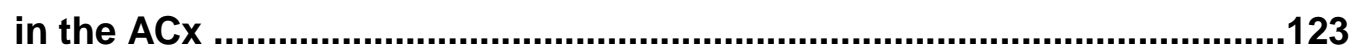

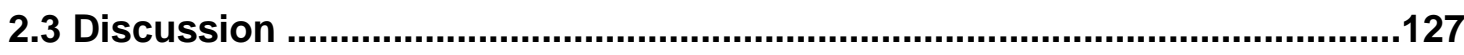

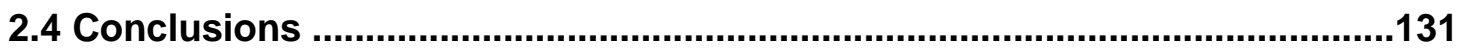

\section{Chapter 3- Myelin and the establishment of behavioral}

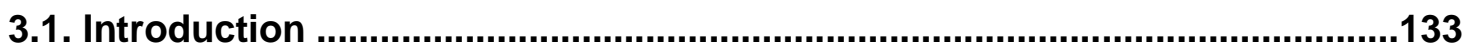

3.1.1. Lateralization of brain function ..................................................133

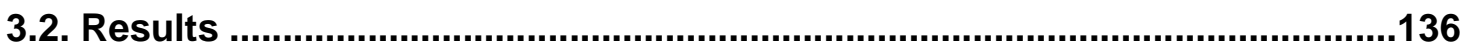

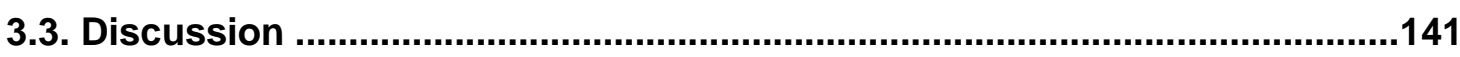

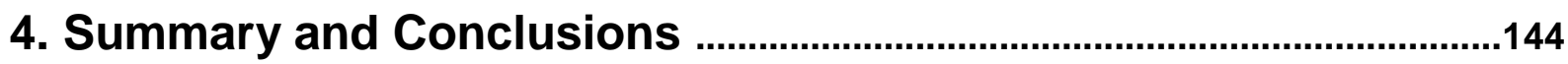

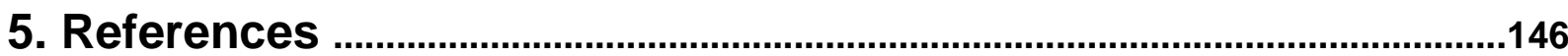

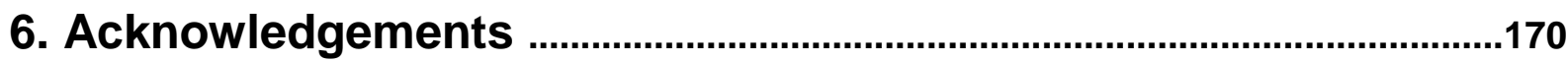

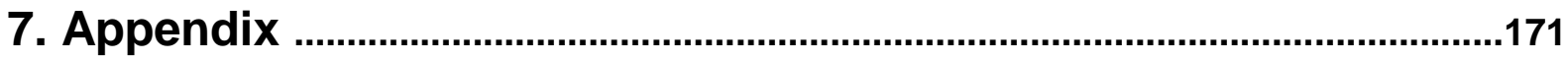

7.1. List of abbreviations .................................................................................171

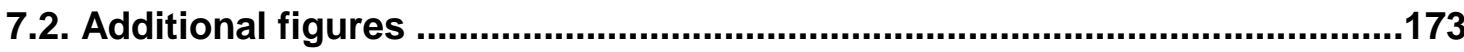

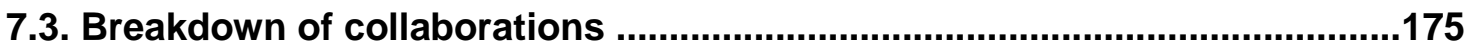

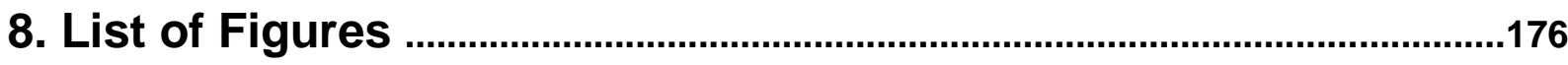

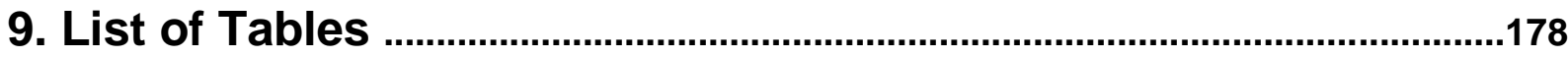

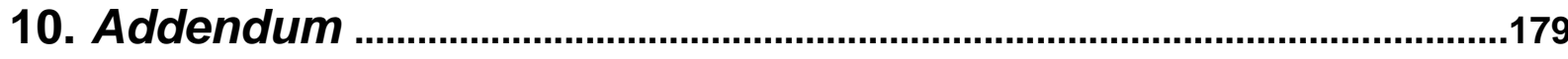

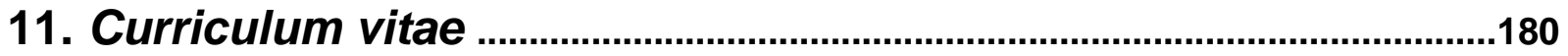




\section{Abstract}

The historical conception of glial cells as the 'glue' that holds the brain together has dramatically changed in the last years. The glia plays an active role in several mechanisms related to information processing in the brain and thus can change and shape circuits during development, adulthood and in an experience-dependent manner. Due to the recently described novel characteristics and roles that these cells have in brain function, we currently face ourselves with the necessity of widening the 'neuro' in neurosciences and give them the appropriate place they deserve.

Focusing on oligodendroglia, the better-known function of these cells is to wrap axons in the central nervous system to facilitate propagation of action potentials along axons. There is also evidence for a role in metabolic support of the axons they wrap that is partially independent of myelination per se. Furthermore, nowadays we know this wrapping process is extraordinarily complex and prone to plastic modifications for the adaptation of information transmission, and vulnerable to injury. The study of sensory and cognitive abnormalities in patients with myelin lesions has provided evidence to understand the function of myelin in information processing. Nevertheless, due to the substantial heterogeneity of these myelin lesions within the study populations, the correlation of specific dysfunctions with an observed phenotype results in a difficult task. Additionally, the complexity of the contact, communication, and maintenance between axons and oligodendrocytes makes it difficult to dissect the partially independent roles that these cells have in information processing.

Studies for the dynamic in vivo assessment of combined neuronal and oligodendrocyte functions are very scarce in the field and have only very recently begun to emerge. In this study, I assessed axonal auditory cortical function in mutant mice with either dysmyelination phenotypes or reduced metabolic axo-glial support to the axons. I found that dysmyelination generated deficits in temporal sound processing known to be essential for speech understanding. The perceptual deficits correlated with defects seen in the spiking activity of cortical cells. Together, they could not be explained by a loss in conduction velocity alone, suggesting that mechanisms that are secondary to the loss of myelin per se, are accounting for the diverse effects observed. Indeed, mice with oligodendrocyte-specific deficits in metabolic processes but relatively minor deficits in myelination showed similar impairments to those found with dysmyelination. A parallel study of the role of myelin in task- 
specific brain lateralization revealed that myelin helps the establishment, but not the maintenance of this circuit function.

Overall my study shows, for the first time, that oligodendrocytes play crucial roles in normal circuit function in high order processing and this role goes beyond to the regulation of conduction velocity. The observations made in this study highlight the importance of glial cells in normal brain function and disease, and emphasize in particular the importance of the cooperative research of neuro-glial interactions. 


\section{Preface}

This section presents a review of the literature relevant to the general topic of myelin functionality in the brain. It highlights the recently discovered roles that oligodendrocytes play in information processing and modulation of neuronal circuits. This general context refers to the principal aim of the project, which is to elucidate how sensory and cognitive processes can be modulated by myelin.

\section{I.I. Myelin: not the textbook 'insulator'}

Myelin is a lipid rich multilayered sheath that wraps axons both in the central nervous system (CNS) and peripheral nervous system (PNS). Evolutionarily speaking, approximately 600 million years ago myelination evolved in vertebrates to allow faster responses to environmental stimuli (Nave and Trapp, 2008), becoming essential for axonal function. In the CNS, it is a membrane extension of the oligodendrocytes (OLs), a type of glial cells that are able to generate many internodes and wrap axons from several neurons at a time. In the PNS, single Schwann cells (SC) wrap individual portions of axons. Myelin has long been defined as the insulator that allows the speeding up of action potential (AP) propagation by helping the formation of excitable axonal domains, called nodes of Ranvier (nR), where saltatory conduction takes place. The $\mathrm{nR}$ are periodic accumulations of voltage-gated ion channels which are anchored to the membrane via a set of specialized cytoskeletal proteins (Nelson and Jenkins, 2017). In this way, myelin increases the membrane resistance and reduces the membrane capacitance (Barrett and Barrett, 1982; Smith, 1994), facilitating the longitudinal internal ion flow along the axon. Ion currents, then, become restricted to the $\mathrm{nR}$, $\sim 0.5 \%$ of the axonal surface, allowing the neurons to save energy (Nave, 2010).

It is known that axons have very high energy requirements (Beirowski, 2013). The $\mathrm{Na}^{+} / \mathrm{K}^{+}$ATPase pump is particularly demanding, and allows the repolarization of the membrane after an AP (Alizadeh et al., 2015). Energy needs are usually met locally through axonal mitochondria (Hollenbeck and Saxton, 2005), which become stationary in potential sites of high energy needs, for example, close to the node of Ranvier (Berthold et al., 1993; Fabricius et al., 1993; Ohno et al., 2011). Neuronal activity reduces the mobilization of mitochondria along the axons and increases the size of stationary mitochondria in nodal/paranodal regions, a process that requires axo-OLs contact (Ohno et al., 2011). In addition, because it was observed that OLs lacking essential myelin proteins (PLP and CNP1) 
had minor defects in myelination but a strong axonal pathology (Edgar et al., 2009; Griffiths et al., 1998; Lappe-Siefke et al., 2003), and that OL ablation had a similar effect (Ghosh et al., 2011; Oluich et al., 2012). The idea that OLs were serving other functions besides myelination was put forward. It was later proven that oligodendrocytes are able to metabolically support axons trough the direct supply of lactate (Fünfschilling et al., 2012; Lee et al., 2012). For all these reasons, it is expected that myelination evolved not only as a mechanism for speeding up conduction velocity but also, for the maintenance of energetic homeostasis in axons; the latter being probably a more ancestral role of glial function (Nave and Trapp, 2008). This suggests that it is plausible that myelin and OLs in different brain regions could be playing different roles, and in structures or cell-types that require higher energetic capabilities, myelin might be even more essential.

\section{I.II. Myelin in gray-and-white}

Recent data shows that the typical structure of myelin in white matter regions might not be representative of that in gray matter. Certain aspects of myelination are also regulated differently. In both peripheral and central white matter, myelin thickness increases in axons with larger diameters (Hildebrand and Hahn, 1978). Also, OLs in white matter can regulate axon caliber (e.g. the optic nerve), using signals that are independent of myelin formation (Sánchez et al., 1996). This effect is confirmed by a change in the axon caliber distributions in the optic nerve of mice that lack compact myelin (Kirkpatrick et al., 2001). Myelination patters in the cortex are more heterogeneous and do not follow the axon caliber rule (Bercury and Macklin, 2015). In the cortex, projection neurons have different myelination patterns along the cortical layers, with more myelin in deep than in the superficial layers (Tomassy et al., 2014). In addition, axons from the deeper layers tend to have more uniform patterns of myelination while superficial axons, surprisingly, present intermittent myelin. This means that long stretches of the axons go without myelin (Micheva et al., 2016; Tomassy et al., 2014) debunking the hypothesis that cortical myelination facilitates conduction velocity along excitatory long-range connections. This feature could help the formation of synaptic connections from other neurons or the cross activation between naked axons in close

proximity (ephaptic activation) (for review, see (de Hoz and Simons, 2015), allowing additional neuronal integration.

Again contrasting what is known for myelin in white matter tracts, it was recently described that half of the myelin found in cortical layer II/III preferentially wraps a very specific 
interneuron type: the parvoalbumin positive $\left(\mathrm{PV}^{+}\right)$basket cells (Micheva et al., 2016). Since the distribution of inhibitory neurons in the cortex is low (20-30\%) and about half of the interneurons are $\mathrm{PV}^{+}$basket cells (Markram et al., 2004), it is quite surprising to see the strong specificity and preferential myelination of this cell type. In addition, it was found that the myelination patterns and myelin composition is different between inhibitory and excitatory cells (Micheva et al., 2016). Similar observations have been replicated in humans (Stedehouder et al., 2017). Because $\mathrm{PV}^{+}$cells have high firing rates, it could be inferred that the main role of myelin in the cortex does not have to do with a homogeneous increase in conduction velocity, but more with the energetic needs of fast-spiking cells (Micheva et al., 2016). In this specific case, we see strong differences in myelination patterns when comparing white matter and gray matter areas, suggesting that myelin might be playing differential roles.

\section{I.III. The multiple players of conduction velocity regulation}

Besides changes in myelin thickness, which could be the simplest explanation for conduction velocity differences, internodal length shifts or sheath additions can result in changes in conduction velocity and AP propagation (Arancibia-Cárcamo et al., 2017; Arroyo et al., 2001; Fields, 2008a; Kimura and Itami, 2009; Seidl, 2014; Tomassy et al., 2014). Typically, larger caliber axons have longer internodes (Friede and Bischhausen, 1982), but this would vary on different CNS areas (Bechler et al., 2015). In the brainstem for example, large caliber axons seem to have shorter myelin sheaths (Ford et al., 2015). On the other hand, nodal length appears more homogeneous in white matter compared to the cortex (Arancibia-Cárcamo et al., 2017). Changes in internodal/nodal lengths could enhance firing synchronization, reduce the dependency of axonal trajectory on AP propagation, or adjust AP velocity in circuits that require coincidence detection from different paths with variable axonal lengths (ArancibiaCárcamo et al., 2017; Seidl, 2014; Seidl et al., 2014). During normal ageing in mice, internodal distances decrease (Lasiene et al., 2009), maybe due to remyelination processes (Smith et al., 1982), suggesting that internodal changes are present during normal ageing (Peters and Sethares, 2003), but can also be used for shaping networks. For example, during demyelination, internodal length reduction facilitates conduction (Waxman and Brill, 1978).

Especially for the auditory system, this process allows the fine tuning of specific pathways, for reliable AP propagation in certain circuits (Ford et al., 2015), and it is essential for the coincidence detection necessary for sound localization (Seidl et al., 2014). In addition, 
heavy myelination of the thalamic projections could help the adjustment of conduction latencies of neurons coming from different path lengths and entering the cortex (Salami et al., 2003). The question arises of whether homogeneous myelination in white matter is serving different functions from inhomogeneous one in gray matter. Could this heterogeneous graymatter myelination reflect a mechanism of plasticity in areas that require more flexible processing? What happens when myelin is affected in specific areas?

\section{I.IV. Myelin pathologies}

A common way of understanding the functionality of a biological process is to study it when it is impaired. In this case, we know that the integrity of myelin is sensitive to several insults. Demyelination can lead to conduction blocks and reduced conduction velocity (El-Badry et al., 2007). This would be explained by the increased internal area on which ions would flow in the axon and the lateral dissipation of charges, leaving the axon with lower currents for depolarization. In addition, membrane capacitance would increase, so the axons would require larger currents to be depolarized (Smith, 1994). During ageing, there is a decrease of the amount of myelinated fibers in white matter and also an increase in myelin degeneration, which leaves the axons unaffected but causes changes in information transfer (Peters, 2009). Changes in white and gray matter myelin are implicated in schizophrenia and other psychiatric disorders ((Chiappelli et al., 2015)for review, see (Fields, 2008a; Haroutunian et al., 2014; Nave and Ehrenreich, 2014; Stedehouder and Kushner, 2017), in early stages of Alzheimer's disease (Bartzokis, 2004; Sachdev et al., 2013), in autism spectrum disorders (Fingher et al., 2017; Miller et al., 2013; Vidal et al., 2006; Wei et al., 2016), and other neurodegenerative diseases (Ettle et al., 2016). Since it is not the intention of this study to address in depth the pathological side, only brief examples are provided here and further. 


\section{I.V. Adaptive myelination and the role of oligodendrocytes in shaping neuronal circuits}

It has been widely reported that OLs express several receptors and ion channels through which they can sense changes in neuronal activity and respond accordingly to their microenvironment's needs (Barres et al., 1988, 1990; Fields, 2008b; Fields and Burnstock, 2006; Káradóttir et al., 2005; Kettenmann et al., 1984; Sontheimer, 1994; Verkhratsky and Kirchhoff, 2007). In addition, OLs can also communicate with axons via exosome export (Frühbeis et al., 2013). The possibility of sensing the neuronal status allows the OLs to perform activities that help the axons. There is in vitro and in vivo evidence that shows that OLs are able to sense the activity of the neurons and respond with distinct patterns of myelination (for review, see (de Hoz and Simons, 2015). Over the last years, it has become evident that $\mathrm{OL}$ proliferation is reduced when the activity of retinal ganglion cell axons is blocked with tetradotoxin (TTX) (Barres and Raff, 1993). Demerens et al, demonstrated that TTX blockage also reduces the number of myelinated segments both in cultured neurons and in vivo (Demerens et al., 1996). Electrically active axons in culture show preferential myelination compared to non-active ones (Wake et al., 2015). In addition, electrical stimulation of hippocampal OLs generates long lasting changes that reduce the conduction velocity of APs (Yamazaki et al., 2007). These effects are not only seen in excitatory cells, since these $\mathrm{PV}^{+}$cortical interneurons show adaptive myelination, which is mainly dependent on changes in the arborization of these neurons upon stimulation (Stedehouder et al., 2017).

In general, OLs have the capacity of sensing their microenvironment and respond with changes in myelination, probably to actively modulate network activity, a new form of plasticity that might be essential for proper sensory and cognitive functions.

\section{I.VI. Myelin relevance in sensory processing and cognitive functions}

We have now enough evidence to say that myelin is a much more dynamic structure than what was previously thought. What is the role of this plasticity in neuronal processing and higher-order brain functions? An increasing amount of studies have recently focused on the role of myelin and OL factors in vivo to understand how myelin changes can actually help and shape certain circuits; nevertheless, they have mainly addressed the topic of motor 
performance (Gibson et al., 2014; McKenzie et al., 2014). My question goes beyond motor processing, aiming to relate specific neural circuit dysfunctions caused by myelin, to sensory and cognitive processing.

Certain activities like juggling (Scholz et al., 2009), learning a new language (Schlegel et al., 2012), or training motor skills (Lakhani et al., 2016; Sampaio-Baptista et al., 2013) can increase myelin content or myelin proteins in the brain during adulthood. Also, activating subpopulations of neurons optogenetically in vivo increases myelination of the active circuits, with an enhancement of the related behavior (Gibson et al., 2014). Conversely, deprivation experiments, like social isolation or exposure to chronic social stress (Lehmann et al., 2017; Liu et al., 2012), reduced maternal interaction (Kikusui et al., 2007) and sleep deprivation (Bellesi et al., 2018) result in a reduction of myelin. In addition, the generation of new OLs is necessary for the fast acquisition of new motor skills (McKenzie et al., 2014). Some of the mechanisms regulating these effects might have to do with plastic changes related to myelin (examples were given in section I.II).

In addition, oligodendrogenesis and changes in myelination are not uniquely seen during development, but actually continue during adulthood ((Hughes et al., 2018) for review, see (Richardson et al., 2011; Zatorre et al., 2012). In cortical white matter, new myelinating OLs are generated, while there is continuous generation of OL progenitor cells in cortical gray matter (Dimou et al., 2008). These changes, associated to the maintenance of plasticity in the cortex, belong to areas where experience-dependent remodeling is seen during adulthood (Chang et al., 2005; Das, 1997; Gilbert and Li, 2012; Long et al., 2018; Schreiner and Polley, 2014; Weinberger, 2012).

Taking this together, OLs are dynamic cells that play a role not only in the proper wiring of neuronal circuits during development, but in the maintenance and facilitation of those circuits during adulthood. 


\section{I.VII. Myelin basic protein: a handle into myelin manipulation}

To understand myelin function, my approach was to manipulate it. A key protein, the Myelin basic protein (MBP), can give us a handle for this manipulation. MBP is located at the cytoplasmic faces of bi-lipid myelin membranes (at the major dense line). MBP is highly conserved in mammals and plays an important role in myelin compaction and formation (Snaidero and Simons, 2017) in the CNS. MBP isoforms arise from alternative splicing and their content in the PNS is lower than in the CNS (5 to $18 \%$ versus $30 \%$ respectively) (Morell and Quarles, 1999). Since the MBP is the second most abundant CNS myelin protein (Boggs, 2006), it is essential for myelination in the CNS where MBP dependent compaction is needed for the continuity of myelination (Snaidero et al., 2014). Without MBP, the CNS lacks myelin.

One key model used in this study was the shiverer mice (MBP ${ }^{s h i}$ ), which completely lacks myelin. They are the most common dysmyelination mouse model used. Due to the importance of this mouse model in my work, here I present a brief summary of the abnormalities seen in $\mathrm{MBP}^{\text {shi }}$ mice.

The mutation causing this phenotype has an autosomal-recessive pattern of heritability (Chernoff, 1981), and it is caused by a deletion in the MBP gene (Kimura et al., 1985; Roach et al., 1983), affecting 5 out of 6 protein-coding exons (Roach et al., 1985). The $\mathrm{MBP}^{\text {shi }}$ mutation results in a severe CNS myelin deficiency due to incomplete myelin sheath formation. The $\mathrm{MBP}^{\text {shi }}$ axons have only a few turns of non-compacted myelin (Kirschner and Ganser, 1980; Popko et al., 1987). They show nodal alterations, with lower frequency of the typical nodal-paranodal structure (Rosenbluth, 1980). They also present erroneous distribution of $\mathrm{K}^{+}$channels along the internodes (Rasband et al., 1999a; Sinha et al., 2006) and reduced $\mathrm{Na}^{+}$channel clusters (Rasband and Trimmer, 2001; Rasband et al., 1999b), together with overexpression of $\mathrm{Na}_{v} 1.2$ channels, which are usually found in non-myelinated axons (Westenbroek et al., 1992). Strikingly, despite the axonal abnormalities and the strong lack of myelin, these mice do not have severe axonal pathology (Griffiths et al., 1998; Inoue et al., 1981; Rosenbluth, 1980). Furthermore, $\mathrm{MBP}^{\text {shi }}$ mice have normal levels of $\mathrm{Na}^{+} / \mathrm{K}^{+}$ ATPase in the brain (Sheedlo et al., 1987). Mice show a generalized tremor starting at around 12 days of age, a phenotype that becomes stronger with time. Hind limbs are more affected, 
especially when engaged in active movement. Mice usually do not survive past 12 weeks of age (Chernoff, 1981).

Enough evidence has been provided to point towards the idea that glial defects can account for important changes at the molecular, cellular and behavioral level in the brain. The new insights into the roles of myelin in sensory function and the interesting patterns of cortical myelination open a door towards the understanding of myelin in circuit formation, stabilization, regulation of conduction velocity, and cell-specific support. In this study, I am interested in elucidating the partially independent roles that myelin plays in sensory and cognitive processing in vivo and to find behavioral correlates that arise from specific OL dysfunctions. OLs are highly complex cells that multitask between axonal domain isolation, nutrition and the maintenance of axonal architecture, and the in vivo study of how they can regulate all these processes in a partially independent manner will help understand their role in network function and provide information regarding task-specific dysfunctions that can be later related to pathologies. I will address the question of the role of myelin in adult high-order cortical processing by studying in vivo, behavioral paw lateralization and auditory system function of mice with different levels of dysmyelination, oligodendrocyte metabolic defects or impaired axo-glial metabolic support. 


\section{Materials and Methods}

\section{II.I. Animals}

All experimental mice used were housed in standard plastic cages, typically with 1 to 5 littermates. They lived under a 12 h / 12 h light / dark cycle (5:30 / 17:30) in a temperature controlled room $\left(\sim 21^{\circ} \mathrm{C} \pm 2{ }^{\circ} \mathrm{C}\right)$, with water and food ad libitum, unless otherwise stated. All animal experiments were in accordance to the local animal care guidelines and approved by the institutional board of animal welfare and the animal welfare office of the local Lower Saxony authorities (LAVES).

\section{II.I.I. Complete dysmyelination model}

A model with severe CNS dysmyelination: the shiverer mice (MBP ${ }^{s h i}$ ) was used (see section I.VII). Since the seizures can be induced by sudden movements on the home cages, or sudden loud sounds, extra care was taken when handling these mutants. All mice were obtained by crossing heterozygote shiverer (MBP ${ }^{\text {shi/t }}$, bread under the C57BL/6N background), because of the shortened lifespan of homozygote animals and reports showing that $\mathrm{MBP}^{\text {shi }}$ mothers have impaired motherhood capacities (Chernoff, 1981). Mice were genotyped by Gudrun-Fricke Bode and Swati Subramanian.

\section{II.I.II. Partial dysmyelination model}

A mouse line with partial dysmyelination was generated in the department of Neurogenetics of the Max Planck Institute of Experimental Medicine, by Wiebke Möbius. These mice were engineered to reduce the MBP expression in a high level, in order to study the effects of strong but not total dysmyelination, without having the behavioral motor phenotypes shown by the $\mathrm{MBP}^{\text {shi }}$ mice. A genetic construct was inserted into the MBP locus that was obtained from the Eucomm consortium (www.mousephenotype.org/about-ikmc/eucomm) (International Mouse Knockout Consortium et al., 2007). The architecture of the construct consists of a splice acceptor site (En2 SA), a lacZ gene, a neomycin (neo) resistant cassette (for selection), and exon 1 of the classical MBP flanked by lox-P sites. Mice were bred either using heterozygotes $\left(\mathrm{MBP}^{n e o /+}\right.$ ), to obtain littermate controls, or by crossing $\mathrm{MBP}^{\text {neo }} \times \mathrm{MBP}^{\text {neo }}$ and separately generating the controls $\mathrm{MBP}^{+/+}$with $\mathrm{MBP}^{+/+}$. This avoided excess of animal use. These animals were bred under the C57BL/6N background. Since the molecular characterization of this model was done by Martin Meschkat, further information on this model 
can be found in (Appendix, Figure 49). Animals were kindly provided, maintained and genotyped by Martin Meschkat and Wiebke Möbius.

\section{II.I.III. Axo-glial metabolic deficiency model}

Mice with a deficiency in lactate transport from the glia to the axons were used. Briefly, they were the same animals as used in (Lee et al., 2012). The result of a reduction in MCT1 would be an uncoupling of lactate transport between oligo or astroglia, towards the axons, which has been reported before (Fünfschilling et al., 2012; Lee et al., 2012). Since total absence of MCT1 is embryonically lethal, heterozygous mice $\left(\mathrm{MCT}^{+/-}\right)$, which present a reduction of $\sim 50 \%$ of MCT1, were used. MCT1 ${ }^{+/}$mice had no obvious behavioral abnormalities at the ages of testing (10-14 weeks of age). Mice were maintained, kindly provided and genotyped by Kathrin Kusch.

\section{II.I.IV. Myelinating-cells metabolic deficiency model}

Mice with an inducible knock down of the hypoxia inducible factor $1 \alpha$ (HIF1 $\alpha$ ) in myelinating glia were created by crossing Hif $1 \alpha^{\mathrm{fl} / f l}$ with PLP-CreER ${ }^{T 2+}$ animals. The Hif $1 \alpha^{\mathrm{fl} / f l}$ mice were generated as described in (Ryan et al., 2000) and were bought from Jackson Laboratories (www.jax.org/). PLP-CreER ${ }^{\mathrm{T} 2}$ mice were generated as described in (Leone et al., 2003) and obtained from Ueli Suter's lab. The mutant animals, upon tamoxifen injection, have a myelinating-glia specific reduction of the Hif1 $\alpha$ factor, an important transcriptional regulator of glycolytic metabolism in response to hypoxia (Majmundar et al., 2010; Semenza, 2012; Yuen et al., 2014). Mild metabolic problems in myelinating glia were expected in these mice. Mice were bred into the C57BL/6N background. Tamoxifen injections started at 8 weeks of age, to avoid interfering with the metabolism of oligodendrocytes during the peak of myelination. Each animal was injected with Tamoxifen (dissolved in oil, 100mg/kg mouse weight per day, i.p.) for 5 consecutive days. Experiments were performed on these animals $\sim 4-6$ weeks after tamoxifen injection. At this age, animals had a mild reduction in myelin proteins, such as MBP and PLP and presented an increase in g-ratios, meaning that myelin was slightly thinner.

Control animals were Hif $1 \alpha^{f / / f l}$ with PLP-CreER ${ }^{T 2-w t}$ and mutant animals were Hif $1 \alpha^{f / / f l}$ with PLPCreER $^{\mathrm{T} 2+}$. Mice were maintained, injected and kindly provided by Iva Tzvetanova, and genotyped by Gudrun Fricke-Bode. 


\section{II.II. Wide-use solutions}

\section{II.II.I. Fixatives}

1) $16 \%$ Paraformaldehye (PFA) stock

$100 \mathrm{ml}$ stock solution was prepared by adding $16 \mathrm{~g}$ of PFA $\mathrm{n} 90 \mathrm{ml}$ distilled $\mathrm{H}_{2} \mathrm{O}$. Solution was heated up at $65^{\circ} \mathrm{C}$ while stirring for $20 \mathrm{~min}$. Droplets of $5 \mathrm{~N} \mathrm{NaOH}$ were added until solution turned clear. Final volume was adjusted to $100 \mathrm{ml}$ with distilled $\mathrm{H}_{2} \mathrm{O}$. After acclimatization of the solution to room temperature, $\mathrm{pH}$ was adjusted to 7.4. Solution was filtered and stored in small aliquots at $-20^{\circ} \mathrm{C}$.

2) $0.2 \mathrm{M}$ Phosphate buffer $(\mathrm{PB})$

Solution A: $27.6 \mathrm{~g} \mathrm{NaH}_{2} \mathrm{PO}_{4} \cdot \mathrm{H}_{2} \mathrm{O}$ in $1 \mathrm{~L}$ distilled $\mathrm{H}_{2} \mathrm{O}$. Solution B: $35.6 \mathrm{~g} \mathrm{Na}_{2} \mathrm{HPO}_{4} \cdot 2 \mathrm{H}_{2} \mathrm{O}$ in $1 \mathrm{~L}$ distilled $\mathrm{H}_{2} \mathrm{O}$. Solutions were kept at room temperature, and mixed fresh every time in a ratio 1:4 (A:B).

3) $4 \%$ PFA working solution

$100 \mathrm{ml} 0.2 \mathrm{~PB}, 1.6 \mathrm{~g} \mathrm{NaCl}(\mathrm{w} / \mathrm{v})$ and $50 \mathrm{ml}$ 16\% PFA. Final volume was adjusted to $200 \mathrm{ml}$ with distilled $\mathrm{H}_{2} \mathrm{O}$.

\section{II.II.II. Anesthetics}

2\% Avertin (2,2,2-Tribromoethanol 97\%) (Sigma-Aldrich, T48402) and 2\% 2-Methyl-2-butanol (>99\%, Sigma-Aldrich) were dissolved in saline solution ( $\mathrm{NaCl} 0.9 \%$, Braun, Germany) preheated at $35^{\circ} \mathrm{C}$. Solution was stirred until dissolved. The anesthetic was then filtered using a Millex-GP syringe-driven filter unit $(0.22 \mu \mathrm{m}$, Merck KGaA, Germany).

\section{II.III. Data management and analysis generalities}

All the analysis performed in this study was done in a Matlab (The Matworks ${ }^{\circledR}$, USA) environment and custom-made analysis routines. For the statistical analysis, normality was tested using a Shapiro-Wilk test or a one-sample Kolmogorov-Smirnov test. In the case of one-to-one comparisons, either t-tests or non-parametric rank sum tests were performed depending on the normality. The rest of the statistical analysis was done assuming normality and using multiple comparisons ANOVAs for the data that represented individual animals, and assuming non-normality for the data that was presented as individual files, where a Kruskal-Wallis test was used instead. In all cases the corresponding significance values were expressed as follows: n.s. $p>0.05,{ }^{*} p \leq 0.05,{ }^{* *} p \leq 0.01,{ }^{* * *} p \leq 0.001,{ }^{* * * *} p \leq 0.0001$. 


\section{II.IV. Electron microscopy}

\section{II.IV.I. Solutions}

1) Karlsson-Schultz fixative was prepared with $4 \%$ PFA and $2.5 \%$ (v/v) Glutaraldehyde in 0.1 M Phosphate buffer.

2) Epon (Richardson et al., 1960) was prepared by mixing $171.3 \mathrm{~g}$ Glycidether 100 (Serva), $115 \mathrm{~g}$ Dodecenyl succinic anhydride (DDSA, Serva), $89 \mathrm{~g}$ Methylnadic anhydride (MNA, Serva) for 10min. Then $6.5 \mathrm{ml} \mathrm{DMP-30} \mathrm{(Serva)} \mathrm{was} \mathrm{added} \mathrm{and} \mathrm{stirred} \mathrm{for} 20 \mathrm{~min}$.

\section{II.IV.II. Procedure}

For electron microscopy (EM), 7-11 week old $\mathrm{MBP}^{\text {shi }}$ and $\mathrm{MBP}^{+/+}$mice were anaesthetized with Avertin (250 mg/Kg body mass, i.p.) (Sigma Aldrich, T48402), and perfused with $5 \mathrm{ml}$ of Hanks balanced salt solution (HBSS, PAA laboratories, Pasching, Austria) followed by fixative according to (Karlsson and Schultz, 1965) using a Heidolph PD5201 peristaltic pump. The brains where carefully dissected and $200 \mu \mathrm{m}$ coronal sections were cut using a Leica VT1200S Vibratom (Leica Microsystems, Wetzlar). The region of interest was punched out of the section by using a 2mm Harris Uni-core Punch and embedded in Epon (Serva) after postfixation with $2 \% \mathrm{OsO}_{4}$ (Science Services, Munich, Germany) and dehydration with ethanol and propylene oxide using an automatic system (EMTP Leica Microsystems, Wetzlar). Epon embedded samples were cut with the microtome (Ultracut S, Leica). Diamond knifes (Histo $45^{\circ}$ and Ultra $45^{\circ}$, Diatome Biel $\mathrm{CH}$ ) were used to prepare semi-thin (500 nm) and ultra-thin $(50 \mathrm{~nm})$ sections. Semi-thin sections were collected onto a glass slide and dried on a $60^{\circ} \mathrm{C}$ hot plate to verify the ROI by using a Leica Dialux 20 light-microscope. Ultra-thin $\mathrm{nm}$ sections were placed on a 100 mesh hexagonal copper Grids (Gilder Grids Ltd. Grantham UK) coated with "Formvar" (Plano Wetzlar) and stained with Uranylacetat (SPI-Chem West Chester,USA) and lead citrate (Merck, Darmstadt) according to (Reynolds, 1963). Ultra-thin sections were analyzed by using a Zeiss EM912 Electron-Microscope (Zeiss, Oberkochen, Germany) and digital pictures were obtained using the wide-angle dual speed 2K-CCDCamera (TRS, Moorenweis, Germany). Axon Analysis was performed using ImageJ software (national Institutes of Health, Bethesda, USA). All EM procedures shown in this thesis were performed by Torben Ruhwedel at the Max Planck Institute of Experimental medicine, under the guidance of Wiebke Möbius. 


\section{II.V. Acute electrophysiology}

\section{II.V.I. Surgical procedure}

For acute electrophysiological recordings, mice were anesthetized with Avertin mixture (see section II.II.II). An initial dose of $250 \mathrm{mg} / \mathrm{Kg}$ body mass (i.p.) was followed by supplemental doses ( $\sim 1 / 6$ of the initial dose) approximately every 20-30 minutes, based on the presence of the toe-pinch reflex. Once the areflexic state of anesthesia was reached, mice were placed onto a stereotaxic frame (Kopf Inc., Germany), fixed with blunt ear-bars and maintained at $\sim 36^{\circ} \mathrm{C}$ by a rectal-based temperature controller (World Precision Instruments, ATC 1000). After cleaning the skin over the skull with ethanol $70 \%$, the skull was exposed and cleaned of adherent tissue with a scalpel and hydrogen peroxide. A metal screw $(2 \mathrm{~mm} \times 1 \mathrm{~mm}, \mathrm{M} 1 \times 1$, Germany) was inserted into the right parietal cortex and served as ground. To access the left auditory cortex (ACx), a metal post was glued frontal to lambda with dental cement (Unifast, TRAD), such that the skull could now be held through this post and the ear bars removed. This allowed for the rotation of the mouse's head in a $45^{\circ}$ angle in order to raise the temporal bone and perform the craniotomy. The muscle temporalis was detached from the skull and a $4 \times 2 \mathrm{~mm}$ craniotomy was performed using a dental drill (World Precision Instruments, Omnidrill3, tip \#7). This was done following the contour delimited rostral and ventrally by the squamosal suture, dorsally by the temporal ridge, and caudally by the lambdoid suture (Figure 1).
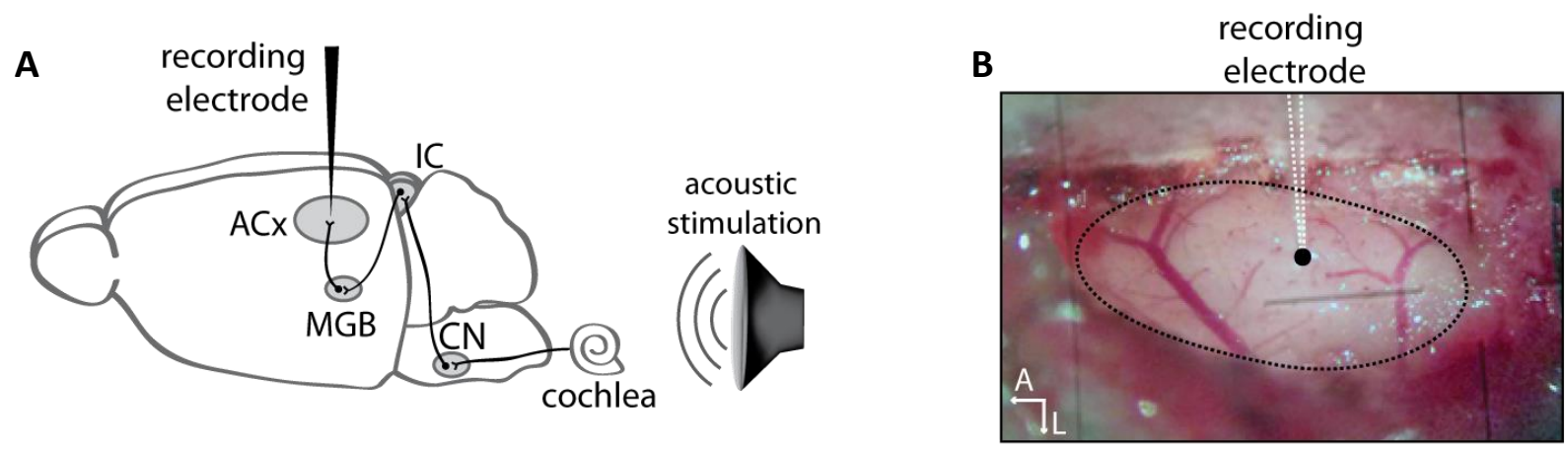

Figure 1. Schematic overview of the auditory pathway and the recorded areas.

Recordings were made from the left auditory cortex or the left inferior colliculus while acoustic stimulation was played on the contralateral ear of the anesthetized mice. A) Schematic representation of the main stations in the auditory pathway. When an acoustic stimulus reaches the cochlea, the air pressure provokes the vibration of this organ, further generating the depolarization of hair cells, which send the signal to the auditory nerve. The nerve synapses into the cochlear nucleus $(\mathrm{CN})$, the first auditory station. The signal is further transmitted through the inferior colliculus (IC), and the medial geniculate body (MNG), until it reaches the auditory cortex (ACx). This is where recordings took place as indicated by the recording electrode. $B$ ) Example image of the craniotomy performed over the ACx and the electrode position. 
Electrophysiological recordings were made using glass coated electrodes: either tungsten with an impedance of $900 \mathrm{M} \Omega$ (AlphaOmega, Germany) or tungsten/platinum with impedances between 1.5 to $2 \mathrm{M} \Omega$ (Thomas Recordings, Germany). After the craniotomy, a drop of saline ( $\mathrm{NaCl} 0.9 \%$, Braun, Germany) was used to clean the surface of the brain. The head of the mouse was then rotated further such that the electrode was inserted through the dura mater perpendicular to the surface of the cortex to reach progressively deeper layers. The selection of insertion area was done following the vein patterns reported in (Joachimsthaler et al., 2014) to aim for the primary fields of the auditory cortex. Responses were characterized by onset latency and shape. A micromanipulator (Kopf, Inc., Germany) was used to advance in steps of $100 \mu \mathrm{m}$. Typically, lowering the electrode through the first $300 \mu \mathrm{m}$ was done with relatively high speed. After the electrode insertion, the brain was allowed to stabilize for 10-20 minutes before continuing with the procedure. After this period, electrode penetration was confirmed and if necessary the dura mater was broken with a 30 gauge $1 / 2$ " needle tip ( $20 \%$ of the recordings).

\section{II.V.II. Specifics of experimental animals}

All the mice used for acute electrophysiology are summarized in the table below. Both males and females were pooled together for analysis.

Table 1. Summary of numbers and characteristics of the experimental animals used for acute electrophysiology recordings.

\begin{tabular}{|c|c|c|c|c|c|c|}
\hline Mouse line & $\begin{array}{c}\text { \# controls } \\
(\uparrow / \delta)\end{array}$ & $\begin{array}{c}\text { \# mutants } \\
\left(\uparrow /{ }^{\wedge}\right)\end{array}$ & $\begin{array}{c}\text { Age } \\
\text { (weeks) } \\
\text { mean/std }\end{array}$ & $\begin{array}{l}p \text { value } \\
\text { age }\end{array}$ & $\begin{array}{l}\text { Mean rec. } \\
\text { depth }(\mu \mathrm{m}) \\
\text { mean/std }\end{array}$ & $\begin{array}{l}\text { p value } \\
\text { rec. depth }\end{array}$ \\
\hline $\mathrm{MBP}^{s h i}(\mathrm{ACx})$ & $10(6 / 4)$ & $13(9 / 4)$ & $8.3 / 2.42$ & 0.92 & $403.9 / 61.6$ & 0.71 \\
\hline $\mathrm{MBP}^{\text {shl }}(\mathrm{IC})$ & $8(4 / 4)$ & $8(4 / 4)$ & $10.7 / 1.97$ & 0.53 & $389.3 / 72.4$ & 0.15 \\
\hline $\mathrm{MBP}^{n e o}(\mathrm{ACx})$ & $8(8 / 0)$ & $8(5 / 3)$ & $12.5 / 2.98$ & 0.78 & $350 / 60.4$ & 0.52 \\
\hline $\mathrm{MBP}^{\text {neo }}(\mathrm{IC})$ & $6(4 / 2)$ & $4(2 / 2)$ & $10.9 / 2.52$ & 0.98 & $344.4 / 81.2$ & 0.58 \\
\hline MCT1(ACx) & $8(3 / 5)$ & $6(3 / 3)$ & $13.1 / 1$ & 0.094 & $378.2 / 37.3$ & 0.32 \\
\hline Hif1a(ACx) & $7(1 / 6)$ & $6(2 / 4)$ & $12.8 / 1.42$ & 0.17 & $340 / 36.3$ & 0.20 \\
\hline $\operatorname{MBP}^{e m x}(A C x)$ & $6(3 / 3)$ & $6(5 / 1)$ & $13.8 / 0.8$ & 0.2 & $360 / 53.2$ & 0.25 \\
\hline
\end{tabular}




\section{II.V.III. Data acquisition}

Recording and visualization of the data was made using the Cheetah Data Acquisition System software (Neuralynx, USA). Electrophysiological signals were acquired at $32 \mathrm{kHz}$ sampling rate, pre-amplified (HS-36-Led, Neuralynx, USA) and sent to an acquisition board (Digital Lynx 4SX, Neuralynx, USA). Voltage values were acquired using a bandpass filter (high-pass: 0.1 or $200 \mathrm{~Hz}$; low-pass: $9000 \mathrm{~Hz}$ ) and stored for offline analysis. For multi-unit activity (MUA), voltage values were high-pass filtered at $350 \mathrm{~Hz}$ using a zero phase filter. MUA detection was done using a threshold that was 6 times the mean absolute deviation from the median of the filtered voltage traces. In addition, only spikes of at least $50 \mu \mathrm{V}$ in amplitude were considered in the analysis.

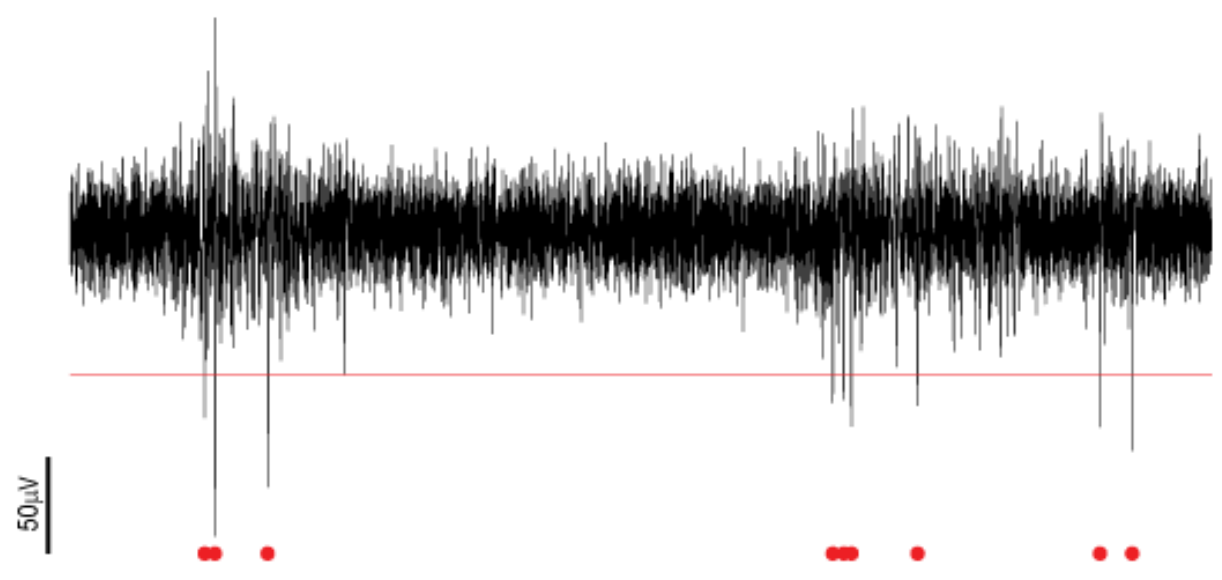

Figure 2. Scheme of the MUA detection using a thresholding method.

A threshold (red line) corresponding to 6 times the mean absolute deviation from the median is applied to the filtered voltage traces (black) for multi-unit spike detection. Additionally, only those spikes that are larger than $50 \mu \mathrm{V}$ are considered in the analysis. Final depiction of the considered spikes is seen with red dots.

\section{II.V.IV. Acoustic stimulation}

All sounds were synthesized in Matlab at a sampling rate of $200 \mathrm{kHz}$. The sounds were delivered by an USB audio interface (Octa-capture, Roland, USA), amplified with a Portable Ultrasonic Power Amplifier (Avisoft, Germany) and played in a free-field ultrasonic speaker (Ultrasonic Dynamic Speaker Vifa, Avisoft, Germay), located $\sim 13 \mathrm{~cm}$ from the right ear of the mouse. Sound intensity was calibrated with a Bruël \& Kjaer (4939 1/4" free field) microphone. The exact time ( $\pm 6 \mu \mathrm{s})$ of sound onset elicited a pulse in the recording acquisition system that was used to synchronize the sound presentation with the neuronal recordings. Sounds were 
either pure tones, broad band noise (BBN, a wide combination of simultaneously played frequencies), or clicks (a very short sound pressure wave).

\section{II.V.V. Sound protocols}

\section{II.V.V.I. Frequency sweep}

Pure tones and tones presented at different intensities were uses to measure tonotopy. Pure tones of variable frequencies and intensities were presented (frequencies ranged from 2 to 31 $\mathrm{kHz}$, and intensities between 0 and $80 \mathrm{~dB}$ ) (Figure 3-A). Each tone had duration of $30 \mathrm{~ms}$, an on/off ramp of $5 \mathrm{~ms}$, and the ITI was $500 \mathrm{~ms}$. Five repetitions of each frequency/intensity combination were presented in a random order.

\section{II.V.V.II. Click-tracing sweep}

To assess temporal reliability upon continuous stimulation, a click-tracing protocol was used. This consisted of a set of 10 clicks presented at different rates (2 to $20 \mathrm{~Hz}$ ) (Figure 3-B). 10 repetitions of each stimulus were played in a random order.

\section{II.V.V.III. Two broad-band noise pulses separated by a gap}

Silent gap-detection in an otherwise continuous broad-band noise (BBN) was used to assess temporal acuity. This sound consisted of a BBN of $200 \mathrm{~ms}$ as the initial pulse, followed by different gap lengths $(0,0.5,1,2,3,4,5,7,10,20,50$ and $100 \mathrm{~ms})$, which were then followed by a second BBN pulse (50 ms) (Figure 3-C). The on/off ramps were $1 \mathrm{~ms}$ long. Each pulsegap-pulse combination was repeated 10 times in a random order. Since using different frequencies for the pulses in the gap-detection task represents a spectral discontinuity in addition to the actual temporal phenomena of detecting a gap, only noise based sounds for gap-detection were used.

\section{II.V.V.IV. Oddball protocol}

Pure tones were used to measure stimulus specific adaptation (SSA), a property of the auditory system for deviant detection. This consisted on the presentation of two pure tones $\left(f_{1}\right.$ and $f_{2}$ ) with a different appearance probability, such that one was rare in the sequence (deviant) and the other one appeared often (standard) (Figure 3-D). Tones were separated in the frequency range by $10 \%(\Delta f=1.1) . f_{1}$ and $f_{2}$ were centered on the best frequency of the MUA at each recording site. The calculation of $\Delta \mathrm{f}$ was made as seen in Equation 1. 


\section{Equation 1}

$$
\Delta f=\frac{f_{1}-f_{2}}{f_{1} f_{2}}
$$

A) Frequency sweep

B) Click-tracing sweep

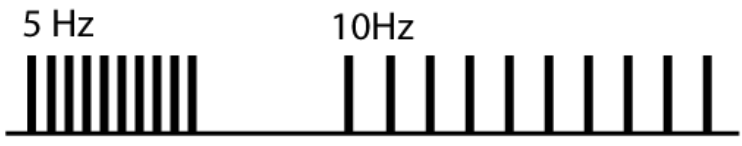

\section{C) Noise-gap-noise pulses}

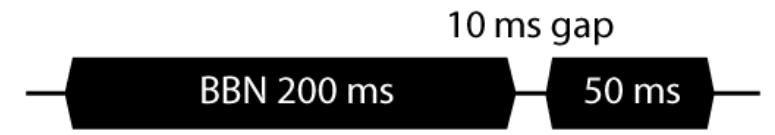

\section{D) Oddball protocol}

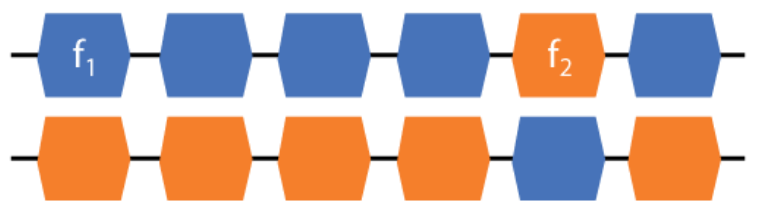

Figure 3. Scheme of the sound protocols used for acute auditory cortex recordings.

A) Frequency sweeps consisted of pure tones $(2-31 \mathrm{kHz})$ presented at different intensities to assess frequency tuning. B) Click-tracing sweeps were presented at different rates (e.g. 5 $\mathrm{Hz}$ ). A set of 10 clicks comprised the click train used to assess firing reliability. C) Broad band noise pulses interrupted by gaps were used to study temporal acuity. D) An oddball paradigm to test stimulus specific adaptation consisted in two pure tones presented continuously. One tone $\left(f_{1}\right)$ was the standard sound and the other one $\left(\mathrm{f}_{2}\right)$ was the deviant. The protocol was also presented in the reverse order. 
The typical intensity at which the sounds were played was 70-80 dB. Three different probabilities of presentation of the deviant sound were used (5, 10 and $20 \%)$. The lower the probability, the greater deviance detection the neurons present. All stimuli used for the analysis were presented at a rate of $3 \mathrm{~Hz}$. Each combination of stimuli ( $\Delta \mathrm{f}$ and percentage of appearance) was presented in a random order, approximately 300-500 times. The number of pulses played depended on the deviant probability, such that 25 deviant tones were played.

\section{II.V.VI. Analysis}

\section{II.V.VI.I. General information}

Typically, the responses of all recorded files from one animal were averaged together and taken as the response of one animal, unless specific analysis states that comparisons were done using the individual files.

\section{II.V.VI.II. Post-stimulus time histogram}

The post-stimulus time histogram (PSTH) was obtained by summing the spikes evoked by the 10 repetitions of the given stimulus. Each PSTH was obtained using different time windows, depending on the sound protocol. See Figure 4, for example.

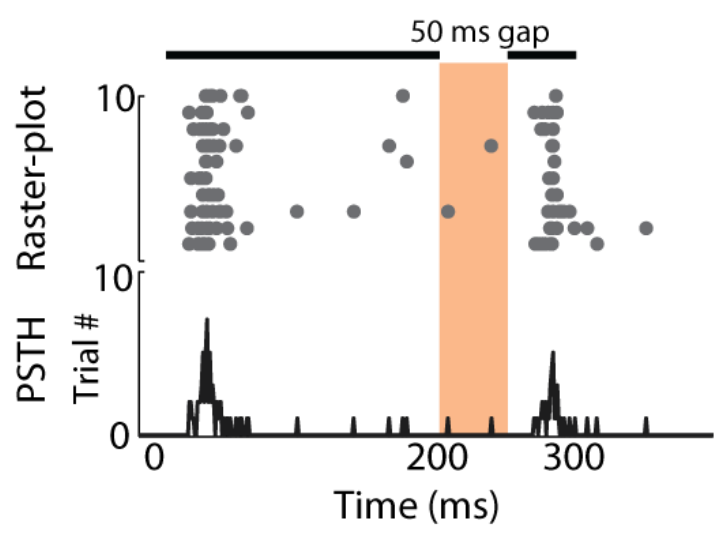

Figure 4. Example of the generation of a PSTH.

A noise-gap-noise sound was presented (depicted by the upper black bars). Spiking activity (individual gray dots) is seen upon sound presentation. A gap (50 ms, depicted by an orange shaded bar) was presented and then a second noise elicited a second burst of responses. PSTH is the sum of all spikes happening at every ms. 


\section{II.V.VI.III. Baseline activity}

A time window was obtained before the sound onset. Depending on the analysis, this window typically varied between 50-200 $\mathrm{ms}$. In all recording files, the comparison between baseline activity and evoked activity was performed in order to consider only those recordings that had significant responses compared to baseline. Significance was obtained with a paired t-test and $\mathrm{a} p \leq 0.05$.

\section{II.V.VI.IV. Latency}

Quantification of spike latency was done using the response to the first click of the clicktracing protocol. The mean of the first click latencies at 5,10 and $20 \mathrm{~Hz}$ was then reported as the latency measurement. The PSTH of a specific stimulus rate was obtained using a $70 \mathrm{~ms}$ window, starting at stimulus onset. The latency was the first time-point in the PSTH that was 1.5 times above the baseline activity (sum of spikes in a $70 \mathrm{~ms}$ window preceding sound onset). Only those recording sites with latencies between 10 and $35 \mathrm{~ms}$ (onset response) were considered in the analysis.

\section{II.V.VI.V. Peak activity}

For quantification of the response amplitude, the peak of the PSTH (as described in section II.V.VI.II) was obtained using a $70 \mathrm{~ms}$ window. Similar as in the latency quantification, response amplitudes (spike count) at 5,10 and $20 \mathrm{~Hz}$ of the first click were averaged per animal. PSTHs were smoothed always using the same smoothing level, for illustration purposes.

\section{II.V.VI.VI. Temporal reliability}

Temporal reliability was assessed with the measurement of spike synchrony, using the clicktracing sweep protocol. For this analysis, circular statistics were implemented (Ashida et al., 2010) to measure the vector strength, and the response to the first click from the train of 10 clicks was discarded as suggested by (Sakata, 2016). Briefly, the times of all the spikes evoked by the remaining 9 clicks were converted to latency $\left(\tau_{i}\right)$ respective to the previous click stimulus (each click becomes $0 \mathrm{~ms}$ ). The times were then converted into radians within a circular vector with phase $\left(\theta_{i}\right)$ between 0 and $2 \pi$, with a phase duration that matched the interclick interval for that rate stimulus. Additionally, a series of 6 sliding windows were selected to measure the spike phases in steps of 4 clicks. The sliding windows comprised clicks 2-5, 3-6, 
4-7, 5-8, 6-9, and 7-10. This process was performed for every rate. The spike phases were then expressed as seen in

Equation 2.

\section{Equation 2}

$$
\theta_{i}=2 \pi \frac{\bmod \left(\tau_{i}, \rho\right)}{\rho}
$$

Where $\tau_{\mathrm{i}}$ was the latency of each spike, $\theta_{\mathrm{i}}$ the phase representation of each spike, and $\rho$ was the phase of the stimulus presented (for example for a click train at $5 \mathrm{~Hz}, \rho=\frac{1}{5}, \rho=0.2$ ). Then, a value of the vector strength (VS) of phase locking was obtained by:

\section{Equation 3}

$$
V S=\frac{\sqrt{\left(\sum_{i} \cos \theta_{i}\right)^{2}+\left(\sum_{i} \sin \theta_{i}\right)^{2}}}{n}
$$

The total number of spikes (n) was used to normalize the resultant vector. To calculate the percentage of spike synchrony, the VS was binned in 30 bins from 0 to $2 \pi$, and the maximum spike count of the bin with the maximum synchronization in the first half of the vector $(\pi)$, was taken. This spike count was expressed in terms of percentage of the total amount of spikes that comprised the specific window analyzed.

\section{II.V.VI.VII. Temporal acuity}

Temporal acuity was studied using the noise-gap-noise pulses protocol, which assesses gapdetection. Only those recordings that had a significant response to the first pulse, across all gaps, compared to baseline, were taken (as described in section II.V.VI.III, using a $100 \mathrm{~ms}$ window). Per animal, all files recorded across different positions in the ACx were averaged and only those animals that had a significant response to $100 \mathrm{~ms}$ gap were considered in the analysis (100 ms window).

\section{II.V.VI.VII.I. Assessment of the response to the first pulse}

To compare the responses to the first sound, a window of a window of $100 \mathrm{~ms}$ which contained the response to the first pulse (pre-gap) before the selected gaps $(0.5,1,2,3,4$ and $5 \mathrm{~ms}$ ) was taken and averaged across the responses at the selected gaps, since this 
initial response is independent of the silent gap presented afterwards. To avoid the effects of latency shifts, the PSTHs of both groups were centered equally for comparison. The spiking activity was then compared between control and mutant animals using an ANOVA.

\section{II.V.VI.VII.II. Assessment of gap-detection}

The analysis aimed to assert whether the second pulse after each gap length had elicited a significant response to the second pulse. To compare the amplitude of the PSTH across groups, first the evoked PSTH over a window of $100 \mathrm{~ms}$ from stimulus onset was constructed. To account for the effects of latency shifts, specially seen in mutant animals, the PSTHs of all animals were aligned to the peak of the mean PSTH and shifted accordingly for different gap lengths. Peak amplitudes were compared over a window of $21 \mathrm{~ms} \mathrm{(10} \mathrm{before} \mathrm{and} 10$ after the maximum peak for both groups) across the different gaps. In the mutant animals where a significant reduction in the pre-gap pulse was seen, a different analysis was performed to assess the level of gap-detection. A $50 \mathrm{~ms}$ window of baseline activity before the pre-gap pulse was taken and compared using a paired t-test with a $50 \mathrm{~ms}$ window that corresponded to the presentation of the post-gap pulse. P-values $\leq 0.05$ were considered as significant gapdetection. All p-values obtained per recording file were plotted against the gap-length. Higher p-values meant better gap-detection.

\section{II.V.VI.VIII. Tuning}

To address changes in frequency tuning in the auditory cortex, a value of the tuning curves width was obtained by adding the amount of spikes that were elicited by specific tones and levels in a matrix-like fashion using a window of $60 \mathrm{~ms}$ after stimulus onset. These values were then converted to data points representing the amount of points where there was spiking activity in an area. The best frequency (BF) was measured as the tone that elicited the maximum amount of spikes for sounds of $80 \mathrm{~dB}$.

\section{II.V.VI.IX. Stimulus-specific adaptation}

For the analysis of stimulus specific adaptation, the SSA index is calculated as follows:

\section{Equation 4}

$$
S S A i=\frac{\partial s-\rho s}{\partial s+\rho s}
$$

Where $\delta$ s represents the response evoked by the deviant sound and $\rho$ s the standard sound. 


\section{II.VI. Auditory brainstem recordings}

Auditory brainstem responses are small sound-evoked potentials measured on through the electrodes located over the skull. They reflect the activity of different early auditory centers, typically up to the inferior colliculus (IC). They are widely used for audiological measurements in humans and experimental models (Dehmel et al., 2012; El-Badry et al., 2007; Ito et al., 2004; Land et al., 2016; Lasky et al., 1999; Spitzer et al., 2015; Verhulst et al., 2016; Willott, 2006).

\section{II.VI.I. Procedure and acquisition}

Prior to electrode placement, all mice were treated according to the following protocol, except the $\mathrm{MBP}^{\text {neo }}$ that followed the procedure reported in (Jung et al., 2015). Mice were anesthetized with an intraperitoneal injection of Avertin mixture (250 mg/Kg, i.p). Anesthesia levels were monitored with the pinch-toe reflex and additional doses of anesthetic were administered accordingly in animals anesthetized with Avertin mixture (typically every 20-30 min using $\sim 1 / 6$ of the induction dosage). Animals were kept under controlled temperature $\left(\sim 36^{\circ} \mathrm{C}\right)$ using a rectal temperature controlled heating pad (World Precision Instruments, ATC 1000). Subdermal needles (BD Microlance, 30G 1/2”, 0.3×13 mm) were pierced through the skin of the mouse and used as electrodes, after the insulation was taken out with a scalpel. The electrodes were reused for a maximum of 4 times. The active (+) electrode was placed at the vertex of the skull, the reference electrode (-) below the left pinna, and a third electrode at the base of the tail for active shielding. Stimuli were ipsilaterally delivered through a free field electrostatic speaker (Avisoft, Germany) placed $\sim 9 \mathrm{~cm}$ from the left ear. The recordings were conducted in a sound-attenuated and anechoic room. The difference in potentials was amplified 10000 times using a custom made amplifier. A National instruments shielded I/O connector block (NI SCB-68) was interfaced with Matlab for the stimulus presentation and data acquisition (50 $000 \mathrm{~Hz}$ sampling rate). Sound stimuli were digitally generated using Matlab. Square waveforms for clicks (0.03 ms duration / 50 ms ITI / $20 \mathrm{~Hz}$ presentation rate) were presented at different sound pressure levels $(0,30,40,45,50,55,60,70$ and $80 \mathrm{~dB})$ and were randomized throughout the recording session. 


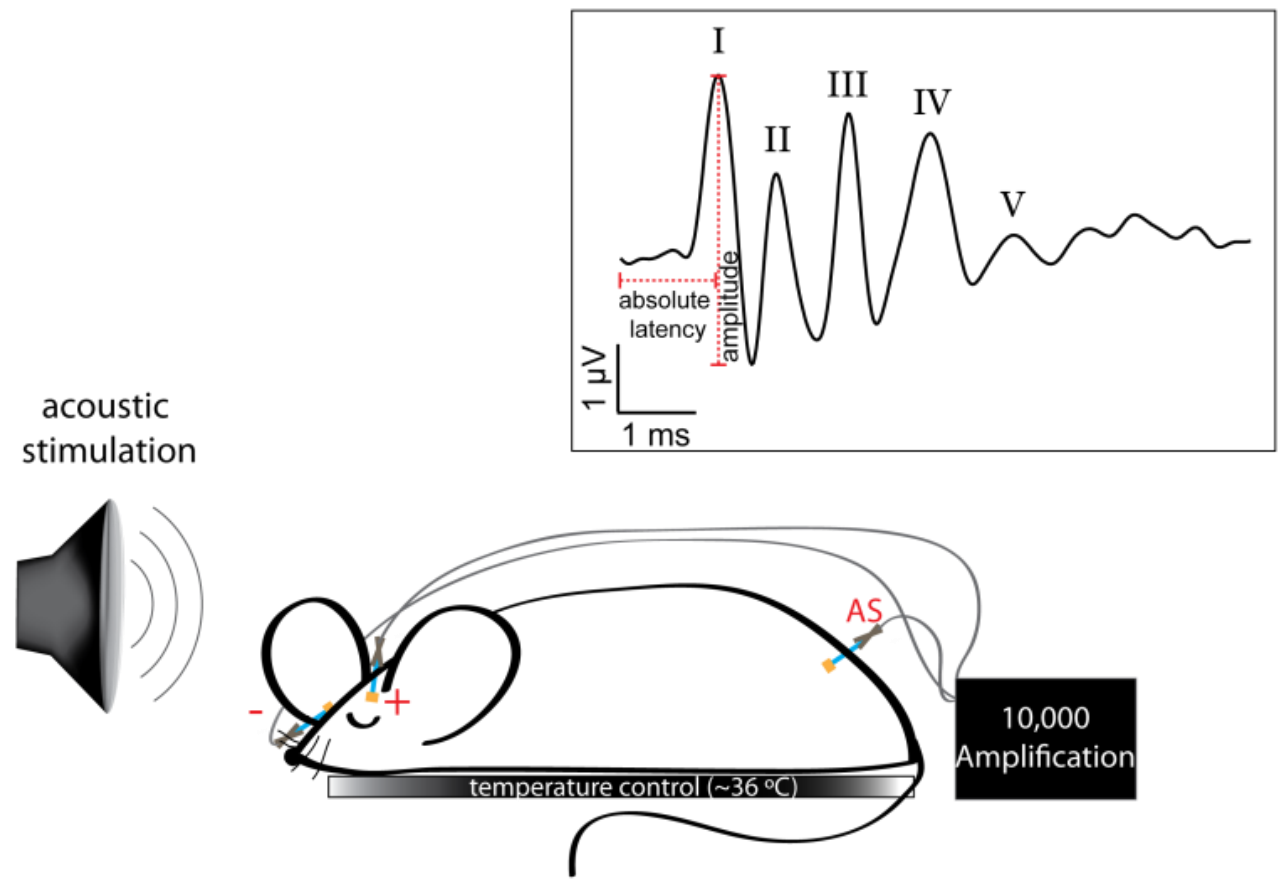

Figure 5. Schematic figure of the ABR recordings.

The mouse is anesthetized and placed over a heating pad to maintain its temperature $\left(\sim 36^{\circ} \mathrm{C}\right)$. Needle-tips are used as electrodes placed on the vertex (active, + ), the pinna (reference, -), and the back for active shielding (AS). After data amplification and processing, a series of 5 waves are obtained, which correspond to the response of different auditory stations. Peak-to-trough amplitude and absolute latency measurements were performed for each wave.

\section{II.VI.II. Specifics of experimental animals}

The numbers, genders and ages of mice used for ABRs for all the lines tested are summarized in the table below. Both males and females were pooled together for analysis.

Table 2. Summary of experimental animal numbers and characteristics used for auditory brainstem recordings.

\begin{tabular}{|c|c|c|c|c|c|}
\hline Mouse line & $\begin{array}{c}\text { \# controls } \\
\left(q / \delta^{\lambda}\right)\end{array}$ & $\begin{array}{c}\text { \# mutants } \\
\left(\Phi / O^{\lambda}\right)\end{array}$ & $\begin{array}{c}\text { Mean age } \\
\text { (weeks) / std }\end{array}$ & $\begin{array}{c}p \text { value } \\
\text { age }\end{array}$ & $\begin{array}{l}\text { p value speaker } \\
\text { distance }\end{array}$ \\
\hline $\mathrm{MBP}^{\text {ShII }}$ & $11(3 / 8)$ & $7(2 / 5)$ & $9.6 / 1.2$ & 0.09 & 0.3 \\
\hline $\mathrm{MBP}^{n e o}$ & $8(8 / 0)$ & $8(8 / 0)$ & $12.2 / 1.8$ & 0.5 & NA \\
\hline MCT1 & $9(5 / 4)$ & $7(4 / 3)$ & $11.9 / 1.8$ & 0.18 & 0.85 \\
\hline EMX1-MBP & $7(7 / 0)$ & $7(0 / 7)$ & $12.8 / 1.9$ & 0.65 & 0.13 \\
\hline
\end{tabular}




\section{II.VI.III. Analysis}

The voltage traces were bandpass filtered offline $(300-3000 \mathrm{~Hz})$ using a Butterworth filter of $6^{\text {th }}$ order. Data was divided from the stimulus presentation onset $(0 \mathrm{~ms})$ in $12 \mathrm{~ms}$ windows. Artifacts from the heart rate were removed (rejection threshold for the positive deflections was between 0.047 and $0.08 \mathrm{~V}$, and for the negative deflections between 0.08 and $0.092 \mathrm{~V}$ ). The mean across 1000 repetitions of each stimulus was used for the analysis. Hearing thresholds were determined by comparing evoked responses at each intensity with the residual background. The latter was calculated by taking the root mean square (rms) of the mean evoked responses over 1000 repetitions at $0 \mathrm{~dB}$ intensity. A window of $1 \mathrm{~ms}$ around peak I measured at $80 \mathrm{~dB}$ was used to calculate the maximal amplitude evoked at each intensity. The lowest sound intensity that elicited response amplitude 3 times larger than the residual background noise was considered the threshold for that animal. Visual inspection of the individual mean traces per animal was done, in order to confirm the sensibility of the threshold measure. The individual 5 waves were manually identified. Absolute latencies were determined by visually selecting the maximum peak value at $80 \mathrm{~dB}$ corresponding to each one of the 5 waves within the expected time window (approximately one wave every $1 \mathrm{~ms}$.). A template ABR was used for comparison and optimal differentiation of the 5 waves. Response amplitude was measured as peak-to-trough amplitudes.

\section{II.VII. Optic nerve recordings}

\section{II.VII.I. Solutions and electrodes}

Artificial cerebrospinal fluid (aCSF) was prepared, containing (in $\mathrm{mM}$ ): $124 \mathrm{NaCl}, 3 \mathrm{KCl}, 2$ $\mathrm{CaCl}_{2}, 2 \mathrm{MgSO}_{4}, 1.25 \mathrm{NaH}_{2} \mathrm{PO}_{4}$, and $23 \mathrm{NaHCO}_{3}$. Energy substrates were added for different protocols. $10 \mathrm{mM}$ glucose monohydrate (Fluka BioChemika, Munich Germany) was used for current/voltage dependence and conduction velocity measurements. $3.3 \mathrm{mM}$ glucose with 6.7 $\mathrm{mM}$ sucrose (Merck Millipore, Darmstadt, Germany) was used during high frequency stimulation. Glucose deprivation was done under $10 \mathrm{mM}$ sucrose. The before mentioned solutions were all adjusted to a $\mathrm{pH}$ of 7.4 and controlled for osmolarity. 4-aminopiridine (4-AP) $(\geq 98 \%$, Sigma) was used at different concentrations $(1,10,25,50,75$ or $100 \mu \mathrm{m})$ diluted in the previously mentioned $3.3 \mathrm{mM}$ glucose solution (working solution).

Both stimulating and recording suction electrodes were fabricated from borosilicate

glass capillaries (World Precision Instruments, Germany, 1B150-6, 1.5 mmx6", (152 mm) by 
heating the edge of one end, as described by (Stys et al., 1991). The capillary was bent smoothly in a $40^{\circ}$ angle and the heat generated a thickening of the glass at the opening, which became $\sim 0.3 \mathrm{~mm}$. A chloride silver wire (World Precision Instruments, Germany, 0.25 $\mathrm{mm}$ ) was inserted into the electrode, and the capillary space back-filled with aCSF with 10 $\mathrm{mM}$ glucose.

\section{II.VII.II. Optic nerve preparation and stimulation}

Mice between 8 to 14 weeks of age were euthanized by decapitation and the skull was exposed. The optic nerves (ON) were separated from the eyes at the ocular cavity. The skull was opened to gain access to the ventral side of the brain where both optic nerves were detached by cutting posterior to the optic chiasm. The preparation was placed into an interface brain/tissue slice (BTS) perfusion chamber (Harvard Apparatus, Holliston, MA) and continuously superfused with aCSF, bubbled with carbogen $\left(95 \% \mathrm{O}_{2}, 5 \% \mathrm{CO}_{2}\right)$ at $37^{\circ} \mathrm{C}$ during the experiment. The temperature was maintained constant using a feedback-driven temperature controller (model TC-10, NPI electronic) connected to a temperature probe (TS100-S, NPI electronic) inserted in the BTS incubation chamber. Both optic nerves were detached from the optic chiasm and they were individually placed into the suction electrodes for recording. The direction of the optic nerve was maintained constant throughout the experiments inserting the proximal (retinal) end of the nerve into the stimulation electrode as illustrated in Figure 6. The stimulating electrode was connected to a battery (Stimulus Isolator 385; WPI, Berlin, Germany) that delivered a supramaximal stimulus to the nerve. The recording electrode was connected to an EPC9/2 amplifier (Heka Elektronik, Lambrecht/Pfalz, Germany) for CAP recordings. Signals were typically amplified 500 times, low-pass filtered at $30 \mathrm{kHz}$ and acquired at $100 \mathrm{kHz}$. The reference channel was recorded by an aCSF-filled glass capillary next to the recording suction electrode, in contact with the bathing aCSF. Initial equilibration of the nerves was performed at $0.1 \mathrm{~Hz} / 0.75 \mathrm{~mA}$ stimulation, until the recorded compound action potential (CAP) showed a steady shape (typically between 10 to $40 \mathrm{~min}$.). To assess nerve excitability, the current/voltage dependence was measured in steps of increasing stimulation currents ( 0 to $1 \mathrm{~mA}$ in steps of $0.1 \mathrm{~mA}$ ). For ON recordings, 6 to 8 nerves from 3 to 7 animals (males and females) per group were used and pooled in the analysis. 


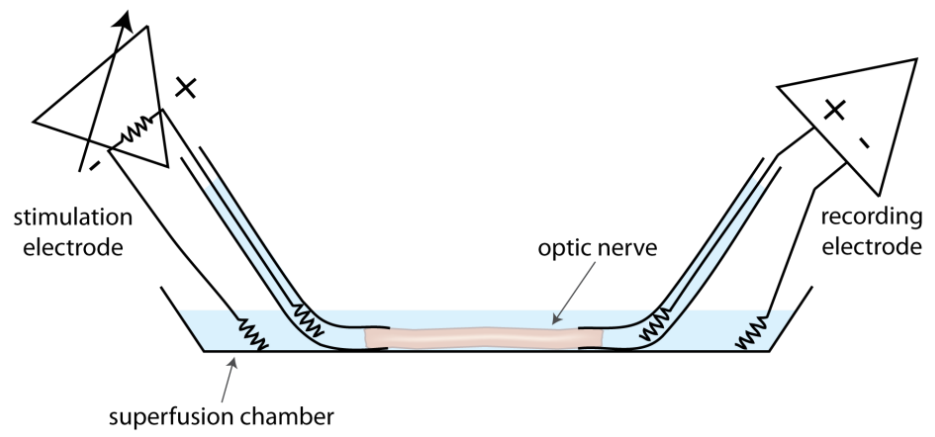

Figure 6. Schematic of the optic nerve recording setup.

The optic nerve is inserted into two suction electrodes (stimulation: left, recording: right) inside a superfusion chamber and maintained with constant supply of aCSF at $\sim 37^{\circ} \mathrm{C}$. Stimulation is always performed orthodromically. Scheme modified from (Saab et al., 2016; Trevisiol et al., 2017).

\section{II.VII.II.I. Current-voltage dependence}

To address the excitability of the ON, following optic nerve equilibration, an IV protocol was applied using currents intensities from 0 to $1 \mathrm{~mA}$ in steps of $0.1 \mathrm{~mA}$ with $10 \mathrm{~s}$ between each step. In some experiments, IV-dependence was measured before, and with 4-AP in the working solution, whose infusion started $20 \mathrm{~min}$. before.

\section{II.VII.II.II. Ramp stimulation}

To assess nerve responses to high-rate simulation (HRS), a ramp protocol was used. The protocol is a result of a series of 8 subsequent stimulations at 1, 4 (continuous), 7, 15, 25, 50 and 100 (burst-like) $\mathrm{Hz}$, for a total duration of $7.8 \mathrm{~min}$. Each burst-like stimulation consisted of 100 stimuli at the given frequency, separated by 460 ms during which the evoked CAP was recorded. The combination of 100 stimuli and CAP recording events were repeated to match a total duration of approximately $58.5 \mathrm{~s}$, independently from the rate. In some experiments, the ramp protocol was applied to nerves in the presence of 4-AP, whose infusion started $\sim 30 \mathrm{~min}$. before.

\section{II.VII.III. CAP analysis}

The CAP waveform of the $\mathrm{ON}$ is a measurement of axonal function and consists of 3 characteristic peaks with different amplitudes and latencies, each representing a different axonal population (see Figure 7). Given the absence of peak 1 in the mutant nerves, the 
analysis was performed independent of peak specification: either the maximal amplitude or the area under the curve were analyzed. The CAP area under the curve correlates with the CAP maximal amplitude and represents a reliable method to quantify the proportion of axons activated by the stimulation (Saab et al., 2016; Stys et al., 1991; Trevisiol et al., 2017).

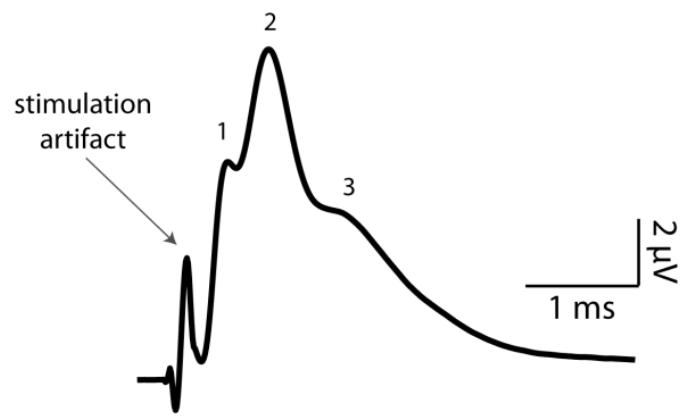

Figure 7. Compound action potential properties.

Classical optic nerve CAP shape consists of 3 peaks that reflect the activity of different caliber axons. The typical amplitude of the largest peak is $\sim 10 \mu \mathrm{V}$ when stimulating with $0.75 \mathrm{~mA}$.

\section{II.VII.III.I. CAP amplitude during IV curves}

To quantify nerve excitability, the CAP maximal amplitude was analyzed at each stimulation intensity. All amplitude values were normalized to those obtained at maximum stimulation intensity (1 mA), for comparison between groups.

\section{II.VII.III.II. Depolarizing and hyperpolarization area}

For the quantification of the differences in depolarizing and hyperpolarizing potentials, the CAP area was measured in a window of $15 \mathrm{~ms}$ starting at the end of the stimulus artifact. The area was obtained for the positive CAP values and taken as the depolarizing area, while the area of the negative CAP values was taken as the hyperpolarizing one.

\section{II.VII.III.III. High-rate stimulation}

It is known that HRS generates a reduction in the CAP amplitude (Trevisiol et al., 2017). For the analysis of nerve function during high-rate stimulation, the maximal CAP peak was analyzed at every time point of the ramp protocol. To obtain the relative decay in amplitude the data was normalized to the maximal CAP peak at the baseline condition before the ramp $(100 \%)$. 


\section{II.VII.III.IV. Conduction velocity}

The conduction velocity was obtained by dividing the length of the nerve by the CAP latency at its maximum amplitude value, elicited at $0.7 \mathrm{~mA}$. The conduction velocity values were normalized to the mean of control animals (100\%).

\section{II.VIII. Axon initial segment length quantification}

\section{II.VIII.I. Solutions and antibodies}

1) Cutting solution

Solution contained (in mM): $125 \mathrm{NaCl}, 25 \mathrm{NaHCO}_{3}, 1.25 \mathrm{NaH}_{2} \mathrm{PO}_{4} \cdot \mathrm{H}_{2} \mathrm{O}, 3 \mathrm{KCl}, 25$ glucose, 1 $\mathrm{CaCl}_{2}, 6 \mathrm{MgCl}_{2}, 1$ kynurenic acid.

2) 10x Phosphate buffered saline (PBS)

PBS contained: $1.7 \mathrm{M} \mathrm{NaCl}$, $34 \mathrm{mM} \mathrm{KCl}, 40 \mathrm{mM} \mathrm{Na} \mathrm{HPO}_{4} \cdot 2 \mathrm{H}_{2} \mathrm{O}, 18 \mathrm{mM} \mathrm{K}_{2} \mathrm{HPO}_{4}$ in distilled $\mathrm{H}_{2} \mathrm{O}$. With $1 \mathrm{~N} \mathrm{NaOH}, \mathrm{pH}$ was adjusted to 7.2 and solution was stored at room temperature. PBS $1 X$ was prepared from this solution by further diluting in distilled $\mathrm{H}_{2} \mathrm{O}$.

3) Blocking solution

$5 \%$ goat serum and $0.5 \%$ Triton $\mathrm{X}-100$ (10\% stock) in PBS.

4) Primary antibodies

Anti-K $\mathrm{K}_{\mathrm{v}} 7.3$ (KCNQ3n) raised in guinea pig (1:200) was kindly provided by Ed Cooper (Baylor College of Medicine, Houston, TX, USA) (Jin et al., 2009).

AnkyrinG antibody raised in rabbit (1:100; Santa Cruz).

5) Secondary antibodies

Alexa 488-conjugated donkey anti-rabbit (1:1000, Invitrogen).

Cy3 donkey anti-guinea pig, (1:1000, Dianova).

\section{II.VIII.II. Immunostaining}

The mouse was euthanized by cervical dislocation, the brain removed with surgical tools, submerged in ice-cold ( 15 min before procedure) cutting solution, and glued into a cutting plate. Coronal $300 \mu \mathrm{m}$ slices were prepared using a vibratome (VT1000S, Leica) with cutting amplitude of 1 and 0.05-0.07 forward speed. Freshly cut slices were fixed with Methanol chilled at $-20^{\circ} \mathrm{C}$ after removal of the cutting solution and incubated at $-20^{\circ} \mathrm{C}$ for $10 \mathrm{~min}$. Methanol was removed and the slices washed with PBS 1X (3 times, 5 min. each) dropped directly on top of the slices. Blocking lasted for 2.5-5 hours at room temperature. The primary antibodies dissolved in blocking solution were added and slices incubated for 2 days at room 
temperature. Slices where washed with PBS $1 \mathrm{X}$ (3 times, $5 \mathrm{~min}$. each), and secondary antibodies dissolved in blocking solution were added and left for 2 hours at room temperature (from the light). The slices were thoroughly washed with PBS $1 \mathrm{X}$ (4 times, $5 \mathrm{~min}$. each), stained with DAPI (4',6-diamidino-2-phenylindole) for 10min., and then washed with PBS 1X (4 times, 5min. each). Slices were mounted using Aqua-Poly/Mount (Polysciences, Inc) and stored at $4^{\circ} \mathrm{C}$ protected from the light. Immunostaining was done by Iva Tzvetanova with the help of Annete Fahrenholz.

\section{II.VIII.III. Imaging}

AIS were imaged using a Zeiss 510 meta confocal microscope. Z-stacks were done using a 40x objective ( $1 \mu \mathrm{m} z$ depth, $30 \mathrm{z}$ per stack in average). 5 fields were imaged per mouse. The imaging was done by Iva Tzvetanova.

\section{II.VIII.IV. Analysis}

AIS analysis was performed from confocal scans. AIS expression length of AnkyrinG and $\mathrm{K}_{\mathrm{v}} 7$ were measured with the segmented line tool in FIJI (v.1.51) by drawing a line along the AIS. For both channels the beginning and end of the expression was defined when the immunosignal was stronger than the background. No background subtraction was applied. Analysis was performed by Arne Battefeld.

\section{II.IX. Behavioral paradigms}

\section{II.IX.I. Pre-pulse inhibition of the startle reflex using silent gaps}

The acoustic startle response (ASR) is a transient motor reflex evoked by an unexpected loud sound (Figure $8, A$ and B). The ASR can be modulated either by internal or external factors. It is known that the presence of a non-startling pre-pulse or a silent gap in the background sound, 30 to $500 \mathrm{~ms}$ previous to the startle, can diminish the ASR generating a 'pre-pulse inibition' (Koch, 1999). Gap detection is a typical assessment to measure of temporal acuity, and it has been widely used in mammals (Allen et al., 2008; Anderson and Linden, 2016; Ison et al., 2002; Musiek et al., 2005; Ross et al., 2010; Valadbeigi et al., 2017; Walton et al., 2008). Gap-PPI can be efficiently used in mice to test temporal resolution (Popelář et al., 2017). The longer the gap previous to the startle sound, the more inhibition of the ASR it will elicit. 


\section{II.IX.I.I. Specifics of experimental animals}

A brief summary of the numbers, genders and ages of the experimental animal is provided in Table 3.

Table 3. Experimental animal numbers and characteristics used for PPI gap-detection

\begin{tabular}{|c|c|c|c|}
\hline Mouse line & $\begin{array}{c}\# \text { animals } \\
\left(\uparrow / O^{\lambda}\right)\end{array}$ & $\begin{array}{c}\text { Age } \\
\text { (weeks) mean/std }\end{array}$ & $p$ value age \\
\hline $\mathrm{MBP}^{+/ t}$ & $15(7 / 8)$ & $12.1 / 3.19$ & - \\
\hline $\mathrm{MBP}^{n e o}$ & $6(4 / 2)$ & 13.9/1.91 & 0.21 \\
\hline $\mathrm{MBP}^{+/=}$ & $6(4 / 2)$ & $11.71 / 0.46$ & 0.75 \\
\hline $\mathrm{MBP}^{e m x}$ & $6(0 / 6)$ & $10.6 / 0.74$ & 0.28 \\
\hline
\end{tabular}

\section{II.IX.I.II. Protocol and acquisition}

The experiment was performed inside a sound proof and anechoic room. During the procedure, the mouse was confined in a custom-made Plexiglas tunnel $(12 \mathrm{~cm}$ long by $4 \mathrm{~cm}$ diameter) (Figure 8). The 'startle tunnel' was in contact with a base that had a piezo element below (TRU components, 800 Ohms impedance, $50 \mathrm{~mm}$ diameter, spanning $30 \mathrm{~V}$ ). The piezo was connected to a data acquisition system (see section II.VI.I). Acquisition and sound delivery was done through a custom-made program in a Matlab environment. The data were acquired at a sampling rate of $1 \mathrm{kHz}$. The calibration of the testing apparatus was made using BBN sounds at different intensities and a Brüel \& Kjaer (4939 1/4" free field) microphone placed inside the startle tunnel, connected to a Brüel \& Kjaer amplifier (D4039, 2610, Denmark).

For testing, mice were acclimatized to the tunnel for 10 min before the start of the experiment. The acclimation time consisted of an initial period of 5 minutes of silence, followed by a second period of 5 minutes of background sound exposure. The background sound consisted of a broad band noise of $70 \mathrm{~dB}$ (SPL). The startle noise was a BBN pulse of $105 \mathrm{~dB}, 0 \mathrm{~ms}$ on/off ramp, and $40 \mathrm{~ms}$ duration. The session started with 10 startle-only pulses over the background sound at random times every 10-20 seconds to avoid sound prediction. This was followed by the gap-related trials, in which the background sound was interrupted with randomized presentations of different silent gap lengths $(0,1,2,3,5,7,10,25$ and 50 $\mathrm{ms}$ ). The startle sound always appeared $50 \mathrm{~ms}$ away from the end of the gap, irrespective of the gap length. In this phase, both gap and startle sounds had an on/off ramp of $1 \mathrm{~ms}$. In addition to the gap length randomization, the time between each trial was also randomized 
between 10-20 seconds. Each gap was presented 10 times. The experiment ended with five startle-only pulses, equal to the ones presented at the beginning, to assess habituation. The complete presentation of 105 trials had duration of approximately $30 \mathrm{~min}$.

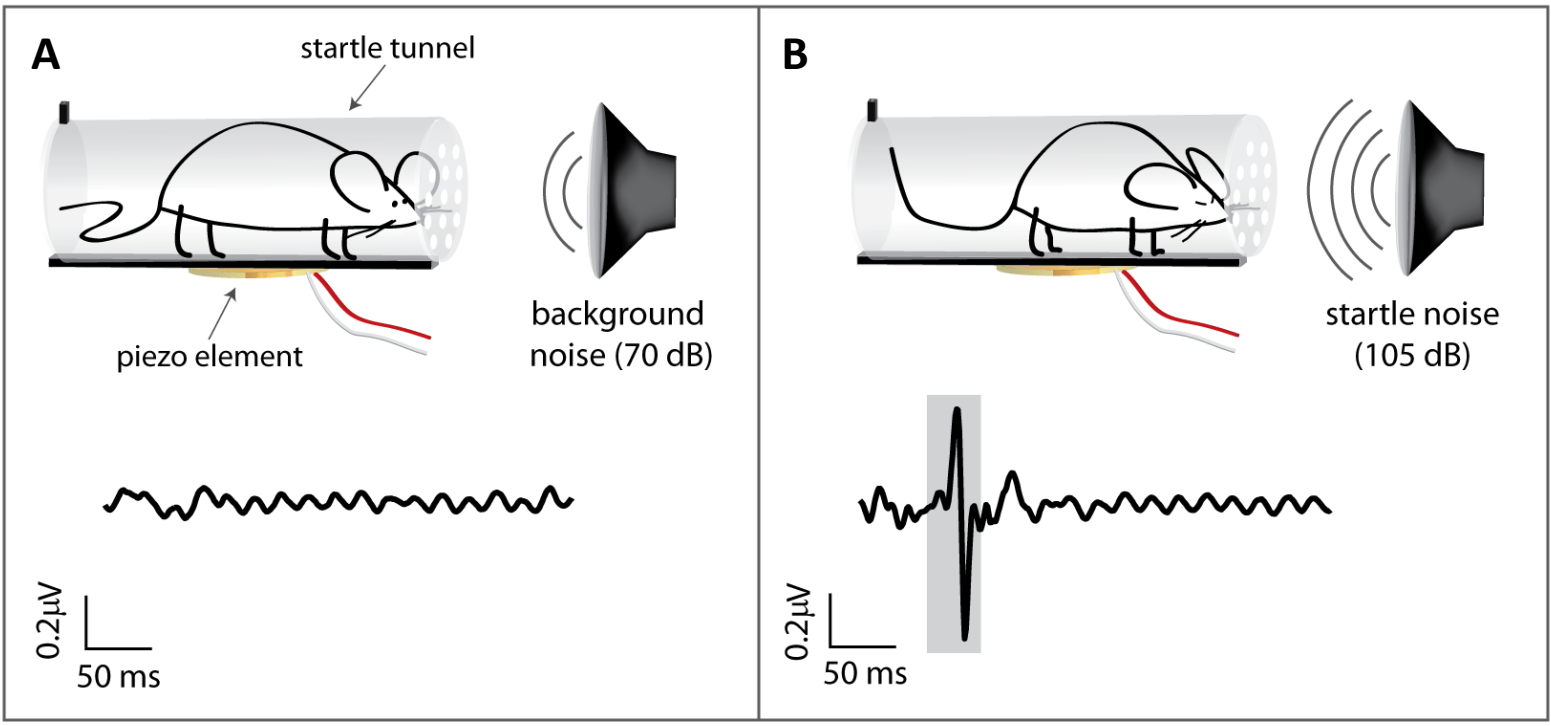

Figure 8. Gap-PPI testing paradigm.

A) A mouse is introduced in the startle tunnel for gap-PPI testing. A background noise of $70 \mathrm{~dB}$ plays and random presentations of the startle noise are introduced previous to a silent gap of different lengths. Recognizable gaps will elicit a reduction in the ASR. Only background activation of the piezo element is shown in the black trace. The voltage trace is very stable, because of minor movements of the animal. B) When an unexpected loud sound was presented, it caused a startle in the animal. The voltage trace detected by the piezo now shows a strong deflection in the potential, which correlates with the timing of the stimulus (shown by the gray shaded area).

\section{II.IX.I.III. Gap-PPI analysis}

Gap-PPI analysis was done as described before (Popelár et al., 2017), with mild modifications. The magnitude of the acoustic startle reflex (ASR) was measured as the maximal vertical force (peak-to-peak voltage output) exerted on the piezo element in a 500 $\mathrm{ms}$ window starting with the onset of the startle noise, minus the baseline activity (2 times the root mean square of the voltage trace in a $500 \mathrm{~ms}$ window before the startle noise). Noisy trials were discarded from the analysis, using a function of 3 times the standard deviation of the root mean square of the time before gap presentation for individual trials. Typically 1-5 trials out of a total of 105 were eliminated with this measure. The percentage of pre-pulse inhibition for each gap was calculated as in Equation 5.

\section{Equation 5}

$$
\operatorname{PPI}(\%)=100 \frac{A S R-A S R x}{A S R}
$$


Here, ASR is the startle response elicited at 0 ms gap (mean of 10 trials), and $A_{S} R_{x}$ is the startle response elicited after gap of length $x$. The curve of PPI elicited by different gap lengths was fit with a generalized logistic function:

Equation 6

$$
f=-\frac{a}{2}+\frac{a}{1+\exp (b+c * x)}
$$

I then obtained the gap detection threshold values. Recordings with a fit coefficient $\left(R^{2}\right)$ below 0.6 were excluded from the analysis. The gap detection threshold was the value of the fitted curve that elicited half of the maximal inhibition achieved per mouse. A second normalization was made to the longest gap $(50 \mathrm{~ms})$. Since no differences were observed between control animals of different lines, they were all pooled together.

\section{II.IX.II. AudioBox}

The AudioBox (NewBehavior, TSE systems) is an automated system for behavioral acoustic conditioning that allows for naturalistic testing (Brzózka et al., 2016; de Hoz and Nelken, 2014; de Hoz et al., 2017). This system is designed to minimize the interference from the experimenter and provides a spatially and socially enriched environment because the animals are housed in large groups while performing a task.

\section{II.IX.II.I. Behavioral system and animal procedure}

As seen in Figure 9, the setup consists of a home-cage, where animals have food ad-libitum and a sound box, which they can access through a corridor that ends in a corner. The corner contains two nose-poke holes connected to water bottles. Over the corner, there is a speaker (22TAF/G, Seas Prestige). The sound box is lined with sound-proof foam on the inside walls to avoid the generation of echoes.

For the mice to be recognized by the system, once they enter the corner, they were implanted with a transponder. Previous to exposure to the AudioBox, mice were anesthetized with Avertin mixture (250 mg/Kg body mass, i.p.) and injected with a 2.12 x $12 \mathrm{~mm}$ parylene coated transponder (ISO-Transponder FDX-B, Peddymark, Germany) under the skin on the 
neck. The tiny wound left by the injector was sealed with topical skin reabsorbible glue (Histroacry $\left.\right|^{\circledR}$, Braun, Germany). A period of 1-3 days in the home-cage with food and water ad libitum, was given for recovery from the implantation and to avoid a negative association to the AudioBox context. Mice were then placed in the AudioBox. For water access, animals had to go inside the sound box through a tunnel and enter the corner. Every time the animal entered the corner, its transponder was recognized by the system and a specific sound played for the duration of the corner visit. In this system, the animals learnt to associate specific sounds to the possibility of getting water or not. A typical paradigm consisted of two types of sounds: the 'safe', and the 'conditioned' sound. When a safe sound was played, the animal could make a nosepoke and get water. When a conditioned sound was played, the animal would get an air-puff when nose-poking, and the water doors would not open. This way, the mice learnt that certain sounds were conditioned and they should not try to nosepoke if they hear them. Once these safe and conditioned limits have been established and the animals learned, the testing of sounds that are similar to one or the other limit allowed the measurement of the perceptual capacities of the animals. 
A

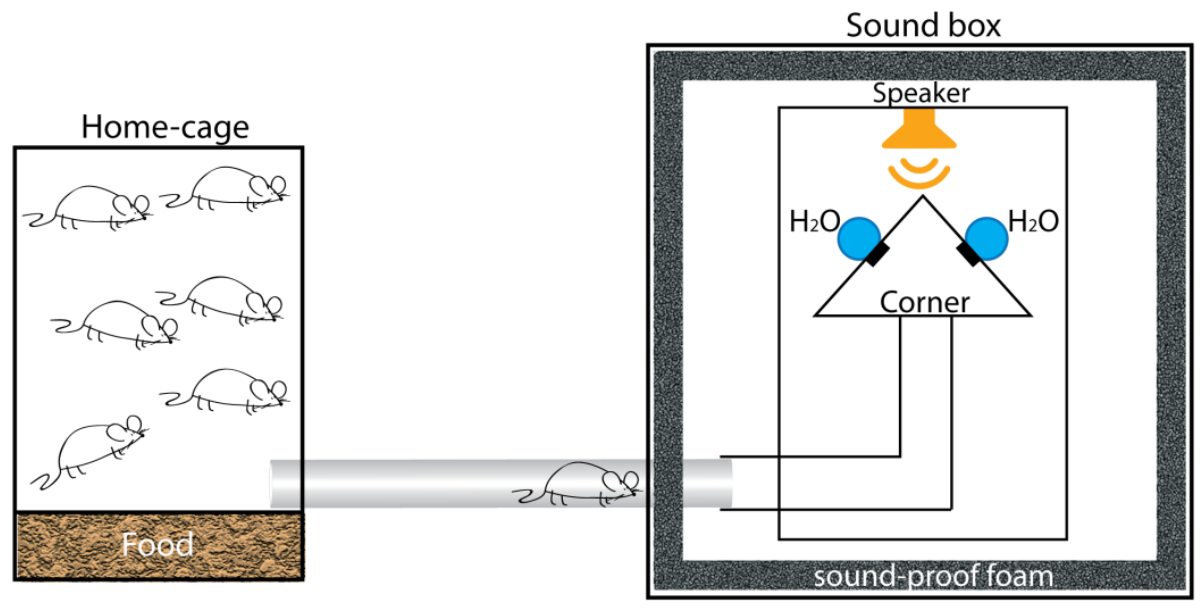

B

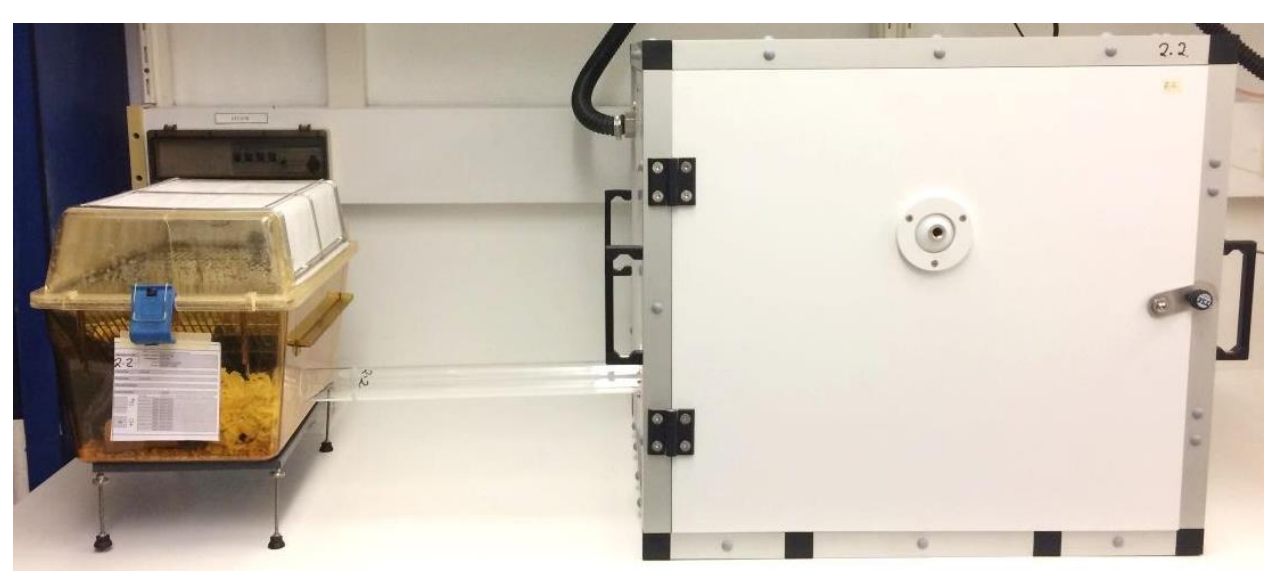

C

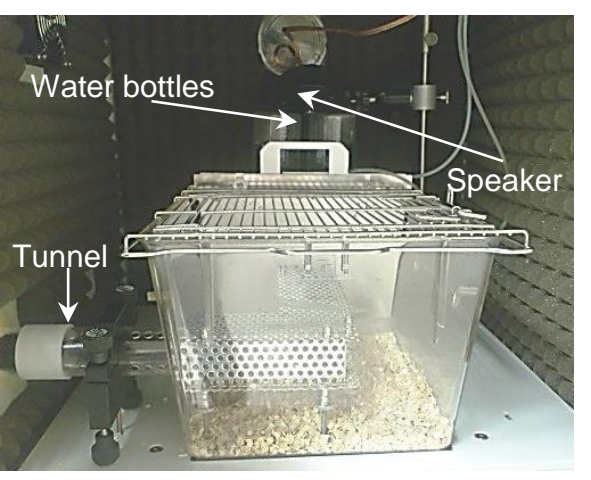

D

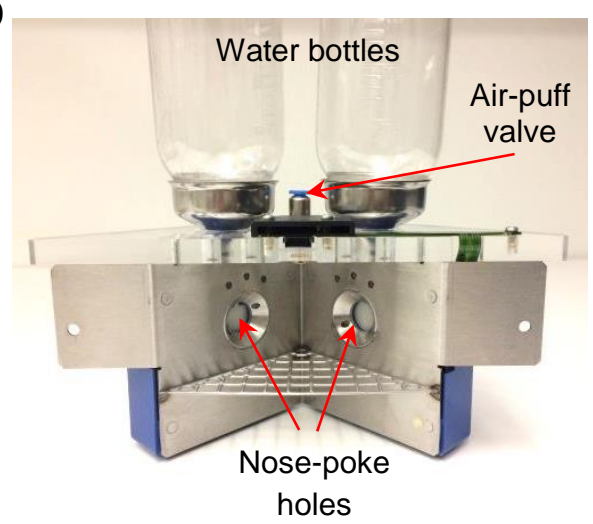

Figure 9. AudioBox setup.

The AudioBox is an automatic system for behavioral sensory testing. A) A diagram shows the composition of the setup. Mice lived in a home-cage with free access to food. To get water, they needed to enter the $C$ ) sound box and get into a D) corner, where they were recognized by the system. Once inside the corner, different sounds were presented. Animals need to learn the association between a sound and the possibility of drinking water. The number of visits with and without nosepokes was recorded and taken as a measure of the learning and sensory abilities of these animals. 


\section{II.IX.II.II. Gap detection paradigm}

I used the AudioBox system to test for behavioral gap-detection. In this case, the safe sound consisted of a continuous broad band noise that played for as long as the animal was in the corner. The conditioned sound was a broad band noise interrupted by $50 \mathrm{~ms}$ silent gaps (long, recognizable gaps) every 500 ms. The training paradigm, summarized in Figure 10, consisted of 3 phases. In the habituation phase (one day) the nose-poke doors were open and the safe sound was played on every visit. This phase allowed the animal to get acclimated to the environment. The animals learned that making a nose-poke gave direct access to water. The conditioning phase lasted 3 to 9 days, in which the water doors were closed, and the safe sound was played in all visits. In this period, the animals learned to make a nose-poke and wait for the doors to open to get access to water. During the conditioning phase the conditioned sound was introduced in a certain percentage of the visits. A low percentage of conditioning was used at the beginning to avoid strong negative associations to the corner. First there were $5 \%$ conditioned visits (this is $\sim 6$ visits per day, for a total mean of $\sim 140$ visits). After 2-4 days, the conditioned visits were progressively increased (every 2-4 days) to 10,15 and $20 \%$ of the total number of visits. Typically, after $1-2$ days in the $5 \%$ condition, the animals learned to discriminate both sounds. After the conditioning phase, the gap testing phase started, which consisted of the addition of two new sounds every four days with random gap lengths, while maintaining the safe and conditioned sound boundary. A total of 15 gaps were added between 1 and 45 ms of length.

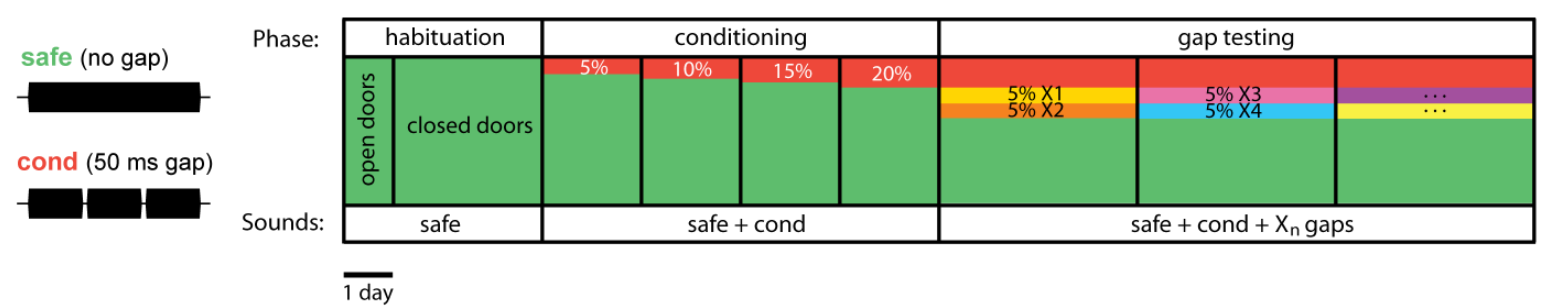

Figure 10. Summary of the AudioBox training protocol for gap-detection.

To test for behavioral gap-detection, mice were trained in the AudioBox to discriminate a "safe" sound (continuous broad band noise, green) from a conditioned sound (BBN with $50 \mathrm{~ms}$ silent gap embedded, red). The training paradigm is divided in 3 phases: habituation, conditioning and gap testing. In this timeline, horizontally is depicted the duration of each phase and vertically the percentage of exposure to specific sounds. Each phase is paired with the sounds that were presented accordingly. Habituation consisted in exposure to the safe sound only. During the conditioning, the exposure to the conditioned sound was progressively increased with days. Once $20 \%$ conditioning sound was reached, sounds with intermediate gaplengths were presented while maintaining the safe and conditioned boundary. 
The new presented sounds were chosen in pairs in a semi-random fashion, always taking care that the more difficult gaps (defined as all gaps below $5 \mathrm{~ms}$ ) were presented together with a gap longer than $5 \mathrm{~ms}$. Since the task becomes very difficult for smaller gaps, when testing for gaps $<3 \mathrm{~ms}$, the gap testing phase lasted for not more than two consecutive days. After two days, returning to $80 \%$ safe and $20 \%$ conditioned prevented the animals to have an aversive behavior to the task and to stop drinking water in further testing days. A minimum of four days in this phase was allowed before starting a new gap testing.

For this experiment, 3 replications were made and the total of animals, that showed effective learning were pooled together. In total, 7 control animals $\left(\mathrm{MBP}^{+/+}\right)$and 13 mutant animals $\left(\mathrm{MBP}^{\text {neo }}\right)$ that were 6-14 weeks old at the start of the experiment were used. $\mathrm{MBP}^{\text {neo }}$ mice were used, instead of $\mathrm{MBP}^{\text {shi }}$ because they have a normal life span and no motor impairments that could be the cause of reduced performance in the AudioBox.

\section{II.IX.II.III. Analysis}

Animals that did not nosepoke in more than $60 \%$ of safe visits during the conditioning phase were immediately excluded from the behavioral experiment and returned to a home-cage with free access to water and food. Other animals that during the testing protocol reached these levels were also excluded from the overall analysis. This was the case for 11 out of a total of $18 \mathrm{MBP}^{+/+}$and 7 out of $20 \mathrm{MBP}^{\text {neo }}$ tested.

For the behavioral analysis, the total amount of visits per day was averaged across the whole behavioral paradigm for all the sounds used for comparison of performance between groups. Also, the amount of visits per sound was compared between control and mutant animals to ensure the mice received equivalent sound exposure. The overall time spent in the corner depending on the sound that was played was quantified as a measure of perceptual ability. Also, the percentage of avoidance was quantified as the amount of visits without nosepokes for each sound played, only taking into account the first two days of training for each sounds. The statistical analysis was performed with an ANOVA for comparison between groups along all the gaps played that were $\geq 2 \mathrm{~ms}$. 


\section{II.X. Paw preference test}

\section{II.X.I. Test paradigm}

This test was developed by Collins (Collins, 1968) as a tool to assess the heritability of handedness in different mouse strains. We used a modified version of this test for the assessment of laterality indices in myelin mutant mice (Figure 11, A). Prior to the test, mice were food restricted (0.06-0.08 $\mathrm{g}$ food per gram of weight the night before). The test box consists of a transparent Plexiglas box that contains an open feeding tube ( $8 \mathrm{~mm}$ diameter) in the frontal wall. The dimensions of the box I used were: $10 \mathrm{~cm}$ wide $\times 10 \mathrm{~cm}$ deep, by $12 \mathrm{~cm}$ height, and the feeding tube was placed at $2.6 \mathrm{~cm}$ from the floor (Figure 11, B). These box dimensions allowed me to test myelin mutant animals with motor or anxiety disturbances.

The protocol consisted of two consecutive experimental days: a training and a testing day (Figure 11, C). The animals were manipulated and weighed every day for 3 days before the start of the experiment to reduce stress and habituate them to the experimenter. In addition, 10 food pellets (TSE dustless precision pellets, $14 \mathrm{mg}$ ) per day per animal were administered to avoid food neophobia. Access to water was ad libitum throughout.

On the training day, mice were introduced in the test box for 10 mins for habituation. Approximately 3-5 mins after the start of the habituation, a pellet was pushed inside the box through the tube, so the animal would understand the pellets are coming through the tube. Once the animal ate the pellet, a second pellet was placed on the inside edge of the tube, so the animal could access it with the mouth. Then, the session started, and individual food pellets were placed $\sim 0.5 \mathrm{~cm}$ away from the inside edge of the tube so that it could only be accessed with the paw. The video recording also started at this point (Sony Handycam DCRSR57 Carl Zeiss Vario Tessar, 25 ppm). The training session lasted either 20 min., or when the animal ate 25 pellets. All reaches with the right or left paw were recorded manually by the experimenter and video assessment was performed when visual inspection of the reaching movement was not achieved. Any attempt with both paws was considered as one ambidextrous attempt, and would be taken into account in the final count of the total paw entries (TPE). After the training session, $0.06 \mathrm{~g}$ of food per gram of the initial weight of the animal before starting the food restriction was administered together with 10 pellets. 
A
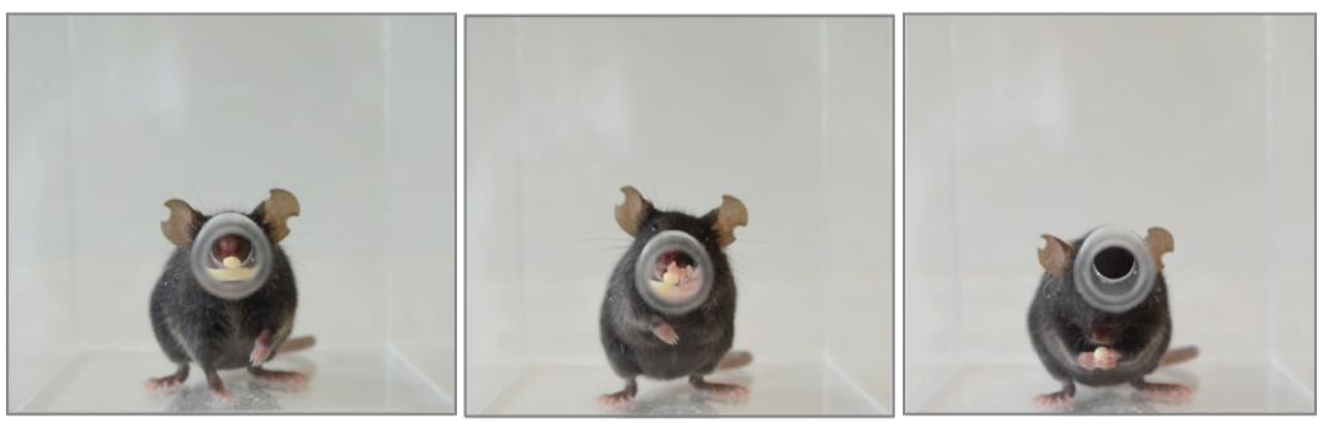

B

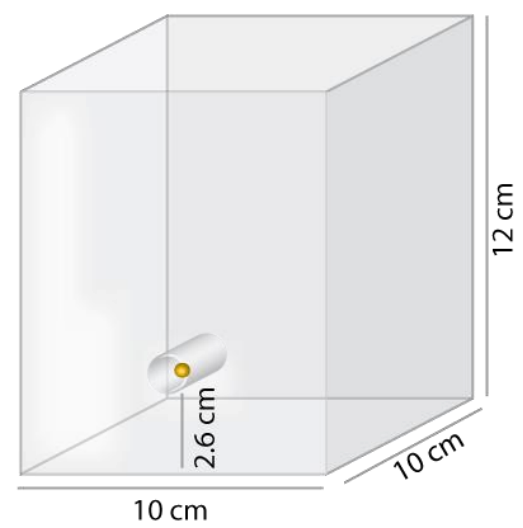

C

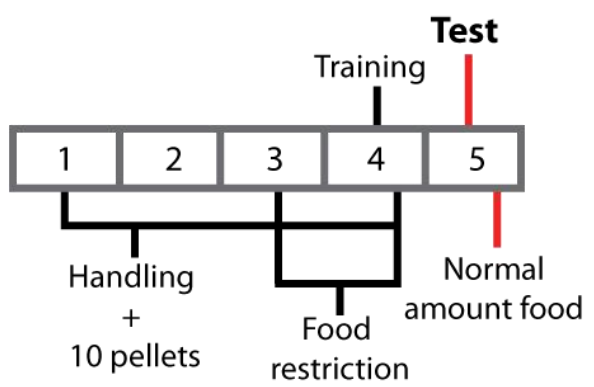

Figure 11. Paw preference protocol

A) Mice performing the paw preference task, from left to right is the time of decision making, reaching and taking the pellet. B) Adapted paw preference test box, with bigger dimensions. C) Paw preference protocol. Briefly, animals undergo a handling period of 3 days before training, in which they are also provided with 10 pellets per animal to get used to their flavor. A food restriction phase started $\sim 16 \mathrm{~h}$ before the training session. Animals received only $0.06-0.08 \mathrm{~g}$ of food per gram of weight overnight together with 10 pellets. After the training session, the same food restriction was applied over the original weight of the animal on the day right before the training. The test session, which was used for statistical analysis was very similar to the training session. After this, the normal amount of food was returned to the home-cage.

The test session took place the next day and was very similar to the training. The only difference was that the initial habituation period lasted for 3-5 min, and normal amount of food was administered at the end. The test session was the one considered for statistical analysis. In some cases, animals were tested consecutively, usually every 1 or 2 weeks. 


\section{II.X.II. Analysis}

From the amount of reaches the animals did during the test session, two different values were calculated: the directionality of lateralization (either right or left-handed) and the consistency (strong or weak, meaning very lateralized or ambidextrous respectively). To test of directionality, Equation 7 was used.

\section{Equation 7}

$$
\% R P E=\frac{R P E * 100}{T P E}
$$

Here, RPE was the total number of right paw entries an animal did during the session and TPE was the total amount of entries considering, right, left and ambidextrous attempts. This measure only takes into account the percentage of right versus left or ambidextrous entries. To test for strength of lateralization, Equation 8 was used.

\section{Equation 8}

$$
P P E i=\frac{\|R P E-L P E\|}{T P E} * 100
$$

Here, PPEi was the absolute value of the preferred paw entries, which would be independent of directionality. The strength of laterality was compared between groups using the absolute value of the PPE scored in the test session. For the statistical analysis of the directionality, the occurrence of right-pawedness was analyzed with a population distribution, by binning the animals' \%RPE in 10 bins from 0-100. In this way, the \%RPE was transformed into a frequency distribution, and analyzed with a chi square test. The confidence interval was $95 \%$. 


\section{Chapter 1}

\section{Not quite my tempo: auditory abnormalities caused by dysmyelination}

\subsection{Introduction}

\subsubsection{Hearing and sound}

An essential capacity for the survival of all organisms is the ability to sense their environment in an efficient and fast way in order to respond to diverse stimuli accordingly. One sensory system that relies on high temporal precision is the auditory system. The basic definition of sound, physically speaking, is that it is a pressure wave that propagates in the air. Natural listening environments are complex mixtures of sounds that the brain is able to separate into different objects. The sense of hearing is very complex, since it requires not only the physical detection of the wave properties, but an entire plethora of online short-term neuronal processes which include for example, assigning meaning to specific sounds, generating adaptation to background noise and even the capacity to respond when we are asleep. In addition, we are able to recognize certain objects by the sound they make, which allows us to differentiate sound sources and their relevance. For that reason, all the sounds in our environment carry valuable cues essential for survival. Hearing is, as defined by Phillips: 'the online perceptual elaboration of acoustic events distributed in time' (Phillips, 1999). Since the auditory pathway consists of a series of synaptic relays, the processing of sounds requires information arriving trough multi-synaptic pathways from the cochlea to the cortex. A precise 
timing of signals and processing of different information at different auditory stations must take place for the correct encoding of sounds.

\subsubsection{Temporal processing}

All sounds in our environment have a temporal structure, which affects the way we perceive and recognize them. In humans, identifying this temporal structure is essential for communication since it allows for speech perception (Frisina, 2001; Shen and Richards, 2013). In addition, it has an emotional importance as it plays a perceptual role in musical interpretation (Háden et al., 2015). Temporal processing refers to the neuronal ability of firing patterns that follow the temporal cues of sound (Musiek et al., 2005; Rosen, 1992). Spectral and temporal sound cues are represented as the carrier frequency and in the sound envelope respectively (Figure 12).

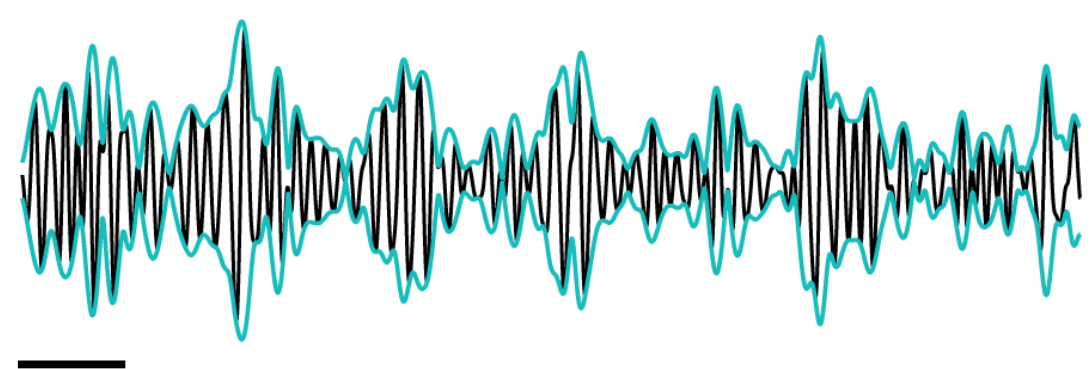

Figure 12. Temporal aspects of sound.

In a short trace of an amplitude modulated sinusoidal wave, we observe that carrier level (black trace, in this example happening at a frequency of $\sim 200 \mathrm{~Hz}$ ) has an amplitude modulated envelope (blue trace, in this example happening at a frequency of $\sim 30 \mathrm{~Hz}$ ) that confers the temporal dimension. Bar $=25 \mathrm{~ms}$.

Temporal perception in the auditory system has neuronal correlates that allow the encoding of temporal sound features. The capacity of auditory neurons to follow temporal cues relies on what is being transmitted into the brain trough the auditory nerve. In addition, the neuronal biophysical properties at different auditory stations might also play a role in information processing (Bayazitov et al., 2013; Nesse et al., 2010). Distinguishing the different temporal processes that the auditory system is able to perform, is important, since they might be coded by different circuits (Phillips, 1999).

The most studied temporal features are changes in sound envelope and the detection of onsets and offsets of sound. That is the reason why in this study, I focus on gap-detection and rate coding to understand how these aspects of auditory processing could be affected by myelin deficits. 
Temporal resolution is the ability of the auditory system to respond to rapid variations in the sound envelope over time. Whereas hearing problems related to frequency detection are mainly an auditory problem, failures in temporal processing in the auditory system might reflect a generalized neurological disorder (Eggermont, 2015). The highest level of temporal coding determination happens in the cortex (Eggermont and Wang, 2011) and several neurological disorders have been correlated with problems in auditory temporal processing, like schizophrenia, autism and epilepsy (Eggermont, 2015). In the next two sections, I will focus on the two main temporal processes studied in this thesis: rate coding and gapdetection.

\subsubsection{Rate coding}

When talking about the temporal structure of a sound, a difference between the carrier stimulus frequency (tone) and the stimulus envelope (contour) must be made (see Figure 12). Phase locking is the temporal representation of the auditory system to the period of a specific signal. Neurons can phase-lock to the envelope of the presented stimulus (Dreyer and Delgutte, 2006; Köppl, 1997). This is a characteristic of several sensory systems, and in the case of the auditory system, it varies greatly along the auditory pathway. It has been reported that the upper limit of rate coding decreases with the increase in auditory hierarchy (Joris et al., 2004). When presented with trains of pure tones, neurons in the auditory cortex usually can phase-lock approximately up to 15 hertz (Hz) (Creutzfeldt et al., 1980; Liu et al., 2006; Wehr and Metherate, 2011). In subcortical structures (e.g. the inferior colliculus) neurons can fire constantly throughout the stimulus and usually phase-lock up to several hundred $\mathrm{Hz}$. (Liu et al., 2006; Schnupp et al., 2015; Schreiner and Langner, 1988; Ter-Mikaelian et al., 2007) (see Figure 13).

This effect might be related to synaptic jitter, due to the multi-synaptic levels of processing that take place, but also due to differences in the biophysical properties of neurons at different auditory station (Liu et al., 2006). Typically, changes in periodicity following along the auditory system are often the result of adaptation, which is the reduction of response amplitude, and decreased temporal accuracy of the neurons when presented with continuous stimuli (Eggermont, 2015) 


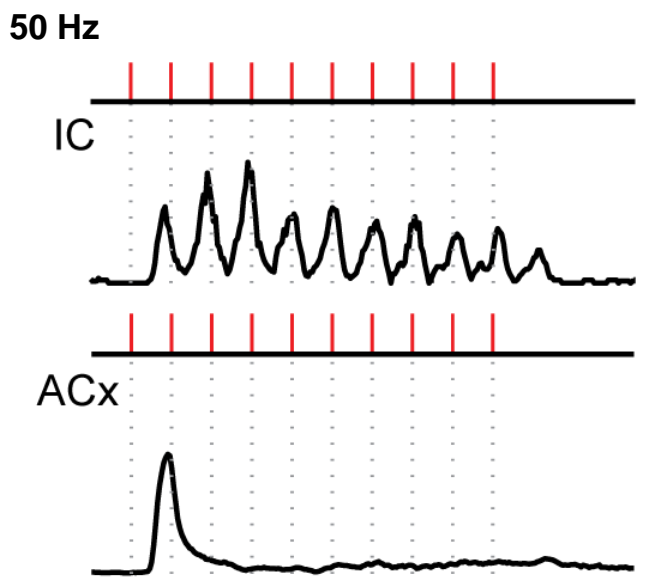

Figure 13. Examples of rate coding and spiking synchrony in different auditory stations.

A) Exemplifies the differences in rate coding between the inferior colliculus (upper) and the auditory cortex (lower). B) Shows the quantification of the strength of spike synchrony to the rate of the stimulus presented. As it has been extensively reported, here we can see that neurons in the IC have higher rate-coding abilities than neurons in the ACx.

The response of ACx neurons typically decreases with time, upon the presentation of repeated stimuli close in time (Wehr and Zador, 2005) and the effect is stronger when the inter-stimulus interval is shorter (see Figure 14). The 'adaptation' of these neurons is considered as a reversible mechanism that allows to contextualize firing patterns to current environmental stimuli (Scholes et al., 2011; Thompson and Spencer, 1966). This process has been termed 'forward suppression', which in the ACx, can last for hundreds of milliseconds and might be relevant for the coding of temporal patterns (Bayazitov et al., 2013). The specific mechanism responsible for the suppression of cortical responses is not yet known.

This suggests that cortical responses are dynamically adapted to the current auditory environment status (Bartlett and Wang, 2005), a process that is essential for context-specific auditory processing.
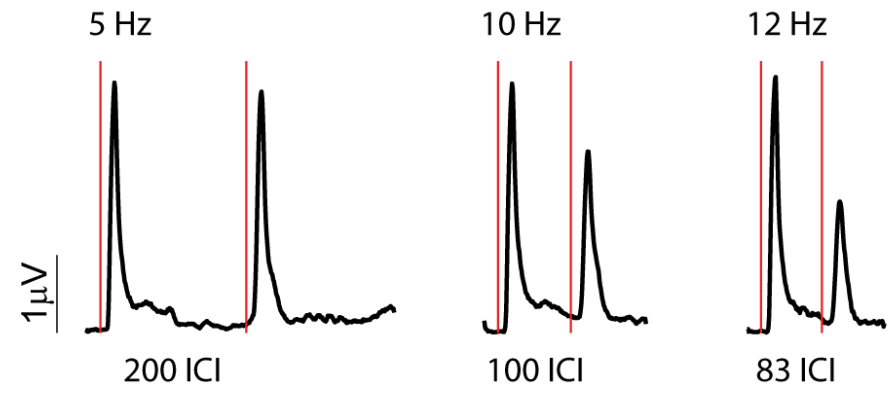

Figure 14. Example of forward suppression in the ACx.

Leftmost panel shows extracellular multiunit responses in the ACx of control mice to two consecutive click stimuli (depicted by vertical red lines) at $5 \mathrm{~Hz}(200 \mathrm{~ms}$ of inter-click interval). Since stimuli have enough separation in time, suppression is not seen in the second response compared to the first one. Middle panel shows the same responses as previously described, but for clicks presented at $10 \mathrm{~Hz}$ (100 ms inter-click interval). Approximately $25 \%$ of suppression is seen for this stimulus interval. Rightmost panel shows the responses to clicks presented at $12 \mathrm{~Hz}$ (83 ms inter-click interval). A $50 \%$ of suppression is seen in the second response compared to the first one. 


\subsubsection{Gap detection}

Temporal acuity is defined as the minimum perceptually detectable gap in a continuous sound (Irwin et al., 1981). Gap detection at the behavioral or electrophysiological level, is used to measure the sensibility or 'inertia' (Eddins and Green, 1995) of the auditory system to short variations in the temporal structure of the sound. This is one common method to measure temporal acuity (Phillips, 1999) used in clinical settings (Muluk et al., 2011; Shen and Richards, 2013) and in different animal models (Eggermont, 1999; Friedman et al., 2004; Hamann et al., 2004; Radziwon et al., 2009; Walton et al., 1997; Weible et al., 2014a). Temporal acuity comprises a fast process that requires de precise comparison of sounds within a short time period. It is believed that the study of the mechanisms underlying gap detection processes might help understanding speech perception and deficits related to speech discrimination (Phillips, 1999). Speech sounds consist of specific structures, usually periodic glottal pulses (carrier frequency of the voice) spaced by certain amount of time, which determines some of the voice features. The discrimination of phonemes, like /ba/ and $/ \mathrm{pa} /$, depends on the accurate detection of a gap (the voice-onset time), that occurs 20-40 ms between the release of a stop consonant (i.e. /b/) and the onset of voicing (Eggermont and Wang, 2011) (see figure Figure 15).
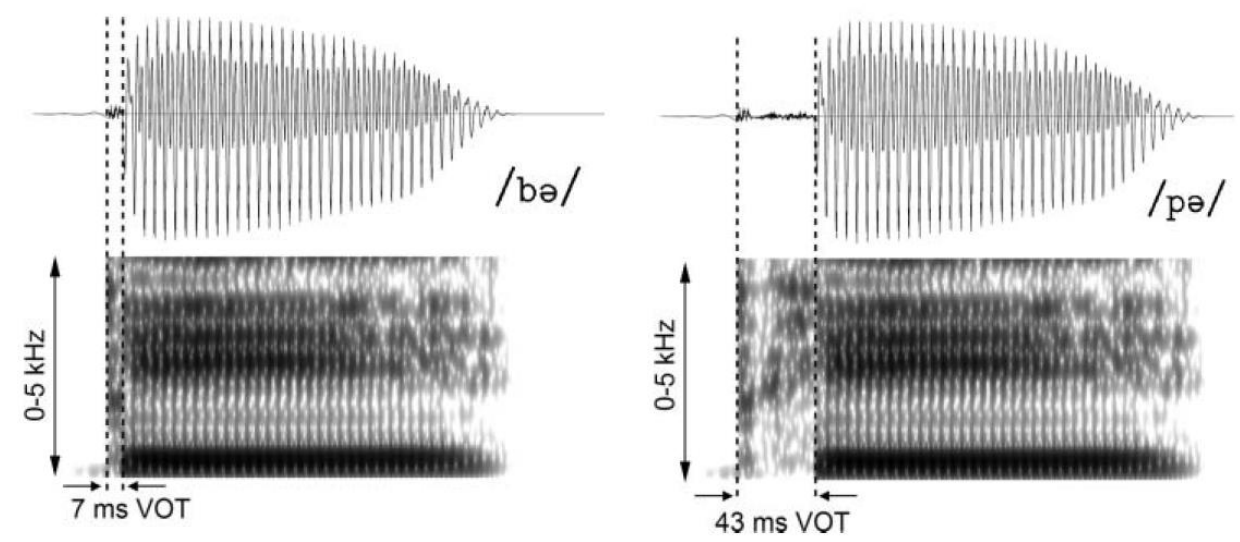

Figure 15. Exemplified voice onset time.

Synthetically generated /ba/ (left, $7 \mathrm{~ms}$ voice onset time) and /pa/ (right, $43 \mathrm{~ms}$ voice onset time) syllables. Upper plots show the waveforms and lower plots the spectrograms. Figure taken from (Neef et al., 2012). Copyright license ID: 4464670982374.

During ageing, gap-detection thresholds are increased despite corrections in the stimulus to avoid confounding effects of hearing loss (Eggermont, 2015); in this case, a correlation was found between gap-detection capacities and cognitive ability. In addition, gapdetection is impaired in patients with multiple sclerosis (Rappaport et al., 1994; Valadbeigi et al., 2017) an effect that was correlated by Valadbeigi, with a reduction in word discrimination. 
Children with reading and language impairments, whom also show higher gap-detection thresholds, might have difficulties in integrating fast-changing stimuli and overall temporal processing problems (Hautus et al., 2003), and it is proposed that gap-detection tasks should be used as screening tools in the early childhood to allow fast interventions and cognitive training for remediation (Merzenich et al., 1996). The study of temporal acuity through gapdetection is an optimal measure of the severity of changes in temporal processes, not only affecting the auditory system, but overall in the brain.

\subsubsection{Auditory disturbances related to myelin}

Since myelin regulates many processes to maintain the homeostasis of networks, and it plays an important role in regulating conduction velocity, it is expected that myelin abnormalities during pathological states would be reflected in impaired auditory function. Auditory neuropathies (ANP) are disorders related to deficiencies in the processing of sound temporal information (Zeng et al., 2005). ANP patients have normal cochlear function but deficits in peripheral (auditory nerve) and brainstem (Starr et al., 1996) regions, mainly due to deficient information transfer along the auditory nerve. ANPs have been related to myelin loss (Kim et al., 2013a; Long et al., 2018; Wan and Corfas, 2017). In contrast, patients with cochlear hearing loss tend to have impairments in thresholds or frequency processing but normal temporal processing (Eggermont, 2015).

Myelin defects could represent the underlying cause of certain auditory defects. Leukodystrophies, for example, are a series of pathologies associated to abnormalities in the CNS white matter, like hypomyelination. They are characterized by severe motor dysfunction, muscle rigidity and sensory abnormalities. There is a lot of heterogeneity in the literature regarding sensory loss in patients with leukodystrophies and hearing loss is sometimes not considered as a prominent sign of these pathologies (Leuzzi et al., 2000; Parikh et al., 2015). Nevertheless, reports show that patients present early hearing loss (Leuzzi et al., 2000). For example, in Palizaeus-Merzbacher Disease (PMD) patients, it is a common feature to find abnormal auditory brainstem responses (ABRs) which lack the activity of specific brainstem regions (Henneke et al., 2010; Kuan et al., 2008; Wang et al., 1995b). This could translate in a moderate hearing impairment. PMD is a hypomyelinating leukodystrophy associated with mutations in the PLP1 (the main protein component of CNS myelin). Interestingly, PMD-like patients (a form of the disease that has the same pathophysiology but without associated mutations in the PLP1 gene) have delayed but relatively normal ABRs (Henneke et al., 2010). 
In the case of multiple sclerosis (MS), the prevalence of auditory abnormalities in these patients is not entirely understood and it also appears to be very heterogeneous. Generally, auditory deficits are considered as a minor problem and it is also believed that due to the severity of the disease, MS patients do not complain about auditory symptoms. It has been reported that between $4-10 \%$ of MS patients develop sensory-neural hearing loss (Peyvandi et al., 2010). Several factors contribute to hearing problems in MS, including the state (either relapsing remitting MS or secondary progressive) (Lewis et al., 2010) and severity (Hendler et al., 1990). These variations make the clinical comparisons between patients and different techniques used for auditory testing, very difficult. The specific auditory abnormalities that MS patients present, pure tone hearing loss usually affects $50 \%$ of those who suffer from hearing deficits (Lewis et al., 2010). In addition, they have higher gapdetection thresholds and reduced speech-in-noise discrimination (Rappaport et al., 1994; Valadbeigi et al., 2014). Interestingly, from the percentage of MS patients with auditory deficits, in around $60 \%$ of the cases, the auditory deficit was the starting complaint that lead to the MS diagnosis (Hellmann et al., 2011). It has been suggested that MS lesions can be the cause of auditory temporal deficits and it is hypothesized that there is a certain variability of auditory abnormalities due to the area specific lesions disrupting different types of processing (Hendler et al., 1990). Finding auditory temporal abnormalities in MS patients indicates that these deficits belong to more central problems related to temporal processing than peripheral deficits related to frequency coding or changes in hearing thresholds.

A great variety of auditory deficits can be observed in patients with myelin-related diseases. This heterogeneity is due to the individual variability of myelin lesions and hearing profiles. Even though the pathophysiology might appear similar, the molecular causes of different disease types might be generating different sensory abnormalities, and this can be addressed by studying auditory function and understanding which hearing defects are related to which specific molecular changes. I strongly believe that auditory deficits in myelin-related diseases have been underestimated, and that understanding the role of myelin deficits in diverse aspects of auditory processing will help to pin point which are the major sensory deficits that arise when myelin is affected in specific ways. This, in the future, could also contribute to the understanding and development of more specific auditory tests to help the diagnosis and reveal the causes of different myelin related diseases. 


\subsection{Results}

\subsubsection{Absence of MBP results in a strong decrease of myelin in different auditory stations}

To understand the effect that the lack of myelin has in auditory processing, I tested the MBPshi mice that lack $~ 98 \%$ of the Myelin Basic Protein (MBP) (Lodygensky et al., 2012). MBP is a main component of CNS myelin (Boggs, 2006), localizes to the cytoplasmic areas of the myelin bilayer (Hildebrand et al., 1993) and it is essential for myelin compaction and major dense line formation (Privat et al., 1979). MBP $^{\text {shi }}$ mice constitute a well-known dysmyelination model, which has an autosomal recessive mutation on the MBP gene (Chernoff, 1981; Kimura et al., 1985; Kirschner and Ganser, 1980; Roach et al., 1983, 1985; Sidman et al., 1985). The mutation affects 5 out of the 6 protein-coding exons of the MBP (Roach et al., 1985) (see section I.VII). Since myelin is defined as the wrapped membrane present around axons, and the wrapping process depends partially on the compaction(Snaidero and Simons, 2017; Snaidero et al., 2014), MBP $^{\text {shi }}$ are basically a model animal without myelin in the CNS. At the cellular level, MBP ${ }^{\text {shi }}$ axons have only a few turns of non-compacted myelin (Kirschner and Ganser, 1980; Popko et al., 1987); in addition, they do not develop axonal dysfunction (Griffiths et al., 1998). Besides the strong dysmyelination phenotype, the oligodendrocytes appear normal (Kwiecien et al., 1998).

It was recently reported that myelin is quite heterogeneous in gray matter, not following the typical longitudinal distribution described before, and strikingly, showing intermittent myelin (Tomassy et al., 2014). Since it would appear from this, that myelin in the cortex is serving another role of that of regulating conduction velocity, I was interested in studying gray matter auditory areas and the role that myelin can have in sensory processing. My two main auditory areas of study were the auditory cortex (ACx) and the inferior colliculus (IC) (see Materials and Methods, section II.V.I). White matter tracts, such as the optic nerve (Brady et al., 1999; Kirkpatrick et al., 2001) corpus callosum (Dyakin et al., 2010) or spinal cord (Inoue et al., 1981; Privat et al., 1979; Rosenbluth, 1980; Windrem et al., 2008), have been widely characterized in the $\mathrm{MBP}^{\text {shi }}$ mice regarding myelin content. Nevertheless, it was important to understand how the myelination patterns in my areas of study would be in MBP ${ }^{\text {shi }}$ animals. 
The first step was to characterize the gray matter of $\mathrm{MBP}^{\text {shi }}$ mice and to achieve this, I collaborated with the Electron Microscopy (EM) facility of the Max Planck Institute of Experimental Medicine. Dr. Wiebke Möbius and Torben Ruhwedel did EM of MBP ${ }^{\text {shi }}$ mice from the ACx and IC. We can observe a brief scheme of the auditory pathway (as represented in Figure 1), where the areas of interest are highlighted in yellow (Figure 16-A). Both samples from the ACx and IC were obtained and processed by Torben Ruhwedel. As expected, MBP shi axons show a strong dysmyelinated profile, both in the IC and in the ACx (Figure $16 \mathrm{~B}$ ). Interestingly, myelinated axons are seen more often in the IC than in the ACx of control animals, but are low in number in both compared to a typical white matter tract. By zooming into the $\mathrm{ACx}$ axons, we can clearly see that control animals have compact (electrodense) myelin, while $\mathrm{MBP}^{\text {shi }}$ show profiles of failed myelination, often with several oligodendrocyte processes trying to wrap but not successfully closing on the axons. The quantification of the myelinating profiles in both groups shows a strong absence of wrapped axons in $\mathrm{MBP}^{\text {shi }}$ mice (Figure 16-C).

\subsubsection{Functional sensory consequences of the total MBP loss in grey and white matter}

Once knowing the distribution of myelin at the relevant auditory areas I am studying, I wanted to address the functional consequences of the reduction of MBP at the level of the auditory system. To assess basic auditory processing, I measured auditory brainstem responses (ABRs) in $\mathrm{MBP}^{\text {shi }}$ and $\mathrm{MBP}^{+/+}$mice. ABRs are very small auditory potentials evoked by sound, measured in vivo from the skull of the mice. Once amplified, filtered and processed, the response of different auditory brainstem stations can be characterized by the presence of specific waves. ABR potentials usually consist of 5 waves, each one belonging to the activity of a certain auditory station. 
A

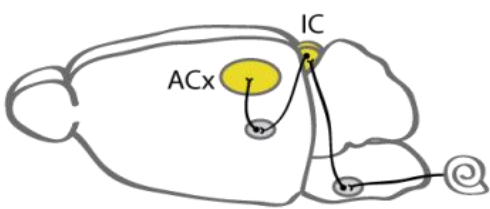

B

$\mathrm{MBP}^{+/+} \quad \mathrm{MBP}^{\text {shi }}$

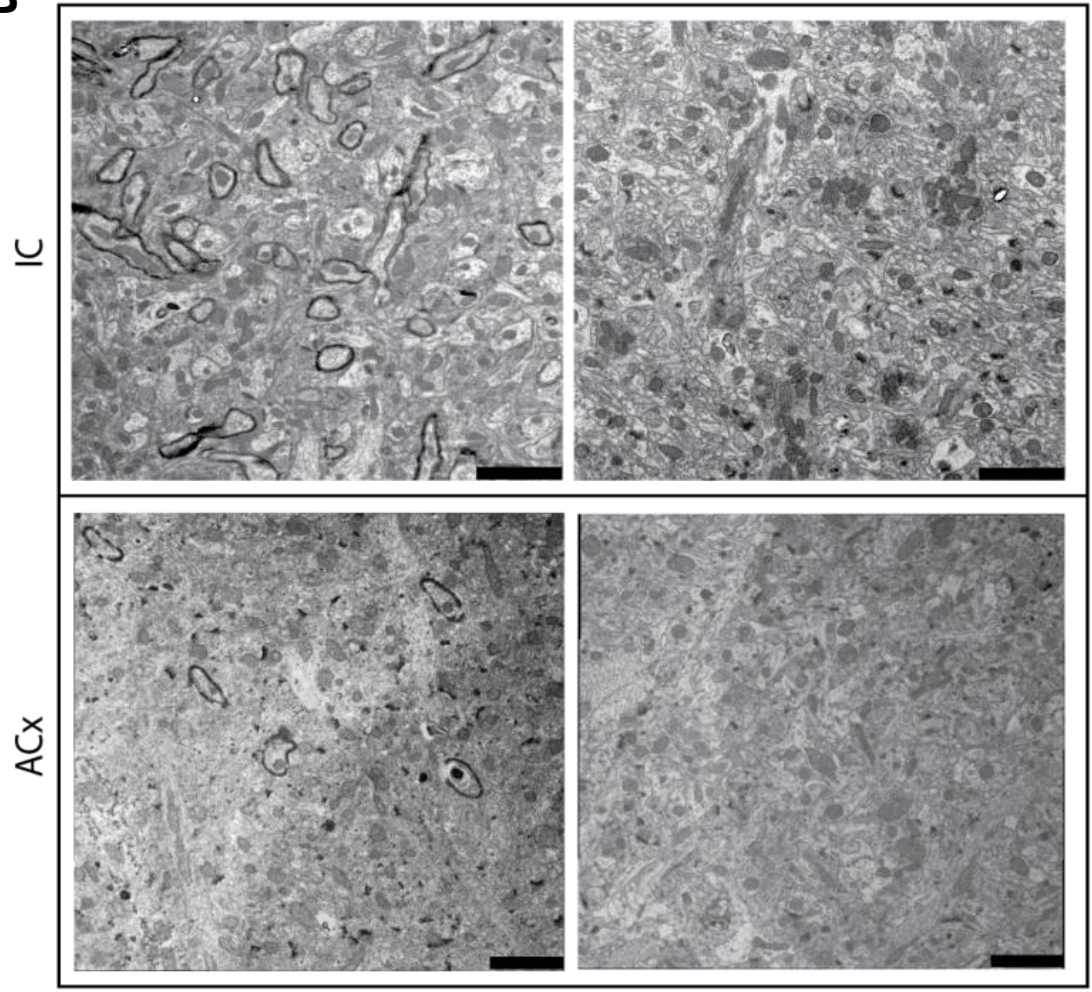

C
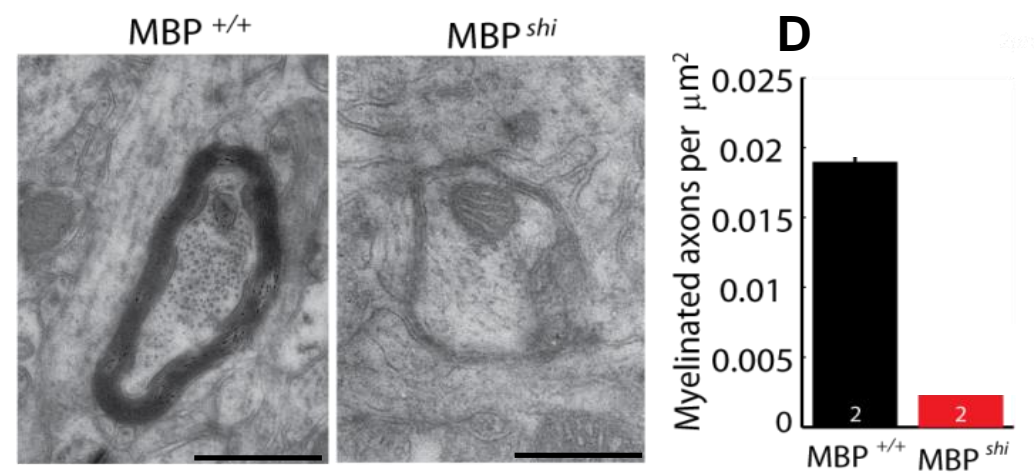

Figure 16. Myelin profiles in the inferior colliculus and the auditory cortex of $\mathrm{MBP}^{s h t}$ mice.

A) Schematic diagram showing the auditory pathway; highlighted in yellow the areas of interest. B) Example of myelination profiles in the inferior colliculus for control (left) and $\mathrm{MBP}^{\text {shi }}$ mice (right). Bottom figures show the same profiles located in the ACx. In these examples, the extent of myelination is higher in the IC than in the $A C x$, and in both cases, $\mathrm{MBP}^{\text {shi }}$ mice present no axons with compact myelin. Bar size $=2 \mu \mathrm{m}$. C) Zooms of a control and an MBP ${ }^{\text {shi }}$ axon is shown from the ACx. MBP ${ }^{\text {shi }}$ axons show oligodendrocytes trying to wrap axons without successfully forming compact myelin. D) These profiles were quantified for 20 areas in control and 20 areas for mutant animals (2 mice per group). Quantification shows the overall mean of the 20 areas per group and the S.E.M. Barely no axons with myelinated profiles are seen in mutant animals. Images in B and $\mathrm{C}$ were kindly provided by Torben Ruhwedel. 
The following table depicts the correlation of wave number and auditory station:

Table 4. Correlation between ABR wave and auditory station activity.

\begin{tabular}{cc} 
Wave & Station \\
\hline I & Auditory nerve \\
\hline II & Cochlear nucleus \\
\hline III & Superior olive \\
IV & Lateral lemniscus \\
V & Inferior colliculus \\
\hline
\end{tabular}

\section{Basic hearing properties}

Since wave I reflects the activity of the auditory nerve, and the nerve consists of a more peripheral section myelinated by Schwann cells and another part (more central) myelinated by oligodendrocytes (Wan and Corfas, 2017; Xing et al., 2012), it was important to distinguish if the measured effects in the mutant animals were abnormalities related to processing at the CNS level, or came from changes in the activation of hair cells in the cochlea, or latency differences due to defects in myelinating cells from the periphery. I recorded ABRs in MBP ${ }^{\text {shi }}$ and control mice. In this experiment, control animals were a combination of $\mathrm{MBP}^{+/+}$and $\mathrm{MBP}^{+/-}$ mice, since I did not observe any differences between $\mathrm{MBP}^{+/+}$and $\mathrm{MBP}^{+/}$mice at the $\mathrm{ABR}$ level. A summary of the measurements applied is shown in Table 5. In addition, no differences were seen in between groups in the threshold of hearing $(p=0.54)$. For this reason, I decided to pool $7 \mathrm{MBP}^{+/+}$and $4 \mathrm{MBP}^{+/}$mice for analysis to be treated as the control group, only in the case of the shiverer mice.

Table 5. Comparison of amplitude and intensity ABR measurements between $\mathrm{MBP}^{+/+}$and $\mathrm{MBP}^{+/-} \mathrm{mice}^{\text {. }}$ The reported numbers for amplitude and latency measurements from each wave correspond to the $p$ values extracted from non-parametric rank sum tests between $\mathrm{MBP}^{+/+}$and $\mathrm{MPB}^{+/}$mice, showing no differences between groups.

\begin{tabular}{ccc} 
Wave & Amplitude & Latency \\
I & 0.65 & 0.67 \\
\hline II & 0.53 & 0.78 \\
III & 0.41 & 0.15 \\
IV & 0.41 & 0.11 \\
V & 0.96 & 0.13
\end{tabular}


As observed in Figure 17-A, MBP ${ }^{\text {shi }}$ mice have several changes in the auditory brainstem potentials elicited by a click sound of $80 \mathrm{~dB}$. We can see that wave II seems split in two and merged with wave III. Threshold of responses to click stimuli presented from 0 to $80 \mathrm{~dB}$ showed that, despite the differences in waveform, there are no changes in threshold $(p=0.54)$ between control and $\mathrm{MBP}^{\text {shi }}$ mice (Figure 17-B). Latencies of $\mathrm{MBP}^{\text {shi }}$ to all waves are significantly increased $(p<0.001, p<0.0001, p<0.0001 p<0.0001, p<0.0001$ respectively for all 5 waves) (Figure 17-C). MBP $^{\text {shi }}$ have a significant reduction in the response amplitude of waves II and III, and a significant increase in the amplitude of waves IV and V $(p=0.44$, $\mathrm{p}<0.0001, \mathrm{p}=0.0031, \mathrm{p}=0.022, \mathrm{p}=0.008$ for waves $\mathrm{I}$ to $\mathrm{V}$ respectively) (Figure 17-D). Since the increase of wave IV and V amplitude was quite striking, I measured the ratio between peripheral and central areas as reported before (Schaette and McAlpine, 2011; Sergeyenko et al., 2013) to understand the relative increase of central auditory gain. The typical wave used for this quantification is wave $\mathrm{V}$, nevertheless, wave $\mathrm{V}$ is usually the most variable and the hardest to detect. Since I observed a significant increase in amplitude of wave IV, as well as wave V, I quantified the relative ratio for both waves, together with wave III for comparison purposes To understand the relationship between peripheral and central processing and the relative increase in central gain, caused by the lack of myelin, an analysis that comprises the relationship between wave I amplitude and central waves amplitude was done as reported before (Schaette and McAlpine, 2011; Sergeyenko et al., 2013), for wave III, IV and V. In Figure $17 \mathrm{E}-\mathrm{G}$, we can observe no significant differences for the ratio of wave $\mathrm{III} / \mathrm{I}(\mathrm{p}=0.085)$, but I saw a significant increase in the wave IV/I $(\mathrm{p}=0.026)$ and wave $\mathrm{V} / \mathrm{I}(\mathrm{p}=0.006)$ in $\mathrm{MBP}^{\text {shi }}$ mice. These results mean that at the latter auditory brainstem station (i.e. lateral lemniscus and inferior colliculus) there is a gain increase in $\mathrm{MBP}^{\text {shi }}$ mice, probably as a compensatory mechanism for the loss of wave II-III stability. The gain increase also seems to be elevated between lateral lemniscus (wave IV, gain of $\sim 44 \%$ ) and the inferior colliculus (wave V, gain of $\sim 230 \%)$. 
A

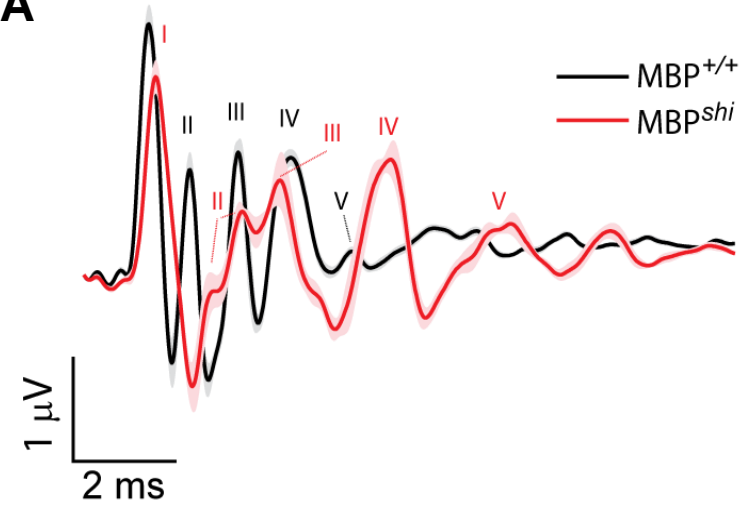

B

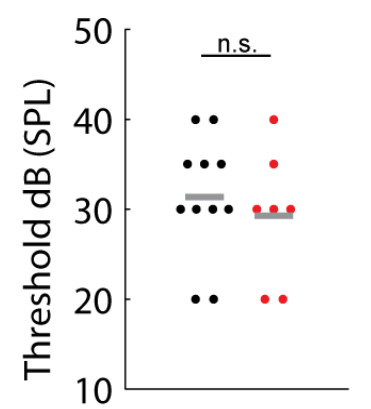

C

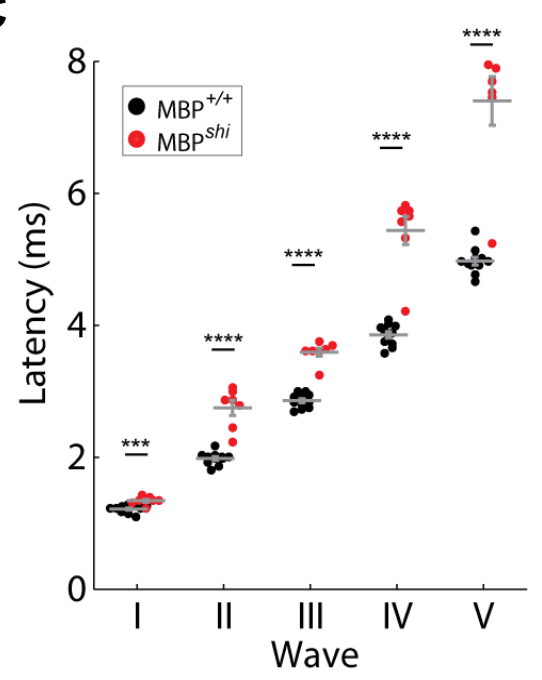

D

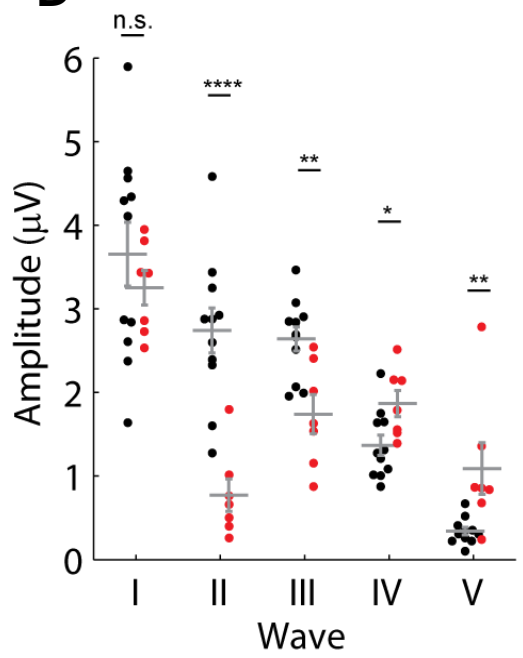

E

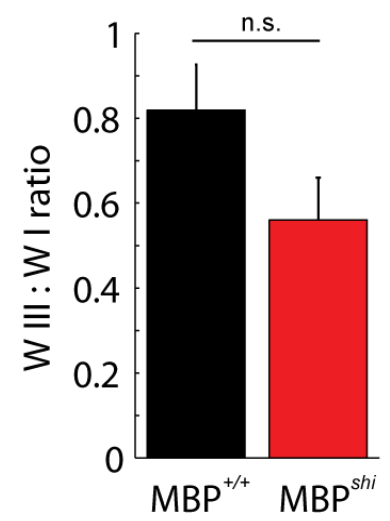

$\mathbf{F}$

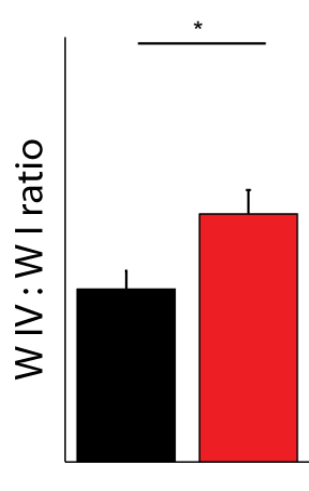

G

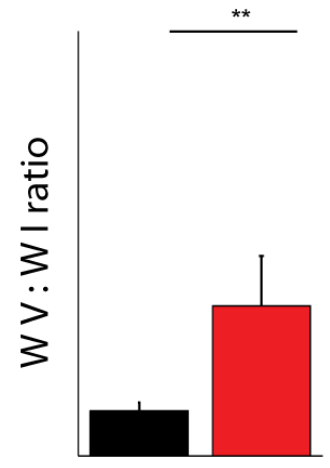

Figure 17. Subcortical auditory processing is impaired in $\mathrm{MBP}^{\text {sht }}$ mice.

A) The 5 characteristic ABR waves belonging to 5 different auditory stations, from the auditory nerve (I) until the inferior colliculus (V) for control (black) and mutant (red) animals. MBP ${ }^{\text {shi }}$ mice (red), show strong delays in auditory potentials elicited by clicks at $80 \mathrm{~dB}$. In addition, shape of waves II and III are merged. B) No differences are seen in auditory thresholds ( $p=0.54)$, and indirect measure of outer hair cell function. C) A significant increase in all 5 waves is seen in MBP $^{\text {shi }}$ animals, compared to control ( $\mathrm{p}<0.001$ for all waves). D) MBP ${ }^{\text {shi }}$ mice have a reduction in response amplitude to waves II and III ( $p<0.0001$ and $p=0.0031$ respectively), while waves IV and $\mathrm{V}$ are enhanced $(\mathrm{p}=0.022, \mathrm{p}=0.008)$. Central auditory gain quantification as the ratio between wave III, IV and V compared to I. E) No changes in central auditory gain were observed for wave III ( $p=0.085)$. A significant increase in central auditory gain was seen for $F$ ) wave IV $(p=0.026)$ and $G)$ wave $V(p=0.006)$. All plots show the mean + S.E.M. $n=11$ control and $n=7$ mutant animals. 
I next confirmed the expected increase in latency in the evoked-multiunit activity (MUA) in higher order areas. By recording the extracellular activity of a small population of neurons, a more specific readout of the changes in neuronal firing is achieved. I did MUA recordings from the IC (the last auditory station measured with the ABRs) and compared it with the ACx. In Figure 18 we can observe the responses of $\mathrm{MBP}^{+/+}$and $\mathrm{MBP}^{\text {shi }}$ mice to a click stimulus. It is evident the strong responses delay in $\mathrm{MBP}^{\text {shi }}$ mice both in the $\mathrm{ACx}$ and at the IC (Figure 18-A and D respectively). A significant increase in latencies is seen in both structures for the $\mathrm{MBP}^{\text {shi }}$ mice $(\mathrm{ACx} \mathrm{p}<0.0001, \mathrm{IC} \mathrm{p}=0.014)$ (Figure 18-B and $\mathrm{E}$ respectively).
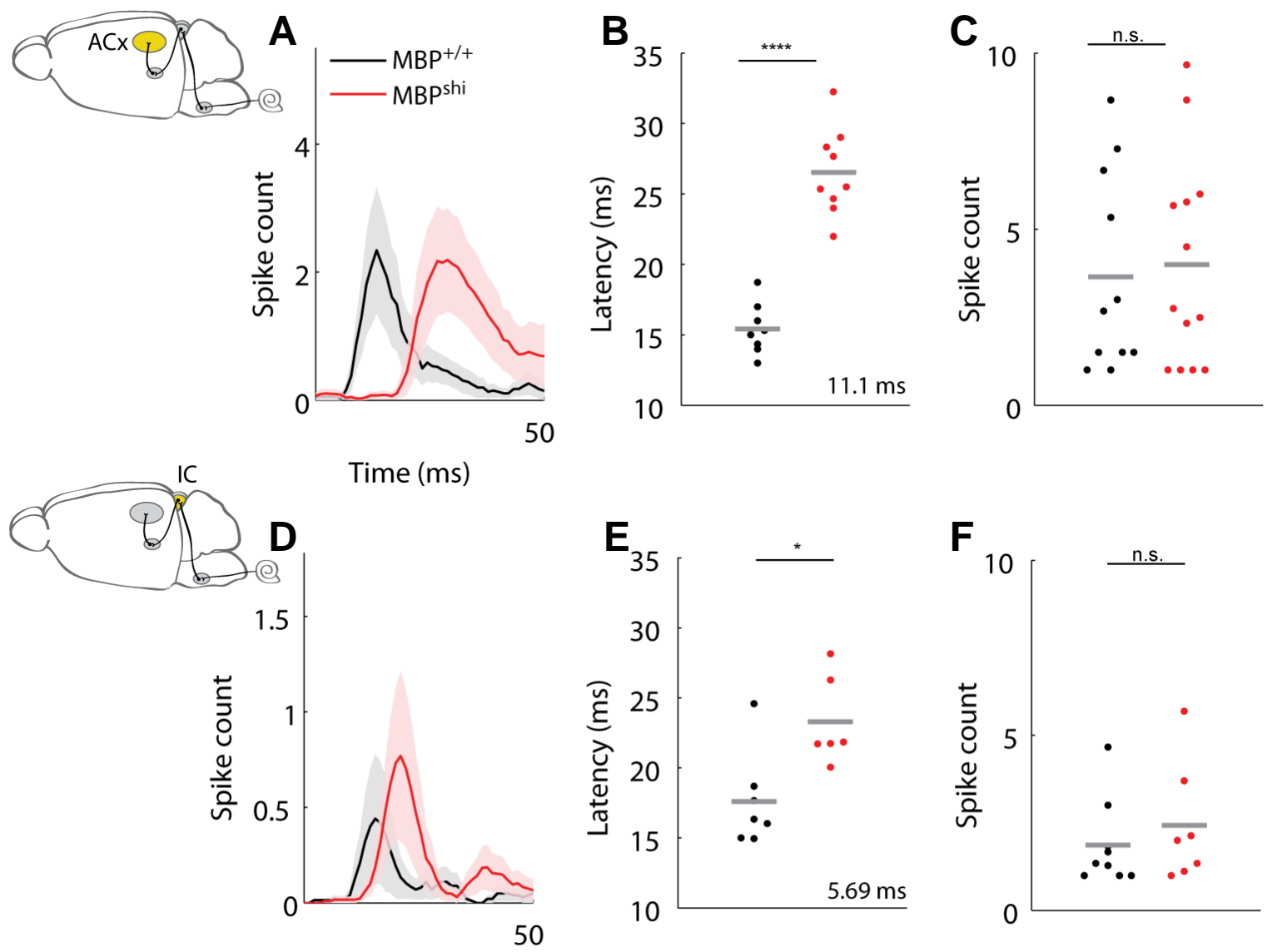

Time (ms)

Figure 18. Auditory cortex and inferior colliculus responses are impaired in MBP ${ }^{s h i}$ mice.

A) PSTH of the auditory cortex response to a click stimulus for control (black) and MBP ${ }^{\text {shi }}$ (red) mice. A strong increase in response latency is seen at this level. A) A strong latency increase is seen in mutant (red dots) animals $(p<0.0001)$ compared to controls (black dots); number in the right lower corner depicts the difference between control and mutant means. Grey bars depict the mean per group. C) No significant differences are seen in response amplitude in the $A C x(p=0.97)$. D) Same as in $A)$ but for the inferior colliculus. Latency shift is not so strong in the inferior colliculus compared to the cortex. E) Response latency is significantly increased in the IC of MBP $^{\text {shi }}$ mice $(p=0.014)$, nevertheless, the differences between the means is almost half of what is seen in the ACx (5.96 ms vs $11.1 \mathrm{~ms})$. F) No significant differences between groups are seen in response amplitude in the IC $(p=0.39)$. ACx $n=9-14$ mutant mice and $n=7-11$ control mice. IC $n=6-7$ mutant mice and $n=7-8$ control mice. 
Nevertheless, there is no change in the response amplitude in neither structure $(A C x p=0.97$, IC $p=0.39$ ), suggesting that these areas are not affected by conduction blocks.

I was also interested in understanding if there was a change in the width of tuning curves, since it would be expected that naked dysmyelinated axons could cross-activate each other (via ephaptic activations) and hence this could result in a broadening of the tuning curves in $\mathrm{MBP}^{\text {shi }}$ mice. To test this, I recorded ACx responses to pure tones at different intensity levels (see Materials and Methods section II.V.V.I). I observed no differences between the tuning widths in $\mathrm{MBP}^{\text {shi }}$ compared to controls (Figure 19). In addition, I did not observe differences in the best frequency of the recorded areas at $80 \mathrm{~dB}(p=0.57)$, suggesting that recordings in both control and mutant animals were done in homologous regions and that dysmyelination does not affect frequency tuning.

A
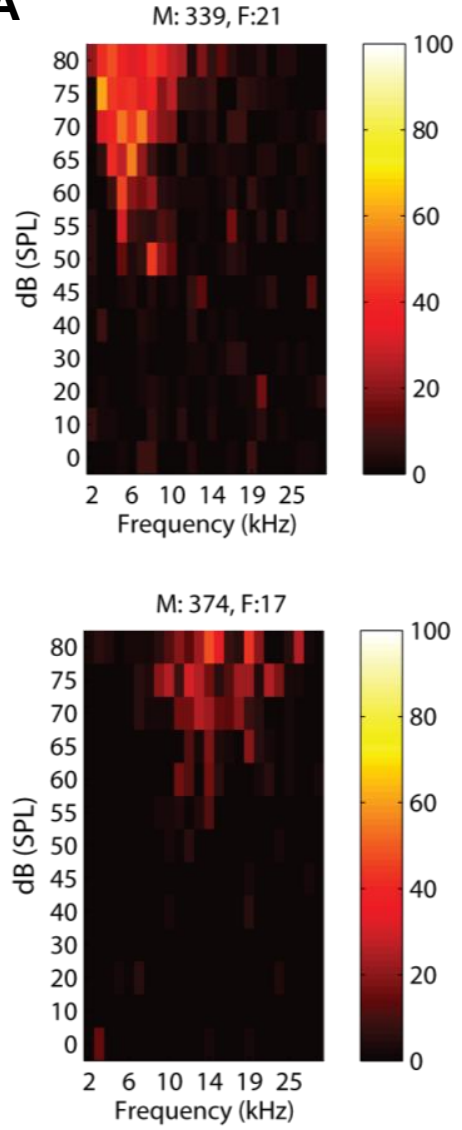

B
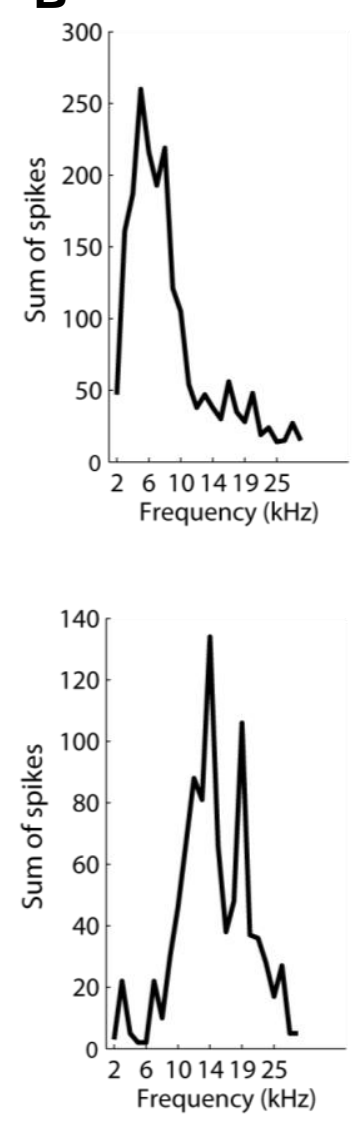

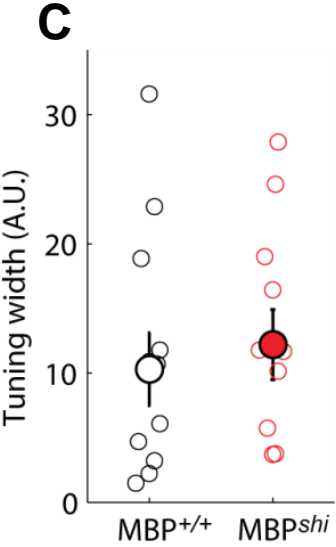

Figure 19. Frequency tuning is normal in $\mathrm{MBP}^{\text {shi }}$ mice.

A) Heat map plot shows an individual example of a control (upper plot) and a mutant (lower plot) mouse. Recordings in the $\mathrm{ACx}$ aimed to assess frequency response areas. ACX neurons were stimulated with pure tones at different intensities and frequencies. B) From the spike count along frequencies and intensities, the tuning curves were constructed for control (upper) and mutant (lower) animals. C) The spread of the TC was measured to asses tuning width. No differences were seen between groups $(p=0.63) . n=11$ control and $n=10$ mutants. 


\section{What is happening in the cortex?}

I was puzzled by the fact that it seemed that inputs from subcortical areas were overamplified, probably to compensate for the lack of synchrony at the initial stations of the auditory system, but nonetheless, responses in the ACx appeared normal in amplitude. It has been widely reported that the site of action potential initiation, the axon initial segment (AIS) undergoes plastic remodeling upon the absence of stimuli or with hyperexcitable environments. In this way, it allows the regulation of neuronal excitability by extending or shortening the area of ion entry, or getting closer or farther away from the soma (Evans et al., 2015; Grubb and Burrone, 2010; Gutzmann et al., 2014; Kuba et al., 2014; Yamada and Kuba, 2016). In an effort to understand if the microenvironment of cortical neurons in MBP ${ }^{\text {shi }}$ mice showed signs of over-stimulation and how dysmyelination was affecting AIS structure, I collaborated with Dr. Arne Battefeld and Prof. Dr. Maarten Kole, to do axon initial segment stainings from $\mathrm{MBP}^{\text {shi }}$ mice. With the help of Iva Tzvetanova and Annette Fahrenholz, we assessed the length of the AIS in MBP ${ }^{\text {shi }}$ mice. AnkG is one of the major scaffolding proteins of the AIS, and it is commonly used to measure AIS length (Grubb and Burrone, 2010; Gutzmann et al., 2014; Hamada and Kole, 2015; Jones and Svitkina, 2016; Kole and Stuart, 2012; Kuba et al., 2014; Yamada and Kuba, 2016). All AIS proteins are directly or indirectly anchored to AnkG (Jones and Svitkina, 2016; Leterrier et al., 2017; Rasband, 2010). In this case, we used AnkG and $K_{v} 7.3$ as a measure of AIS length. AnkG and $K_{v} 7.3$ proteins were immunostained in layer II/III or cortical neurons of control and $\mathrm{MBP}^{\text {shi }}$ mice. In Figure 20 we can observe example figures of auditory cortex layer II/III AIS staining for AnkG and $\mathrm{K}_{\mathrm{v}} 7.3$ in controls (Figure 20-A) and $\mathrm{MBP}^{\text {shi }}$ mice (Figure 20-B). We found a strong reduction in AnkG length in $\mathrm{MBP}^{\text {shi }}$ cortical axons $(\mathrm{p}=0.035)$ (Figure 20-A), while the span of the $\mathrm{K}_{\mathrm{v}} 7.3$ channels was unchanged $(p=0.57)$ (Figure $20-B)$. This suggests that dysmyelination is generating AIS remodeling, probably to modulate the excitability of naked axons trough a shortening of the AIS. 

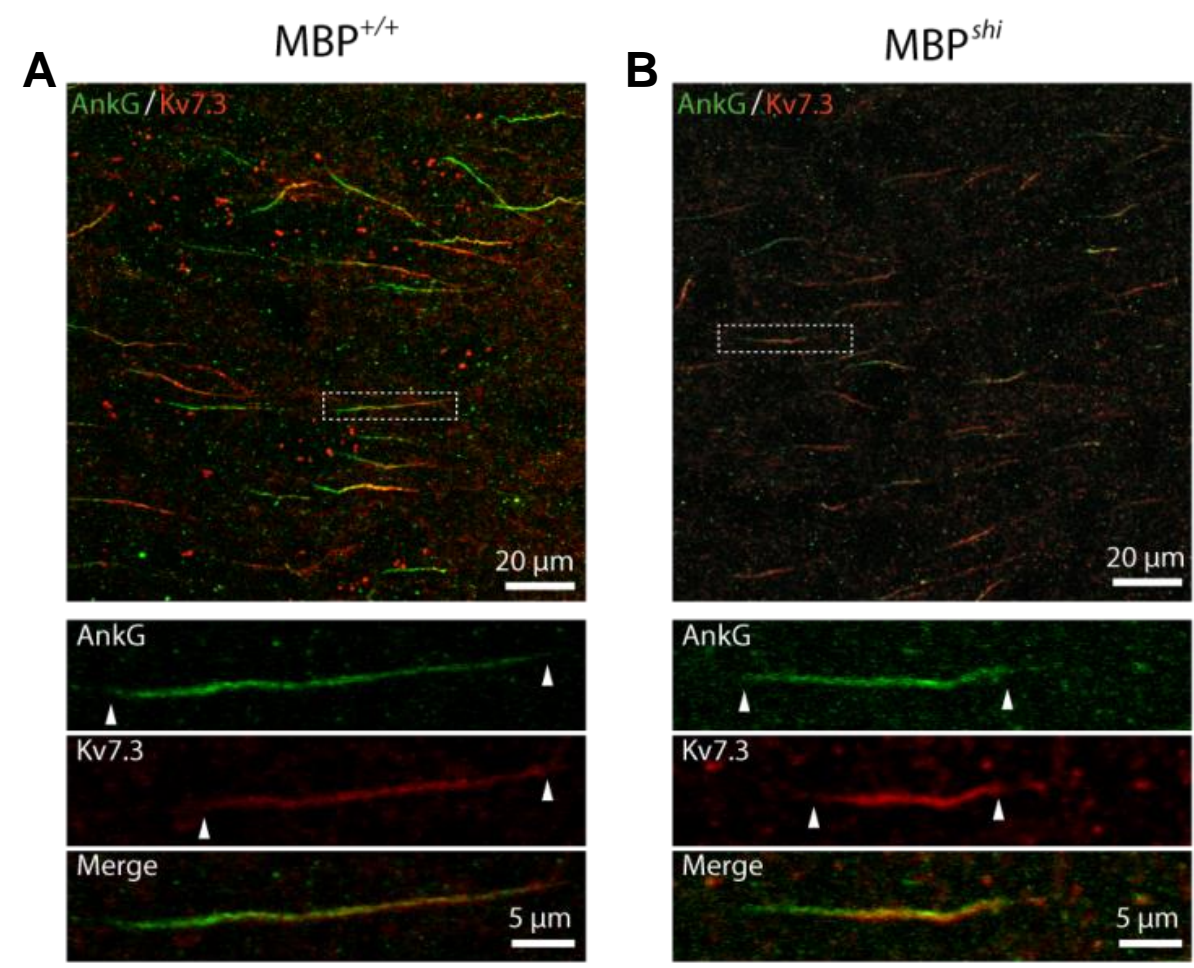

\section{C}
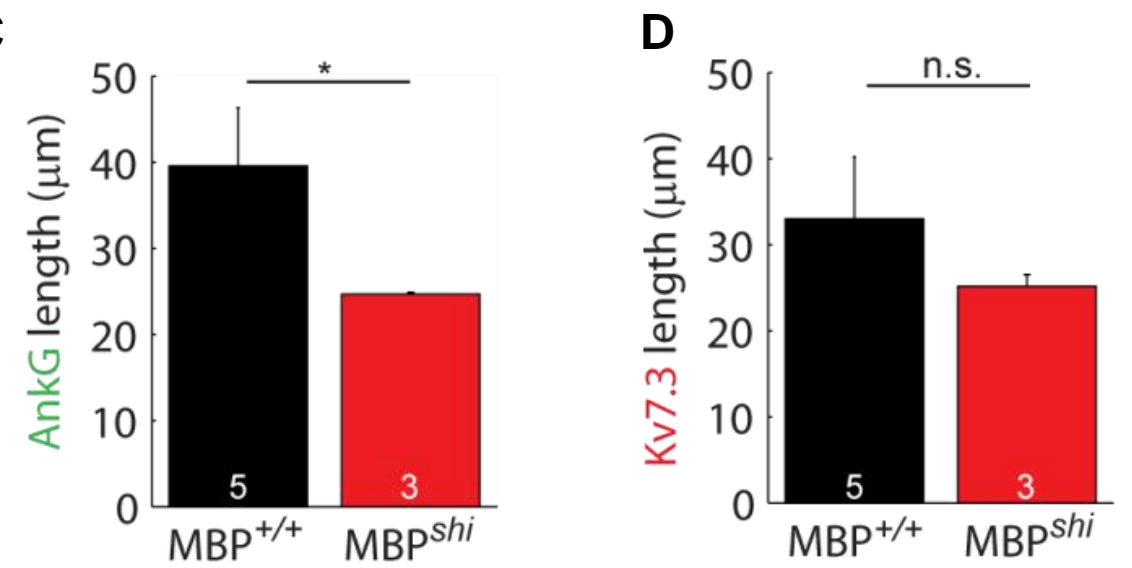

Figure 20. Dysmyelination generates a reduction in the length of the axon initial segment. Stainings of axon-initial segment proteins were done in mouse auditory cortex (layer II/III). Both AnkyrinG and $K_{v} 7.3$ channels were stained as a measure of the axon initial segment length for controls $(A)$ and $\mathrm{MBP}^{\text {shi }}$ mice (B). Below the images, blowups of AIS examples are seen for both AnkG (green), Kv 7.3 (red) and the merge. C) Quantification of AnkG showed a significant difference between groups $(p=0.035)$ while $D)$ quantification of $K_{v} 7.3$ did not $(p=0.57)$. For display purposes the background fluorescence was subtracted from the whole image. A ROI without an AIS was selected, the value measured and subsequently subtracted. $n=5 \mathrm{MBP}^{+/+}$, 20-55 AIS per mouse. $\mathrm{n}=3 \mathrm{MBP}^{\text {shi }}, 20-30$ AIS per mouse. 


\section{A comparison with white matter tracts}

Since the basic assessment of gray matter abnormalities in $\mathrm{MBP}^{\text {shi }}$ mice was done in the auditory system, I was curious whether these abnormalities would be replicable in white matter areas, and also, my interest was to obtain a real value of conduction velocity reduction in the mutants tested. For that reason, to assess the activity of optic nerve (ON) axons I recorded the compound action potential (CAP) of ON from control and MBP ${ }^{\text {shi }}$ mice (see Materials and Methods, section II.VII). These recordings were done in collaboration with Andrea Trevisiol. In Figure $21 \mathrm{~A}$, we can observe the typical triphasic CAP shape in which each peak corresponds, from left to right, to the activity of large, medium and small caliber axons, respectively.

As seen in the trace from the $\mathrm{MBP}^{\text {shi }}$ mice (Figure 21-A), there is an evident reduction in amplitude and a strong increase in latency. Also, the triphasic shape of the CAP is lost, becoming biphasic. Since it has been reported that $\mathrm{MBP}^{\text {shi }}$ mice have a higher number of small caliber axons compared to control animals (Kirkpatrick et al., 2001), the lack of wave I in the CAP of the optic nerve might have to do with a reduction in the number of middle or large caliber axons in the $\mathrm{MBP}^{\text {shi }}$ mice.

In addition, there is an evident hyperpolarizing phase that is non-existent in control animals. To quantify the effects seen here, I started by assessing the extent of conduction velocity deficit that results from the absence of MBP. 
A

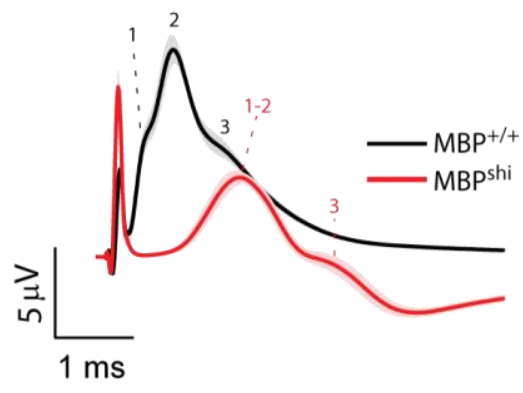

D

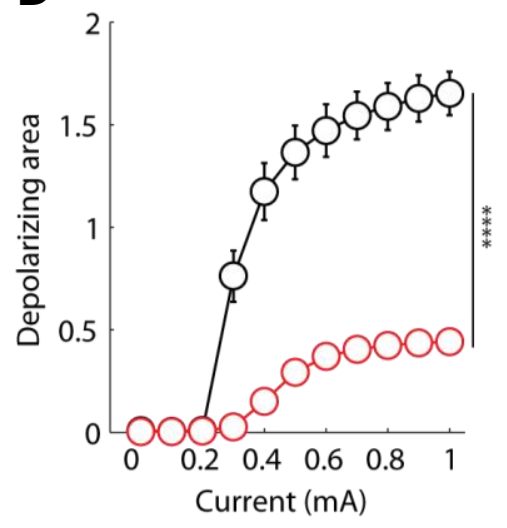

B

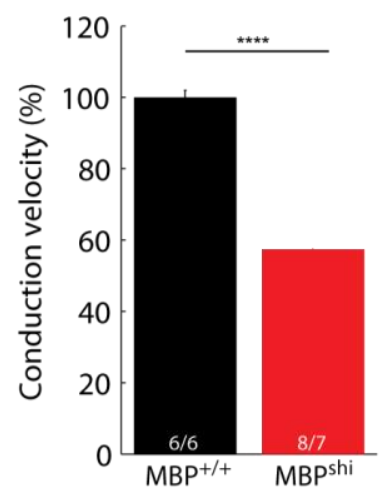

$\mathbf{E}$

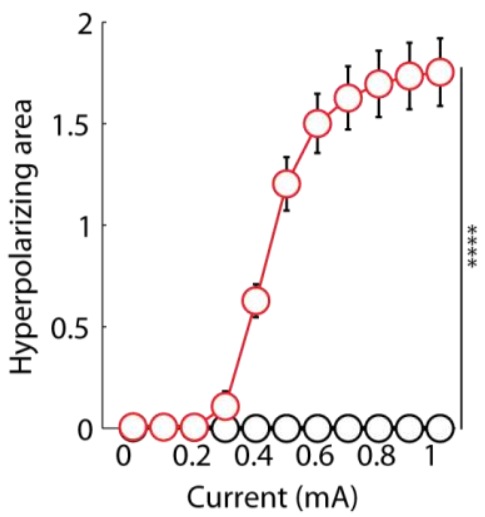

Figure 21. White matter abnormalities arise from the complete loss of MBP.

A) Compound action potentials from the optic nerve of $\mathrm{MBP}^{\text {shi }}$ mice (red) and control (black) at a stimulation intensity of $1 \mathrm{~mA}$. Traces show the mean of all optic nerves pulled together; shadow around the mean depicts the S.E.M. The typical triphasic CAP seen in control animals becomes biphasic in mutants, and there is a strong reduction of the response amplitude, accompanied by a strong hyperpolarizing phase. B) Conduction velocity was measured at an intensity of $0.7 \mathrm{~mA}$ stimulation MBP shi mice show a strong reduction in conduction velocity $(p<0.0001)$. C) Excitability of $\mathrm{MBP}^{s h i}$ is suboptimal, showing a significant difference in the amount of current is needed for the activity of MBPshi axons $(p<0.0001)$. D) Significant decrease in the depolarizing area $(p<0.0001)$ and $F)$ significant increase in the hyperpolarizing phase $(p<0.0001)$ of MBP ${ }^{\text {shi }}$. $n=8$ nerves from 7 control animals and $n=6$ nerves from $6 \mathrm{MBP}^{\text {shi }}$ animals. All measures show the mean and error bars the S.E.M. 
I measured the percentage of conduction velocity change with respect to the control animals and, as expected, the CAP is delayed about $0.9 \mathrm{~ms}$ from the control peak. I observed a significant reduction in conduction velocity $(\mathrm{CV})$ in $\mathrm{MBP}^{\text {shi }}$ mice $(\mathrm{p}<0.0001)$ (Figure 21-B), of approximately $42 \%$. To quantify nerve excitability, I measured the voltage output to increasing current steps. In Figure 21-C, we observe that $\mathrm{MBP}^{\text {shi }}$ animals require significantly higher currents to efficiently elicit a CAP (threshold is around $0.3 \mathrm{~mA}$ for control animals, while it is $0.4 \mathrm{~mA}$ for $\mathrm{MBP}^{s h}$ ). It has been reported that axons with different diameter have different excitability thresholds and that axons from $\mathrm{MBP}^{\text {shi }}$ spinal cord are less excitable than controls (Sinha et al., 2006), which suggests that the changes in excitability seen in the MBP ${ }^{\text {shi }}$ might have to do with changes in the distribution of axonal calibers. The statistical analysis confirmed a significant effect of stimulation frequency (ANOVA, $F(9,150)=639.86, p<0.0001$ ), and a strong effect of group (ANOVA, $F(1,150)=68.01, p<0.0001$ ) with an interaction (ANOVA, $F(9,150)=24.52, p<0.0001) . M B P^{s h i}$ CAPs showed significantly smaller amplitudes and area of the depolarizing peak (Figure 21-A and D), which means lower amount of axons are activated by the stimulation or there are conduction blocks. The statistical analysis confirmed a significant effect of stimulation frequency on the depolarizing are (ANOVA, $F(9,150)=99.51, p<0.0001$ ), and a strong effect of group (ANOVA, $F(1,150)=707.28$, $p<0.0001$ ) with an interaction (ANOVA, $F(9,150)=35.43, p<0.0001$ ).

In addition, $\mathrm{MBP}^{\text {shi }} \mathrm{CAPs}$ show a very strong hyperpolarization phase that is almost as large (in amplitude) as the depolarization phase (Figure 21-A and E). A significant effect of stimulation frequency in the area of hyperpolarization was seen (ANOVA, $F(9,150)=32.82$, $p<0.0001$ ), and a strong effect of group (ANOVA, $F(1,150)=397.63, p<0.0001$ ) with an interaction $(A N V O A, F(9,150)=33.07, p<0.0001)$.

Once it was clear that $\mathrm{MBP}^{\text {shi }}$ mice indeed showed less myelin in auditory areas, that gray matter areas had increased response latencies without impaired peripheral processing or changes in frequency tuning and that these effects paralleled the level of white matter conduction velocity decrease, I then tested more complex auditory processing, specially related to features needed for high-order cortical processing. 


\subsubsection{Lack of myelin impairs temporal processing in the auditory cortex}

The arrival time of different signals is an essential factor for the accurate and efficient information processing in the brain and timing is essential when it comes to the processing and perception of sound. The auditory system is the sensory system that requires the highest temporal precision, with processes happening even at the sub-millisecond range (Carr et al., 2001). Differential myelination is responsible for the reduction of variability of spike arrival times in the auditory system (Salami et al., 2003). It seems that myelin plays an important role in this sensory system, both to maintain proper conduction velocity along the stations, and to modulate temporal processes. Because of this, I was interested in testing how temporal aspects of hearing could be affected by dysmyelination.

\section{Is temporal acuity affected with dysmyelination?}

The first temporal aspect I addressed was temporal acuity by using a gap-detection paradigm. Gap-detection refers to the ability of detecting silent gaps in an otherwise continuous sound (Eggermont and Wang, 2011) and it's a commonly used measure to test temporal acuity in humans and animal models (Anderson and Linden, 2016; Irwin et al., 1981; Shailer and Moore, 1983). This property plays an essential role in speech discrimination (see section 1.1.2.2). I used a paradigm of gap-detection (see Materials and Methods, section II.V.V.III) to test temporal acuity in $\mathrm{MBP}^{\text {shi }}$ mice. An initial $200 \mathrm{~ms}$ BBN was followed by certain gap length, and then a second BBN of 50 ms. Responses to the second BBN (post-gap sound) were then compared to the baseline activity of that specific recording, taking a window of $50 \mathrm{~ms}$ before the initial BBN (pre-gap sound). The comparison yielded a series of $p$-values per recording for the different gaps.

We can observe in Figure 22 that $\mathrm{MBP}^{\text {shi }}$ mice presented a strong impairment of temporal acuity in the ACx. An individual example of a control and a MBP ${ }^{\text {shi }}$ recording exemplifies that $\mathrm{MBP}^{\text {shi }}$ mice have less spiking activity to the post-gap sound when the gap is small (Figure 22-A). In Figure 22-B, we can observe the mean responses in the form of PSTH for all the animals recorded in a window representing the pre-gap sound and the post-gap sounds for gaps below $5 \mathrm{~ms}$. 
A
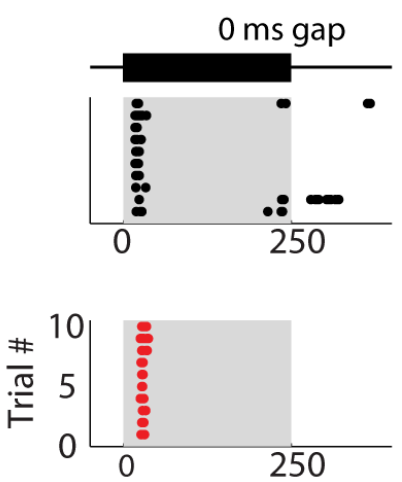
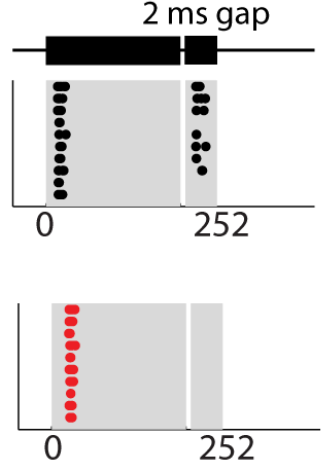

C

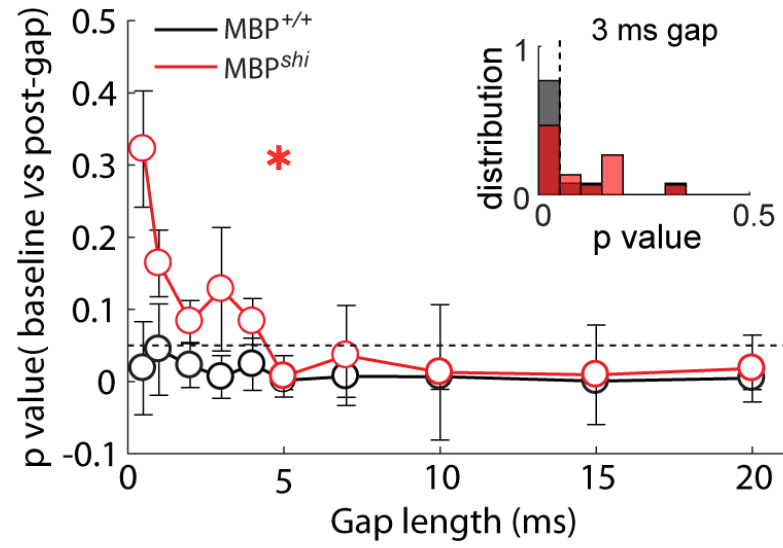

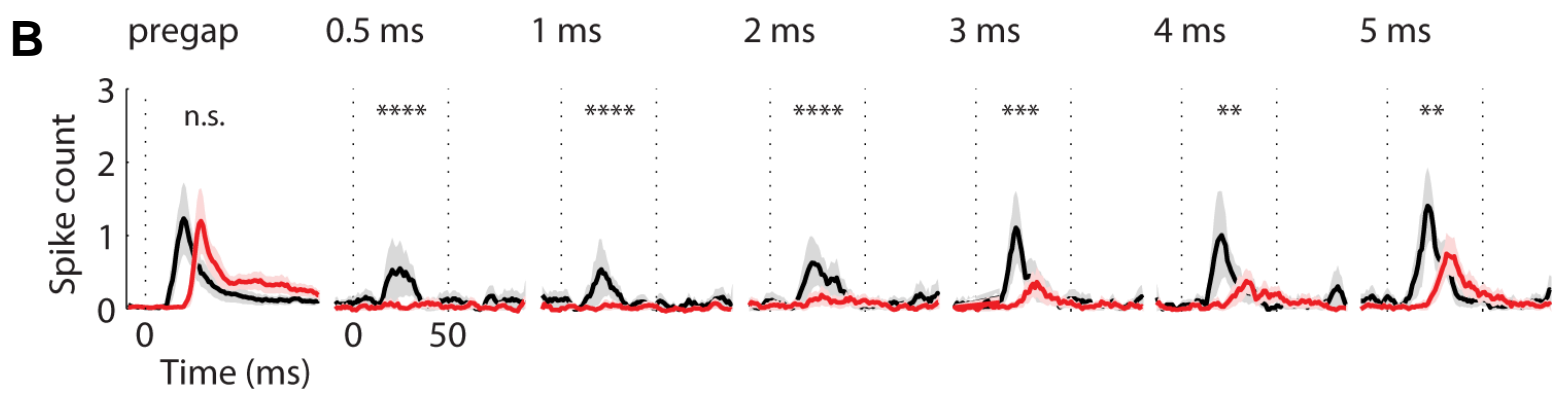

Figure 22. $\mathrm{MBP}^{\text {shl }}$ mice have impaired temporal acuity in the auditory cortex.

A) An individual example of the gap-detection recordings of a control (upper, black) and mutant (lower, red) mouse. These raster plots represent the spiking activity (individual dots) that occurs during sound presentation (gray patches) to the 10 stimulus repetitions. Above the raster plots, a representation of the sounds played is done in black bars, for a sound without a gap (0 ms gap, left side) and a sound with a $2 \mathrm{~ms}$ gap (right side). B) The average PSTH of all animals is depicted for control (balck) and mutants (red) with the S.E.M. represented as a shaded area following the same color code. Pre-gap and post-gap responses to the presentation of 0.5 to $5 \mathrm{~ms}$ gaps are represented. Significant differences were seen between groups for the PSTH comparison throughout the gaps (ANOVA, $p<0.0001$ ). $n=12$ control and $n=14$ mutant mice. C) Quantification of the differences between baseline and post-gap activity ( $50 \mathrm{~ms}$ window). A dotted black line depicts the threshold of $p=0.05$ for significance. The longer the gap length, the higher the significance value. There is a significant difference for gaps (KruskalWallis test, $p<0.0001$ ) and between groups (Kruskal-Wallis test, $p<0.0001$ ). The plot shows the median values for both groups, error bars show the standard error of the median. The internal distribution plot in B) shows all the $p$ value distributions for the $3 \mathrm{~ms}$ gap condition in control (gray) and mutant (red) mice. A vertical black dotted line depicts the $p$ value corresponding to 0.05 significance. It is possible to see that most of the control responses to the 3 ms gap are significantly different than baseline activity (left side of the vertical line), while responses from mutant mice tend to have a wider distribution. $n=12-15$ recording sites of 12 control animals and $n=14-20$ recordings of $14 \mathrm{MBP}^{\text {shi }}$ mice. 
We observe that pre-gap responses are not significantly different than control animals, amplitude wise (ANOVA, $F(1,462)=0.53, \mathrm{p}=0.46$ ) (Figure 22-C). This allowed the comparison between groups. It can be seen clearly, that $\mathrm{MBP}^{\text {shi }}$ mice do not respond to gaps below $3 \mathrm{~ms}$, and after, the responses are significantly smaller compared to control animals. Overall, I saw a significant effect of gap (ANOVA, $F(5,2872)=21.35, p<0.0001$ ), and group (ANOVA, $F(1,2872)=101.87, p<0.0001$ ), but no interaction (ANOVA, $F(5,2872)=0.15, p=0.98$ ) (Figure 22-C). Individual comparisons for each gap showed that the significant difference between groups was decreasing with the increase in gap (ANOVA, $p<0.0001, p<0.0001, p<0.0001$, $p=0.0002, p=0.0014, p=0.006$ for gaps from $0.5 \mathrm{~ms}$ to $5 \mathrm{~ms}$ respectively), indicating that, when presented with longer gaps, $\mathrm{MBP}^{\text {shi }}$ mice started to show more spiking activity to the post-gap sound, similar to control animals, but nevertheless with a lower amplitude at gaps below 5 ms.

When performing an analysis that instead of comparing between groups, the comparison was internal, between the baseline activity of each recording and the post-gap window (with a paired t-test), I observed very similar effects. In this case, it is expected that the longer the gap, the stronger the significance value is, meaning that longer gaps, elicited stronger post-gap responses, which was reflected in a lower p-value. In Figure 22-B we can clearly see this trend for both control and mutant animals. Also, I observed a significant effect of gap overall (Kruskal-Wallis test, $\left.x^{2}(9, n=316)=41.44, p=4.1 e^{-6}\right)$, a significant effect of group (Kruskal-Wallis test, $\left.x^{2}(1, n=316)=23.55, p=1.2 e^{-6}\right)$, and no differences in the amount of baseline activity $(p=0.18)$ between groups. These results confirm that, first of all, the spiking probability to the second sound increases with the length of the gap presented, and second, the $\mathrm{MBP}^{\text {shi }}$ mice tend to have reduced spiking probability after the gap, when the gap was lower than $\sim 4 \mathrm{~ms}$, showing similar-to-baseline activity.

\section{Is rate coding affected with dysmyelination?}

Another aspect of the assessment of auditory temporal processing is the effect that continuous stimulus repetition can have in the reliable firing of neurons. As noted in the introduction of this chapter (section 1.1.2.1), rate coding varies along the auditory pathway, being the ACx the station with the lowest coding upper limit, of around $15 \mathrm{~Hz}$ (Creutzfeldt et al., 1980; Wehr and Metherate, 2011). It has been reported that in the Long Evans shaker rat, a known CNS myelin mutant model that lacks MBP (Kwiecien et al., 1998), the calyx of Held synapse (an unusually large axo-somatic synapse located in the auditory brainstem) shows a 
reduction of firing reliability to high frequency stimulation (>300 Hz) (Kim et al., 2013a). To test if a possible reduction in reliability, due to the loss of myelin, might impact cortical processing, I recorded the neuronal response to click trains at different rates to assess rate coding in the auditory cortex.

A
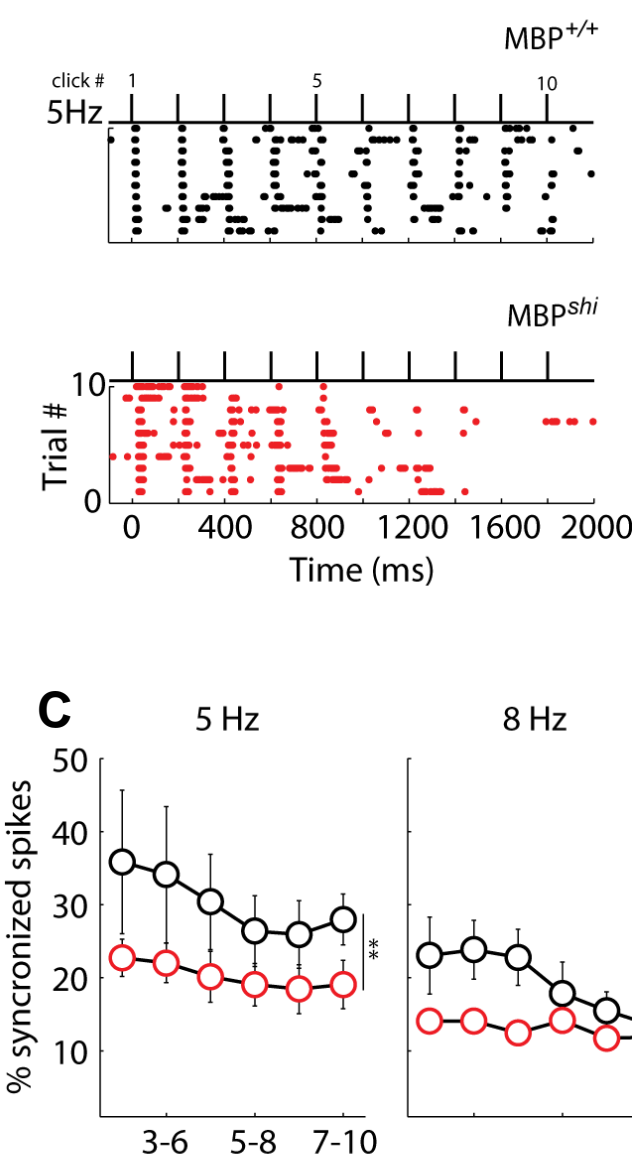

$8 \mathrm{~Hz}$

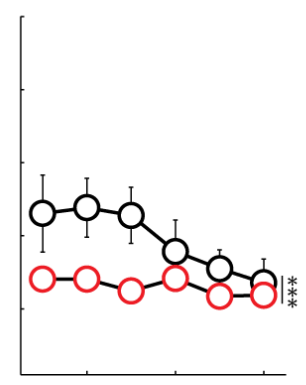

B

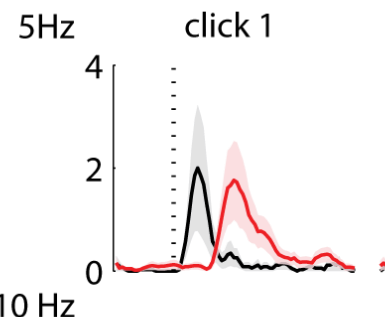

click 5

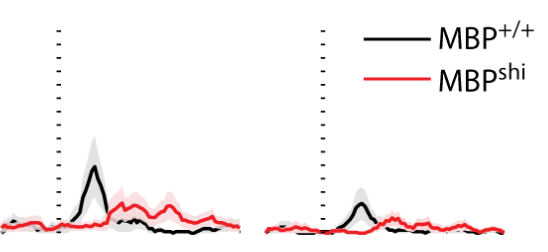

$10 \mathrm{~Hz}$

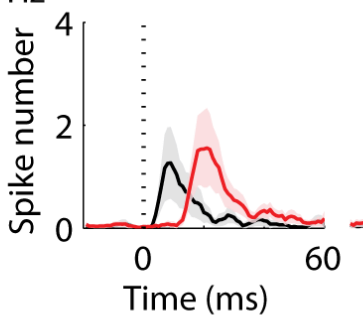

$10 \mathrm{~Hz}$

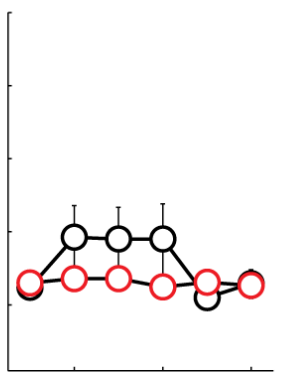

$14 \mathrm{~Hz}$

$20 \mathrm{~Hz}$

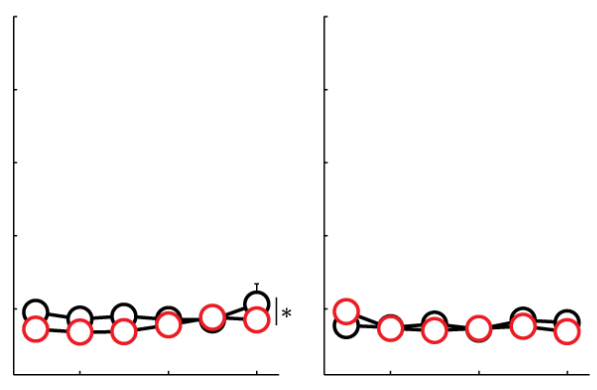

\section{Sliding window}

(click number)

Figure 23. Temporal reliability is affected in $\mathrm{MBP}^{s h t}$ mice.

A) Shows an example raster plot of a control animal (upper, black) and a MBP ${ }^{\text {shi }}$ mouse (lower, red) in response to a set of 10 clicks depicted by the vertical bars above the raster plots. These examples correspond to the condition of 5 $\mathrm{Hz}$, in which each stimulus was $200 \mathrm{~ms}$ apart. It is possible to observe that it is a common feature of auditory cortex neurons to present certain level of adaptation, showing less amount of spiking activity by click 10 compared to the initial clicks, nevertheless, in the mutant animals we see a steeper decay on spiking activity by click 6 . In B) we can see the mean PSTH of all animals recorded for control and mutant animals (conserving the same color-code). A blowup of click 1, 5 and 10 (as depicted in A) is shown. Thick lines show the mean of all animals recorded and shaded below each line is depicted the S.E.M. The upper panel shows the responses to $5 \mathrm{~Hz}$ stimulation rate while the lower to $10 \mathrm{~Hz}$. While responses in control and mutant animals are similar in amplitude to the first peak, a strong reduction of response amplitude is seen in $\mathrm{MBP}^{\text {shi }}$ mice with increasing clicks. $\mathrm{n}=10$ control and $\mathrm{n}=13$ mutant animals. C) Quantification of spike synchrony showed that there is a significant difference between control and mutant mice (ANOVA, $p=0.0001$ ) through the frequencies presented. $n=3-6$ control animals and $n=6-9$ mutant animals. 
In Figure 23-A, we can observe an example raster-plot of a $\mathrm{MBP}^{+/+}$mouse and a $\mathrm{MBP}^{\text {shi }}$ mouse to a set of 10 clicks at a rate of $5 \mathrm{~Hz}$. It is possible to see already in this individual animal example, that $\mathrm{MBP}^{\text {shi }}$ mice show a reduction in spiking activity after the $5^{\text {th }}$ click presentation within each trial. In Figure 23-B, one can see the mean PSTH for both groups evoked by the $1^{\text {st }}, 5^{\text {th }}$ and $10^{\text {th }}$ clicks at two different repetition rates $(5$ and $10 \mathrm{~Hz})$. It is possible to observe that the initial click response is not different in amplitude (similar to Figure 18-A where the response to the first click was used to measure latencies and amplitudes). Nevertheless, MBP $^{\text {shi }}$ responses decay faster than control ones (see response at click 5 and 10 for the condition of $5 \mathrm{~Hz}$ ). A common way of measuring the strength of spike synchrony is to use circular statistics (see Materials and Methods, section II.V.VI.VI). Briefly, all spikes happening in the 10 stimulus repetitions are ordered by their latency relative to the previous stimulus onset. I divided the responses to different sets of 4 clicks to understand the dependency of click number in the spike probability. The leftmost graph in Figure 23-C corresponds to the mean percentage of synchronized spikes when the neurons were stimulated with a train of 10 clicks at $5 \mathrm{~Hz}$. The $x$ axis represents the sliding window used every 4 clicks (namely, clicks 2-5, 3-6, 4-7, 5-8, 6-9, 7-10). This same procedure was repeated for all the rates presented.

First of all, we can observe a strong dependency of spike synchrony and click rate, eliciting the highest rates $(14$ and $20 \mathrm{~Hz}$ ) lower levels of synchrony. I observed a significant effect of rate (ANOVA, $F(4,274)=44.49, p<0.0001$ ), and group (ANOVA, $F(1,274)=16.34$, $p=0.0001$ ) together with a significant interaction (ANOVA, $F(4,274)=3.76, p<0.0054$ ), but no effect of click number (ANOVA, $F(5,274)=0.85, p<0.51$ ). Individual rate comparisons showed that the main effects were found at 5 and $8 \mathrm{~Hz}$ (ANOVA, $p=0.0021$ and $p=0.0002$ respectively). These results show that $\mathrm{MBP}^{\text {shi }}$ mice present reduced rate coding in the $\mathrm{ACx}$.

\section{The partial effects of rate coding deficits in high-order processing: the case of deviance detection}

The everyday detection of relevant stimulus that might be harmful for an organism and filtering out the ones that are not is an essential process for survival. This process is called stimulus-specific adaptation (SSA), by which neurons adapt their responses to frequently presented stimulus (standard tone), while responding stronger to unexpected ones (deviant tone) (Pérez-González and Malmierca, 2014). This way, the system is wired to detect unexpected events and respond accordingly (Ayala and Malmierca, 2012; Nelken, 2014; 
Taaseh et al., 2011). It has been proposed this represents a type of 'primitive intelligence' (Näätänen et al., 2001). The majority of primary cortical neurons have SSA (Natan et al., 2015) and this feature has been proposed as the mechanism for deviance detection along the auditory system (Ulanovsky et al., 2003) and since it requires the storage of short-term information, it is associated with a type of 'primitive intelligence' (Näätänen et al., 2001).
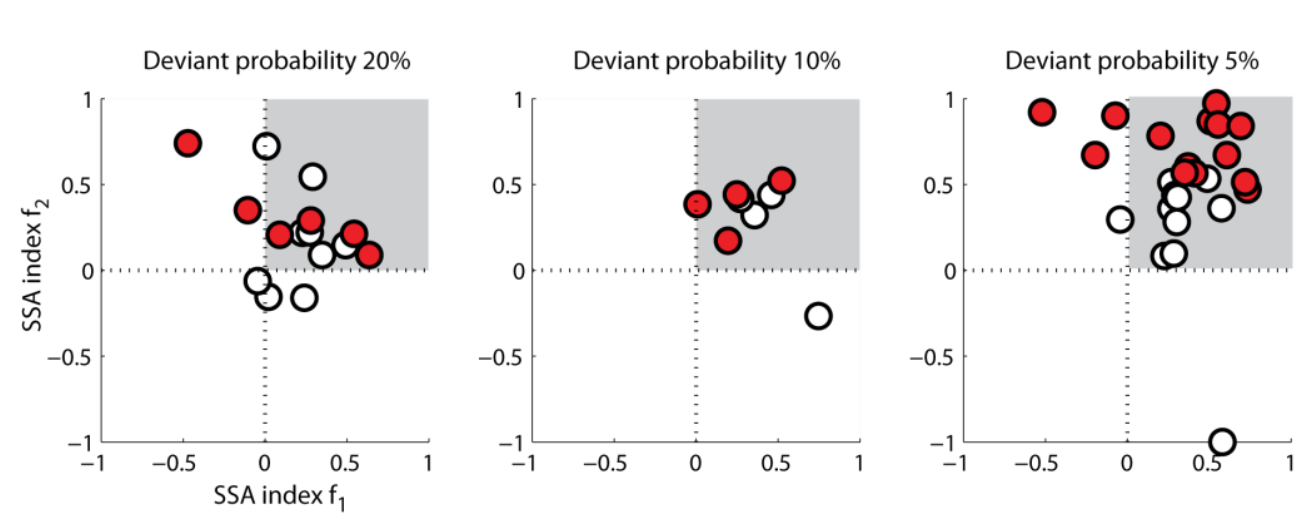

Figure 24. Deviance detection is potentiated in $\mathrm{MBP}^{\text {shi }}$ mice.

Stimulus specific adaptation was measured as a high-order processing task that requires the fast comparison of continuously presented stimuli. Using a presentation rate of $3 \mathrm{~Hz}$ and a $\Delta \mathrm{F}$ of $10 \%$, deviant probability was 20 (left), 10 (middle) and 5\%(right) for control (white circles) and MBP shi mice (red circles).Each circle represents one recording. As the deviant probability gets smaller the SSA indices grow. Strong SSA is seen in the upper-right corner (gray area). MBP ${ }^{\text {shi }}$ mice seem to have stronger SSA indices compared to control mice. $n=4-11$ recordings for control and $n=4-13$ recordings for mutant mice.

To find a process that requires high-order cortical processing and it is related to the capabilities of rate coding, I measured SSA in cortical neurons in the MBP ${ }^{\text {shi }}$ mice using an oddball paradigm. I presented the stimuli at $3 \mathrm{~Hz}$ and with a difference of $10 \%$ between frequencies. Deviant probability was 20, 10, or 5\% (Figure 24). Preliminary data suggest additionally to what was observed in frequency tuning, that $\mathrm{MBP}^{\text {shi }}$ do not have a problem detecting and differentiating two frequencies, since they did show SSA. In addition, these data suggest that $\mathrm{MBP}^{\text {shi }}$ mice have stronger SSA indices compared to control animals. One factor that could be contributing to this would be the strong adaptability seen when continuous stimulation is presented. This suggests that the rate coding abnormalities seen in MBP ${ }^{\text {shi }}$ mice might be affecting high-order cortical processing as seen by deviance detection deficits. 


\subsubsection{Impaired axonal firing at high stimulation rates in $\mathrm{MBP}^{\text {shi }}$ white matter}

Since the impairment in rate coding seen in the auditory cortex might have similar mechanisms to those of reduced firing with high rate simulation white matter tracts, I collaborated with Andrea Trevisiol, to do optic nerve recoridngs in the $\mathrm{MBP}^{\text {shi }}$ mice during high rate stimulation. In addition, this system is very appropiate to do pharmacology and understand how dysmyelination is affecting the CAP at different levels. As previoulsy described I recorded the CAP of optic nerves from $\mathrm{MBP}^{\text {shi }}$ mice. In this case, a ramp stimulation protocol in which stimulation rates were applied progresivelly from 1 to $100 \mathrm{~Hz}$ was used. All analysis were perfromed without taking into account the $1 \mathrm{~Hz}$ stimulation rate, since it did not show differences from baseline activity. It is known that high rate stimulation causes a reduction in the CAP area and amplitude (Brown et al., 2003; Saab et al., 2016), which was also observed here (ANOVA, $F(6,56)=21.46, p<0.0001$ ). In Figure 25 we can observe the normalized measure of amplitude decrease of the CAP, recorded from optic nerve of control and $\mathrm{MBP}^{\text {shi }}$ mice. The variability of $\mathrm{MBP}^{\text {shi }}$ mice was larger than the one of controls, probably due to the stability of dysmyelinated optic nerves within the recording setup.

In addition, $\mathrm{MBP}^{\text {shi }} \mathrm{ON}$ showed strong changes in the CAP shape with the increase in stimulation rate. First of all, $\mathrm{MBP}^{\text {shi }} \mathrm{CAPs}$ had a reduction of their hyperpolarization phase with stimulation. This effect can be seen in the CAP shape exemplification above the $15 \mathrm{~Hz}$ condition as compared to the $4 \mathrm{~Hz}$. A strong fall in $\mathrm{MBP}^{\text {shi }} \mathrm{CAP}$ amplitude was observed between $50 \mathrm{~Hz}$ and $75 \mathrm{~Hz}$. The quantification of the strongest decay in amplitude for both groups yielded a singificant effect at $75 \mathrm{~Hz}(p=0.0095)$. This means that at this stimulation rate, $\mathrm{MBP}^{\text {shi }}$ axons show stronger decay in amplitude compared to controls. Interestingly, at $100 \mathrm{~Hz}$, the CAP amplitude of MBP ${ }^{\text {shi }}$ ON increased, altough it was not significant compared to control animals $(p=0.91)$. This effect might have to do with the fact that at $100 \mathrm{~Hz}$ the $\mathrm{MBP}^{\text {shi }} \mathrm{CAP}$ showed a strong decrease in the hyperpolarizing phase, which in turn increased the whole area of the CAP, having an impact also in its amplitude. 
Once I observed that $\mathrm{MBP}^{\text {shi }}$ in white matter tracts show a similar phenotype than the one observed in cortical neurons, it was of relevance to elucidate the mechanisms that are regulating changes in excitability and fatigability in the $\mathrm{MBP}^{\text {shi }}$ model. For that reason, I used the same high-rate stimulation protocol in combination with 4-Aminopyridine (4-AP), a commontly used drug to treat Multiple Sclerosis. 4-AP is a selective and reversible (see Apendix Figure 50) blocker of the $\mathrm{K}_{\mathrm{v}} 1$ channel family. Since 4-AP restores the precition of spiking in a model of cerebellar ataxia, (Alviña and Khodakhah, 2010), I hypothesized that changes in excitability had to do with the activity of $K_{v} 1.1$ and $K_{v} 1.2$ channels being overexpressed in the optic nerves of $\mathrm{MBP}^{\text {shi }}$ mice. Because of that, I tested the effects of 4-AP

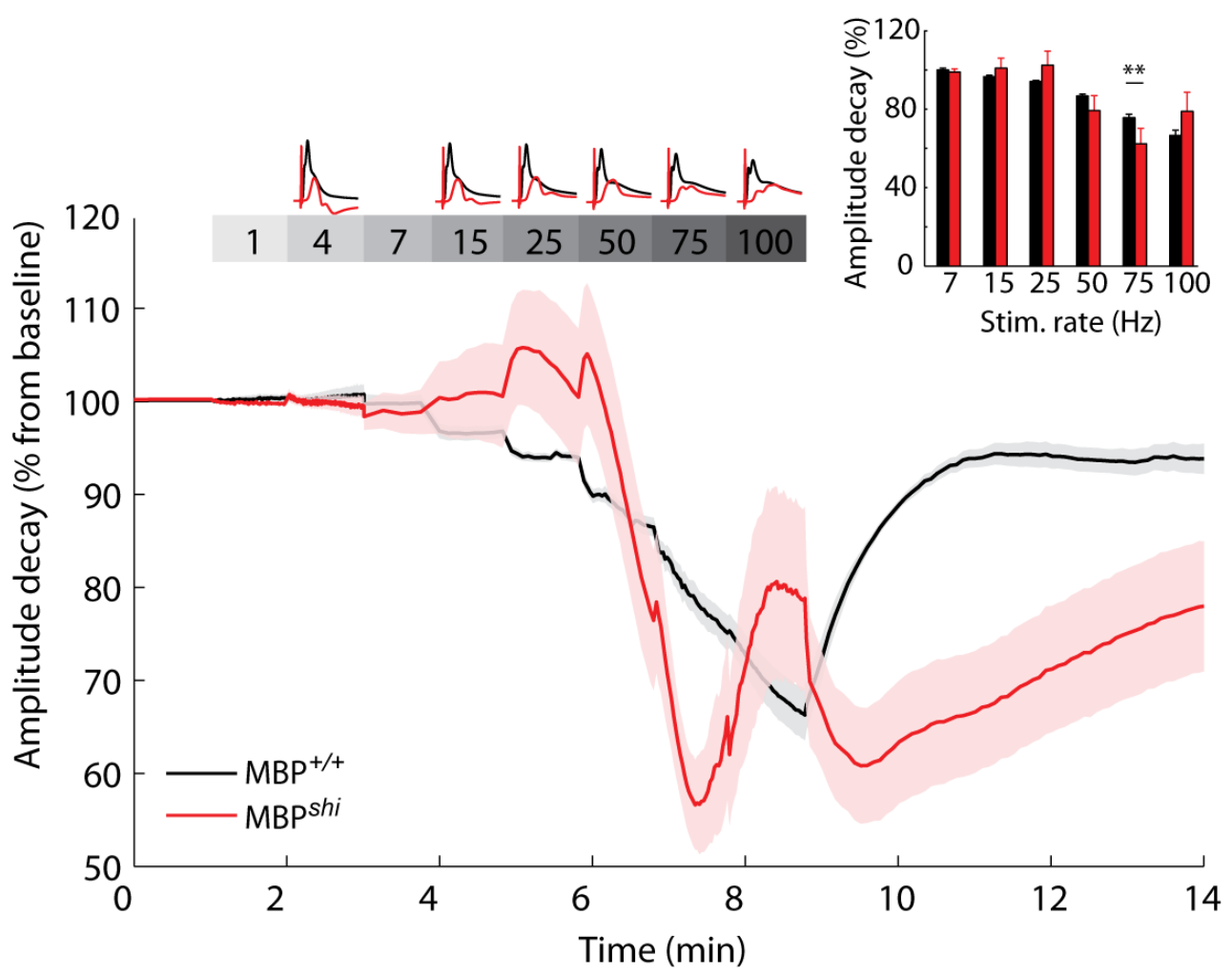

Figure 25. Axons from the white matter of the $\mathrm{MBP}^{s h l}$ mice show a strong decay when stimulated at high rates.

A ramped protocol of rate stimulation was applied to optic nerves of $\mathrm{MBP}^{\text {shi }}$ mice and control littermates. The maximum peak was obtained for each CAP at a specific time point as a measure of CAP amplitude. Responses were normalized to the maximum peak seen in CAPs during baseline activity. Control (black) and mutant (red) measurements were plotted for all the stimulation rates applied (gray squares representing rates). On top of the squares we can see examples of the shape of the CAP for each rate. In MBP ${ }^{\text {shi }}$ mice it was possible to see that at low stimulation rates, the CAP waveform does not change much, and in fact, the hyperpolarizing phase is reduced (see traces above the gray square representing $15 \mathrm{~Hz}$ ). We can clearly observe that there is a dependency of the CAP amplitude and the stimulation rate. Higher stimulation rates elicit stronger reductions in amplitude. When measuring the minimal response peak found for each stimulation rate, at $75 \mathrm{~Hz}$ I found a statistically significant decrease of the amplitude of MBP ${ }^{\text {shi }}$ CAPs compared with control animals $(p=0.0095) . n=4$ nerves of 4 control animals; $n=6$ nerves of 5 mutant animals. 
$(25 \mu \mathrm{M})$. A series of deses were tested in a pilot experiment (1, 10, 25, 50, 75 and $100 \mu \mathrm{M})$. No effects were seen with the $1 \mu \mathrm{M}$, and minimal effects were seen with $10 \mu \mathrm{M} 4$-AP. Doses of 75 and $100 \mu \mathrm{M}$ generated an unusall shift of the hyperpolarizing phase, wich became strongly depolarizing, an effect that was not seen in control animals. In addition, dosages of $50 \mu \mathrm{M}$ have been reported to be epileptogenic in slices (Chesnut and Swann, 1990). For that reason, I decided to use $25 \mu \mathrm{M}$, where a strong change to the CAP was induced, but it did not alter the typicall CAP waveform seen in controls. Very preliminary experiments $(n=2$, no statistical anaysis performed) show that 4-AP effectively restores the hyperpolarizing phase amplitude and depolarizing area of the $\mathrm{MBP}^{\text {shi }}$ CAP (Figure 26-A, C, D). Nevertheless, the reduced excitability of $\mathrm{MBP}^{\text {shi }}$ nerves is not rescued (Figure 26-B). In addition, when applying 4-AP before the high-rate stimulation, the decay phenotype is not reestablished, and in fact, it seems as if 4-AP was potentiating the amplitude decay in $\mathrm{MBP}^{\text {shi }} \mathrm{ON}$ (Figure 26-E). The only effects that 4-AP was able to rescue were related to the strong hyperpolarization phase and reduced amplitude seen in $\mathrm{MBP}^{\text {shi }}$ nerves. These very preliminary results show that some of the changes seen in $\mathrm{MBP}^{\text {shi }}$ white matter might have to do with the overexpression or increased availability of $\mathrm{K}_{\mathrm{v}} 1$ channels. Nevertheless, the extrapolation of these effects to what was observed in gray matter must be taken carefully. 
A

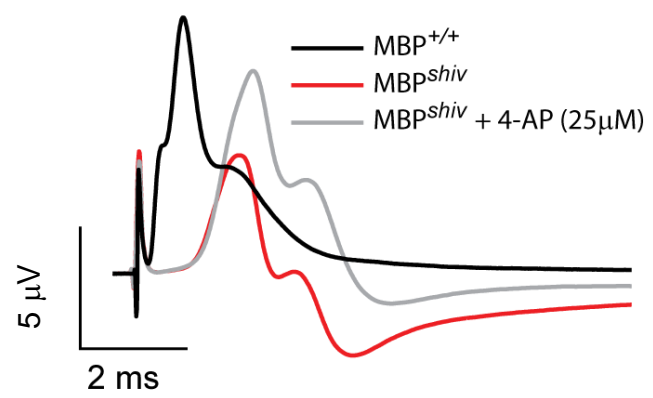

C

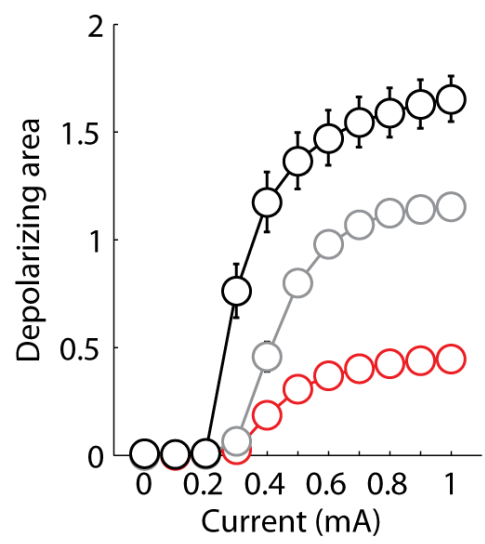

B
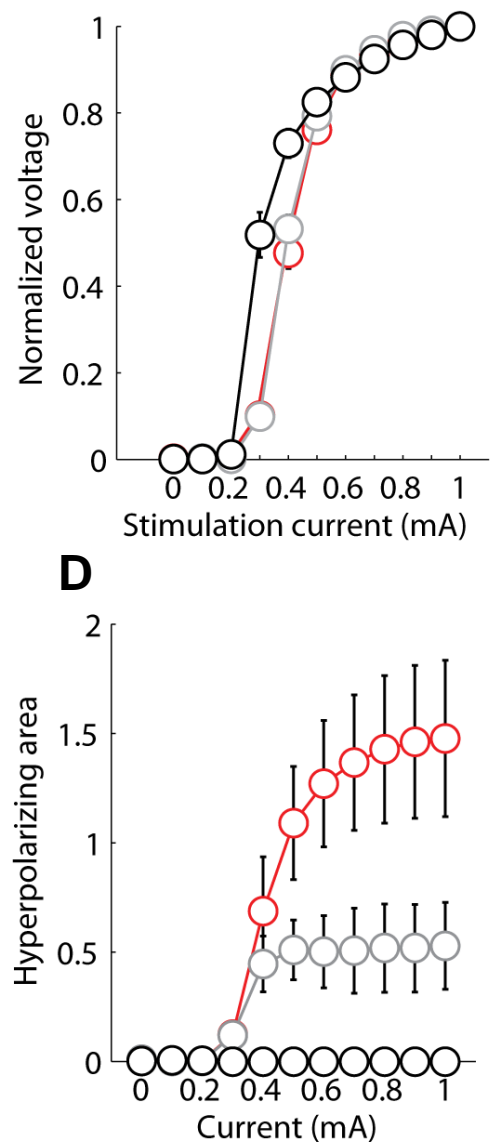

E

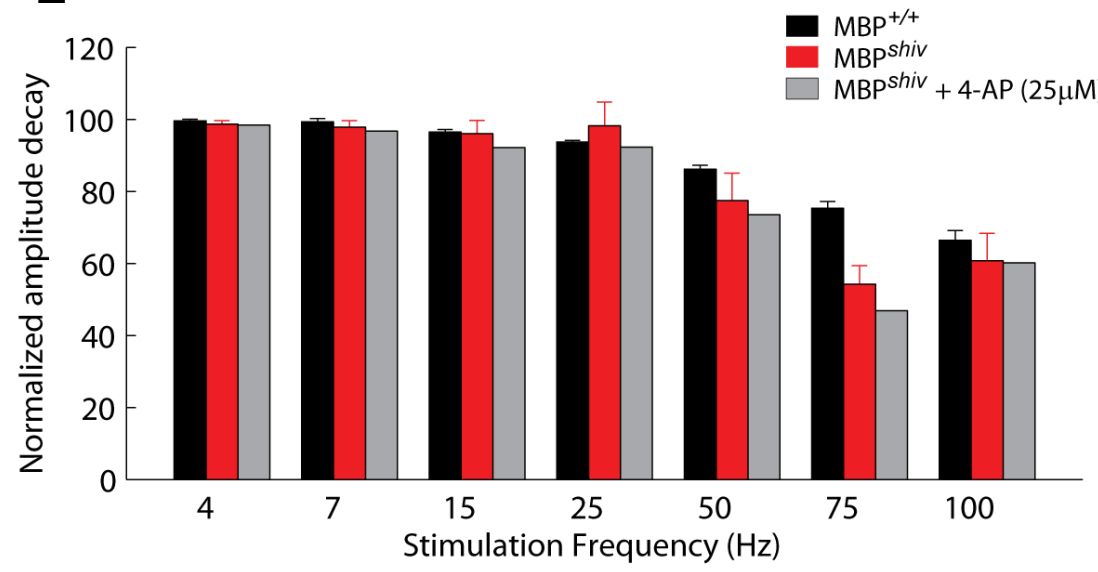

Figure 26. 4-AP applied to $\mathrm{MBP}^{s h t}$ optic nerve, rescues amplitude but not latency changes or fatigability.

A)Shows the CAP waveform of control (black), MBP ${ }^{\text {shi }}$ (red) and MBP shi $+4-A P(25 \mu M)$. B) The IV curve of the previous three conditions is shown. 4-AP does not change the reduced excitability of MBP ${ }^{\text {shi }}$ nerves. Both the depolarization area C) and the hyperpolarization area reduction seen in the MBP ${ }^{\text {shi }}$ nerves (red) D) are rescued with a strong trend towards the control condition with (black) 4-AP (gray). E) 4-AP does not change the fatigue phenotype seen in $\mathrm{MBP}^{\text {shi }}$ at $75 \mathrm{~Hz}$ stimulation rate. 


\subsubsection{Cortical-specific myelin loss impairs response amplitudes}

The auditory cortex is a complex auditory station where several essential processes take place for sound perception (Talwar et al., 2001). The ACx can send signals to other cortical areas and also projects to subcortical stations, by which it can modulate sound processing (Mellott et al., 2014). The ACx possess a heterogeneous cytoarchitecture, connectivity and spiking activity, and it keeps the classical cortical layered architecture. Cortical neurons from the layers I recorded ( II/III to IV) receive thalamic inputs and inputs from other cortical cells (Imaizumi and Lee, 2015). The importance of cortical demyelination has been highlighted in MS patients, as an added culprit of the neurological manifestations of this disease (Kutzelnigg et al., 2005). I was interested on assessing how an individual defect of dysmyelination in a specific circuit could impact auditory processing.

I used a novel mouse model, the MBP ${ }^{\mathrm{fl} / \mathrm{fl}} ; \mathrm{EMX1-cre}$ mice, that was generated by Wiebke Möbius from the Max Planck Institute of Experimental Medicine. These mice have a forebrain-restricted dysmyelination due to the EMX1 dependent cre expression. EMX1 is a transcription factor only expressed in cells confined to the forebrain (Gorski et al., 2002; Gulisano et al., 1996; Stocker and O'Leary, 2016). As a result, the expression of MBP would be knocked-out only in the forebrain, providing a perfect model to study the effects of circuitspecific dysmyelination. For simplicity reasons, these mutant animals will be called from now on $\mathrm{MBP}^{\mathrm{em} x}$.

\section{Basic hearing properties}

I started by measuring the subcortical responses in these mice, as a control to confirm the mutation was not affecting other brain areas besides the forebrain. The ABR shape of control and $\mathrm{MBP}^{\mathrm{em} x}$ animals overlap fully (Figure 27-A). No differences were seen in hearing thresholds $(p=0.36$ ), latencies (ANOVA, $p=0.057, p=0.12, p=0.33, p=0.44, p=0.1$ for waves I to $V$ respectively), or amplitudes (ANOVA, $p=0.96, p=0.4, p=0.99, p=0.63, p=0.08$ for waves I to $V$ respectively). Then I did extracellular MUA recordings from the auditory cortex, and observed no differences in the latency of response $(p=0.17)$ (Figure 27-E, F) but a strong decrease in amplitude $(p=0.0086)$ (Figure 27-E, $G$ ), suggesting the presence of conduction blocks due to dysmyelination of the latter thalamo-cortical projections. 
A

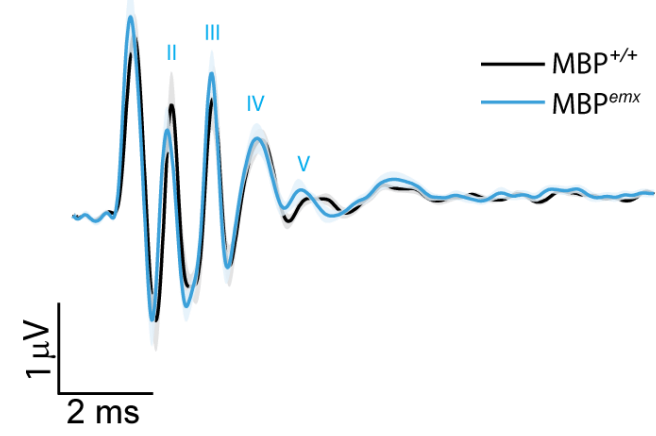

B

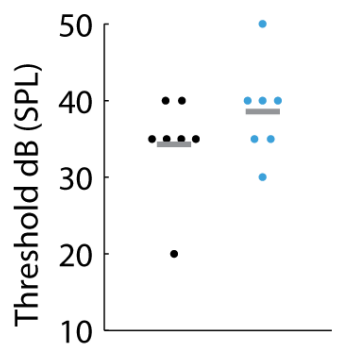

C

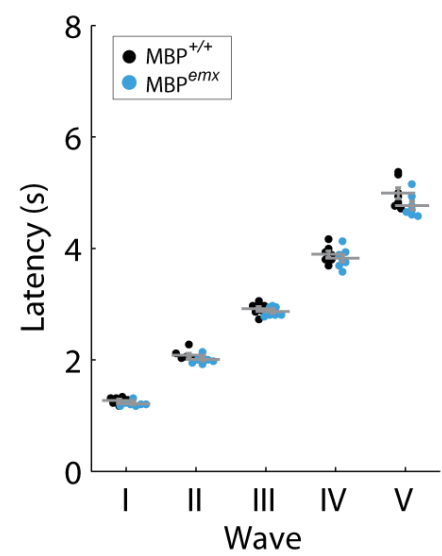

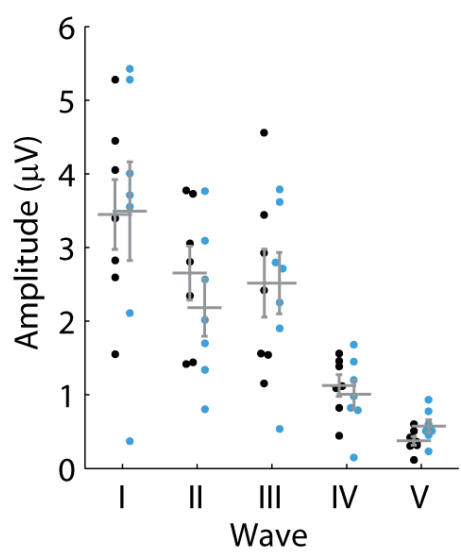

E

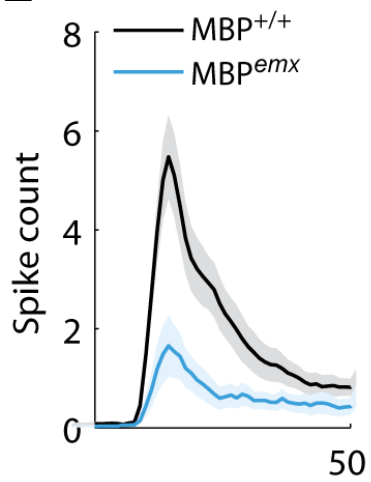

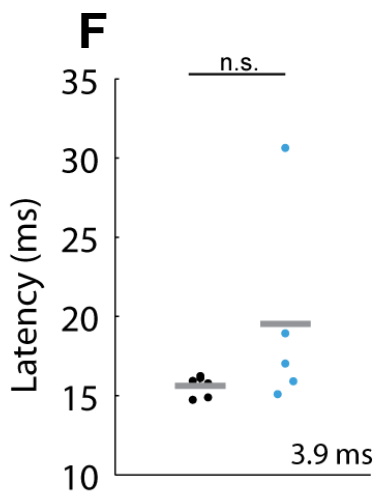

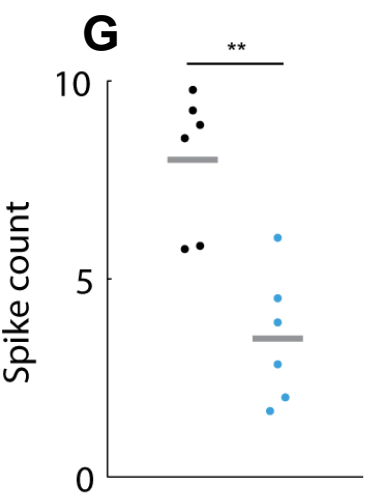

Time (ms)

Figure 27. Subcortical auditory processing is not impaired MBP $^{e m x}$ mice but a strong reduction in response amplitudes is seen in the cortex.

A) The 5 characteristic waves of ABRs, belonging to 5 different auditory stations are depicted, from the auditory nerve (I) until the inferior colliculus (V) for control (black) and mutant (blue) animals. $\mathrm{MBP}^{e m x}$ mice (blue) show no differences in auditory brainstem potentials elicited by clicks at $80 \mathrm{~dB}$. $B)$ No differences are seen at the threshold level $(p=0.36)$, and indirect measure of outer hair cell function. C) No differences in latencies or amplitudes D) were observed in $\mathrm{MBP}^{e m x}$ animals, compared to control. E) PSTH of the auditory cortex response to a click stimulus for the average of control (black) and MBP ${ }^{\text {emx }}$ (blue) mice. A strong decrease in amplitude is seen at this level. Upon quantification, in F) it is possible to see no differences in response latencies between groups $(p=0.17)$; the number in the right lower corner depicts the difference between control and mutant means. Grey bars depict the mean per group. G) A significant decrease in response amplitude is seen for $M^{e m x}$ mice in the $A C x(p=0.0086) . n=6$ controls and $n=6$ mutant mice. 


\section{Is cortex-specific dysmyelination affecting temporal acuity?}

To further characterize auditory abnormalities that these cortical-specific dysmyelination mice might have, I used the gap-detection paradigm to assess temporal acuity. We can observe in Figure 28 that individual recording examples of $\mathrm{MBP}^{\mathrm{emx}}$ mice show normal gap-detection. When observing the pre-gap and post-gap PSTH, the MBP ${ }^{e m x}$ mice show strongly dampened responses already at the pre-gap sound (Figure 28-B). This strong effect did not allow the comparison of control and mutant responses, since the amplitude effect could not be dissociated. For that reason, I analyzed the detection of gaps doing an internal assessment per recording file by comparing the baseline activity with the post-gap activity. A set of $p$ values were obtained per file for all the gaps. The longer the gap, the smaller the $p$-value, which meant that the responses were stronger. As expected, I saw a strong correlation between the gap length and a reduction of the $p$-value, meaning that longer gaps, elicited stronger post-gap responses (in Figure 28-C) (Kruskal-Wallis test, $x^{2}(9, n=268)=32.29$, $p=0.0002)$. A significant effect of group was also observed (Kruskal-Wallis test, $x^{2}(1$, $n=268)=29.03, p=7.1 e^{-8}$ ). The statistical comparison at each gap length confirmed that the main effects are seen at gaps of 2, $4 \mathrm{~ms}$, also with lower recognition of $10 \mathrm{~ms}$ and $15 \mathrm{~ms}$ gap (Kruskal-Wallis test, $p=0.35, p=0.48, p=0.003, p=0.47, p=0.035, p=0.16, p=0.068, p=0.034$, $p=0.01, p=0.1$ for gaps from 0.5 to $20 \mathrm{~ms}$ respectively). In addition, no differences were seen between groups in the amount of baseline activity $(p=0.92)$. These results show that $\mathrm{MBP}^{\mathrm{em} x}$ mice tend to have reduced spiking probability after the gap, especially at gaps between 1 and $4 \mathrm{~ms}$ and thus had a subtle impairment of temporal acuity in the ACx. Overall, it seems that a cortico-restricted myelin deficiency impacts in a subtle manner temporal acuity; a very interesting observation since this means partially the defects that I observed in the MBP ${ }^{\text {shi }}$ mice at the cortical level are due to a cortical loos of temporal reliability due to dysmyelination. 
A
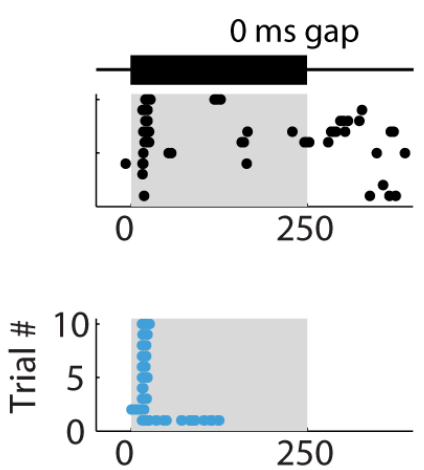

Time (ms)

\section{C}

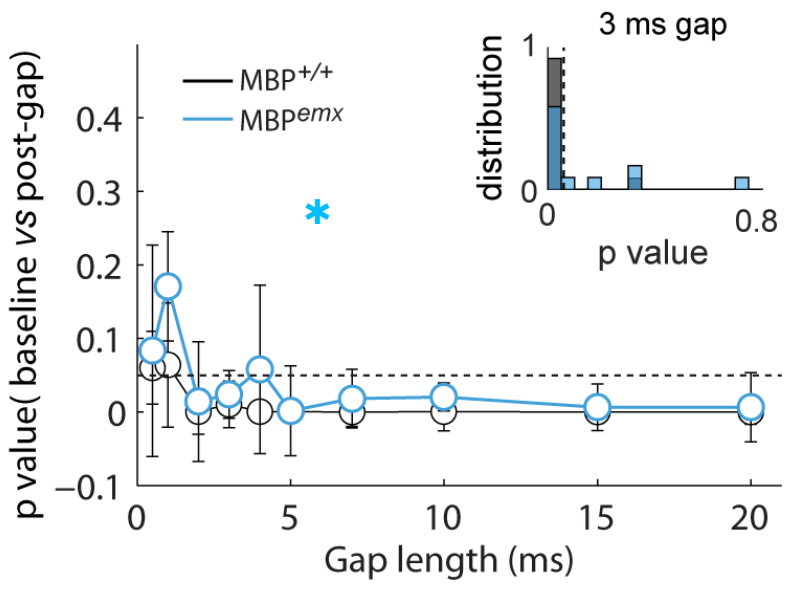

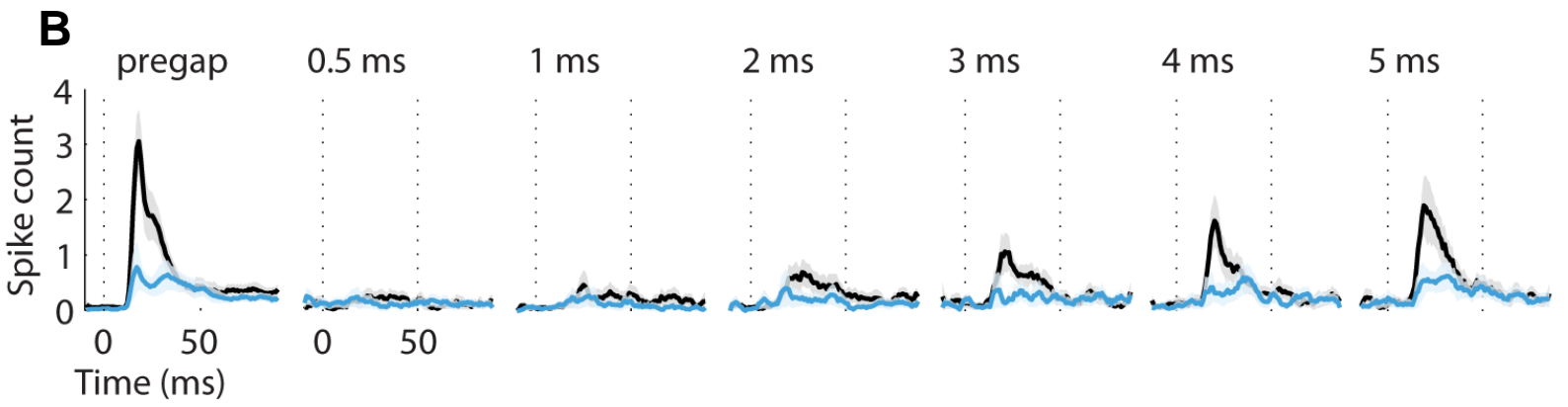

Figure 28. Impaired temporal acuity in $\mathrm{MBP}^{\mathrm{emx}}$ mice.

A) An individual example of the gap-detection recordings of a control (upper, black) and mutant (lower, blue) mouse. These raster plots represent the spiking activity (individual dots) that occurs during sound presentation (gray patches) to the 10 stimulus repetitions. Above the raster plots, a representation of the sounds played is presented in black bars, for a sound without a gap (0 ms gap, left side) and a sound with a $2 \mathrm{~ms}$ gap (right side). B) The average PSTH of all animals for control (black) and MBP ${ }^{\text {emx }}$ mice (blue) with the S.E.M. represented as a shaded area following the same color code. Pre-gap and post-gap responses to the presentation of 0.5 to $5 \mathrm{~ms}$ are shown. Significant differences were seen throughout the gaps (ANOVA, $p<0.0001$ ). Nevertheless, this cannot be entirely attributed to an actual loss of temporal acuity, but a generalized reduction in response amplitudes, as seen for the pre-gap. $n=6$ control and $n=6$ mutant mice. C) Quantification of the differences between baseline and post-gap activity (50 ms window). The longer the gap length, the higher the significance value. A dotted black line depicts the threshold of $p=0.05$ for significance. $A$ significant difference was seen for gaps (ANOVA, $p<0.0002$ ) and groups (ANOVA, $p=7.1 \mathrm{e}^{-8}$ ). The plot shows the median values for both groups, error bars show the standard error of the median. The internal distribution plot in $B$ ) shows all the p-value distributions for the $3 \mathrm{~ms}$ gap condition in control (gray) and mutant (green) mice. A vertical black dotted line depicts the $p$ value corresponding to 0.05 . It is possible to see that most of the responses of both the control and mutant to the $3 \mathrm{~ms}$ gap are significantly different than baseline activity (left side of the vertical line) and have similar distributions. $n=10-15$ recording sites of 6 control and $n=11-14$ recording sites of 6 mutant mice. 


\section{Is cortex-specific dysmyelination affecting rate coding?}

The other aspect of temporal processing that was tested, as seen before, was the ability of rate coding of cortical neurons. Rate coding was tested in the auditory cortex of $\mathrm{MBP}^{\mathrm{emx}}$ mice. In Figure 29-A, we can observe an example raster-plot of a $\mathrm{MBP}^{+/ t}$ and a $\mathrm{MBP}^{\mathrm{em} x}$ mouse where the reduced spiking synchrony is appreciated. Figure 29-B shows the mean of all recorded animals for the control group and the MBP ${ }^{e m x}$ mice for the $1^{\text {st }}, 5^{\text {th }}$ and $10^{\text {th }}$ clicks only. It is possible to observe a strong decrease in the initial click response (as already observed in Figure 27-G) and to further clicks. The comparison between control and mutants was not possible due to the strong amplitude decrease. For that, I measured spike synchrony, which, as expected, had a strong dependency on the rate of stimulation (Figure 29-C). There was a significant effect of frequency (ANOVA, $F(4,277)=76.71, p<0.0001$ ), and group (ANOVA, $F(1,277)=72.28, p<0.0001$ ) together with a significant interaction (ANOVA, $F(4$, $277)=21.4, p<0.0001$ ), but no effect of click number (ANOVA, $F(5,277)=0.16, p<0.97$ ). Suggesting that mutant animals had reduced spike synchrony. Individual frequency comparisons showed that the main effects were found below $14 \mathrm{~Hz}$ (ANOVA, $\mathrm{p}<0.0001$, $p<0.0001 p<0.0001$, for 5,8 and $10 \mathrm{~Hz}$ respectively). These results show that MBP ${ }^{e m x}$ mice present reduced rate coding at most of the rates that the $A C x$ is able to normally code, a result comparable to what is seen in $\mathrm{MBP}^{\text {shi }}$ mice but with a slightly milder effect. 
A

B

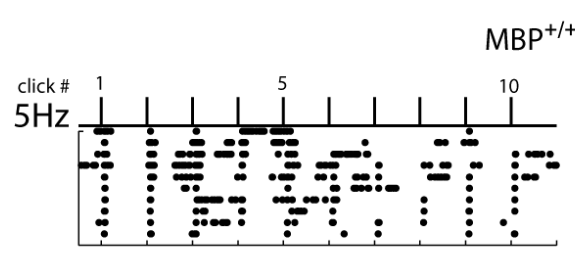

$5 \mathrm{~Hz}$

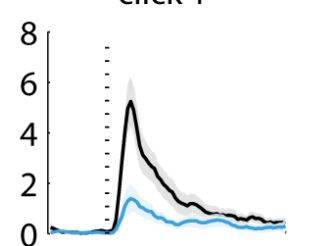

click 5

click 10

$\mathrm{Hz}$
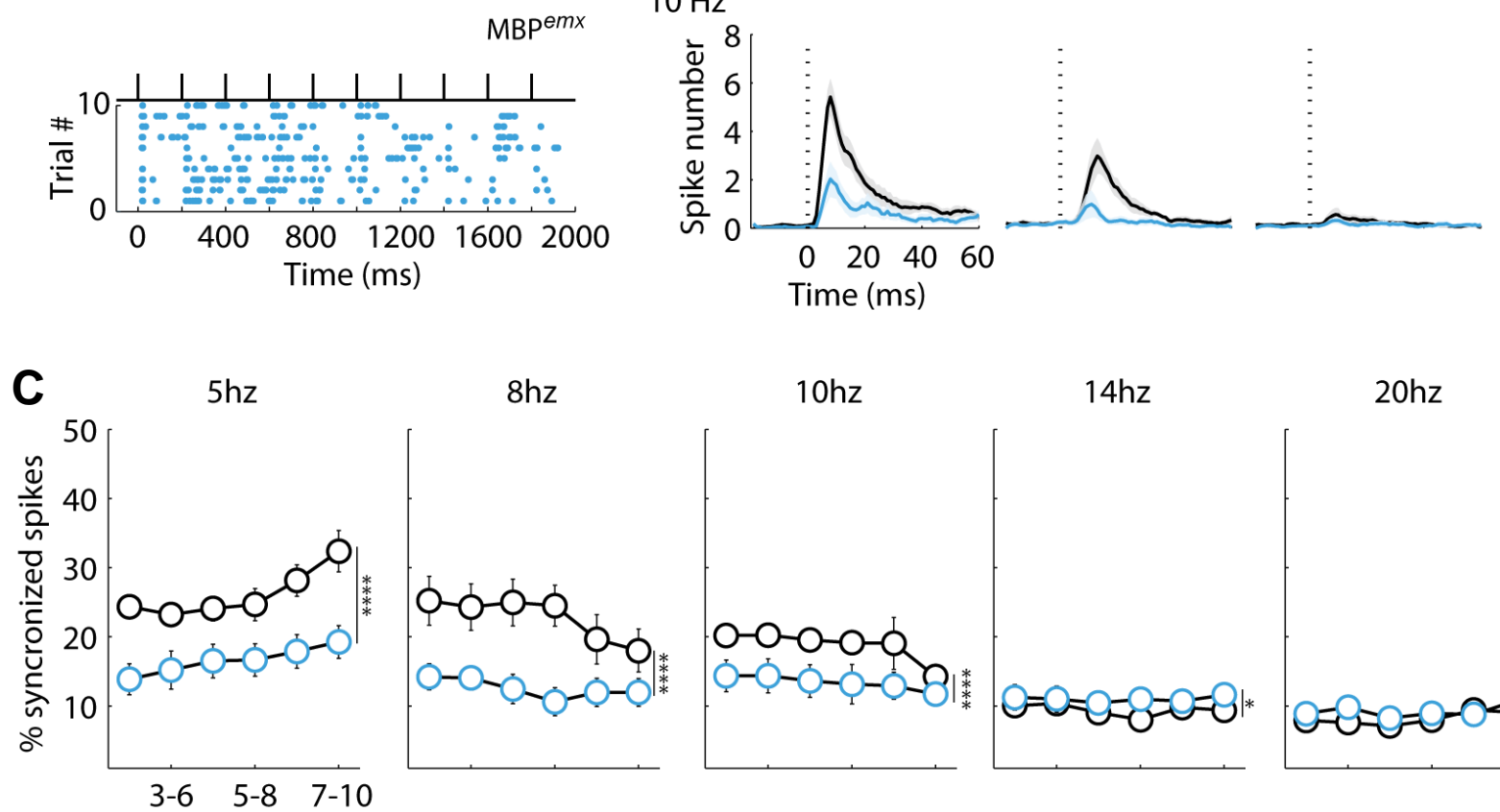

$8 \mathrm{hz}$

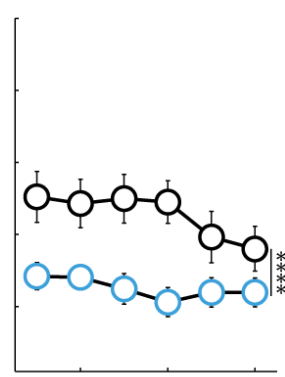

$10 \mathrm{hz}$

$14 \mathrm{hz}$

$20 h z$
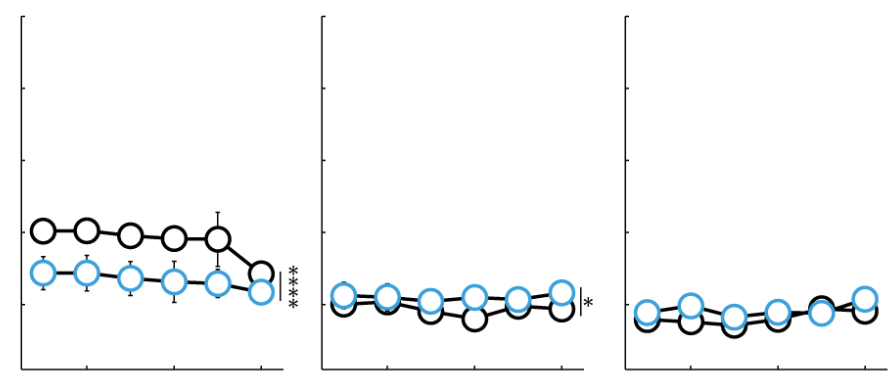

Sliding window

(click number)

Figure 29. Rate coding is affected in the auditory cortex of $\mathrm{MBP}^{\mathrm{emx}}$ mice.

A) An example raster plot of a control animal (upper, black) and a MBP ${ }^{e m x}$ mouse (lower, blue) in response to a set of 10 clicks depicted by the vertical bars above the raster plots. These examples correspond to the condition of $5 \mathrm{~Hz}$, in which each stimulus was $200 \mathrm{~ms}$ apart. Adaptation is observed, with lower spiking activity by the final clicks compared to the initial clicks. In B) we can see the mean PSTH of all animals recorded for control and mutant animals (conserving the same color-code). A blowup of click 1, 5 and 10 (as depicted in A) is shown. Thick lines show the mean PSTH of all animals recorded and the shaded area corresponds to the S.E.M. The upper panel shows the responses to a rate of $5 \mathrm{~Hz}$, while the lower to a rate of $10 \mathrm{~Hz}$. Vertical dotted lines represent stimulus onset. Responses mutant animals are strongly reduced in amplitude over all clicks. $n=6$ control and $n=6$ mutant animals. C) The quantification of spike synchrony confirmed that there is a significant difference between control and mutant mice (ANOVA, $\mathrm{p}<0.0001$ ) through the rates presented. Individual comparisons between groups considering all the clicks for each rate show that the strongest differences are at below $10 \mathrm{~Hz}$ (ANOVA, $p<0.0001, p<0.0001$, $p<0.0001, p=0.044$ and $p=0.06$ for rates from $5-20 \mathrm{~Hz}$ respectively). $n=5-6$ control animals and $n=4-6$ mutant animals. 


\subsubsection{Functional sensory consequences of a partial MBP loss in gray and white matter}

To understand the effects of a partial loss of MBP in the auditory system, I used the MBP ${ }^{\text {neo }}$ mice model. These animals have a loss of $\sim 80 \%$ in MBP at the protein level, and show a strong dysmyelination phenotype. Myelin is thinner and there are less axons myelinated (see Appendix Figure 49). In spite of the strong dysmyelination phenotype, these mice do not display the common behavioral abnormalities associated to a total dysmyelination, such as tremors or decreased life span, as is the case of the $\mathrm{MBP}^{\text {shi }}$ mice. For this reason the $\mathrm{MBP}^{\text {neo }}$ mice are a suitable model to study, behavioral consequences of a strong loss of myelin.

\section{Basic hearing properties}

To start with the auditory characterization of the $\mathrm{MBP}^{\text {neo }}$ model, and in collaboration with Nadine Herrmann and Dr. Nicola Strenzke, we did ABRs in these mice. ABRs measured in $\mathrm{MBP}^{\text {neo }}$ mice showed that mutant animals, like $\mathrm{MBP}^{\text {shi }}$, have a split wave II and a strong response delay starting from wave II on (Figure 30-A). I observed no differences at the threshold of sound detection which was at around $30 \mathrm{~dB}$ for control and mutant animals $(p=0.53)$ (Figure 30-B), which suggests that outer hair cell function is normal in these mice. In addition, there was a strong increase in response latencies that started from wave II and continued until wave $\mathrm{V}$ (Figure 30). C) (ANOVA, $p=0.74, p=0.013, p<0.0001, p<0.0001$, $\mathrm{p}=0.0001$ for waves I until $\mathrm{V}$ respectively). A decrease in response amplitude was also seen from wave II to IV (ANOVA, $p=0.18, p=0.057, p=0.009, p=0.0008, p=0.003$ for waves I to $V$ respectively). Overall these patterns were milder but very similar to what was observed in $\mathrm{MBP}^{\text {shi }}$ mice. This suggests that desynchronized responses due to partial dysmyelination are affecting responses in the brainstem. When quantifying the relative auditory gain, I observed no significant differences for the ratio of wave III/I $(p=0.083)$, but the strong reduction in the amplitude of wave IV in $\mathrm{MBP}^{\text {neo }}$ mice generated a significant reduction in the wave IV/I ratio $(p=0.0001)$ and a significant increase in wave $V / /$ ratio $(p=0.009)$ in $\mathrm{MBP}^{\text {neo }}$ mice. These results mean that at the latter auditory brainstem station (i.e. inferior colliculus) there is a gain increase in $\mathrm{MBP}^{\text {neo }}$ mice, probably as a compensatory mechanism for the loss of wave II-III stability. The gain increase for wave $V$ was $\sim 100 \%$, this suggests similar mechanisms of gain increase between $\mathrm{MBP}^{\text {neo }}$ and $\mathrm{MBP}^{\text {shi }}$ mice and a proportional relationship between the amount of gain and the level of dysmyelination. 
A

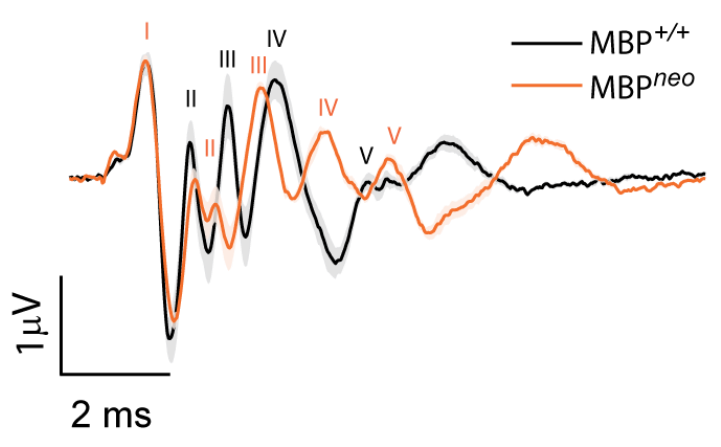

B

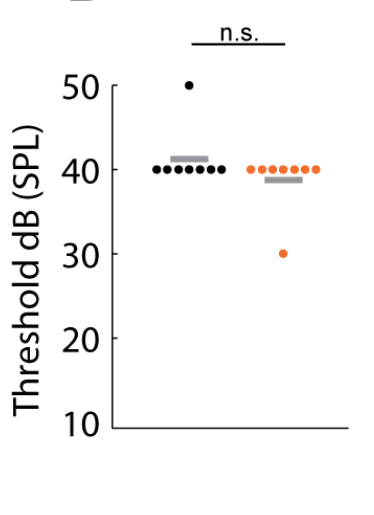

C
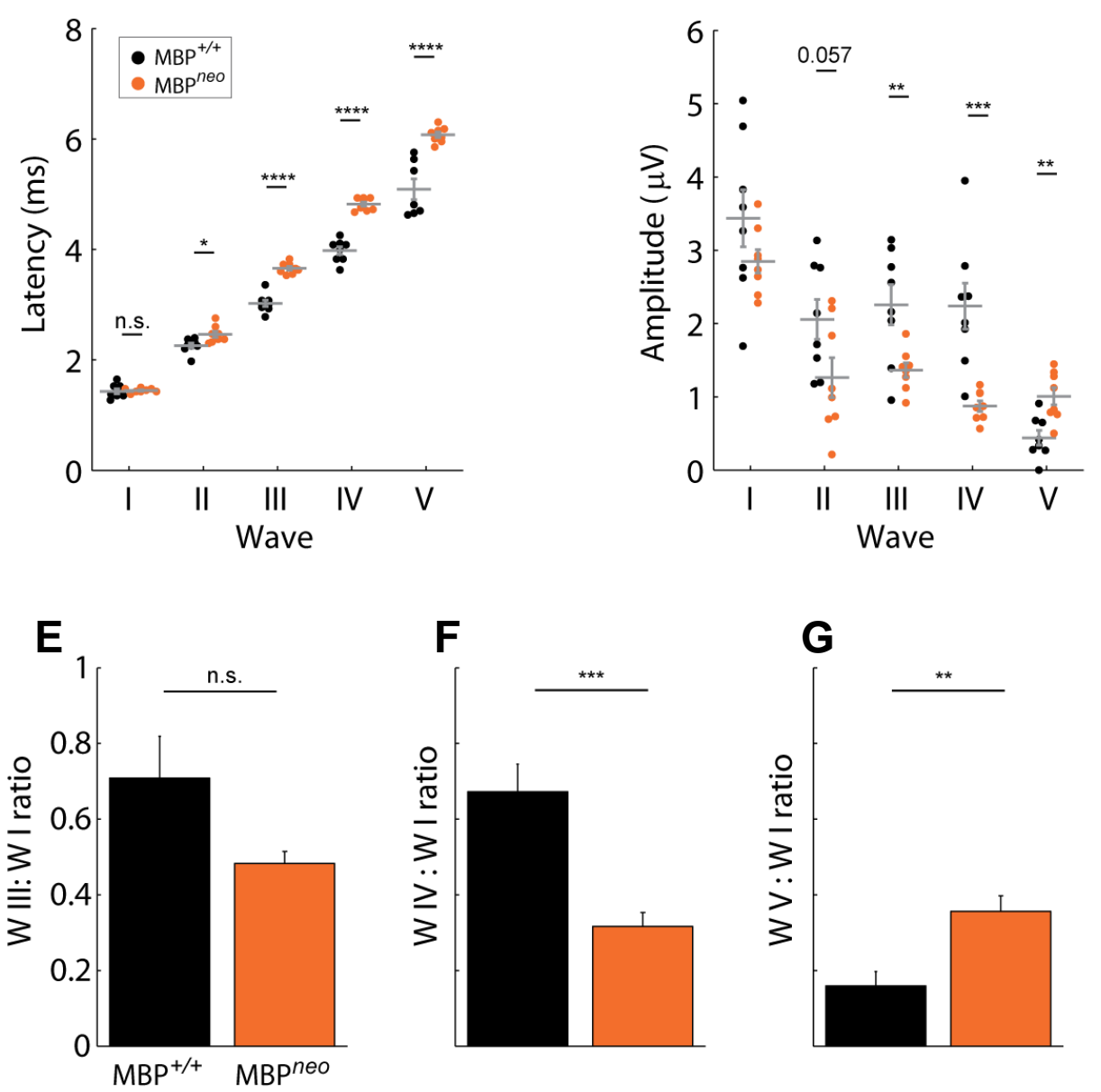

Figure 30. Subcortical auditory processing is impaired $\mathrm{MBP}^{\text {neo }}$ mice.

A) The 5 characteristic peaks of ABRs, belonging to 5 different auditory stations, from the auditory nerve (I) untill the inferior colliculus (V) for control (black) and $\mathrm{MBP}^{\text {neo }}$ (orange) animals. $\mathrm{MBP}^{\text {neo }}$ (orange) show strong delays in auditory potentials starting from wave II, elicited by clicks at $80 \mathrm{~dB}$. In addition, the shape of wave II is split. B) No differences were seen in hearing thresholds $(p=0.53)$, and indirect measure of outer hair cell function. Gray bars show the mean per group. C) Waves II to $\mathrm{V}$ are delayed in $\mathrm{MBP}^{\text {neo }}$ animals, compared to control. D) $\mathrm{MBP}^{\text {neo }}$ mice have a reduction in wave amplitude that starts from wave III until wave IV $(p=0.0080$ and $p=0.0008)$. Grey bars show the mean and S.E.M. per group. E) No changes in central auditory gain were observed for wave III $(p=0.083)$. A significant decrease in central auditory gain was seen for $F)$ wave IV $(p=0.0001)$ and a significant increase in gain was seen for $G)$ wave $V(p=0.009)$, meaning that compensatory mechanisms take place in the latest brainstem station, probably due to an amplitude difference in earlier brainstem stations. Plot shows the mean + S.E.M. $n=8$ control and $n=8$ mutant animals. 


\section{Basic hearing properties}

By doing extracellular MUA recordings, a more specific readout of the changes in neuronal firing of a small population of neurons is achieved. I then tested MUA activity in further auditory stations, such as the $\mathrm{ACx}$ and the $\mathrm{IC}$. In response to a click stimulus, $\mathrm{MBP}^{\text {neo }}$ mice show delayed responses in both ACx and IC (Figure 31-A and D). In both, the ACx and the $I C$, there was a significant increase in latencies $(p=0.0003$ and $p=0.019$ respectively). Nevertheless, the increase was much larger in the ACx than in the IC $(6.05 \mathrm{~ms}$ vs $3.12 \mathrm{~ms}$
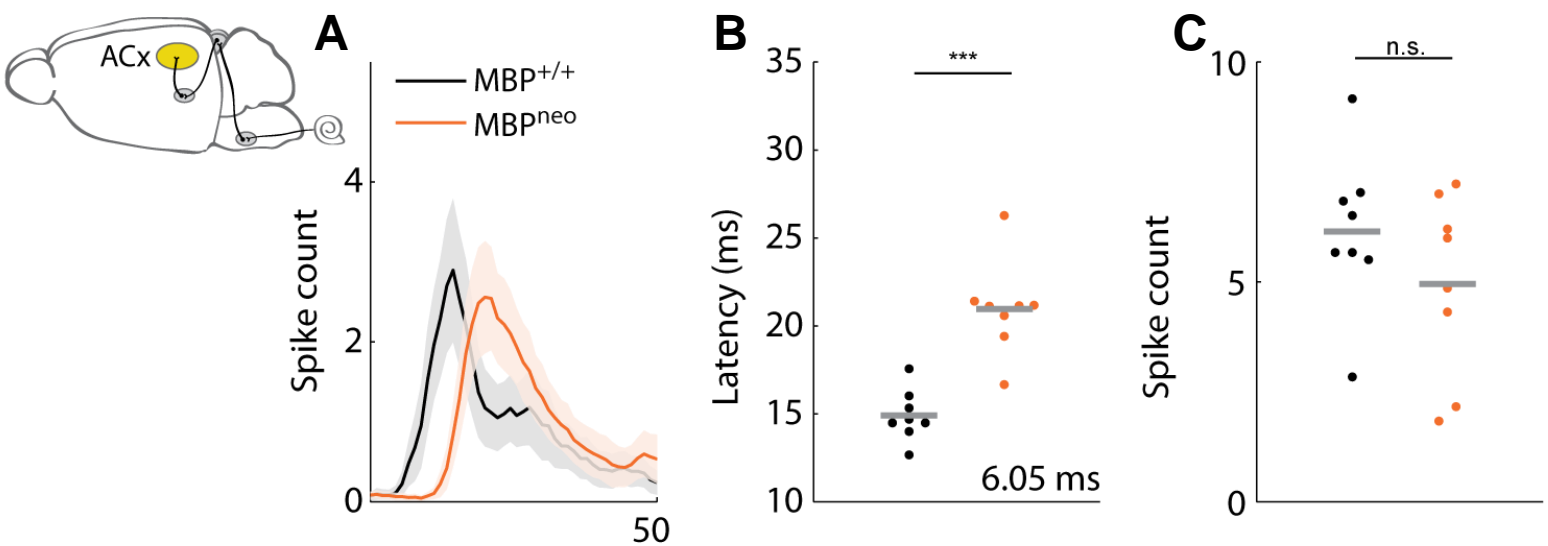

Time (ms)

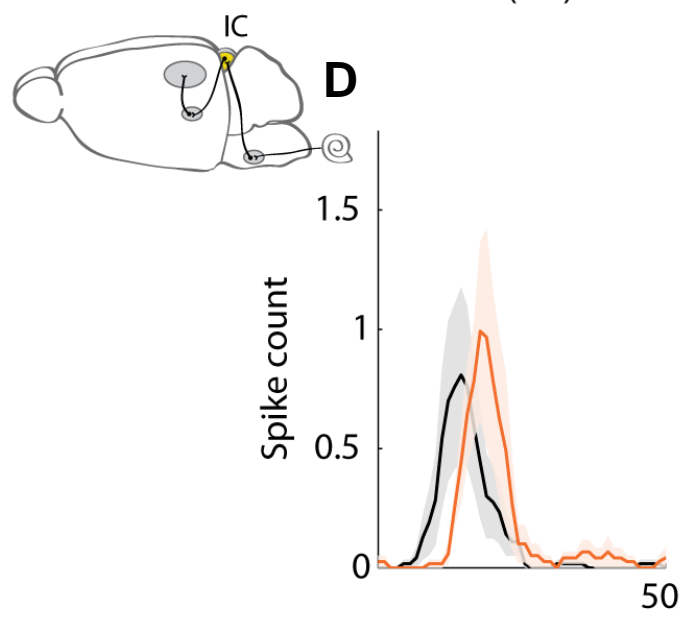

E

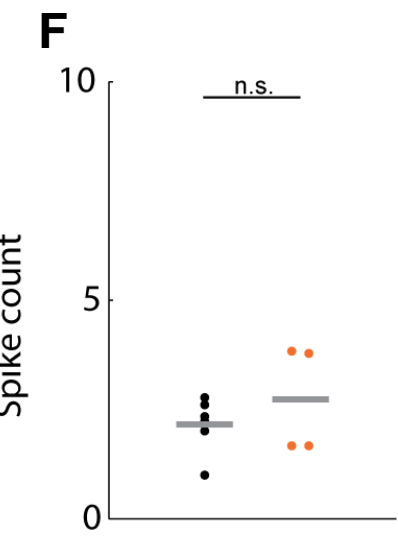

Time (ms)

Figure 31. Auditory cortex and inferior colliculus responses are impaired in $\mathrm{MBP}^{\text {neo }}$ mice.

A) PSTH of the auditory cortex response to a click stimulus for control (black) and $\mathrm{MBP}^{\text {neo }}$ (orange) mice. A strong increase in response latency is seen at this level. Upon quantification, in B) is possible to observe a strong latency increase of control (black dots) compared to mutant (orange dots) animals ( $p=0.0003)$; the number in the right lower corner depicts the difference between control and mutant means. Grey bars depict the mean per group. C) No significant differences are seen in response amplitude in the ACx $(p=0.36)$. D) Same as in A) but for the inferior colliculus. Latency shift is not so strong in the inferior colliculus compared to the cortex. E) Latency quantification shows a significant response delay in the IC $(p=0.019)$, nevertheless, the differences between the means is almost half of what is seen in the ACx (3.12 ms vs $6.05 \mathrm{~ms}$ ). F) No significant differences are seen in response amplitude in the IC $(p=0.71)$. ACx $n=8$ mutant mice and $n=8-9$ control mice. IC $n=4$ mutant mice and $n=6$ control mice. 
increase respectively). This suggests there was a cumulative effect of the latency differences across the auditory pathway that accounts for having longer delays in later auditory stations. In addition, there were no significant differences in response amplitudes neither in the $A C x$, nor in the IC ( $p=0.36$ and $p=0.71$ respectively). Similar effects of this model were seen in the $\mathrm{MBP}^{\text {shi }}$, nevertheless the latter had an overall stronger auditory phenotype. Since I observed milder abnormalities with the $\mathrm{MPB}^{\text {neo }}$, I expect that the mechanisms underlying the loss of desynchronization, or the gain increase are at least partially shared.

\section{A comparison with white matter tracts}

Once the characterization at the gray matter level was done, my interest was to understand the functional impact in white matter, of partial myelin loss using the $\mathrm{MBP}^{\text {neo }}$ mice. For this purpose, I collaborated with Andrea Trevisiol, who did optic nerve recordings in this model. $\mathrm{MBP}^{\text {neo }}$ mice, as seen in Figure 32-A, have a triphasic CAP, such as the one seen in control animals. Nevertheless, a strong decrease in amplitude and the appearance of a hyperpolarizing phase is seen, similar to what we observed with the MBP ${ }^{\text {shi }}$ mice. I measured the percentage of conduction velocity change with respect to the control animals and observed a significant reduction in conduction velocity $(C V)$ in MBP ${ }^{\text {neo }}$ mice $(p<0.0001)$. $\mathrm{MBP}^{\text {neo }} \mathrm{ON} \mathrm{AP}$ is delayed about $0.6 \mathrm{~ms}$ from the control peak, with a CV decrease of approximately $28 \%$ in the optic nerve (Figure $32-\mathrm{B}$ ). When comparing this model to the $\mathrm{MBP}^{\text {shi }}$ mice, I saw that $\mathrm{MBP}^{\text {neo }}$ mice also had a significant reduction in CV compared to $\mathrm{MBP}^{\text {neo }}(\mathrm{p}=0.00038)$, suggesting a strong correlation between the amount of dysmyelination and the percentage of reduction in $\mathrm{CV}$. For better comparison between the $\mathrm{MBP}^{\text {neo }}$ and the $\mathrm{MBP}^{\text {shi }}$ model, I did a correlation between the degrees of dysmyelination (\% of MBP reduction at the protein level) with the amount of CV decrease. The fit of this correlation corresponds to a second order polynomial equation. This correlation shows that, in white matter, the dependence of conduction velocity decrease with dysmyelination follows an exponential fashion (Figure $32-\mathrm{C}$ ). $\mathrm{MBP}^{\text {neo }} \mathrm{ON}$ do not show a significant decrease in excitability (Figure 32-D), as observed in the MBP ${ }^{\text {shi }} \mathrm{CAP}$. There was a significant effect of stimulation frequency in the normalized CAP amplitude (ANOVA, $F(9,110)=1141.41, p<0.0001$ ), and a strong effect of group (ANOVA, $F(1,110)=19.35, p<0.0001$ ) without an interaction (ANOVA, $F(9,110)=1.81$, $p<0.075)$, suggesting no differences in excitability threshold. $\mathrm{MBP}^{\text {neo }} \mathrm{CAP}$ has a strong reduction in response amplitude. There was a significant effect of stimulation frequency in the depolarization CAP area (ANOVA, $F(9,110)=86.69, p<0.0001$ ), and a strong effect of group (ANOVA, $F(1,110)=165.5, p<0.0001$ ) with an interaction (ANOVA, $F(9,110)=8.51, p<0.0001$ ). 
The decrease in CAP area is smaller when compared to the MBP ${ }^{\text {shi }}$ (ANOVA, $p<0.0001$ ) and the hyperpolarizing phase is significantly different than control animals. There was also a significant effect of the stimulation strength in the hyperpolarization CAP area (ANOVA, $F(9,110)=41.24, p<0.0001$ ), and a strong effect of group (ANOVA, $F(1,110)=676.66$, $p<0.0001$ ) with an interaction (ANOVA, $F(9,110)=42.39, p<0.0001$ ). In addition, the hyperpolarizing area was smaller than the MBPshi one (ANOVA, $p<0.0001$ ). Interestingly, the $\mathrm{MBP}^{\text {neo }}$ potentials seem to have an intermediate phenotype between the control and $\mathrm{MBP}^{\text {shi }}$ ON. 
A

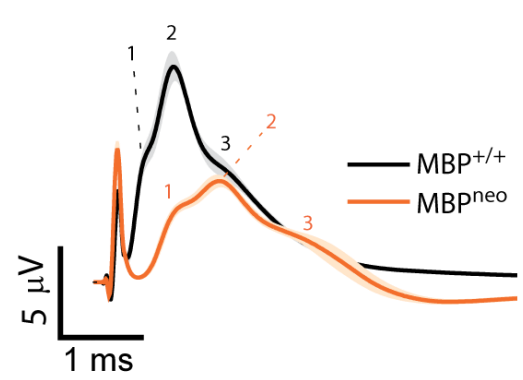

B

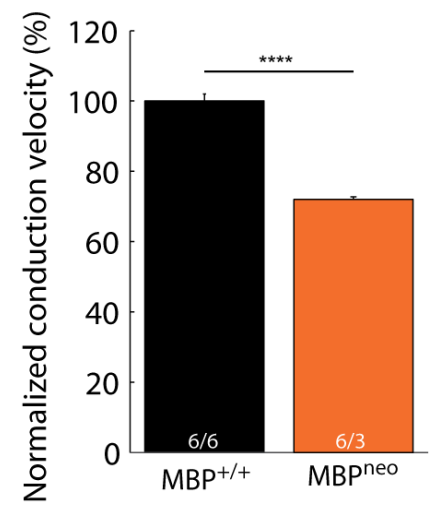

$\mathbf{E}$

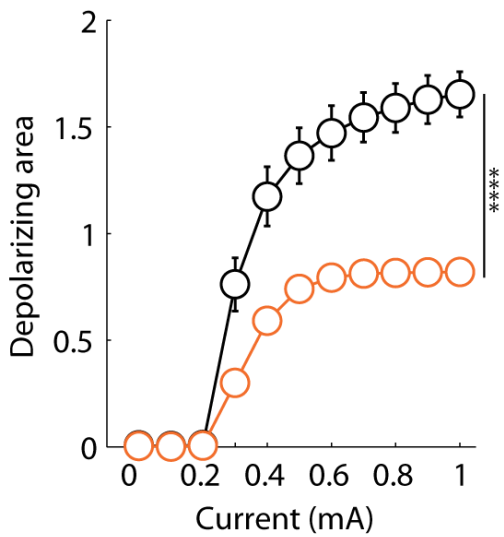

C

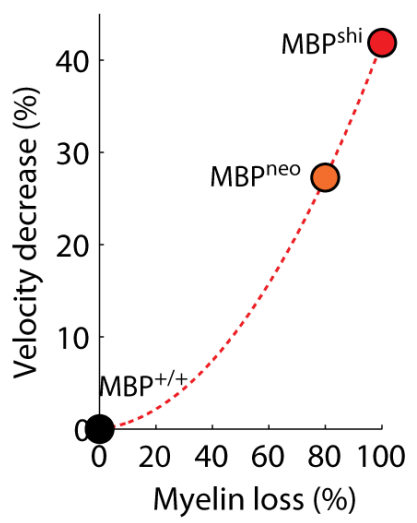

$\mathbf{F}$

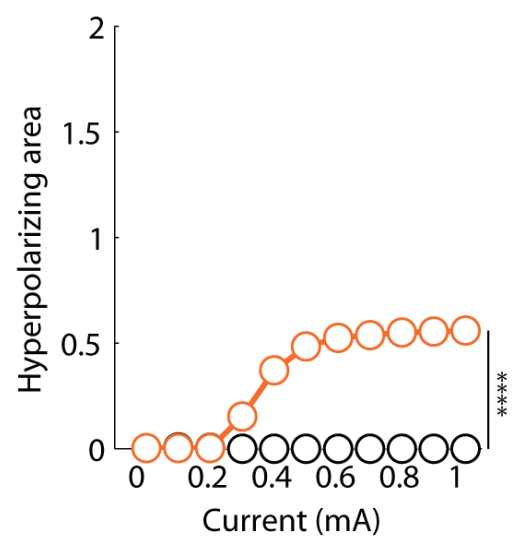

Figure 32. White matter abnormalities that arise from $\mathrm{MBP}^{\text {neo }}$ mutation.

A) Compound action potentials from the optic nerve of $\mathrm{MBP}^{\text {neo }}$ mice (orange) and control (black) at a stimulation intensity of $1 \mathrm{~mA}$, traces show the mean of all optic nerves pulled together; shadow around the mean shows the S.E.M. The typical triphasic CAP seen in control animals maintains its shape in $\mathrm{MBP}^{\text {neo }}$ mice, but there is a strong reduction of the response amplitude, accompanied by a mild hyperpolarizing phase. B) Conduction velocity was measured at an intensity of $0.7 \mathrm{~mA}$ stimulation $\mathrm{MBP}^{\text {neo }}$ mice show a strong reduction in conduction velocity $(p<0.0001)$. In addition, there is also a difference between conduction velocity decrease in MBP ${ }^{\text {neo }}$ mice compared to $\mathrm{MBP}^{\text {shi }}(\mathrm{p}=0.00038)$. C) A graph for comparison of the velocity reduction of $\mathrm{MBP}^{\text {neo }}$ to $\mathrm{MBP}^{\text {shi }}$ mice. A second degree polynomial fit was applied. D) Excitability of $\mathrm{MBP}^{\text {neo }}$ is suboptimal, showing a significant difference in the amount of current is needed for the activity of shiverer axons $(p<0.0001)$. Higher stimulation thresholds are required to activate $\mathrm{MBP}^{\text {neo }}$ axons. E) Significant decrease in the depolarizing area $(\mathrm{p}<0.0001)$ of $\mathrm{MBP}^{\text {neo }}$ and $\left.\mathrm{F}\right)$ significant increase in the hyperpolarizing phase $(p<0.0001)$ of $\mathrm{MBP}^{\text {neo }}$ that is not present in the control animals. $n=8$ nerves from 7 control animals and $n=6$ nerves from $3 \mathrm{MBP}^{n e o}$ animals. All measures show the mean and error bars the S.E.M. 


\subsubsection{Mice with partial myelin loss have subtle impairments in temporal acuity and rate coding in the auditory cortex}

Is temporal acuity affected with partial dysmyelination?

To understand the effects that a partial loss of myelin have on temporal acuity and rate coding in the auditory cortex, I used the same sound paradigms described in section 1.2.3. $\mathrm{MBP}^{n e o}$ mice presented a mild impairment of temporal acuity in the ACx. In Figure 33-A, we can see an individual example of a control and an $\mathrm{MBP}^{\text {neo }}$ recording where one can appreciate the loss of spiking activity in the $\mathrm{MBP}^{\text {neo }}$ mice to the post-gap sound. The post-gap PSTH at different gap lengths, in Figure 33-C, show that pre-gap responses are not significantly different between groups, amplitude wise (ANOVA, $F(1,273)=0.27, p=0.60$ ). This allowed making a comparison between control and mutant animals, taking the post-gap window at the different selected gaps. It can be seen clearly, that $\mathrm{MBP}^{\text {neo }}$ mice do not respond to gaps below $3 \mathrm{~ms}$, and after, the responses are significantly smaller compared to control animals. Overall, I saw a significant effect of gap (ANOVA, $F(5,1738)=16.71$, $p<0.0001$ ), and a strong effect of group (ANOVA, $F(1,1738)=79.7, p<0.0001$ ), but no interaction (ANOVA, $F(5,1738)=1.27, p=0.27$ ). Individual comparisons at each gap showed that the significant difference between groups was decreasing with the increase in gap (ANOVAS $p<0.0001, p<0.0001, p=0.0015, p=0.19, p=0.01, p=0.003$ for gaps from $0.5 m s$ to 5 $\mathrm{ms}$ respectively), indicating that, for example at $3 \mathrm{~ms}$ gap, $\mathrm{MBP}^{\text {neo }}$ mice responses were not significantly different from control animals. This suggests that the mild impairment in gap detection seen in these animals might be restricted to very short gaps, lower than $3 \mathrm{~ms}$. When measuring the internal spiking probability of each recording file, as expected, there was a strong correlation between the gap length and a reduction of the $p$-value, meaning that longer gaps, elicited stronger post-gap responses, which was reflected in a lower p-value. In Figure 33-B, we can clearly see this trend for both control and mutant animals. I observed a significant effect of gap overall (Kruskal-Wallis test, $\left.x^{2}(9, n=347)=27.03, p=0.0014\right)$. Nevertheless, no significant effect of group was observed (Kruskal-Wallis test, $x^{2}(1$, $\mathrm{n}=347)=0.81, \mathrm{p}=0.36$ ), and no differences in baseline activity were seen between control and mutant animals $(p=0.13)$. These results suggest that $\mathrm{MBP}^{\text {neo }}$ mice tend to have a slight reduction in spiking probability after the presentation of very small gaps, nevertheless, these small responses are still significantly different from the baseline activity in a similar way to what it is observed for control animals. This reflects a mild impairment in responding to the post-gap sound in $\mathrm{MBP}^{\text {neo }}$ mice. 
A
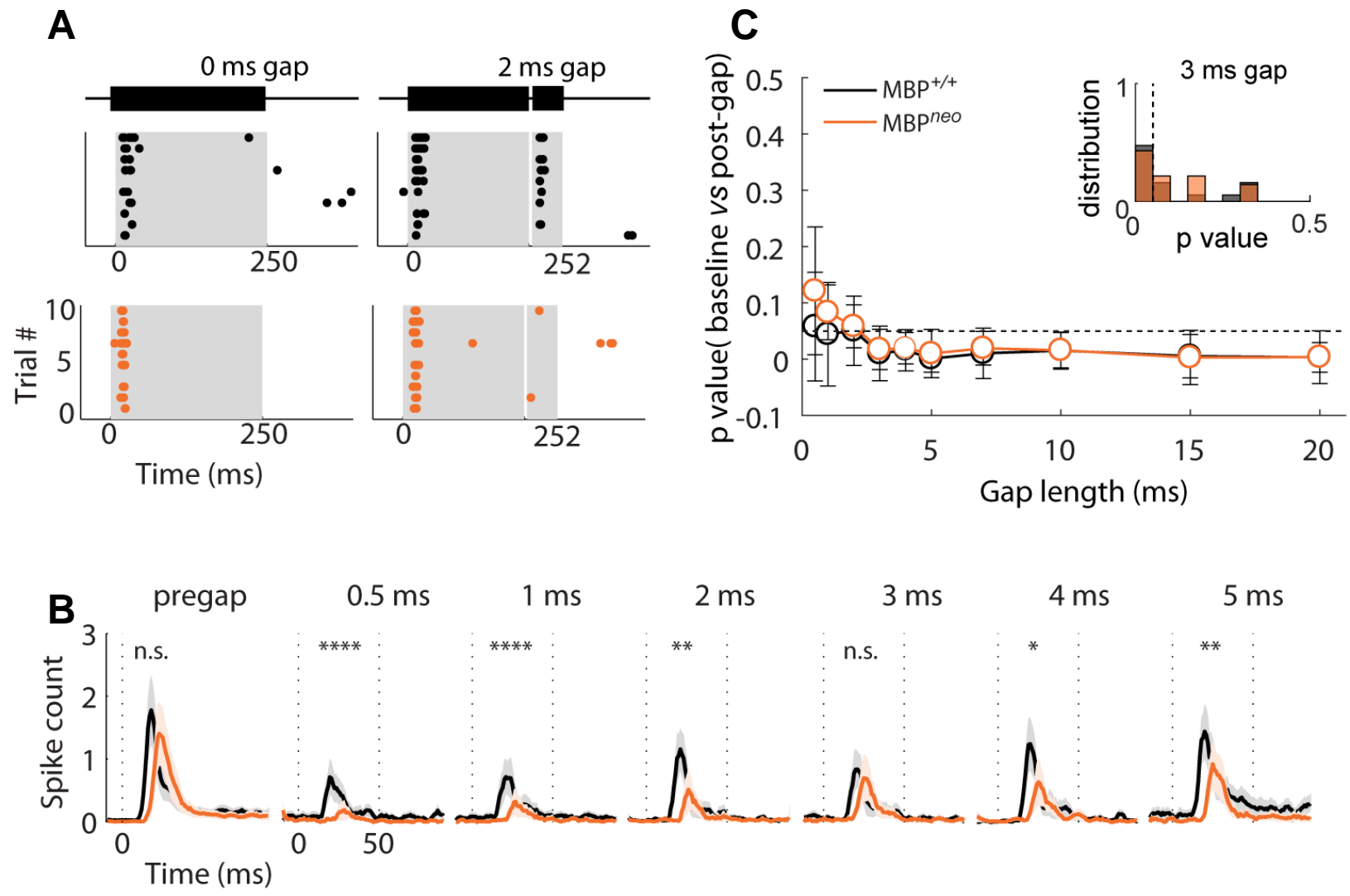

Figure 33. Impaired temporal acuity in $\mathrm{MBP}^{\text {neo }}$ mice.

A) An individual example of the gap-detection recordings of a control (upper, black) and mutant (lower, orange) mouse. These raster plots represent the spiking activity (individual dots) that occurs during sound presentation (gray patches) to the 10 stimulus repetitions. Above the raster plots, a representation of the sounds played is presented in black bars, for a sound without a gap (0 ms gap, left side) and a sound with a $2 \mathrm{~ms}$ gap (right side). B) The average PSTH of all animals for control (black) and mutant (orange) groups with the S.E.M represented as a shaded are following the same color code. Pre-gap and post-gap responses to the presentation of 0.5 to $5 \mathrm{~ms}$ are represented. Significant differences were seen between groups for the PSTH comparison (ANOVA, $p<0.0001$ ). $n=7$ control and $n=8$ mutant mice. C) Quantification of the differences between baseline and post-gap activity (50 ms window). A dotted black line depicts the threshold of $p=0.05$ for significance. The longer the gap, the more significance values fall below 0.05 for both groups. A significant difference for gaps was observed (Kruskal-Wallis test, $p=0.0014$ ) but no group difference (Kruskal-Wallis, $\mathrm{p}=0.36$ ). The plot shows the median values for both groups, error bars show the standard error of the median. The internal distribution plot in $\mathrm{C}$ ), shows all the $\mathrm{p}$-value distributions for the $3 \mathrm{~ms}$ gap condition in control (gray) and mutant (orange) mice. A vertical black dotted line depicts the $p$ value corresponding to 0.05 . It is possible to see that both the control and mutant responses to the $3 \mathrm{~ms}$ gap are significantly different than baseline activity (left side of the vertical line) and have very similar distributions. $n=17-22$ recording sites of 7 control animals and $\mathrm{n}=13-17$ recordings of $8 \mathrm{MBP}^{\text {neo }}$ mice.

\section{Is rate coding affected with partial dysmyelination?}

Rate coding was assessed in $\mathrm{MBP}^{\text {neo }}$ mice. In Figure 34-A, we can observe an example raster-plot of a $\mathrm{MBP}^{+/ t}$ mouse and a $\mathrm{MBP}^{\text {neo }}$ mouse to a set of 10 clicks at a rate of $5 \mathrm{~Hz}$. In this individual animal example, $\mathrm{MBP}^{\text {neo }}$ mouse shows more variability in the spiking activity 
from clicks two to 5 as compared to the control example. Figure 34-B shows the mean of all recorded animals for the control group and the $\mathrm{MBP}^{\text {neo }}$ mice, representing a blowup of only the $1 \mathrm{st}, 5$ th and $10^{\text {th }}$ click. The initial click response is not different in amplitude, nevertheless, $\mathrm{MBP}^{\text {neo }}$ responses seem smaller than control ones at click 5 for the condition of 5 and $10 \mathrm{~Hz}$ (upper and lower panel, middle graph Figure 34-B). The percentage of spike synchrony had a significant correlation with the rate of stimulus presentation (ANOVA, $F(4,268)=37.64$, $p<0.0001$ ), and there was a significant effect of group (ANOVA, $F(1,268)=8.16, p=0.0046$ ) together with a significant interaction (ANOVA, $F(4,268)=5.33, p<0.0004$ ), but no effect of click number (ANOVA, $F(5,268)=0.5, p<0.77$ ). Individual frequency comparisons showed that the main effects were found at 5 and $8 \mathrm{~Hz}$ (ANOVA, $\mathrm{p}=0.0014$ and $\mathrm{p}=0.0015$ respectively). These results show that $\mathrm{MBP}^{\text {neo }}$ mice present reduced rate coding in the $\mathrm{ACx}$, a result comparable to what is seen in $\mathrm{MBP}^{s h i}$ mice but with a milder effect. 
A

B
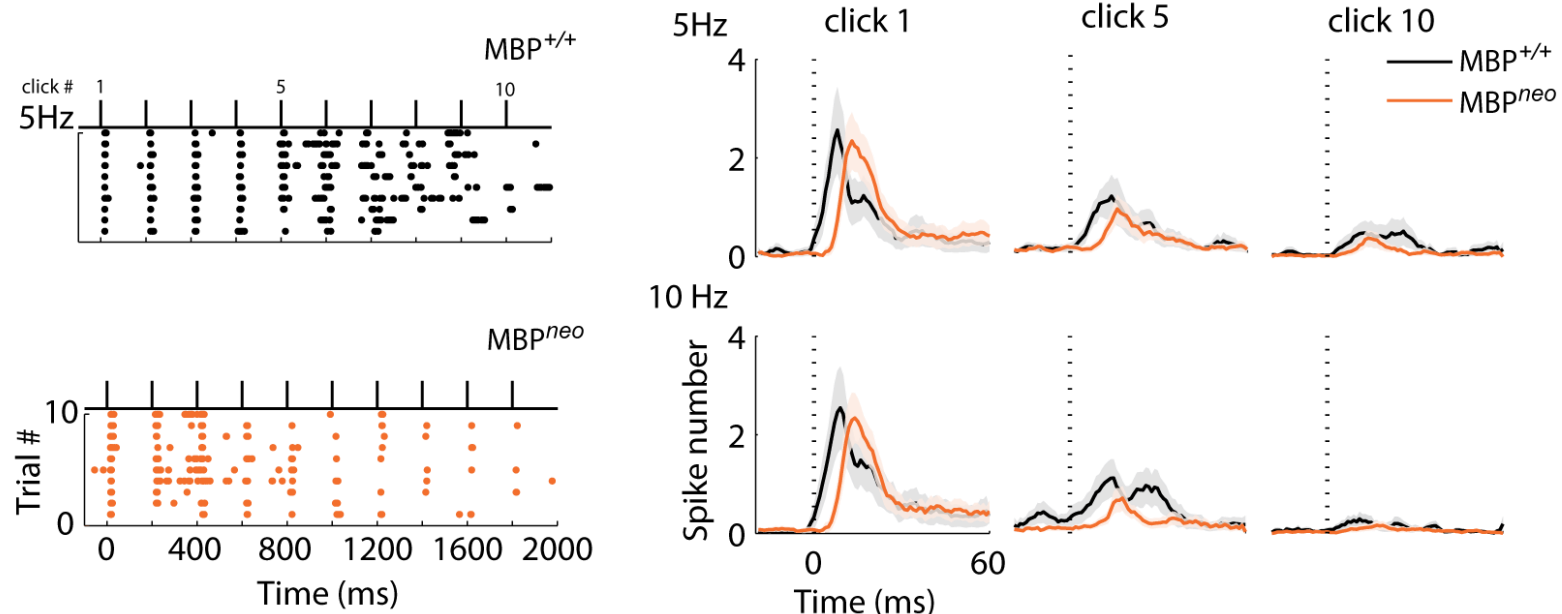

$10 \mathrm{~Hz}$
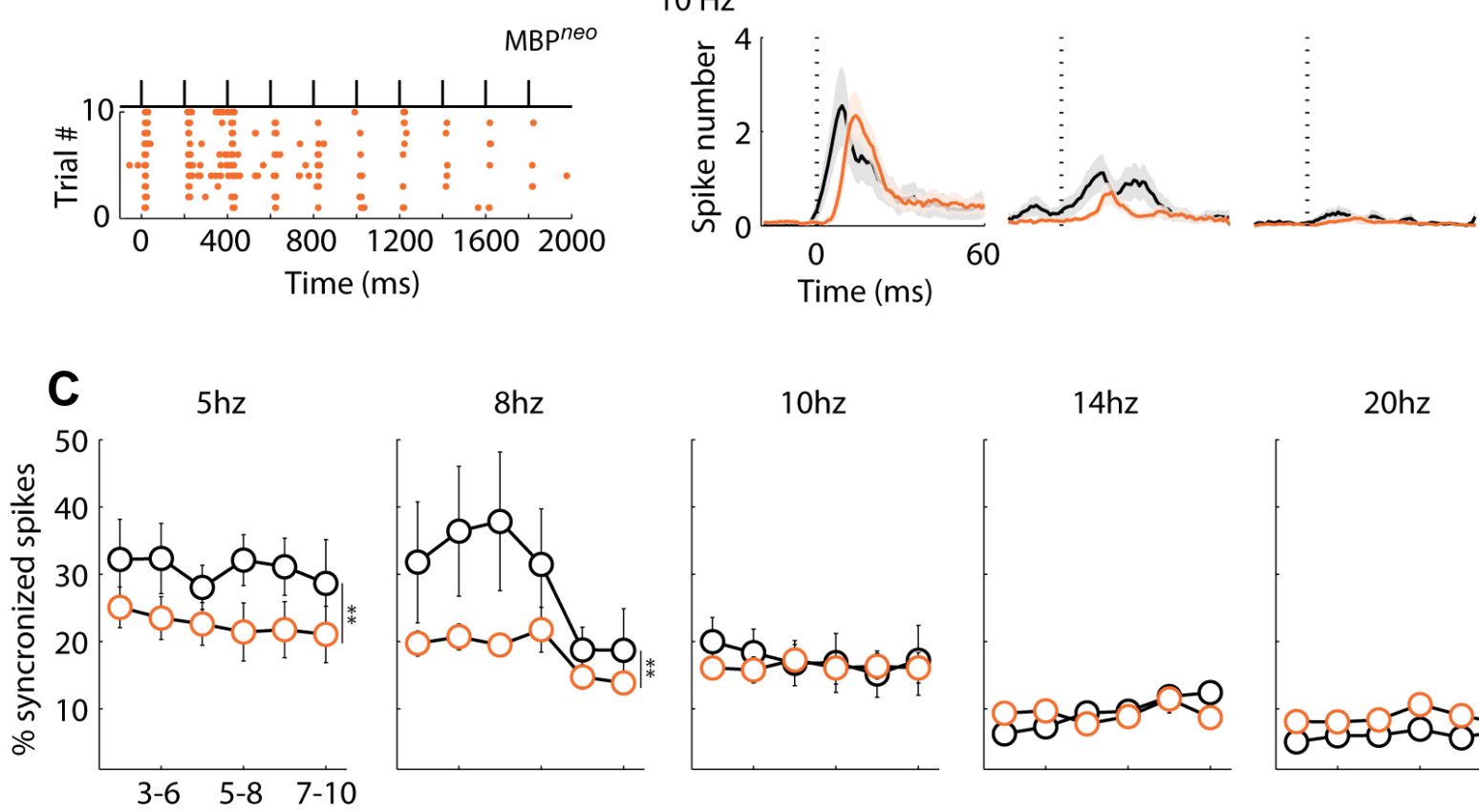

$8 \mathrm{hz}$

$10 \mathrm{hz}$

$14 \mathrm{hz}$

$20 \mathrm{hz}$
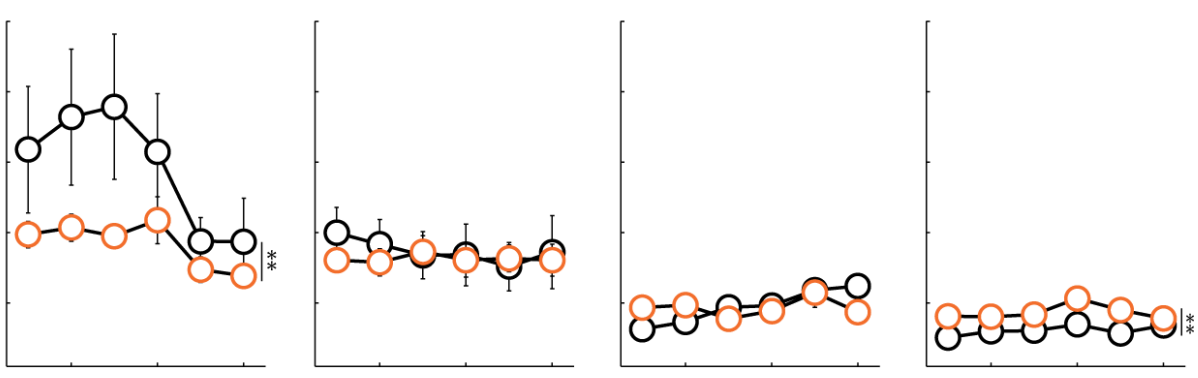

Sliding window

(click number)

Figure 34. Rate coding is slightly affected in the auditory cortex of $\mathrm{MBP}^{\text {neo }}$ mice.

A) An example raster plot of a control animal (upper, black) and an $\mathrm{MBP}^{\text {neo }}$ mouse (lower, orange) in response to a set of 10 clicks depicted by the vertical bars above the raster plots. These examples correspond to the condition of 5 $\mathrm{Hz}$, in which each stimulus was $200 \mathrm{~ms}$ apart Adaptation is observed, with lower spiking activity by the final clicks compared to the initial clicks. In B) we can see the mean PSTH of all animals recorded for control and mutant groups (conserving the same color-code). A blowup of click 1, 5 and 10 (as depicted in A) is shown. Thick lines show the mean of all animals recorded and the shaded area corresponds to the S.E.M. The upper panel shows the responses to a rate of $5 \mathrm{~Hz}$ while the lower to a rate of $10 \mathrm{~Hz}$. Vertical dotted lines represent stimulus onset. Responses in control and mutant animals are similar in amplitude to the first click. A slight reduction of response amplitude is seen in MBP ${ }^{\text {neo }}$ mice with increasing clicks. $n=8$ control and $n=8$ mutant animals. C) The quantification of spike synchrony showed that there is a significant difference between control and mutant mice (ANOVA, $p=0.0046$ ) through the frequencies presented. Individual comparisons between groups considering all the clicks for each rate show that the strongest differences are at 8 and $10 \mathrm{~Hz}$ (ANOVA, $p=0.0014, p=0.0015, p=51, p=0.87$ and $p=0.0018$ for rates from 5$20 \mathrm{~Hz}$ respectively). $n=3-8$ control animals and $n=5-8$ mutant animals. 


\subsubsection{Partial myelin loss impairs temporal acuity at the behavioral level}

Perhaps one of the most interesting questions when studying the neurophysiology of an organism is how specific circuit defects have an impact in behavior. After characterizing the type of abnormalities that mice with a reduction in myelin have, in the ACx, my question was if these neuronal defects actually had a behavioral correlate and if some of the auditory defects that are seen in patients with myelin abnormalities would be correlated to any of the processing problems I observed. Since using the $\mathrm{MBP}^{\text {shi }}$ mice for behavioral tasks presents a strong challenge, due to the reduced life span of the animals and their motor abnormalities, I decided to test the $\mathrm{MBP}^{\text {neo }}$ mice, which have shown milder impairments in temporal acuity and rate coding. For this matter, I used two behavioral approaches, one that is a spontaneous, reflex-like response (section 1.2.8.1) and gap detection using a long-term learning paradigm in the AudioBox (section II.IX.II).

\subsubsection{Gap-elicited pre-pulse inhibition of the acoustic startle reflex in $M_{B} P^{\text {neo }}$ mice}

Since I observed that the electrophysiological gap detection paradigm is sensitive to differences in myelination levels, I was interested in implementing a behavioral correlate of the electrophysiological measure of gap detection, to understand how temporal acuity defects in the cortex affect sound perception. For this, I used gap-pre-pulse inhibition of the acoustic startle reflex, a paradigm that is widely used for assessing the detection of gaps at the behavioral level in different animal models (Dehmel et al., 2012; Friedman et al., 2004; Moreno-Paublete et al., 2017).

Pre-pulse inhibition of the ASR is a mechanism of sensorimotor gating. The acoustic startle response (ASR) is a fast motor response, observed in all mammals, to an intense sudden sound stimulus. This usually includes contraction of facial and body muscles and eye closure, heart rate acceleration and pause of ongoing behaviors (Koch, 1999). When another non-startling sound (pre-pulse) is presented just before the starling sound, the startling reflex is partially inhibited in a process known as the pre-pulse inhibition (PPI) of the startle reflex, which is the quantitative measure of sensorimotor gating. In the CNS, this neural process helps the filtering of sensory information to help the processing at higher order cortical brain areas (Koch, 1999). Sensorimotor gating is affected in schizophrenic patients (Engel et al., 
2015; Haß et al., 2017; Mena et al., 2016), and in general, a variety of neuropsychological pathologies have been associated with a reduced ability of gaiting information (for review, see (Koch, 1999). This reflex probably has an important behavioral advantage to prepare and protect an animal from an aversive stimulus. This behavior can be modulated (either enhanced of attenuated) by a series of external and internal aspects, thereby it provides a reliable measure of individual sensorimotor gating. In mammals, the ASR is believed to be mediated by the brainstem. It has been proposed that the ASR circuit relays in three interconnected structures: the cochlear nucleus, the neurons in the caudal pontine reticular formation and cranial and spinal motor neurons (Koch, 1999; Lee et al., 1996; Yeomans and Frankland, 1995). Silent gaps appearing in a continuous background, just before the startling sound, can also work as a pre-pulse and induce PPI of the ASR. The gap-elicited inhibition of the ASR has been widely used for testing temporal acuity in rodent animal models (Clark et al., 2000; Dehmel et al., 2012; Moreno-Paublete et al., 2017; Popelář et al., 2017; Walton et al., 1997). Measurements were performed as explained in Materials and Methods (section II.IX.I).

In Figure 35-A one can observe a brief scheme of the sounds played for this task. A background sound that consisted of a $70 \mathrm{~dB}$ (SPL) broad band noise was followed by gaps of different lengths (e.g. 5 or $25 \mathrm{~ms}$ ). After each gap, a period of $50 \mathrm{~ms}$ of the background BBN was played and then followed by a 40 ms startle noise at $\sim 105 \mathrm{~dB}$. In Figure 35-B, consistent with previous reports, that there was a strong effect of the length of a silent gap placed before the startle pulse. Short gaps were more difficult to be detected and elicited less inhibition, compared to longer gaps (Popelář et al., 2017). Independent controls were used for each mutant line and measured together to reduce variability of the testing day. Nevertheless, since there were no differences between all the control animals of the different lines tested, I pulled together all controls of the $\operatorname{MBP}^{\text {neo }}(\mathrm{n}=7), \operatorname{MBP}^{\text {shi }}(\mathrm{n}=2)$, and $\operatorname{MBP}^{e m x}(\mathrm{n}=6)$ lines (ANOVA, $F(1,63)=1.7, p<0.19 ; F(1,99)=0.2, p<0.65 ; F(1,54)=2.16, p<0.14$, respectively). $\mathrm{MBP}^{\text {neo }}$ mice show a significant decrease in ASR inhibition in the presence of different gap lengths when compared to control animals. An ANOVA shows a significant effect of gap in the percentage of ASR inhibition $(F(8,171)=43.53, p<0.0001)$, together with a strong effect of group $(F(1,171)=20.3, p<0.0001)$, nevertheless, an interaction between gap and group was not present $(F(8,171)=1.14, p=0.34)$. 
A
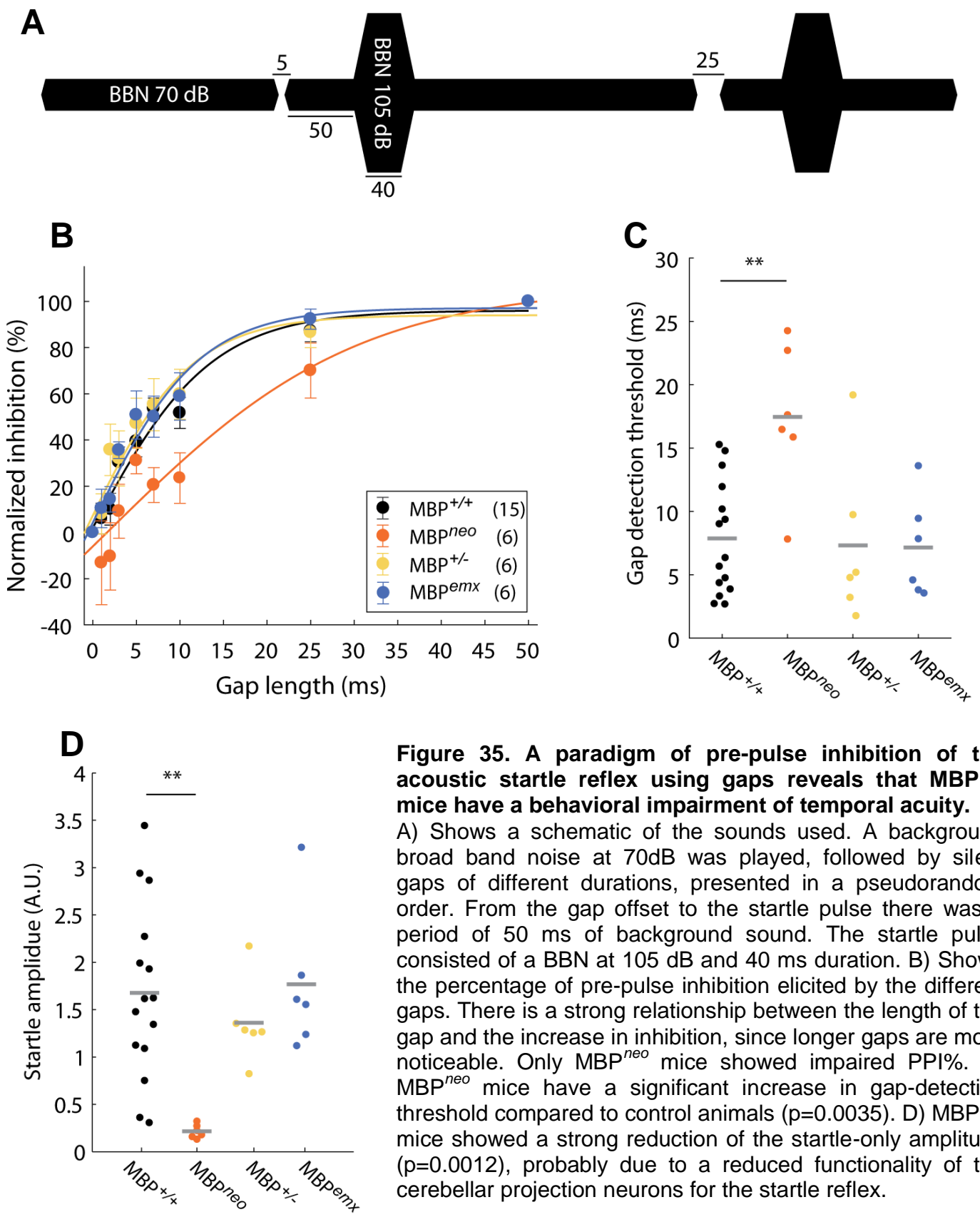

Figure 35. A paradigm of pre-pulse inhibition of the acoustic startle reflex using gaps reveals that $\mathrm{MBP}^{\text {neo }}$ mice have a behavioral impairment of temporal acuity.

A) Shows a schematic of the sounds used. A background broad band noise at $70 \mathrm{~dB}$ was played, followed by silent gaps of different durations, presented in a pseudorandom order. From the gap offset to the startle pulse there was a period of $50 \mathrm{~ms}$ of background sound. The startle pulse consisted of a BBN at $105 \mathrm{~dB}$ and $40 \mathrm{~ms}$ duration. B) Shows the percentage of pre-pulse inhibition elicited by the different gaps. There is a strong relationship between the length of the gap and the increase in inhibition, since longer gaps are more noticeable. Only $\mathrm{MBP}^{\text {neo }}$ mice showed impaired PPI\%. C) $\mathrm{MBP}^{\text {neo }}$ mice have a significant increase in gap-detection threshold compared to control animals $(p=0.0035)$. D) MBP ${ }^{\text {neo }}$ mice showed a strong reduction of the startle-only amplitude $(p=0.0012)$, probably due to a reduced functionality of the cerebellar projection neurons for the startle reflex.

Interestingly, heterozygote animals mice from the $\mathrm{MBP}^{\text {shi }}$ line, which have a loss of $50 \% \mathrm{MBP}\left(\mathrm{MBP}^{+/}\right)$, do not show a deficit in PPI of the ASR (ANOVA, F $(1,171)=2.21, \mathrm{p}<0.13$ ). It seems that a minimum amount of myelin loss is needed to impair gap detection at the behavioral level.

In addition, some studies have related intra-cortical processes that are necessary for gap detection (Weible et al., 2014a, 2014b). One question that arose was whether cortical- 
specific loss of myelin would have similar effects in the behavioral detection of gaps. I used the $\mathrm{MBP}^{e m x}$ mice to test this behavior. In this case, I also did not see any difference between control and $\mathrm{MBP}^{\mathrm{em} x}$ mice for the PPI (ANOVA, $\left.\mathrm{F}(1,171)=1.36, \mathrm{p}=0.24\right)$. I then obtained the specific values of gap detection for all the animals, and observed a very similar effect. Comparing control animals with $\mathrm{MBP}^{\text {neo }}$, we can observe a significant increase in the gapdetection threshold (Figure 35-C) $(p=0.0035)$, whereas the comparison of gap-detection thresholds between control animals and $\mathrm{MBP}^{+-}$or $\mathrm{MBP}^{e m x}$ mice yielded no significant difference ( $p=0.66, p=0.78$ respectively). Additionally, a significant difference in gap-detection threshold was seen between $\mathrm{MBP}^{\text {neo }}$ and $\mathrm{MBP}^{+-}(\mathrm{p}=0.017)$, and between $\mathrm{MBP}^{\text {neo }}$ and $\mathrm{MBP}^{e m x}$ $(p=0.0052)$. This means that a strong loss of myelin ( 80\%) elicits temporal acuity deficits in mice, consistent with the electrophysiological measurements of responses to gaps in the ACx. Interestingly, I observed also a strong reduction in the amplitude of the only-startle response of $\mathrm{MBP}^{\text {neo }}$ mice $(\mathrm{p}=0.0012)$ (Figure 35-D). No differences were seen between control animals and $\mathrm{MBP}^{+-}$or $\mathrm{MBP}^{e m x}$ mice $(\mathrm{p}=0.43, \mathrm{p}=0.83$ respectively). This means that the $\mathrm{MBP}^{\text {neo }}$ mice tend to startle less compared to control animals, when a loud sound is presented without a pre-pulse. This effect was striking, and I hypothesize is caused by an abnormality in the motor-related circuit that elicits the reflex.

\subsubsection{Gap-detection using the AudioBox in $\mathrm{MBP}^{\text {neo }}$ mice}

PPI of the ASR tests spontaneous responses related to a reflex. I was interested in testing gap-detection using a paradigm that resembled a more naturalistic environment. For this reason, I used an automated system for mice behavior: the AudioBox (NewBehavior, TSE systems) (de Hoz and Nelken, 2014) (see Materials and Methods section II.IX.II). Briefly, in the AudioBox, mice leave in a large home-cage in a large social group. Animals have food ad libitum, but for drinking water, they need to enter the sound box, which has a corner where they are recognized by the system because each animal was tagged previously with a transponder ID on the neck. Above the corner, there is a speaker, with which animals can be exposed to specific sounds and need to learn to discriminate them and behave accordingly. 'Safe' sounds carry the meaning of the possibility of drinking water, so animals can safely nosepoke and get water. 'Conditioned' sounds have a negative meaning; when animals hear them, they should not try to get water, otherwise they will get an air puff. In this case, I used a BBN continuous sound as the 'safe' sound and a sound with a gap recognizable gap of 50 ms was used as the 'conditioned' sound. Mice were then tested for the detection of different 
gap-lengths in the sound (each of them also conditioned to avoid generalization of avoiding nose poking to any new sound).

Because the $\mathrm{MBP}^{\text {shi }}$ mice have a very strong motor impairment, and usually tend to be more stressed than control mice, I was not able to use this model in the AudioBox. Nevertheless the partial dysmyelination mice did not show any motor impairment (data not shown) so they were suitable for this paradigm. First, the basic behavior in the AudioBox was tested between the two groups. I did not find a difference between the mean visit number per day between control and $\mathrm{MBP}^{\text {neo }}$ animals $(\mathrm{p}=0.32)$ when using the average of all days for the duration of the experiment (see Figure $36-\mathrm{A}$, control in black vs $\mathrm{MBP}^{\text {neo }}$ in orange, total duration depicted by ' $t$ '). Nevertheless, there was a significant difference on the average visit number per day, when considering only the first 4 days of exposure to the AudioBox $\left(\mathrm{p}=0.012\right.$ ) (see Figure $36-\mathrm{A}$, control in gray vs $\mathrm{MBP}^{\text {neo }}$ in peach, first 4 days depicted by ' 4 '). I did not observe a difference in the mean visit number per day in control animals when comparing the total duration of the experiment ( $\sim 0$ days) and only the first 4 days $(p=0.17)$. Nevertheless, $\mathrm{MBP}^{\text {neo }}$ mice showed a reduction in the mean number of visits per day in the first four days compared to the total duration $(p=0.015)$ (mutant total duration in orange vs mutant first 4 days in peach). This means that when the animals were first exposed to the AudioBox, during the initial habituation period of 4 days, $\mathrm{MBP}^{\text {neo }}$ mice did significantly less visits to the corner than control littermates. This could represent a behavioral problem with novelty coping in $\mathrm{MBP}^{\text {neo }}$ mice. Nevertheless, they only showed this behavior for a brief period at the very beginning of the experiment, and afterwards, they did similar amount of visits per day. This was especially important because mice that make fewer visits would be less exposed to the sounds, and this could have an effect in their performance. To make sure the comparisons I am doing between groups are accurate, I measured the average number of visits that the animals made per day for the gaps used. I found no differences between control and $\mathrm{MBP}^{\text {neo }}$ animals in the average amount of visits per day, in any of the gaps tested, which means both groups had an equal exposure to the gaps (ANOVA, $F(1,162)=1.11, p=0.29$ ) (Figure 36-B). There was also no effect of the gap to which the animals were exposed, in the amount of visits they were doing (ANOVA, $F(8,162)=0.56, p=0.81$ ), which means that even though the animals sometimes had a challenging task when detecting small gaps, this did not affect their motivation to continue doing visits to the corner. 
A

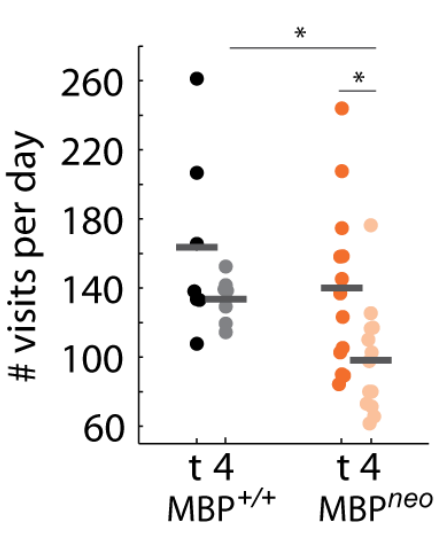

C
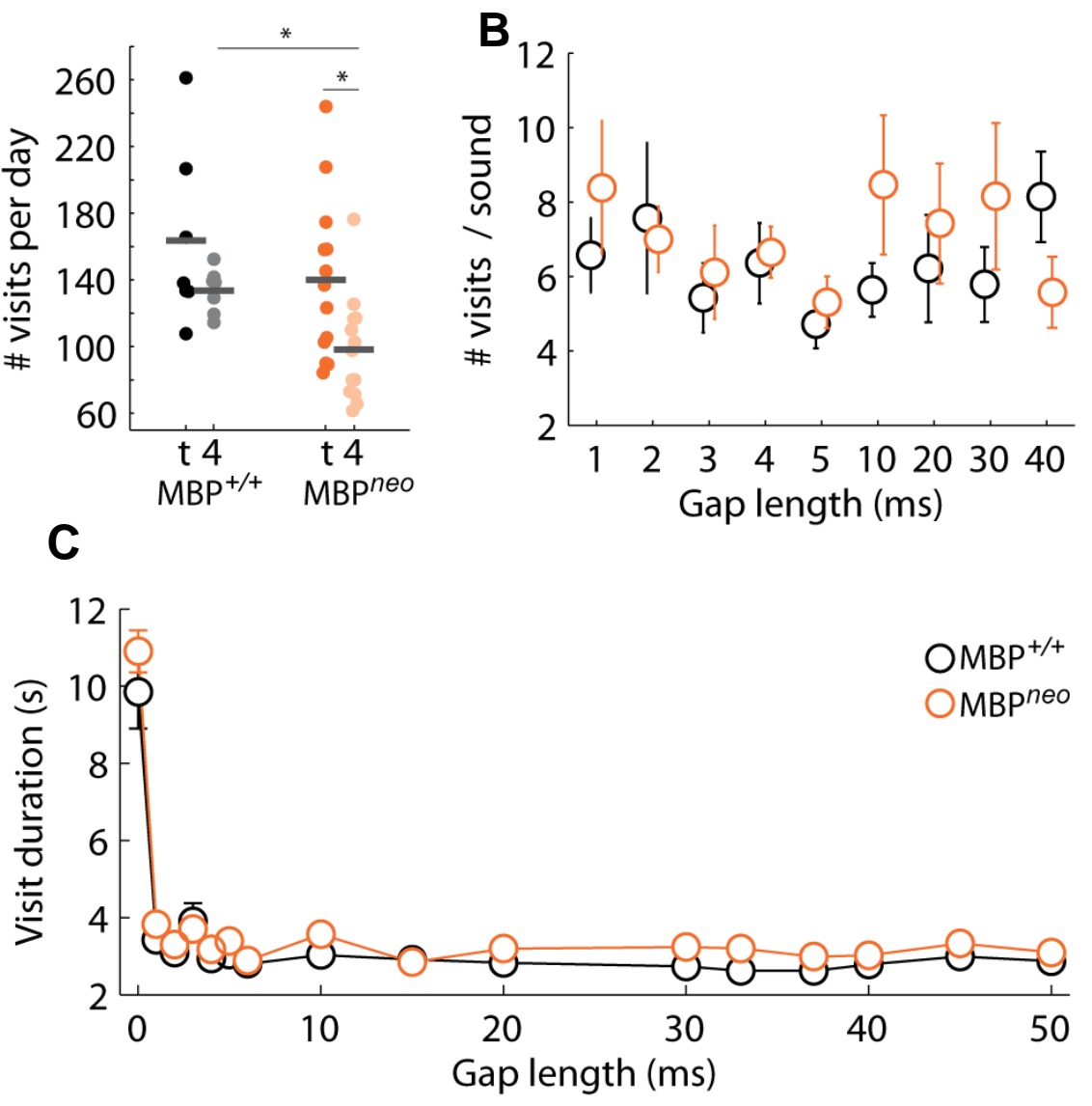

D

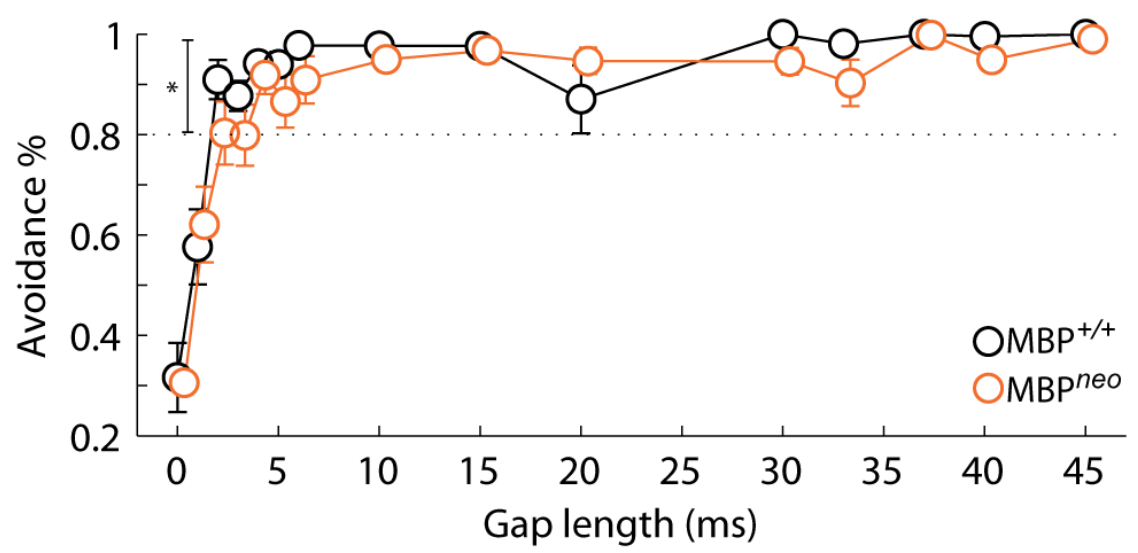

Figure 36. Behavioral gap-detection in the AudioBox is impaired in $\mathrm{MBP}^{\text {neo }}$ mice.

A) Quantification of the total average visits per day for the entire duration of the experiment ( $x$ axis: ' $t$ ') for control (black) and MBP ${ }^{\text {neo }}$ mice (orange). The average number of visits per day for the first 4 days of habituation (x axis: '4') is represented for control (gray) and mutant (peach) animals. No differences between groups were seen for the total experimental duration, but a significant difference was seen between groups in the first 4 days of habituation $(p=0.012)$. Gray bars show the mean of each condition. B) Shows the quantification of the average number of visits per sound. No differences were seen in any of the gaps tested for both groups (ANOVA, $p=0.29$ ). In $\mathrm{C}$ ) the quantification of visit duration represents a measure of perceptual difficulty for control (black) and mutant (orange) animals. A significant increase in the visit duration was seen overall the gaps tested (ANOVA, $\mathrm{p}=0.0017$ ). D) The quantification of avoidance behavior revealed that above the gap-detection threshold ( $>1 \mathrm{~ms}$ gap), there is a significant difference between control (black) and mutant (orange) animals (ANOVA, $p=0.014$ ) showing the MBP ${ }^{\text {neo }}$ mice reduced avoidance, meaning that they have difficulties detecting gaps in sound and therefore have temporal acuity deficits in this behavioral paradigm. 
A way of quantifying the perceptual difficulties of the task the animals are performing is by measuring the duration of the visits to the corner, depending on the sound the animals were exposed to.

I measured the mean time that the animals spent on each visit depending on the sound that was being played (Figure 36-C) and found that overall, $\mathrm{MBP}^{\text {neo }}$ mice tend to spend more time in the corner (ANOVA, $F(1,288)=10.08, p=0.0017$ ). In addition, I found a strong dependence of the gap length on the visit length (ANOVA, $F(15,288)=80.16, p<0.0001$ ), meaning that animals recognized the gaps that were conditioned (all except $0 \mathrm{~ms}$ ), and were leaving the corner faster when they heard those sounds (in $\sim 3$ seconds) compared to when they heard the 'safe' sound ( 10 seconds).

Finally, I measured the behavioral output of the gap-detection task by quantifying the number of visits where animals did not do a nosepoke (avoidance percentage) depending on the sound they were hearing. The avoidance percentage increases proportionally with the increase in gap-length (ANOVA, $F(14,270)=35.21, p<0.0001$ ) (Figure 36-D), meaning that animals learnt to recognize that sounds with a gap meant not to drink water, and they avoided trying to get water effectively. In addition, we see a strong categorization of the gap lengths, meaning that, in fact, smaller gaps were more difficult to recognize in this behavioral setup, than longer gaps. We can also observe that from gaps of 2 to $6 \mathrm{~ms}$, there is a tendency of the mutant animals to have lower avoidance percentages, being closer to the threshold line (dotted horizontal line) that represents $80 \%$ of avoidance, compared to control littermates. Nevertheless, at $1 \mathrm{~ms}$ gap, it seems that there is no difference between groups, that could be related to the fact that the threshold for gap detection is higher than $1 \mathrm{~ms}$ in mice. When comparing both groups above the gap-detection threshold (2 ms), which has also been reported for mice in other studies (Radziwon et al., 2009; Strenzke et al., 2016; Walton et al., 1997), taking gap-lengths from 2 ms until 45 ms, I found a significant difference in the percentage of nose-poke avoidance, having the $\mathrm{MBP}^{\text {neo }}$ mice significantly lower avoidance percentage (ANOVA, $\mathrm{F}(1,234)=6.07, \mathrm{p}=0.014$ ), meaning that $\mathrm{MBP}^{\text {neo }}$ mice also show a temporal acuity impairment in this behavioral paradigm. In this case, I was able to find a correlate of the electrophysiological temporal acuity abnormalities seen in these mutants, with the gap-detection measurement from the inhibition of the startle reflex, and with a naturalistic behavioral setup. With this, I confirm with full certainty that $\mathrm{MBP}^{\text {neo }}$ mice show reduced temporal acuity, which has a strong neuronal correlate in the auditory cortex. 


\subsection{Discussion}

This work focuses on assessing the role that oligodendrocytes play in circuit function in the brain. Specifically, in this chapter, I aimed to address this question by studying how the processing of different sound properties changes in the auditory system upon dysmyelination. I covered aspects of basic sound processing in subcortical auditory stations and focused on doing an in-depth assessment of cortical higher-order areas. In this chapter, I presented the results of three mouse models: with a) complete $\left(\mathrm{MBP}^{\mathrm{sh}}\right)$, b) partial $\left(\mathrm{MBP}^{\text {neo }}\right)$, and $\mathrm{c}$ ) areaspecific $\left(\mathrm{MBP}^{\mathrm{em} x}\right)$ loss of myelin. Overall, the main observation was that dysmyelination causes striking deficits in temporal processing in the cortex, especially in features that are associated with speech discrimination in humans. To test the perceptual impact of poor neural temporal processing, I did behavioral tests that paralleled the defects observed. These experiments revealed a correlation between neural temporal acuity problems and changes in perception due to partial dysmyelination. Also, ex vivo white matter recordings were performed in order to understand the differences between gray and white matter processing and with the goal of finding common molecular mechanisms of the abnormalities observed.

\subsubsection{Functional consequences of the loss of MBP in grey and white matter}

\subsubsection{Basic auditory processing is affected with different levels of dysmyelination}

The auditory system is a preferred subject of studies exploring the role of myelin. This sensory system is known for its high temporal precision, something that one would expect to be among the first properties to be lost in a de/dysmyelinated circuit. To date, several studies have reported general auditory abnormalities in different myelin deficient models, typically using quantifications from auditory brainstem responses. These studies found an overall increase in auditory evoked response latencies in different CNS myelin mutant rodents (Carpinelli et al., 2014; Ito et al., 2004; Kanzaki et al., 1985; Kim et al., 2013a; Naito et al., 1999; Roncagliolo et al., 2000; Shah and Salamy, 1980). Different myelin patterns help the

synchronous firing of fibers that have different lengths (Lang and Rosenbluth, 2003; Salami et al., 2003; Seidl, 2014). Several studies have indeed described precise patterns of myelination in auditory pathways related to sound localization, which is achieved by minimal differences in the relative timing of spike arrival (Ford et al., 2015; Seidl, 2014; Seidl et al., 2014). 


\section{Peripheral processing is not affected}

One important issue to address was to understand if the mutations generated an impairment in peripheral auditory processing that could cause generalized auditory abnormalities. For this, I analyzed two aspects: the ABR wave I characteristics and the hearing thresholds. Wave I of the ABRs gives a reliable measure of the integrity status of the auditory nerve fibers (ANF) (Sergeyenko et al., 2013). A decrease in wave I amplitude is proportional to the level of ribbon synapse loss in the cochlea (Jean et al., 2018; Kujawa and Liberman, 2009) and associated with the loss of ANF afferent terminals (Lopez-Poveda and Barrios, 2013). In none of the models presented here, I observed a significant decrease of wave I amplitude (Figure 17-D, Figure 30-D, Figure 27-D), suggesting that peripheral processing is not affected by dysmyelination. Additionally, none of the mutants tested had a significant difference in hearing thresholds (Figure 17-B, Figure 30-B, Figure 27-B), which indirectly shows that outer hair cell function was normal in all cases. Interestingly, in the MBP ${ }^{\text {shi }}$ mice, I observed a delay in ABR wave I, which depicts the response of the auditory nerve. I attribute this to the fact that the latter part of the auditory nerve is myelinated by oligodendrocytes and not Schwann cells (Wan and Corfas, 2017; Xing et al., 2012) and the MBP defect in oligodendrocytes might be causing this latency increase. Overall, the data indicates that there is no AFN loss caused by dysmyelination and that the deficits observed centrally are not inherited from peripheral problems.

\section{Speed matters}

Undoubtedly, one key role of myelin is to regulate conduction velocity. It is therefore not surprising that I observed strong latency increases in the models with dysmyelination along the auditory system. Both subcortical and cortical areas in the $\mathrm{MBP}^{\text {shi }}$ mice showed a latency increase that grew as the signal advanced through the different auditory stations (see Figure 17-C and Figure 18). $\mathrm{MBP}^{\text {neo }}$ mice showed a similar phenotype, with less involvement from the periphery (Figure 30-C). For both cases, this suggests there is a cumulative delay, due to dysmyelination at every neuron through the auditory system, being latter stations more affected than earlier ones, because of the distance the signal has to travel. The conduction velocity in the optic nerve, a white matter tract, is reduced by $\sim 42 \%$ in $\mathrm{MBP}^{\text {shi }}$ mice and by $\sim 28 \%$ in $\mathrm{MPB}^{\text {neo }}$. Interestingly, the dependency of CV with myelin loss does not appear to be linear (Figure 32-C). Since $\mathrm{MBP}^{\text {shi }}$ mice develop secondary defects to the loss of myelin, these could account for a further decrease in CV. The most plausible explanation here is that 
the increased amount of small caliber axons seen in MBP ${ }^{\text {shi }}$ optic nerves (Kirkpatrick et al., 2001 ) is slowing further down the conduction of action potentials.

\section{It is not only about speed}

Both $\mathrm{MBP}^{\text {shi }}$ and $\mathrm{MBP}^{\text {neo }}$ mice showed impaired processing in early brainstem regions, observed by an abnormal shape of ABR wave II and/or III (early brainstem), which appeared merged and with reduced amplitude (see Figure 17-A, Figure 30-A). These results correlate with observations made in MS or PMD patients, where a lack of wave III has been reported (Chiappa et al., 1980; Henneke et al., 2010). These effects might result from the reduced synchrony of signals converging to these areas (Moser and Starr, 2016). Additionally, both $\mathrm{MBP}^{\text {shi }}$ and $\mathrm{MBP}^{\text {neo }}$ mice showed an increased gain in later brainstem regions (represented in waves IV and V) (Figure 17-F and G and Figure 30-G). This gain increase has been reported in mice deprived of auditory stimuli (Clarkson et al., 2016), with aging (Sergeyenko et al., 2013) and in MS patients (Chiappa et al., 1980), which are directly or indirectly related to changes in myelin (Lasiene et al., 2009; Peters, 2009; Sinclair et al., 2017; Xing et al., 2012). Central auditory gain increase is predicted to be partly caused by loss of ANF observed as a strong reduction of wave I amplitude (Schaette and McAlpine, 2011). In my study, since I did not observe changes in wave I amplitudes, I relate the gain increase in later brainstem regions to the decrease in amplitude of the early auditory brainstem regions.

The results I obtained suggest first of all, that a loss in conduction velocity not only slows down signal propagation but affects the synchrony of converging signals, which might have a substantial effect in the coding of information. This might be especially relevant in systems that require high temporal precision, or for multisensory processing since different paths might have special adaptations to regulate coincidence detection. Furthermore, auditory stations can undergo compensatory mechanisms to ensure adequate processing. The increase in gain, indicates neuronal hyperexcitability, and it is occurring due to 'homeostatic plasticity' (i.e., a series of processes that refine neural circuits in an experiencedependent manner) (Turrigiano, 1999). Given this, it is possible that dysmyelination is causing asynchronous abnormal responses in the early brainstem regions which are leading to homeostatic plastic changes in latter brainstem regions to ensure signal fidelity at higherorder stations. 


\section{What happens in the cortex?}

To assess the effect of dysmyelination in higher-order auditory processing, I recorded extracellular responses from the auditory cortex. I did not observe any differences in amplitude neither in the $\mathrm{MBP}^{\text {shi }}$ nor the $\mathrm{MBP}^{\text {neo }}$ mice (see Figure 18 and Figure 31). Responses from the inferior colliculus (one station before the cortex) had a trend towards higher amplitudes, an observation that correlates with the gain increase seen in this area and previously discussed.

Interestingly, unlike the $\mathrm{MBP}^{\text {shi }}$ or $\mathrm{MBP}^{\text {neo }}$ mutants, $\mathrm{MBP}^{\text {emx }}$ mice showed overall strong reduction in the response amplitudes. In these mice dysmyelination is probably restricted to the latter portion of the thalamo-cortical projections, a relatively small section. The possibility of generating compensatory mechanisms within this small region is probably very limited. In addition, the reduction of response amplitude could be reflecting conduction blocks, which happen because the axons have an abrupt change in the speed of action potential propagation and ion conductance. Nevertheless, this is all speculation based on the assumption that dysmyelination in the $\mathrm{MBP}^{e m x}$ is restricted to the latter portion of thalamocortical projections.

Overall, my results suggest that the detrimental effects of desynchronized responses due to dysmyelination in early auditory stations are compensated at the level of the late brainstem, and the cortical output is not different in amplitude compared to control animals in the $\mathrm{MBP}^{\text {shi }}$ and $\mathrm{MPB}^{\text {neo }}$ mice. However, when dysmyelination is limited to a specific area (as in $\mathrm{MBP}^{\mathrm{emx}}$ ), these compensatory mechanisms are not activated and a strong dampening in spiking activity is observed, probably due to conduction blocks. Interestingly, the cortical responses do not echo subcortical hyperexcitability, suggesting that additional filtering processes must be happening between the inferior colliculus and the cortex (or within the cortex) since the cortical responses do not echo subcortical hyperexcitability.

\section{Is the cortex in a hyperexcitable state?}

The gain increase to compensate for the loss of synchrony leads to hyperexcitability in subcortical structures. However, it could also be due to changes in neuronal properties of neurons because of myelin loss. For instance, it has been reported that dysmyelinated axons have an increase in the expression of sodium channels along naked axons (Noebels et al., 1991; Westenbroek et al., 1992), the number of clusters is increased (Sinha et al., 2006) or 
there is a change in the isoforms expressed (Boiko et al., 2001). These differences can render de/dysmyelinated axons different excitability properties compared to normally myelinated axons, a mechanism that might potentiate their coding abilities.

I observed a strong reduction of the length of AnkG in the AIS of MBP ${ }^{\text {shi }}$ mice (Figure 20). It has been reported that a mechanism to cope with demyelination-induced hyperexcitability of cortical neurons is trough plastic changes in the axon initial segment (Hamada and Kole, 2015; Susuki and Kuba, 2016). In general, the AIS can be shifted proximally or distally from the soma, or can change in length to modulate axonal excitability (reviewed in, (Jamann et al., 2018; Rasband, 2011; Yamada and Kuba, 2016). Reductions in AIS length have been associated with hyperexcitable states, where the neurons try to compensate with a reduction on the area that can elicit an action potential to reduce their internal excitability (Evans et al., 2015; Grubb and Burrone, 2010; Susuki and Kuba, 2016). This reduction in AIS suggests that the $\mathrm{MBP}^{\text {shi }}$ cortical axons reside in a hyperexcitable environment.

I also tested whether potassium channels in the AIS were similarly affected, since these are also implicated in excitability. Kv7 channels line the AIS and play an important suppressive role in regulating excitability, resting membrane potential and action potential threshold (Shah et al., 2008). The length of the $\mathrm{K}_{\mathrm{v}} 7.3$ channels was similar between MBP ${ }^{\text {shi }}$ and control animals (Figure 20). The fact that I saw a normal distribution of $\mathrm{K}_{\mathrm{v}} 7.3$ channels, suggests that the plastic changes observed in the AIS may affect the distribution of $\mathrm{Na}_{v}$ channels instead, since limiting the presence of $\mathrm{Na}_{v}$ channels at the AP initiation site to reduces excitability (Evans et al., 2015). A more detailed analysis of the distribution of these channels still needs to be assessed to confirm this assumption. In addition, the direct measurement of $\mathrm{K}_{\mathrm{v}} 7$ channels expression must be performed to confirm no abnormalities.

The data suggests that dysmyelinated neurons in the cortex are regulating their excitability through a shortening in the AIS. Nevertheless, this shortening can also be associated with a developmental stagnation due to the lack of compact myelin, since shorter AIS have also been observed in pre-myelinating stages (Gutzmann et al., 2014). In demyelinating conditions, the AIS does not change in length in the cortex (Clark et al., 2016; Hamada and Kole, 2015), but it shifts towards the soma and increases the length of the $K_{v} 7.3$ channels (Hamada and Kole, 2015). Additionally, animals with experimental autoimmune 
encephalomyelitis (EAE) show a significant reduction of AIS length and number (Clark et al., 2016). These are all mechanisms used to compensate for the hyperexcitable state the neurons reside on, due to the loss of myelin. These results suggest that AIS compensatory mechanisms upon demyelination and dysmyelination are different and further characterization of the specific AIS changes in the $\mathrm{MBP}^{\text {shi }}$ is necessary to understand this process. For example, the measure of not only the length of the AIS, but the distance from the soma might be a useful quantification to understand the plastic processes the AIS undergo upon dysmyelination.

Taken together, the results suggest that early auditory brainstem regions are affected due to the desynchronization of inputs from dysmyelinated fibers. Nevertheless, later auditory stations are able to compensate for the desynchronization by amplifying the outputs they send to further auditory stations. In addition, cortical neurons might be regulating hyperexcitable states through the shortening of the AIS. These results highlight the amazing complexity of the auditory system and the internal cooperative interactions that take place to ensure proper information processing.

\subsubsection{The effects of different levels of dysmyelination in white matter}

The degree of white matter dysfunction was assessed by doing ex vivo optic nerve recordings and measuring the characteristic of the recorded compound action potentials. CAP shapes of mutant $\mathrm{MBP}^{\text {shi }}$ and $\mathrm{MBP}^{\text {neo }}$ animals were severely impaired, compared to control animals. I observed a reduction in response amplitude and area of the CAP, proportional to the amount of MBP loss (Figure 21 and Figure 32). The observations made here using the CAP of the optic nerve are very similar to what has been reported in the spinal cord (Sinha et al., 2006). A reduction in CAP amplitude means that lower number of axons is responding to the stimulation. Interestingly, the $\mathrm{MPB}^{\text {neo }}$ mice showed an intermediate phenotype between control and $\mathrm{MBP}^{\text {shi }}$ mice. The reason why less number of axons is responding is not yet clear. One must keep in mind that the CAP measurement is fundamentally different from that obtained from the cortex. ON activity is elicited through electrical stimulation of the totality of the nerve. Since axons are largely naked in the $\mathrm{MBP}^{\text {shi }}$ mice, it is possible that interactions between axons affect excitability. In the cortex, activity is evoked by sound presentation and thus the amount of axonal fibers activated is reduced with respect to the ON setting, and this might have the paradoxical effect of allowing stronger responses at the level of the cortex. One very interesting observation that gave me insight into the reason of the amplitude 
reduction in the MBPshi CAPs was the strong hyperpolarization that arises from the reduction of MBP. The $\mathrm{MBP}^{\text {neo }}$ mice haven an intermediate phenotype compared to control and $\mathrm{MBP}^{\text {shi }}$ in this hyperpolarizing phase, which is nonexistent in the control animals. The strong hyperpolarization is related to changes in the potassium currents caused, in MBP ${ }^{\text {shi }}$ mice, by the elongation of the nodes (Rasband et al., 1999a; Sinha et al., 2006) and overexpression of potassium channels like $\mathrm{K}_{\mathrm{v}} 1.1$ and $\mathrm{K}_{\mathrm{v}} 1.2$ subunits (Sinha et al., 2006; Wang et al., 1995a). The CAP amplitude decrease and the hyperpolarizing phase in $\mathrm{MBP}^{\text {shi }}$ axons was partially compensated upon the addition of 4-AP (Figure 26), a selective $\mathrm{K}_{\mathrm{v}} 1.1$ and $\mathrm{K}_{\mathrm{v}} 1.2$ blocker, suggests that the hyperpolarizing phase is related to an over-activation of $K_{v} 1$ channels. $K_{v} 1$ channels are usually located at the juxtaparanodes (Stathopoulos et al., 2015), and upon dysmyelination, they become more accessible. Because of both the overexpression of $K_{v} 1$ channels and the increase in their availability, $\mathrm{K}_{v} 1$ channels might be preventing sodium currents and thus both shunting the CAP amplitude and causing an increase in hyperpolarization. If this hypothesis is true, these results suggest that in $\mathrm{MBP}^{\text {neo }}$ mice, a mislocalization and exposure of Kv1 channels is happening, nevertheless, in a lesser extent compared to the $\mathrm{MBP}^{\text {shi }}$.

Changes in the response of axons can have marked effects for information coding. ON responses from MBPshi showed fatigability when stimulated at high rates (Figure 25). Similar to other studies (Sinha et al., 2006), white matter tracts in the MBP ${ }^{\text {shi }}$ mice seem less suited to sustain firing at high rates. Since I hypothesized that the fatigability was probably due to the hyper-activation of the $\mathrm{K}_{\mathrm{v}} 1$ channels, I used a high-rate stimulation paradigm, in combination with the influx of 4-AP to reduce the hyperpolarization and asses if the fatigability phenotype could be reversed. Nevertheless, very preliminary results showed that the drug did not rescue the fatigability phenotype (Figure 26). In the case of the $\mathrm{ON}$, I hypothesize that fatigability could be caused by the lower amount of $\mathrm{Na}_{v} 1.6$ channels in $\mathrm{MBP}^{\text {shi }}$ nerves(Boiko et al., 2001), which are important for maintaining high firing rates (Osorio et al., 2010; Van Wart and Matthews, 2006a).

Overall, different effects of the loss of myelin during development can be observed by studying white matter tracts. Some of them arise from the direct loss of myelin, while others are related to axonal architectural modifications regulated by axo-glial interactions. In general, these effects appear different from what was observed in gray matter. Dysmyelinated white matter axons tend to have reduced response amplitudes, possibly due to shunting of sodium 
currents upon activation of repolarizing potassium channels that are more accessible in a naked axon. These channels could be also responsible for the appearance of a strong hyperpolarizing phase in the CAP of the optic nerve. Reduced conduction velocity and impaired excitability might have to do with two factors, obviously the lack of myelin on one hand, and also the changes in axon caliber.

\subsubsection{Total myelin loss impairs temporal reliability and acuity in the ACx}

It is well established that myelin is essential for the accurate encoding of signal timing and that the loss of compact myelin may reduce temporal precision and reliability of firing (Kim et al., 2013a). It is expected then, that myelin deficiencies cause auditory dysfunctions, especially in the temporal spectrum of sound processing. At the population level, fibers that require a highly precise timed response may not be able to code the correct temporal envelope if there are conduction delays or blocks. For this reason, I focused on two types of temporal processing: temporal acuity, assessed by the detection of gaps in sound, and temporal reliability, using the measurement of spike synchrony during rate coding.

\subsubsection{Dysmyelination reduces temporal reliability, measured by rate coding in the ACx}

Neurons in the auditory cortex have context-dependent flexibility. For example, the previous stimulus history can influence spiking activity (Ulanovsky et al., 2004), a process known as neuronal adaptation. As beautifully defined by Cardanini and Ferster: 'adaptation acts as a gain control mechanism that maximizes the sensitivity of cortical cells to the average [ ] of their most recent stimuli' (Carandini and Ferster, 1997). In this study I tested the sensitivity of cortical neurons to the stimulus history by using two paradigms, the click tracing protocol, which gives a measure of temporal reliability, and by measuring stimulus specific adaptation to a tone with an oddball paradigm.

\section{Adaptation and temporal reliability}

Adaptation to sensory inputs influences coding properties (Gollisch and Herz, 2004), and represents an important process for higher-order processing, like auditory scene analysis (the property to perceive complex auditory environments) (Bregman, 1994), or the formation of auditory memory (Näätänen et al., 2001). Since the goal of this study is to disentangle the 
roles of myelin in higher-order sensory processing, I studied rate coding in the cortex by measuring the response adaptation to a train of 10 clicks, presented at different rates ( 5 to 20 $\mathrm{Hz}$ ) in the $\mathrm{ACx}$ of $\mathrm{MBP}^{s h i}, \mathrm{MBP}^{\text {neo }}$ and $\mathrm{MBP}^{\mathrm{emx}}$ mice. The greatest reduction on temporal reliability was observed in $\mathrm{MBP}^{\text {shi }}$. Often, the responses to clicks in $\mathrm{MBP}^{\text {shi }}$ mice showed a strong dampening at around click number 6 , which resulted in a total ablation of response to further clicks. In these mutants, differences in spike synchrony between groups were observed until the cutoff of rate coding in the ACx (Figure 23). There is a gradual effect of dysmyelination in rate coding in the $\mathrm{ACx}$, since the $\mathrm{MBP}^{\text {neo }}$ had significant defects until $8 \mathrm{~Hz}$. (Figure 34), while $\mathrm{MBP}^{\text {shi }}$ presented them until $14 \mathrm{~Hz}$. Mice with only-cortical dysmyelination $\left(\mathrm{MBP}^{\mathrm{em} x}\right.$ ) were also impaired in rate coding, and had a very similar phenotype to the MBP ${ }^{\text {shi }}$, showing defects until $10 \mathrm{~Hz}$ stimulation rate (Figure 29). Interestingly, rate coding difficulties have also been reported in MS patients (Chiappa et al., 1980). Additionally, the Long-Evans Shaker (LES) rats, a dysmyelination model, have decreased firing reliability at high frequencies (Kim et al., 2013a, 2013b) in the brainstem, suggesting that adequate myelin structure is necessary for temporal reliability. Since the LES rats and the MBP ${ }^{\text {shi }}$ mice are similar models, the observation of impaired cortical rate coding in the $\mathrm{MBP}^{\text {shi }}$ could be related to a reliability deficit in the brainstem. Preliminary extracellular recordings from the inferior colliculus showed normal rate coding in $\mathrm{MBP}^{\text {shi }}$ mice. Nevertheless, the use of intracellular or cell-attached techniques could provide more information regarding rate coding in stations where the cutoff rate is high.

\section{What could be regulating deficient rate coding in dysmyelination?}

The intrinsic electrical properties of the neurons might be responsible for the coding of temporal information. It has been suggested that cells with particular voltage-sensitive potassium channels fire to high rates while cells that lack those channels could only follow low frequencies (Burger and Pollak, 1998). It is known that the ratio of $\mathrm{Na}_{v} 1.2$ and $\mathrm{Na}_{v} 1.6$ changes during development. In early stages, $\mathrm{Na}_{v} 1.2$ channels dominate unmyelinated axons and as myelination progresses, $\mathrm{Na}_{v} 1.6$ gradually replaces $\mathrm{Na}_{v} 1.2$ channels (Boiko et al., 2001; Van Wart and Matthews, 2006b). Nevertheless, in MBP shi mice, since myelination signals are impaired, this ratio is not reached in adulthood, and a strong reduction of $\mathrm{Na}_{\mathrm{v}} 1.6$ channels is observed in these mutants, while $\mathrm{Na}_{v} 1.2$ channels are extensively expressed (Boiko et al., 2001). Since $\mathrm{Na}_{v} 1.6$ channels are implicated in repetitive firing or certain neuron types (Van Wart and Matthews, 2006a), and $\mathrm{Na}_{v} 1.2$ show stronger inactivation at higher stimulation (Rush et al., 2005), it is likely that axons from MBP ${ }^{\text {shi }}$ mice, with a higher 
concentration of $\mathrm{Na}_{v} 1.2$ channels are not able to fire to high stimulation rates, probably because they have longer absolute refractory periods. Furthermore, the overexpression and ectopic expression of potassium channels would account for larger relative refractory periods. Refractoriness is a general property of neurons. It refers to a decrease in spiking probability once an action potential has been fired (Avissar et al., 2013). This help the prevention of action potential backpropagation, and it usually lasts 1-2 ms (Rudnicki et al., 2015).

In addition to specific channels conferring biophysical properties for rate coding, two main mechanisms of adaptation have been proposed: one that has to do with changes at the output of the neuron (changes in voltage-dependence conductances) or tonic hyperpolarization (Ulanovsky et al., 2004). Adaptation generates a strong tonic hyperpolarization which affects synaptic excitatory inputs (Carandini and Ferster, 1997). I observed that $\mathrm{MBP}^{\text {shi }}$ mice in the optic nerve have a strong hyperpolarizing phase. If we assume that this large hyperpolarization is also present in cortical neurons, the fast repolarization of the axon could be prolonged, impeding the neurons to fire to another stimulus close in time and explains why they cannot follow continuous stimulation. Interestingly, a similar observation was made in a parallel model of dysmyelination in rats, which have a stronger fast-after hyperpolarization peak compared to controls in a brainstem synapse (Kim et al., 2013a).

\section{Stimulus-specific adaptation, a complex process that requires accurate rate coding}

To study how deficits in rate-coding affect more complex cortical processes I assessed stimulus-specific adaptation in the $\mathrm{MBP}^{\text {shi }}$ mice. SSA measurements allows to obtain information regarding the capabilities of coding in a system, since it requires the fast comparison of sensory information (Ayala and Malmierca, 2012; Ulanovsky et al., 2004). In this case, I observed higher levels of SSA in the cortex of MBP ${ }^{\text {shi }}$ mice compared to control animals (Figure 24). This effect is expected considering that $\mathrm{MBP}^{\text {shi }}$ mice have stronger rate adaptation in the cortex (Figure 23). Additionally, the results obtained confirm that frequency discrimination is not affected in $\mathrm{MBP}^{\text {shi }}$ mice, since SSA was present. I hypothesize that the increase in SSA is due to the reduced rate coding abilities of cortical MBP ${ }^{\text {shi }}$ neurons.

A comparable measurement of SSA in humans is mismatch negativity (MMN), which is an electric potential measured in the scalp, mainly generated in the ACx, and elicited by oddball paradigms. It is believed that, to a certain extent, SSA is the neural basis of MMN 
(Malmierca et al., 2015). In humans, MMN is pre-attentive and it is widely used for sounddiscrimination accuracy tests (Näätänen et al., 2001). Interestingly, MMN has been used as a cognitive test for MS patients (Santos et al., 2006), where it shows to be impaired (Jung et al., 2006) suggesting a role of myelin in deviance detection accuracy in humans. Furthermore, MMN is increased in children with propensity to develop schizophrenia (Bruggemann et al., 2013) and in people with post-traumatic stress disorder (Ge et al., 2011; Morgan and Grillon, 1999), two diseases that have been linked to myelin deficits (Chao et al., 2015; Chiappelli et al., 2015; Li et al., 2016; Xu and Li, 2011).

My data confirms that the reduction in firing reliability in the cortex due to myelin deficits might have a stronger impact when it comes to overall cortical processing. Since SSA does not depend only in the biophysical properties of specific neurons, but it requires the orchestration of network activity (Malmierca et al., 2015), these results suggest that dysmyelination might impair higher-order auditory processing.

\subsubsection{Dysmyelination reduces temporal acuity, measured by gap-detection in the ACx}

In addition to rate-coding, the capacity of detecting rapid variations in sounds, temporal acuity also happens in the cortex (Anderson and Linden, 2016). The most commonly used paradigm for testing temporal acuity is the detection of gaps in a sound, which gives a measurement of the sensitivity and time constants of the auditory system (Shailer and Moore, 1983).

\section{Detecting silent gaps in sound}

To measure temporal acuity in the cortex, I used a gap-detection paradigm. As expected, I observed an increase in spiking activity in the post-gap response, with the increase in gap length in control animals. Nevertheless, especially in $\mathrm{MBP}^{\text {shi }}$ mice, this property was severely affected (Figure 22, Figure 28, Figure 33). Even though the $\mathrm{MBP}^{\text {neo }}$ did not show a significant decrease in the detection of gaps per se (measured with the baseline vs post-gap significance value), they still showed reduced response amplitude (Figure 33-B). This means that there was certain spiking activity after the smallest gaps but the spiking probability of mutant neurons was lower than that of controls. Additionally, $\mathrm{MBP}^{e m x}$ mice showed a milder effect by the quantification of significant responses at the post-gap sound compared to baseline activity. This suggests that the gap-detection deficits measured in the cortex are not an accumulation of temporal deficits from the other auditory stations. Since I recorded form a 
population of neurons, this translates to a lower amount of neurons being able to repolarize fast enough to respond to the next stimulus that comes close in time. In accordance to my results, impairments in gap-detection have been related to the appearance of developmental deficits, during aging, and not seen due to hearing loss (Anderson and Linden, 2016; Eggermont, 2015; Weible et al., 2014a). In addition, MS patients have altered gap-detection thresholds, mechanism that might be accounted for their speech discrimination deficits (Valadbeigi et al., 2014, 2017). Taking this together, myelin seems to be playing a role in temporal acuity in the auditory system, which is a necessary processes for the accurate detection of speech (Hoover et al., 2015; Phillips, 1999).

If we think about the hypothesis that $\mathrm{MPB}^{\text {shi }}$ mice have longer refractory periods, similar mechanisms to the ones discussed for rate coding, could be accounting for the impairment in gap-detection. Auditory stimuli can happen along the refractory period, in which neurons are prevented from spiking, which could potentially affect the coding resolution of auditory information. The basic assumption is then, that in MBPshi mice, refractoriness is impaired and prevents the axons from firing after the gap, unless this is large enough.

One factor that might be impacting the level of response is the anesthesia status. It has been shown that anesthesia can change gap-evoked auditory responses in a dose dependent manner (Feng et al., 2007). Nevertheless, anesthesia was always administered according to individual weight, and I did not observe any differences in the 'anesthesia index' (total amount of anesthesia administered by the overall recording time) between controls and $\mathrm{MBP}^{\text {shi }}$ mice (see Appendix, Figure 51), which rules out an influence in anesthesia in the observed results.

Overall, dysmyelination affects temporal acuity, measured by the gap-detection protocol at the electrophysiological level in the auditory cortex. MBP ${ }^{\text {shi }}$ were the mutants that presented the strongest temporal acuity phenotype. In addition, a reduction in spiking probability after the gap was seen in the $\mathrm{MBP}^{\text {neo }}$ model, suggesting that even a milder form of dysmyelination can impair temporal acuity in the cortex at the electrophysiology level. The next question is whether the effects seen at the level of neuronal populations in the cortex have a correlate in the behavior of the animals. 


\subsubsection{Partial myelin loss is enough to impair temporal acuity at the behavioral level}

An essential matter of this study was, to understand sensory processing deficits of mice with different dysmyelination phenotypes, and correlate them to deficits observed in the auditory cortex. I used two different behavioral paradigms to test temporal acuity. One was the measurement of the inhibition of the acoustic startle reflex due to the presence of a gap in the background sound previous to the startle stimulus. The other paradigm addressed the same gap-detection mechanism, using a naturalistic behavioral testing environment (the AudioBox). For these tests, $\mathrm{MBP}^{\text {neo }}$ mice were used since they presented a strong dysmyelination phenotype without the motor abnormalities that prevented the $\mathrm{MBP}^{\text {shi }}$ from efficiently performing these tasks.

\section{Behavioral gap-detection using pre-pulse inhibition of the acoustic startle reflex}

Gap-elicited PPI of the ASR is a measure of sensorimotor gating, which refers to the filtering process made by a sensory system for higher-order processing (Iscru et al., 2009). Gapelicited PPI of the ASR is a widely used test in rodents to surface impairments in auditory temporal acuity (Dehmel et al., 2012; Popelář et al., 2017). In this test, I found a significant decrease in the PPI of the ASR in $\mathrm{MBP}^{\text {neo }}$ mice and a significant increase in their gapdetection threshold (see Figure 35, orange). These behavioral results confirm that $\mathrm{MBP}^{\text {neo }}$ mice show not only a reduction on temporal acuity in the auditory cortex at the electrophysiological level, but also a change in the perception of temporal properties of sounds.

\section{The severity of dysmyelination is relevant for temporal acuity}

One question that arose after the PPI experiment with $\mathrm{MBP}^{\text {neo }}$ mice was if a certain 'threshold' of myelin disturbances would account for the behavioral changes observed. I was wondering if a lower loss of myelin would be sufficient to generate similar phenotypes. For that reason I tested the heterozygote animals from the shiverer line $\left(\mathrm{MBP}^{+/}\right)$, which have $\sim 50 \%$ of reduction in MBP. Interestingly, I did not observe any differences between the $\mathrm{MBP}^{+/-}$and control animals regarding the PPI of the ASR (Figure 35), consistent with (Poggi et al., 2016). This suggests that a certain level (higher than $50 \%$ ) of myelin loss is needed to elicit a behavioral reduction of temporal acuity. Since it has been proposed that cortical activation is essential for gap detection (Leitner et al., 1993; Moreno-Paublete et al., 2017; Weible et al., $2014 a, 2014 b)$, I tested whether an only-cortical loss of myelin would affect the processing 
necessary for gap-detection. For that reason, I used the $\mathrm{MBP}^{\mathrm{emx}}$ mice which have severe forebrain-specific dysmyelination and observed no differences in the behavioral gap-detection (Figure 35). These results show that perceptual gap-detection is not affected by possible conduction blocks resulting from the lack of myelin of the responses entering the ACx and/or that gap-detection does not require the fine-tuning of timing of intra-cortical processes mediated by myelin.

In addition to the gap-detection threshold impairment, which suggests a defect in sensory gating, $\mathrm{MBP}^{\text {neo }}$ mice showed a reduction in the overall amplitude of the ASR (Figure 35). The gap-elicited PPI of the ASR involves the functionality of two circuits (Bullock et al., 1997; Koch, 1999): a sensory cortical circuit for gap detection (Moreno-Paublete et al., 2017) and a circuit of spinal, cranial and cerebellar neurons that must be coordinated in a short interval of time (Davis et al., 1982; Netrakanti et al., 2015). Lack of myelin resulting in desynchronization of these inputs would explain the reduced ASR. It has been shown that Purkinje cell degeneration (Parham et al., 2000) and cerebellar aspiration (Davis et al., 1982) can cause a reduction on the amplitude of the ASR without affecting PPI. This suggests that a reduction of the $\mathrm{ASR}$ in $\mathrm{MBP}^{\text {neo }}$ mice might be caused by a dysfunction in cerebellar circuits for rapid motor reflexes, in addition to the sensorimotor gating deficit. Both are processes disturbed by the lack of myelin.

\section{Behavioral gap-detection using the AudioBox}

Since the PPI gap detection is a reflex type response, I measured temporal acuity in the $\mathrm{MBP}^{\text {neo }}$ mice using a more naturalistic and complex behavioral paradigm. For that I used the AudioBox. Interestingly, I found a significant decrease of the visit number of $\mathrm{MBP}^{\text {neo }}$ mice on the first 4 days of habituation to the behavioral box. This behavior can be attributed to changes in novelty coping of these mutants. It is normal that upon exposure to a new environment, an organism is divided between exploring it or avoiding it (O'Keefe and Nadel). Low ambulatory behavior correlates to a fear response when an organism is exposed to a novel environment (Ramos and Mormède, 1998; Trullas and Skolnick, 1993). Previous studies have shown that $\mathrm{MBP}^{+/}$mice do not have changes in exploratory behavior or novelty preference (Poggi et al., 2016). This contrasts my results, which show that $\mathrm{MBP}^{\text {neo }}$ mice have reduced novelty coping, nevertheless, this behavior lasts for only a few days, probably the amount of time that it takes the $\mathrm{MBP}^{\text {neo }}$ mice to get familiarized with the environment. 
Overall, most importantly, I observed a reduction in gap-detection in $\mathrm{MBP}^{\text {neo }}$ mice. The gap detection deficit with the PPI paradigm was replicated using the AudioBox, indicating that it indeed reflected a perceptual deficit and not merely a reflex-related deficit. In general the results from the gap-detection task in the AudioBox yielded subtler effects than what I observed with the gap-PPI. This could be related to the difference in the amount of time the animals had to process the sounds, which was small in the PPI and long in the AudioBox. Consistent with this idea, $\mathrm{MBP}^{\text {neo }}$ mice spent longer time in the corner hearing the sound before nose-poking, compared to control animals. A parallel test that involved frequency discrimination (data not shown) yielded no differences in the $\mathrm{MBP}^{\text {neo }}$ mice compared to controls, suggesting that $\mathrm{MBP}^{\text {neo }}$ mice do not have a generalized learning or auditory problem, but very specific temporal-related deficits that correlate with the measured neuronal acuity in the auditory cortex.

\subsection{Conclusions}

The processing of auditory information can allow an organism different levels of cognition, from the fast response to a potentially dangerous stimulus, to the formation of an emotional association to a specific sound context. In this chapter, I studied the role of myelin mainly in temporal acuity and temporal reliability. As expected, delayed responses in auditory stations were observed. Additionally, I observed that along the auditory system, homeostatic mechanisms take place to compensate for desynchronized responses arising from dysmyelinated nerves. The fine-tuning of excitability of auditory neurons at higher-order stations is elegantly modulated by plastic changes in the action potential initiation site. Most importantly, both temporal acuity and reliability were impaired at the neuronal and perceptual level in mice with dysmyelination. The observed deficiencies, fall beyond the basic expected effects of reduced conduction velocity, since mechanistically, they appear related to secondary abnormalities to the loss of myelin. Auditory defects arising from developmental myelin loss seem related mainly to temporal aspects of sound processing. In humans adequate auditory temporal processing is essential for speech discrimination and the enjoyment of musical experiences. This study is the first of its class, to highlight the importance of myelin in higher-order auditory processing. 


\section{Chapter 2}

\section{Oligodendrocyte metabolic impairments affect axonal function}

\subsection{Introduction}

\subsubsection{Oligodendrocyte metabolic support}

Axons have high energy demands. They need to maintain an uniform distribution of cytoskeletal proteins, to extend energy supply along the axon, to maintain calcium homeostasis, to prevent the generation aberrant protein aggregates, and to protect themselves against oxidative stress (Beirowski, 2013). Because of their length and metabolic demand, they are prone to injury. The close proximity of oligodendrocytes to axons not only provides them with isolation for speeding up conduction velocity, but also with support in moments of high metabolic needs (e.g. at high firing rates). It has been shown that myelin perturbations can cause axonal pathologies in absence of an overt de/dysmyelination phenotypes (Edgar et al., 2009; Griffiths et al., 1998; Lappe-Siefke et al., 2003; Werner et al., 2013) suggesting a role of the oligodendrocytes beyond myelination (Spencer et al., 1979).

Oligodendrocytes can metabolically support axons using mechanisms that are independent of myelination (Fünfschilling et al., 2012; Lee et al., 2012; Morrison et al., 2013) (see Figure 37). These mechanisms are currently not well understood. Myelinating 
oligodendrocytes perform glycolysis, producing monocarboxylates (i.e. lactate, pyruvate and ketone bodies). Monocarboxylates are important for energetic metabolism and are rapidly transported across plasma membranes through monocarboxilate transporters (MCT) into the axon. In the axon, they can be used by the axonal mitochondria to generate ATP (Fünfschilling et al., 2012). Since myelin isolates axons from the environment, lactate/pyruvate transport might be an essential mechanism when other sources of energy are scarce (Bercury and Macklin, 2015).

MCTs are a family of 14 proteins encoded by the SLC16A gene family (PérezEscuredo et al., 2016). The best characterized MCTs are MCT1-4, proton-linked transporters, which have 12 transmembrane domains, intracellular $\mathrm{C}$ - and N-termini and a large cytosolic loop in between the transmembrane domains (Halestrap, 2012). MCTs are expressed in a great variety of tissues and have been implicated in several physiological functions (PérezEscuredo et al., 2016). In the CNS, different MCTs are expressed in different cell types: MCT2 is only expressed in neurons, while MCT1 is expressed mainly in oligodendrocytes, and MCT4 in astrocytes (Domènech-Estévez et al., 2015; Lee et al., 2012). The loss of $~ 50 \%$ of the MCT1 significantly affects the maintenance of myelinated axons in the long term (Lee et al., 2012).

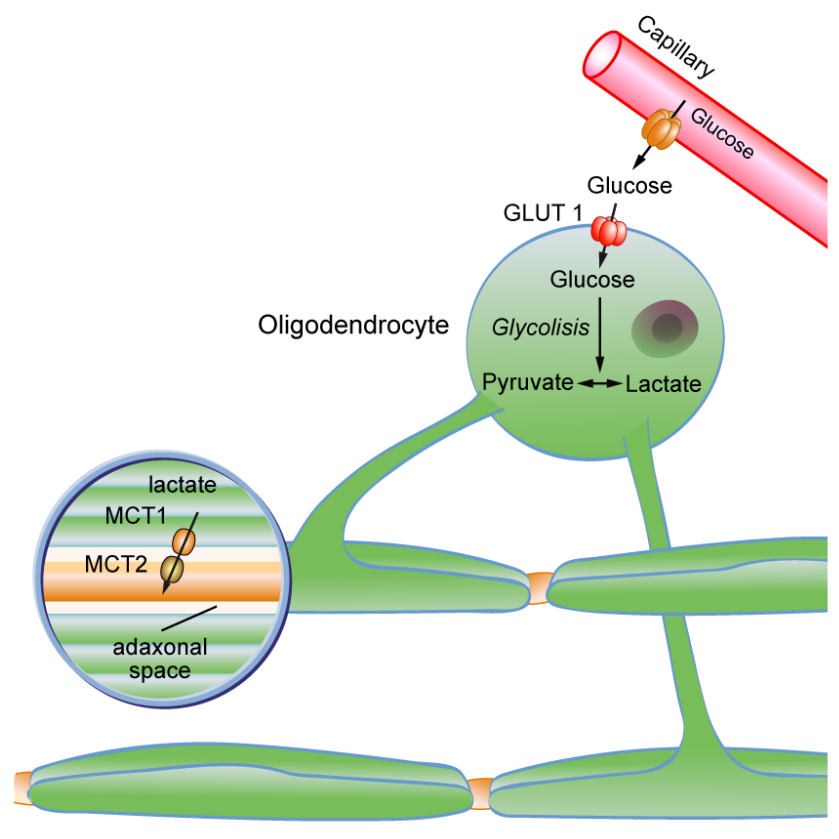

Figure 37. Schematic representation of the metabolic support from oligodendrocytes to axons using lactate.

An oligodendrocyte can uptake glucose from the bloodstream trough GLUT1 transporters. The glucose is then used for glycolysis, which generates as byproducts lactate and pyruvate. A blow-up on the axo-glial contact shows that lactate can then be transported from the innermost myelin membrane to the axon via MCT1 (oligodendrocytes) and MCT2 (axons) transporters. Axons can then use this lactate. Image credit: Jan Ficner. 


\subsubsection{The axo-myelinic synapse}

For the efficient transfer of metabolites to axons, it is necessary to have a communication line between the axons and the oligodendrocytes. An 'axo-myelinic' synapse (AMS) has been proposed as a signaling mechanism from the axon to the oligodendrocyte (Micu et al., 2018; Stys, 2011). Myelinic 'nanochannels' are proposed to be connecting the adaxonal space to the oligodendrocyte's cytoplasm, forming a communication route (Micu et al., 2018). The AMS is composed of comparable structures to the typical synapse: the adaxonal space (resembling synaptic cleft), the axonal receptors (pre-synaptic receptors), the inner myelin loop (post-synapsis), neurotransmitter release, transporters and oligodendrocyte receptors (post-synaptic receptors). In addition, the internodal area of the axon is polarized $\left(\mathrm{V}_{\mathrm{Rm}}=\right.$ $80 \mathrm{mV}$ ) and it has been proposed that a protein (plasmolipin) located within compact myelin and the axolemma is responsible for the polarization of the myelin sheath, like the postsynaptic membrane is (Stys, 2011). In addition, a $\mathrm{Ca}^{2+}$ buffering system was found in myelin ( $\mathrm{Ca}^{2+}$-ATPase) (Stys, 2011). Active signaling between white matter axons and the oligodendrocytes was evidenced in myelin $\mathrm{Ca}^{2+}$ fluctuations upon vesicular glutamate release from stimulated axons (Micu et al., 2016), proving the functionality of the AMS.

Impaired communication between oligodendrocytes and axons leads to pathologies. It has been suggested that some MS lesions, especially the ones with low inflammatory profiles, could result from perturbations at the AMS. In this case, accumulation of axonal mitochondria and enlarged inner-tongues were observed, suggesting the involvement of metabolic impairments probably due to altered glutamatergic transmission (Micu et al., 2018). Similar observations were made for other neurodegenerative, neurodevelopmental, and psychiatric disorders (for review, see (Micu et al., 2018). Thus the AMS may be susceptible to injury, and its study could uncover further roles for oligodendrocytes in the maintenance of proper brain function.

\subsubsection{Other forms of oligodendrocyte metabolic support}

The role of oligodendrocytes in metabolically supporting axons not only relies on a direct distribution of metabolites. A recent study shows that there is a non-linear dependence of energy consumption along axons, and that energy consumption depends on axonal branching, volume, and channel distribution. In addition, the authors proposed a model in which AP propagation requires 15\% more energy at the axon initial segment (Ju et al., 2016), which suggests that different axonal domains have distinct metabolic needs. Supporting 
evidence has been found in the fact that mitochondria accumulate at axonal regions with the highest energy demands (Misko et al., 2012), such as the distal part of the axon initial segment (AIS) (Li et al., 2004), at unmyelinated portions (Minckler et al., 1976), at nodes of Ranvier (nR) (Berthold et al., 1993) and at presynapses (Misgeld et al., 2007). It has been suggested that the localization of these mitochondria depends on axo-glial contacts since a disruption of the paranodal contacts generates the accumulation of aberrant mitochondria close to the $\mathrm{nR}$ (Einheber et al., 2006). This could be mediated through a direct signaling mechanism for the mitochondria to become static at excitable axonal domains, or through secondary mechanisms, like local alterations in axonal transport or physical properties of axons as they become constricted at these areas, creating a physical barrier for mitochondria to flow (Einheber et al., 2006; Li et al., 2004).

Additional mechanisms of oligodendrocyte metabolic support of axonal function can result from the communication between these cell types with astrocytes through gap junctions (Nualart-Marti et al., 2013). White matter astrocytes are able to support trough glycogen release the function of axons during periods of high frequency stimulation or aglycemia (Brown et al., 2003; Wender et al., 2000). It has been proposed that lactate transfer happens from astrocytes to oligodendrocytes, and in turn, to the axons and that the axo-myelinic synapse signaling reaches the astrocytes (Stys, 2011).

These observations highlight the importance of the study of glial cells in normal brain function and disease. The recently described novel roles of these cells in neuronal processing represents, to a certain extent, that they were understudied in the past since they were thought as passive space-filling cells. Nowadays, we face ourselves with the necessity of widening the 'neuro' in neurosciences and give them the place they deserve. Studies for the dynamic assessment of neuronal and glial function combined are very scarce in the field. In comparison to Chapter 1 , in this chapter I studied axonal cortical auditory function in mutant mice that do not show a strong dysmyelination phenotype, but have either reduced metabolic support to the axons or an internal metabolic dysfunction due to oligodendrocyte-specific glycolytic impairment. 


\subsection{Results}

\subsubsection{Reduced oligodendrocytic trophic support does not impair basic auditory processing in the brainstem}

In general, glial cells seem to be playing an important role in the support of axons. After observing temporal auditory abnormalities in mice with different degrees of dysmyelination, my next question was whether mice with a reduction in the metabolic axo-glia coupling would affect auditory processing at different levels. For this reason, I used the MCT1 ${ }^{+/}$mice (Lee et al., 2012), a mouse model with a reduction in the MCT1 protein of $\sim 50 \%$. While this transporter is located in oligodendrocytes and astrocytes (Domènech-Estévez et al., 2015) it is believed to play a more crucial role in oligodendrocytes because of the close contact these cells have with axons and the trophic support hypothesis (see section 2.1.1). These mice present a reduction in the transport of lactate from the glia to the axons, which impairs, in the long term, axonal stability (Lee et al., 2012). A second mouse model (the Hif $1 \alpha^{f / / f / t} ; \mathrm{PLP}$ creRT2 $^{+}$) was used to address the question of whether reduced metabolism in oligodendrocytes would impair axonal function. My goal was to study auditory cortex (ACx) function in both glial-metabolic dysfunctions.

\section{A brief introduction to the Hif1 $\alpha$ model}

The Hif1 $\alpha^{f / f t} ;$ PLP-creRT2 ${ }^{+}$mice were obtained as described in Materials and Methods (section II.I.IV) from Iva Tzvetanova. Briefly, these mice have an oligodendrocyte-specific inducible reduction of the hypoxia inducible factor $1 \alpha(\mathrm{HIF} 1 \alpha)$. The hypoxia inducible factors are transcriptional regulators that under low $\mathrm{O}_{2} /$ glucose conditions, mediate coping responses to hypoxia (Aragonés et al., 2009). The HIF1 $\alpha$ is a key regulator of the activation of the glycolytic pathway together with the inhibition of mitochondrial respiration (Majmundar et al., 2010; Zhong and Mostoslavsky, 2010), promoting the accumulation of lactate/pyruvate for export. It is likely that HIF1 $\alpha$ would be a major modulator of oligodendrocyte glycolysis and the metabolic support of axons. For the selective inactivation of this factor in oligodendrocytes in an inducible manner, mice expressing PLP-creERT2 were crossed with others expressing the HIF1 $\alpha^{f / f t / l}$. Both control (Hif1 $\alpha^{f / f t} ;$ PLP-creRT2 ${ }^{\omega t}$ ) and mutant mice (Hif1 $\alpha^{f / f t} ;$ PLP-creRT2 ${ }^{+}$) received tamoxifen injections as explained in Materials and Methods (section II.I.IV) at 8 weeks of age to avoid interfering with developmental myelination processes. Mice were then tested for auditory function at $\sim 13$ weeks of age. A brief description of experimental observations made by Iva Tzvetanova will be presented here, without the data being shown. 
Hif $1 \alpha^{f / f f} ;$ PLP-creRT2 ${ }^{+}$mice have reduced mRNA expression of glycolytic enzymes in the corpus callosum, confirming that HIF1 $\alpha$ is important for glycolysis in oligodendrocytes. At $\sim 6$ weeks post-tamoxifen injection (around the age of auditory testing), axons showed, a reduction at the mRNA level of glycolytic enzymes; at the EM level, abnormal mitochondria (increased in size) and enlarged inner tongues (a typical phenotype of metabolically challenged axons). A mild increase in the g-ratios measured from the corpus callosum, shows a slight reduction in myelin thickness. Axonal pathology becomes evident in aged animals and precedes neuro-inflammation. Optic nerves from the Hif $1 \alpha^{f / f f l}$;PLP-creRT2 ${ }^{+}$mice show reduced ability to cope with glucose deprivation. Hif1 $\alpha$ is confirmed as an important regulator of oligodendrocyte glycolysis and its impairment impacts axonal stability, probably due to a reduction of metabolic support.

\section{Subcortical auditory processing is not impaired}

As discussed in Chapter 1, ABRs were measured from the $\mathrm{MCT}^{+/-}$mice to understand the basic functioning of subcortical auditory processing: response amplitudes, thresholds, and latencies. $\mathrm{ABR}$ shape of $\mathrm{MCT}^{+/-}$mice was no different from control animals (Figure 38-A). Sound detection threshold was around $35 \mathrm{~dB}$ for both groups (Figure 38-B) without a significant difference $(p=0.39)$. No latency differences were observed in any of the waves tested using click stimuli at $80 \mathrm{~dB}(p=0.84, p=0.92, p=0.18, p=0.97, p=0.23$ respectively for peaks I to V). A small reduction in amplitude was observed in the $\mathrm{MCT} 1^{+/-}$mice only seen in wave III ( $p=0.34, p=0.29, p=0.01, p=0.15, p=0.22$ for waves I to $V$ respectively). This reduction in amplitude was very mild and not persistent trough other ABR waves. In addition, no increase in gain was seen at latter stations. In general, it seems that a $50 \%$ reduction of the MCT1 protein in glial cells does not impair auditory brainstem responses severely.

As for the Hif $1 \alpha^{f / f / f l}$;PLP-creRT2 ${ }^{+}$mice, a small number of animals was tested $(\mathrm{n}=2$ for each group), and preliminary observations suggest that, similar to what was observed with the $\mathrm{MCT}^{+/-}$mice, Hif1 $\alpha^{f / / f l} ; \mathrm{PLP}-\mathrm{CreRT}^{+}$animals do not have strong subcortical auditory (data not shown). 
A

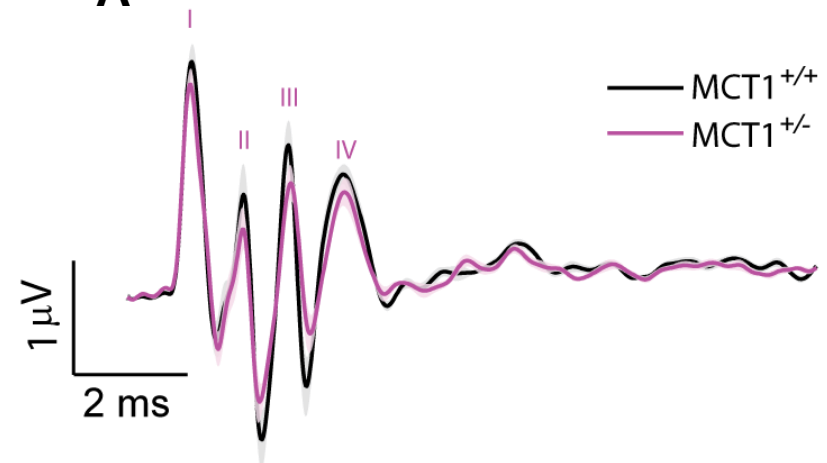

B

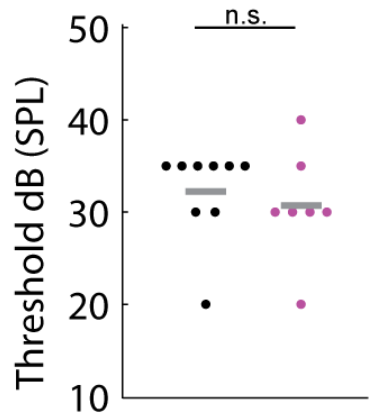

C

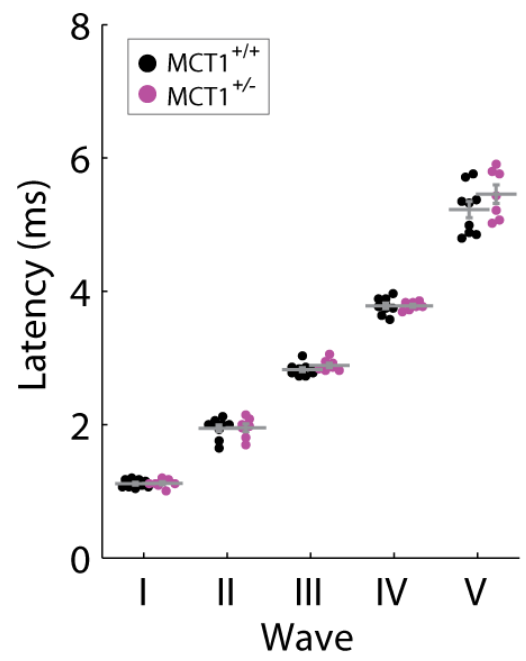

D

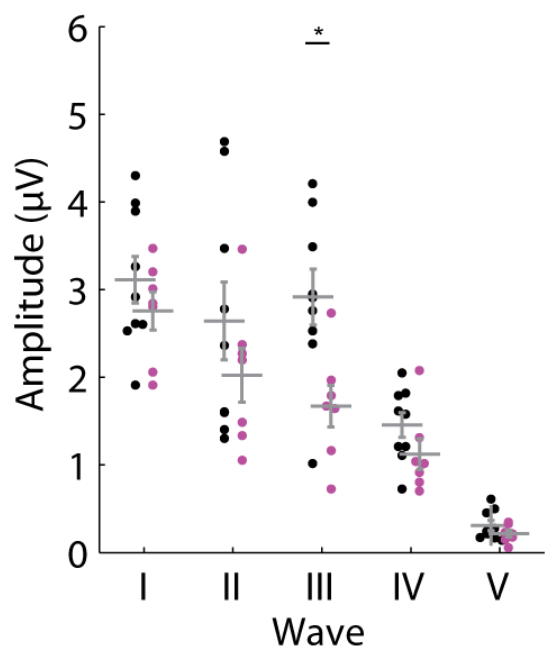

Figure 38. Subcortical auditory processing is not impaired in $\mathrm{MCT} 1^{+/}$mice.

A) The 5 characteristic waves of the ABRs, belonging to 5 different auditory stations are appreciated. Waves represent responses from the auditory nerve (I) until the inferior colliculus (V) for $\mathrm{MCT}^{+/+}$(black) and $\mathrm{MCT} 1^{+/-}$(purple) animals. $\mathrm{MCT}^{+/-}$show no delays in auditory potentials elicited by clicks at $80 \mathrm{~dB}$. Normal shape of the ABR is appreciated in mutant animals. B) No differences are seen at the threshold level $(p=0.39)$, and indirect measure of outer hair cell function. Gray bars show the mean per group. C) None of the waves show a significant delay in $\mathrm{MCT} 1^{+/}$ animals, compared to control. D) $\mathrm{MCT} 1^{+/}$mice have a reduction in wave III amplitude (ANOVA, $p=0.01$ ). All grey bars show the mean and S.E.M. per group. $n=9 \mathrm{MCT} 1^{+/+}$and $n=7 \mathrm{MCT}^{+/-}$animals. 
The characterization of response amplitudes and latencies in the ACx was carried out for both $\mathrm{MCT}^{+/}$and Hif1 $\alpha^{\text {fl/fl }}$;PLP-CreRT2 ${ }^{+}$. In Figure 39 we can see the effects in basic auditory processing in the ACx, of impairment in metabolic transport from the glia (Figure 39A to $\mathrm{C}$ ) or due to a metabolic defect in oligodendrocytes that would probably, as a secondary effect, reduce the metabolic support to the axons (Figure 39-D to E). MCT1 ${ }^{+/}$mice have a strong reduction in response amplitude in response to clicks, as seen in Figure 39-A, nevertheless, quantification of response amplitude did not yield a significant difference $(p=0.17)$.
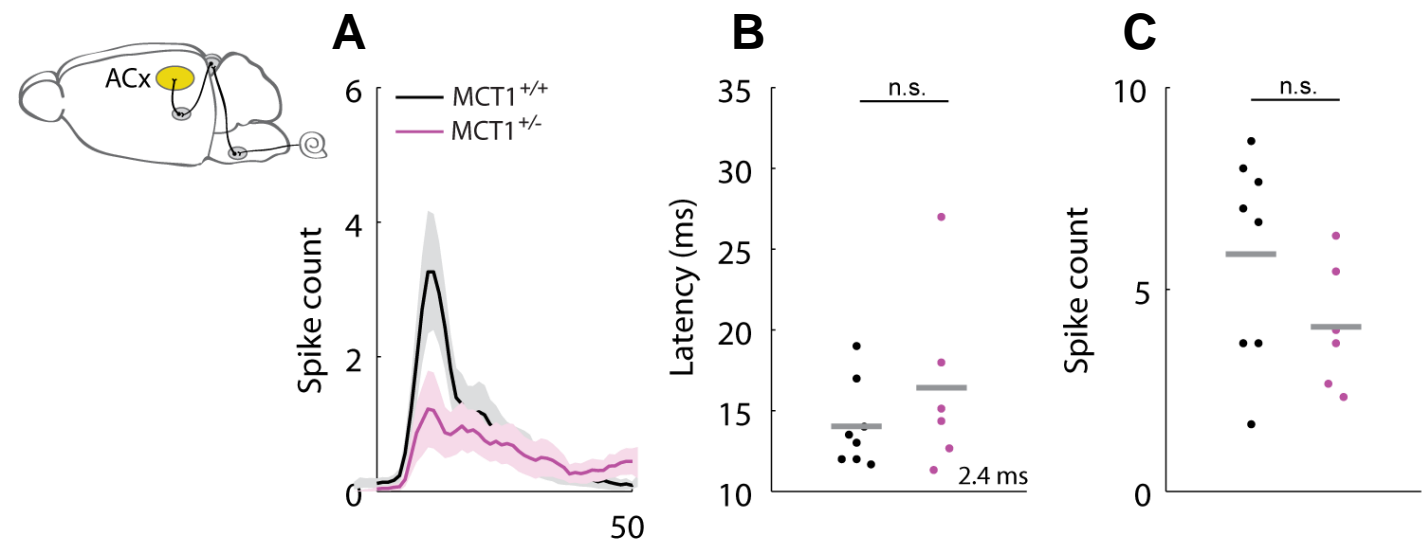

Time (ms)
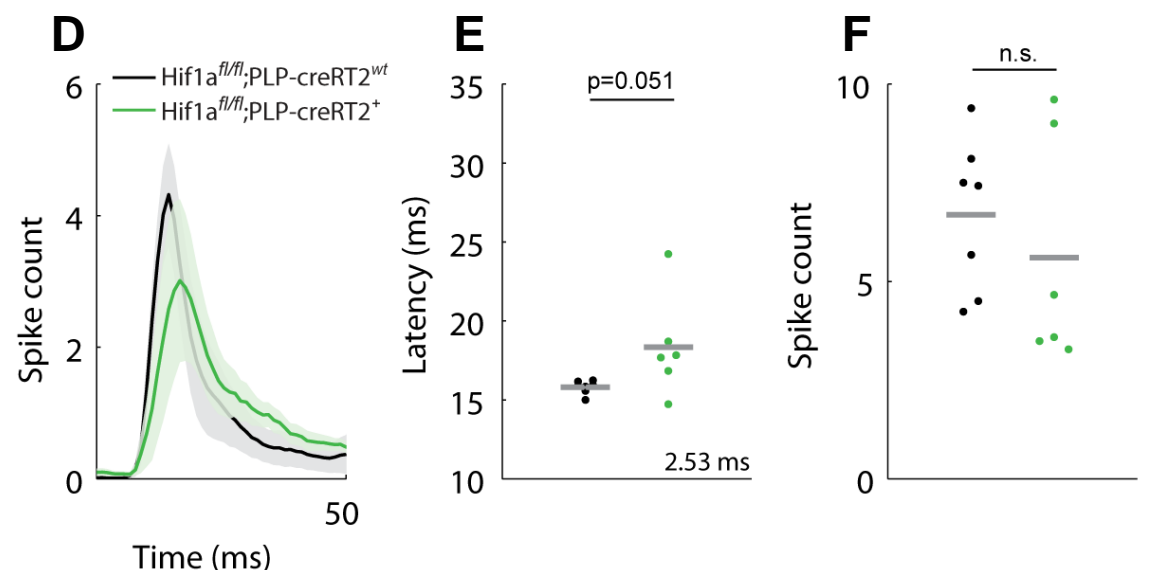

Time (ms)

Figure 39. Auditory cortex responses in $\mathrm{MCT}^{+/-}$and $\mathrm{Hif} 1 \alpha^{\mathrm{fl} / \mathrm{fl}} ; \mathrm{PLP}-\mathrm{CreERT2}{ }^{+}$mice.

A) PSTH of the auditory cortex response to a click stimulus for control (black) and $\mathrm{MCT} 1^{+/-}$(purple) mice. No differences in latency are obvious. B) Quantification of response latencies. No differences were seen between control (black dots) and mutant animals (purple dots) $(p=0.47)$. The number in the right lower corner depicts the differences between control and mutant means. Grey bars depict the mean per group. C) Response amplitudes in the ACx. No significant differences are seen between groups ( $p=0.17)$. D) Same as in $A)$ for the Hif1 $\alpha^{\mathrm{fl} / \mathrm{f}} ;$ PLP-CreERT2 ${ }^{+}$mice. E) Latency quantification reveals a slight shift in the $\mathrm{Hif1} \alpha^{f / / f l} ; \mathrm{PLP}-\mathrm{CreERT2}{ }^{+}$mice $(\mathrm{p}=0.051)$. F) Amplitude quantification. No significant differences are seen for the Hif1 $\alpha^{f / / f 1} ; P_{L P}-C r e E R T 2^{+}$mice $(p=0.44) . n=8 \mathrm{MCT}^{+/+}$and $n=8$

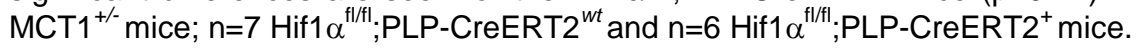


Since this effect was also seen with other sound protocols, I conclude that MCT1 ${ }^{+-}$ mice do have a reduction in response amplitude, but more data is necessary to confirm this effect. $\mathrm{MCT} 1^{+/}$mutants did not show a significant increase in latency (Figure 39-B) $(p=0.47)$ at the age of testing. This suggests that a defect in the transport of monocarboxilates from the glia to the axons does not impair conduction severely in the ACx. Nevertheless, in the Hif $1 \alpha^{\mathrm{fl} / f \mid} ; \mathrm{PLP}-\mathrm{CreERT2}{ }^{+}$, there is a tendency of a response latency increase in the ACx, which is already observable at the PSTH level. This effect was borderline significant $(p=0.051)$ (Figure 39-D and E). In addition, no changes in response amplitude are seen in Hif $1 \alpha^{\text {fl/fl. }}$; PLPCreERT2 ${ }^{+}$mice $(p=0.44)$ (Figure 39-E).

Impaired axo-glial support in $\mathrm{MCT} 1^{+/-}$does not affect the latency of sensory evoked responses, and there was a strong tendency towards a reduction in response amplitude. Defective oligodendrocyte metabolism in the Hif $1 \alpha^{f / f / f} ; P_{\text {LP-CreERT2 }}{ }^{+}$mice generates a subtle response latency increase in the ACx. Overall, axons that live under a restricted metabolic environment might show differences in their firing patterns, with tendencies towards increased latencies and reduced response amplitude. I then focused in auditory protocols that would be more challenging for the neurons to follow.

\subsubsection{An oligodendrocyte-specific metabolic defect impairs temporal processing in the $\mathrm{ACx}$}

To understand if defects in temporal processing seen with dysmyelination are related purely to conduction velocity deficits or are also related to an axonal inability to respond to short variations on the stimulus timing, I used the same protocols for temporal assessment seen in Chapter 1 (gap-detection and click tracing), for the metabolic mutants.

In Figure 40, we can see the results of the gap-detection assessment in the $\mathrm{MCT} 1^{+-}$ mice. Individual examples of control and mutant animals (Figure 40-A) illustrate that mutant animals appear to have reduced spiking probability to small gaps compared to control animals. In addition, $\mathrm{MCT}^{+/}$mice had strongly reduced response amplitudes, especially when looking at the responses to broad-band noise (Figure 40-B). This was also reflected in the pre-gap sound, where I found a significant reduction in the response amplitude in mutant animals (ANOVA, $F(1,252)=30.06, p<0.0001$ ). This reduction was also maintained through all the gap lengths ( $p<0.001$ for all gaps). Because of the amplitude difference between control 
and mutants, the comparison of post-gap windows between groups needs to take differences in amplitude into consideration. Indeed, the comparison of the PSTH at different gaps, showed a significant effect of group (ANOVA, $F(1,1612)=167.29, p<0.0001$ ) and a significant effect of gap $(F(5,1612)=4.69, p=0.0003)$ but no interaction $(F(5,1612)=1.87, p<0.096)$ (Figure 40-B). The reduction in amplitude seems to be a generalized feature of the cortical responses in $\mathrm{MCT}^{+/}$mice, since a tendency towards a lower amplitude was also observed in click responses (Figure 39).

A
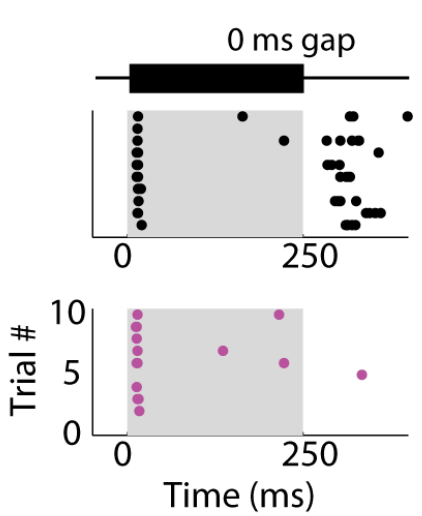

B

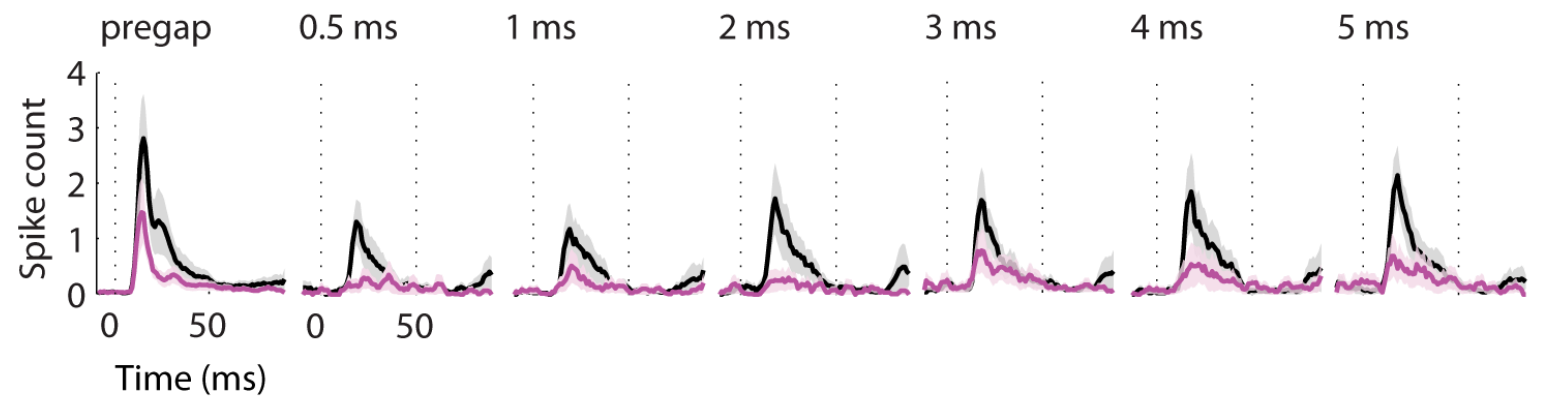

Figure 40. Temporal acuity is not impaired in the auditory cortex of $\mathrm{MCT1}^{+/-}$mice.

A) An individual example of the gap-detection recordings of a control (upper, black) and mutant (lower, purple) mouse. These raster plots represent the spiking activity (individual dots) that occurs during sound presentation (gray patches) to the 10 stimulus repetitions. Above the raster plots, a representation of the sounds played is presented in black bars, for a sound without a gap (0 ms gap, left side) and a sound with a 2 ms gap (right side). B) The average PSTH of all animals of control (black) and $\mathrm{MCT}^{+/-}$mice (purple) with the S.E.M. represented as a shaded error bar following the same color code. Pre-gap and post-gap responses to the presentation of 0.5 to 5 $\mathrm{ms}$ are represented. Significant differences were seen between groups for the PSTH comparison throughout the gaps (ANOVA, $p<0.0001$ ). $n=8$ control and $n=6$ mutant mice. C) Quantification of the differences between baseline and post-gap activity (50 ms window). A dotted black line depicts the threshold of $p=0.05$ for significance. The longer the gap length, the higher the significance value. No significant differences were seen between groups (Kruskal-Wallis test, $p=0$. 4). The plot shows the median values for both groups, error bars represent the standard error of the median. The internal distribution plot in $\mathrm{C}$ ) shows all the $p$-value distributions for the $3 \mathrm{~ms}$ gap condition in control (gray) and mutant (purple) mice. A vertical black dotted line depicts the $p$ value corresponding to 0.05 significant. It is possible to see that most of the responses of both the control and mutant to the $3 \mathrm{~ms}$ gap are significantly different than baseline activity (left side of the vertical line) and have very similar distributions. $n=13$ recording sites from 8 control animals and $n=8-9$ recordings from $6 \mathrm{MCT}^{+/-}$mice. 
To overcome the confounding effect of the difference in amplitude when interpreting the post-gap responses, instead of comparing one group with another, I tested whether a significant evoked response was observed after each gap, comparing it to the baseline activity for individual recordings as in section 1.2.3. No differences in baseline activity were seen between groups $(p=0.56)$. Interestingly, I did not observe an effect of gap in the reduction of $p$-values (Kruskal-Wallis test, $x^{2}(9, n=219)=9.31, p=0.40$ ), probably due to the strong responses of control animals to the $0.5 \mathrm{~ms}$ sound. $\mathrm{MCT}^{+/-}$mice have normal temporal acuity.

Individual examples of a Hif1 $\alpha^{\mathrm{fl} / \mathrm{fl}} ; \mathrm{PLP}-\mathrm{CreERT}{ }^{\mathrm{wt}}$ and a Hif $1 \alpha^{\mathrm{fl} / \mathrm{fl} /} ; \mathrm{PLP}-\mathrm{CreERT2}{ }^{+}$ recording are shown in Figure 41-A, for a sound without a gap and one with a 2 ms gap. The post-gap PSTH at different gap lengths (Figure 41-B) shows that the amplitude of pre-gap responses in Hif $1 \alpha^{\text {fl/flit:PLP-CreERT2 }}{ }^{+}$is significantly different from control animals (ANOVA, $F(1,189)=21.31, p<0.0001)$.

Because of this difference, the comparison between control and mutant animals is hard to interpret, as discussed with the MCT1 mice. In Figure 41-C, we can see the comparison of baseline activity with post-gap activity. As expected, the longer gaps elicited stronger spiking probability, both in control and mutant animals. I observed a significant effect of gap overall, (Kruskal-Wallis test, $x^{2}(9, n=221)=35.25, p=5.3 \mathrm{e}^{-5}$ ), and a significant effect of group (Kruskal-Wallis test, $\left.x^{2}(1, n=221)=22.8, p=1.7 e^{-6}\right)$. There were no differences in baseline activity between groups $(p=0.57)$. These results show that, similar to what was observed for MBP ${ }^{\text {shi }}$ mice, the Hif $1 \alpha^{\text {ff/fl }}$;PLP-CreERT2 $2^{+}$mice have a strong reduction in spiking probability specially after the presentation of 1,2 and $3 \mathrm{~ms}$ gaps $(\mathrm{p}=0.01,0.058$ and 0.013 ). This reflects a strong impairment in temporal acuity of mutant mice with an oligodendrocyte metabolic impairment. Interestingly, the effect in these mutants, for which myelin thickness is only mildly impaired, is comparable to that seen in $\mathrm{MBP}^{\text {shi }}$ mice with extensive dysmyelination. 
A
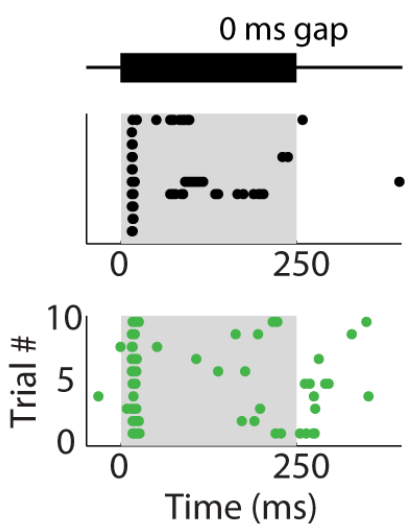

Time (ms)

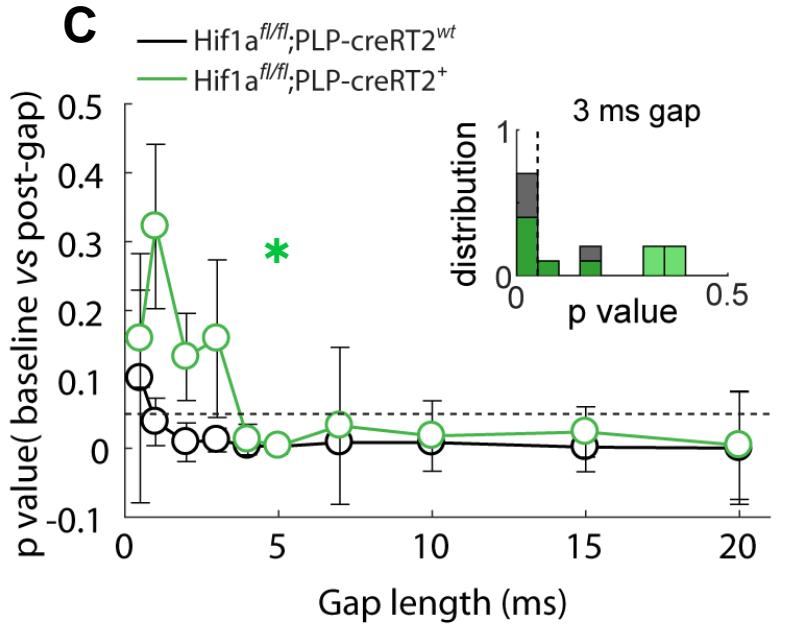

Gap length (ms)

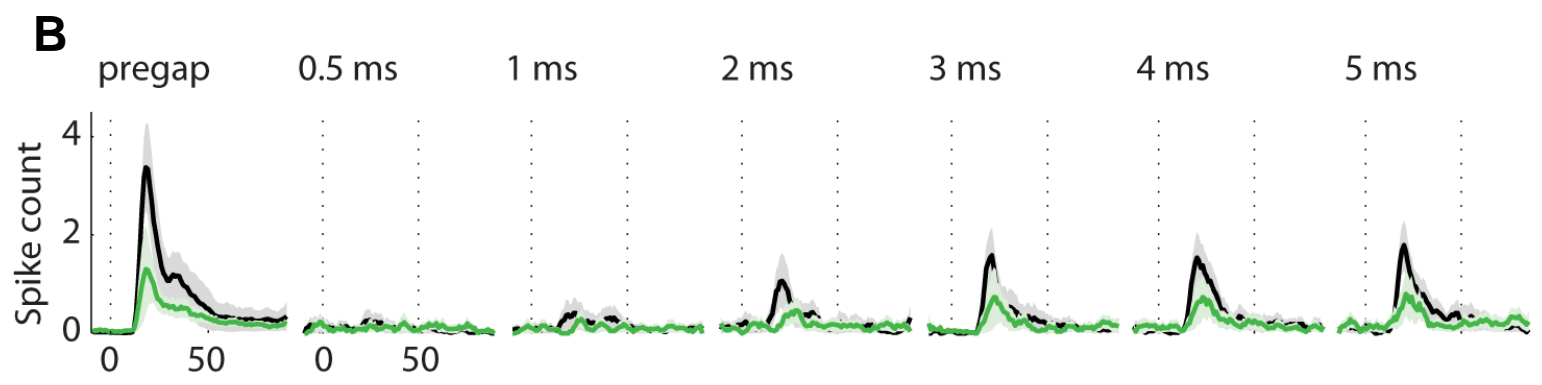

Time (ms)

Figure 41. Temporal acuity is impaired in the auditory cortex of Hif1 $\mathrm{a}^{n /} \mathrm{f} ; \mathrm{PLP}-\mathrm{creRT2}{ }^{+}$mice.

A) An individual example of the gap-detection recordings of a control (upper, black) and mutant (lower, green) mouse. These raster plots represent the spiking activity (individual dots) that occurs during sound presentation (gray patches) to the 10 stimulus repetitions. Above the raster plots, a representation of the sounds played is presented in black bars, for a sound without a gap (0 ms gap, left side) and a sound with a 2 ms gap (right side). B) The average PSTH of all animals shows the responses of control (black) and Hif1a $a^{f / f} ; P^{\prime}$ LP-creRT2 ${ }^{+}$mice (green) with the S.E.M. represented as a shaded error bar following the same color code. Pre-gap and post-gap responses to the presentation of 0.5 to $5 \mathrm{~ms}$ are showed. Significant differences were seen between groups throughout the gaps (ANOVA, $p<0.0001$ ). $n=6$ control and $n=6$ mutant mice. C) Quantification of the differences between baseline and post-gap activity ( $50 \mathrm{~ms}$ window). A dotted black line depicts the threshold of $p=0.05$ for significance. The longer the gap length, the higher the significance value. A significant difference was seen for gaps (ANOVA, $p<0.0001$ ) and groups (ANOVA, $p<0.0001$, green asterisk). The plot shows the median values for both groups, error bars represent the standard error of the median. The internal distribution plot in $\mathrm{C}$ ) shows all the $\mathrm{p}$-value distributions for the $3 \mathrm{~ms}$ gap condition in control (gray) and mutant (green) mice. A vertical black dotted line depicts the $p$ value corresponding to 0.05 significance. Most of the responses of both the control and mutant to the $3 \mathrm{~ms}$ gap are significantly different than baseline activity (left side of the vertical line) and have very similar distributions. $n=8-13$ recording sites of 6 control and $n=10-12$ recordings of 6 mutant mice. 


\subsubsection{An oligodendrocyte-specific metabolic defect impairs rate coding in the ACx}

Rate coding was then assessed as part of the study of temporal resolution in $\mathrm{MCT} 1^{+/-}$mice. In Figure 42-A, we can observe an example raster-plot of an $\mathrm{MCT}^{+/+}$mouse and an $\mathrm{MCT} 1^{+/-}$ mouse to a set of 10 clicks at a rate of $2 \mathrm{~Hz}$. In these examples, the $\mathrm{MCT} 1^{+-}$mouse shows more variability in the spiking activity in the middle clicks (4 to 6) as compared to the control example. The mean of all recorded animals was plotted per group (Figure 42-B) and showed as a blowup of only the first, fifth and last click for a repetition rate of 5 and $10 \mathrm{~Hz}$. As expected, the initial click response is strikingly lower in amplitude in the $\mathrm{MCT} 1^{+/}$mice, an effect that is conserved along the stimulus presentation.

Spike synchrony was measured as explained in section 1.2.3 and Materials and Methods section II.V.VI.VI. As expected, the percentage of spike synchrony is dependent on the stimulation rate (ANOVA, $F(4,339)=8.94, p<0.0001$ ) (Figure 42-C). Interestingly, I observed a significant difference between groups (ANOVA, $F(1,339)=11.59, p=0.0007$ ) but no interaction between the stimulation frequency and group (ANOVA, $F(4,339)=1.39, p<0.23$ ), and no effect of click number (ANOVA, $F(5,339)=0.23, p<0.94)$. Individual rate comparisons showed that the main effects were found at 2 and $10 \mathrm{~Hz}$ (ANOVA, $p=0.011, p=0.64, p=0.3$, $\mathrm{p}=0.087, \mathrm{p}=0.038$ for $2-10 \mathrm{~Hz}$ respectively). The reason why the difference between groups is observed at lower but not higher rates strives from the decay efficiency in the control group as rate increases. The $\mathrm{MCT} 1^{+/}$mice can respond to rates of $3 \mathrm{~Hz}$ and above as well as controls (with the exception of $10 \mathrm{~Hz}$ ), but while decreasing the rate below $3 \mathrm{~Hz}$ results in a linear increase in synchrony efficiency in the control group, the mutants cannot synchronize better than they do for $3 \mathrm{~Hz}$. These results show that $\mathrm{MCT}^{+/-}$mice present slight impairments in rate coding at low rates in the ACx, a much milder effect as that seen in $\mathrm{MBP}^{\text {shi }}$ mice. This means that having less routes where metabolic substrates can be exported from the oligodendrocytes, has a mild impact on axonal physiology, making it less prone to respond efficiently to continuous stimulation. 
A

B
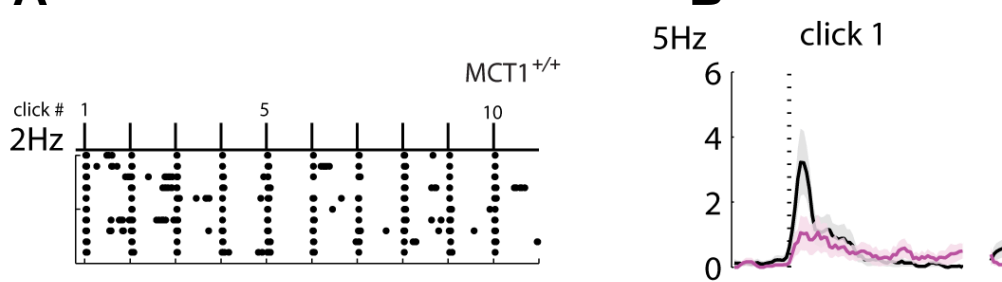

click 5

click 10 $10 \mathrm{~Hz}$
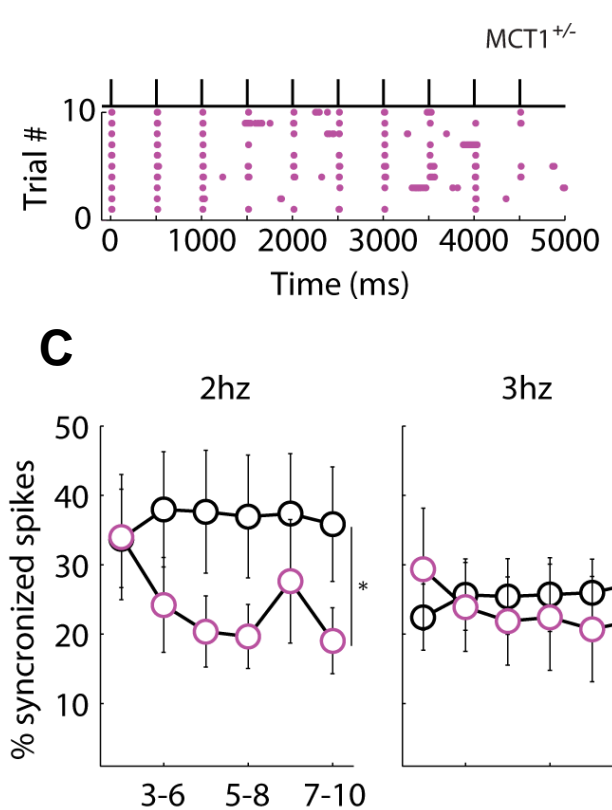

$3 \mathrm{hz}$
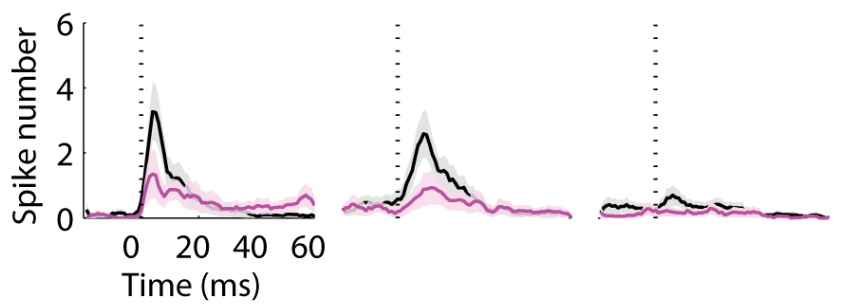

Sliding window

(click number)

Figure 42. Rate coding is slightly affected in the auditory cortex of $\mathrm{MCT}^{1 /-}$ mice.

A) An example raster plot of a control animal (upper, black) and a $\mathrm{MCT1}^{+/-}$mouse (lower, purple) in response to a set of 10 clicks depicted by the vertical bars above the raster plots. These examples correspond to the condition of $2 \mathrm{~Hz}$, in which each stimulus was $500 \mathrm{~ms}$ apart. Adaptation is observed, with lower spiking activity by the final clicks compared to the initial clicks. B) The mean PSTH of all animals recorded in control and mutant groups (conserving the same color-code). A blowup of click 1, 5 and 10 (as depicted in A) is shown. Thick lines show the mean PSTH of all animals recorded and the shaded area corresponds to the S.E.M. The upper panel shows the responses to a rate of $5 \mathrm{~Hz}$, and the lower to $10 \mathrm{~Hz}$. Vertical dotted lines represent stimulus onset. $\mathrm{n}=8$ control and $n=6$ mutant animals. C) The quantification of spike synchrony showed a significant difference between control and mutant mice (ANOVA, $\mathrm{p}<0.0007$ ) through the rates presented. Individual comparisons between groups considering all the clicks for each rate show that the strongest differences are at 2 and $10 \mathrm{~Hz}$ (ANOVA, $\mathrm{p}=0.011$ and $\mathrm{p}=0.038$ for 2 and $10 \mathrm{~Hz}$ respectively). $\mathrm{n}=8$ control animals and $\mathrm{n}=5-6$ mutant animals. 
While $\mathrm{MCT}^{+/-}$mice show mild deficits in rate coding, the study of the Hif $1 \alpha^{\mathrm{fl} / \mathrm{fl}}$;PLP-CreERT2 ${ }^{+}$ mice resulted in a more striking temporal deficit. In Figure 43-A we can see, similar to the observations in $\mathrm{MCT}^{+/-}$mice, that the main reliability deficits of the Hif1 $\alpha^{\mathrm{fl} / \mathrm{fl}}$;PLP-CreERT2 ${ }^{+}$ mice are observed in the responses to intermediate clicks. The mean of all recorded animals for the control group and the Hif $1 \alpha^{\mathrm{fl} / \mathrm{fl}}$;PLP-CreERT2 ${ }^{+}$mice, for the first, fifth and last click at a repetition rate of 5 and $10 \mathrm{~Hz}$ shows that the initial click response in mutants is similar to control animals, nevertheless, by the $5^{\text {th }}$ click, Hif $1 \alpha^{\mathrm{fl} / \mathrm{fl}} ; \mathrm{PLP}-\mathrm{CreERT2}{ }^{+}$mice show a strong decay in amplitude, an effect that is stronger at $10 \mathrm{~Hz}$ than at $5 \mathrm{~Hz}$ (see Figure 43-B, middle panels at click 5).

The measurement of spike synchrony confirmed the strong loss of reliability in Hif $1 \alpha^{\mathrm{fl} / f \mid} ; \mathrm{PLP}_{-C r e E R T 2}{ }^{+}$mice compared to control animals (ANOVA, $F(1,295)=47.46$, $p<0.0001$ ). A strong effect of stimulation rate was seen overall (ANOVA, $F(4,295)=31.84$, $p<0.0001$ ), together with a strong interaction between the stimulation frequency and group (ANOVA, $F(4,295)=4.43, p<0.0017$ ). No effect of click number was observed (ANOVA, $F(5,295)=0.07, p<0.99)$. Individual rate comparisons showed that the main effects were found at all stimulation rates lower than $20 \mathrm{~Hz}$ (ANOVA, $p=0.0071, p=0.0001, p<0.0001, p=0.036$, $\mathrm{p}=0.56$ for $5-20 \mathrm{~Hz}$ respectively). These results show that Hif $1 \alpha^{\mathrm{fl} / \mathrm{fl}} ; \mathrm{PLP}-\mathrm{CreERT2}{ }^{+}$mice, with a defect in oligodendrocyte glycolysis, present strong impairments in rate coding in the ACx, a much stronger phenotype than the one observed in $\mathrm{MBP}^{\text {shi }}$ mice. Since this mutation would not only be reducing the lactate shuttling to axons, but also impairing the metabolism of oligodendrocytes per se, it seems that having defective OLs generates stronger impairments in the axons, than just having a reduction in direct metabolic support.

Overall, these results show that an oligodendrocyte-specific metabolic impairment and the reduction of axo-glial metabolic support do not generate strong response latency changes in the auditory cortex. This might be attributed to the lack of a strong de/dysmyelination phenotype. Nevertheless, Hif1 $\alpha^{\mathrm{fl} / \mathrm{fl}} ; \mathrm{PLP}_{\mathrm{C}} \mathrm{CreERT2}^{+}$mice showed a borderline-significant increase in latency. Strikingly, both mutants showed a strong reduction in response amplitude to certain sounds, mainly broad-band noise. Auditory temporal defects were conserved in these mutants, similarly to what was seen with the dysmyelination models. Gap-detection was only impaired in the Hif1 $\alpha^{\mathrm{fl} / \mathrm{fl}}$;PLP-CreERT2 ${ }^{+}$and rate coding deficits were seen in both models, with the Hif1 $\alpha^{\mathrm{fl} / \mathrm{fl}} ; \mathrm{PLP}-\mathrm{CreERT2}{ }^{+}$being strongly affected. 
A

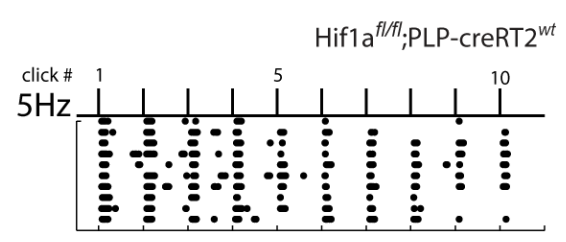

\section{B}

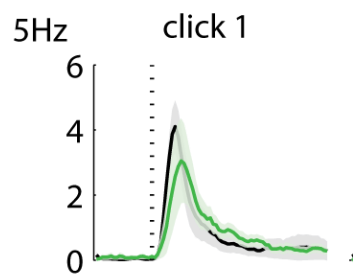

click 5

click 10

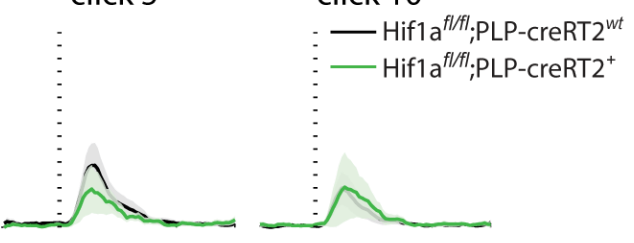

$10 \mathrm{~Hz}$
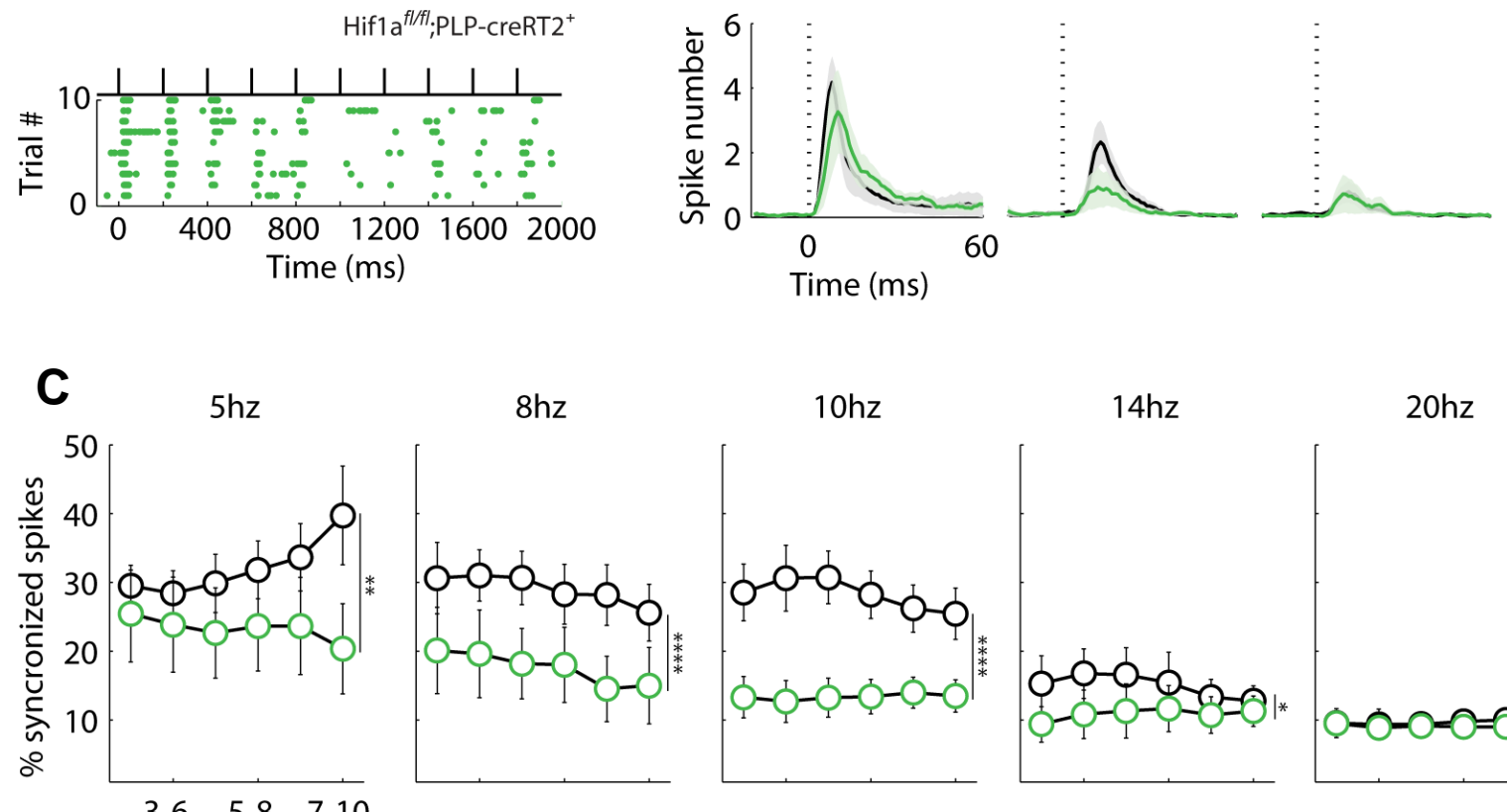

$8 \mathrm{hz}$

$10 \mathrm{hz}$

$14 \mathrm{hz}$

$20 \mathrm{hz}$
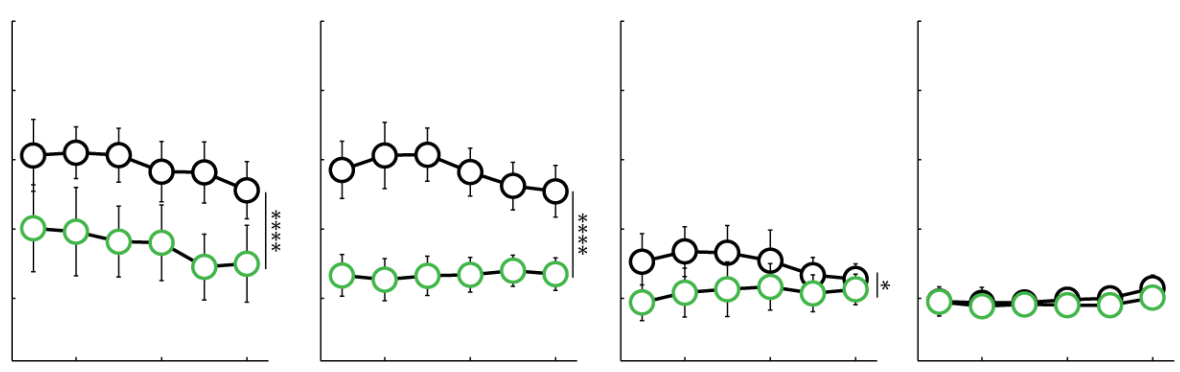

Sliding window

(click number)

Figure 43. Rate coding is affected in the auditory cortex of Hif $1 \alpha^{f / / f t} ; \mathrm{PLP}-\mathrm{CreERT}{ }^{+}$mice.

A) An example raster plot of a control animal (upper, black) and a Hif1 $\alpha^{\text {t/fft }}$;PLP-creERT2 ${ }^{+}$mouse (lower, green) in response to a set of 10 clicks depicted by the vertical bars above the raster plots. These examples correspond to the condition of $5 \mathrm{~Hz}$, in which each stimulus was $200 \mathrm{~ms}$ apart. Adaptation is observed, with lower spiking activity by the final clicks compared to the initial clicks. B) The mean PSTH of all animals recorded in control and mutant groups (conserving the same color-code). A blowup of click 1, 5 and 10 (as depicted in A) is shown. Thick lines show the mean PSTH of all animals recorded and the shaded area corresponds to the S.E.M. The upper panel shows the responses to a rate of $5 \mathrm{~Hz}$, while the lower to a rate of $10 \mathrm{~Hz}$. Vertical dotted lines represent stimulus onset. A reduction of response amplitude is seen in Hif $1 \alpha^{\text {t/ffit; }}$ PLP-creERT2 ${ }^{+}$mice in the middle clicks. $n=7$ control and $n=6$ mutant animals. C) The quantification of spike synchrony showed that there is a significant difference between control and mutant mice (ANOVA, $\mathrm{p}<0.0001$ ) through the rates presented. Individual comparisons between groups considering all the clicks for each rate show that the strongest differences are below $14 \mathrm{~Hz}$ (ANOVA, $p=0.0071, p=0.0001, p<0.0001, p=0.036$ for rates from $5-14 \mathrm{~Hz}$ respectively). $n=5-7$ control animals and $\mathrm{n}=5-6$ mutant animals. 


\subsection{Discussion}

It has been proven that oligodendrocytes can provide metabolic support for axonal function (Fünfschilling et al., 2012; Lee et al., 2012). In this chapter, my aim was to test whether the auditory abnormalities described in Chapter 1 can be explained through a reduction in oligodendrocyte metabolic support, resulting from the lack of tight contact between the oligodendrocytes and axons in the absence of myelin. With this question in mind, I used two different mice models of metabolic dysfunction: 1) the $\mathrm{MCT} 1^{+-}$mice, which have a reduction in the expression of the glial MCT1 transporter, and 2) the Hif $1 \alpha^{\text {ffftil }}$ PLP-creERT2 ${ }^{+}$mice, which lack the Hif1 $\alpha$ transcription factor in oligodendrocytes. Both models likely result in a restriction of lactate/pyruvate support. My main goal was to understand how these axo-glial metabolic impairments impact the stability of the axons in the cortex.

\section{Basic auditory processing}

Since the mutations in $\mathrm{MCT}^{+/-}$and Hif $1 \alpha^{f / f t /} ; \mathrm{PLP}-\mathrm{creERT} 2^{+}$mice are not affecting myelination per se, an increase in the response latencies was not expected. I observed that a $50 \%$ reduction of the expression of the MCT1 transporter does not alter the latency of auditory responses at the subcortical level (Figure 38) or in the cortex (Figure 39-B). In contrast, I observed a subtle response latency increase in the cortex of the Hif $1 \alpha^{f / f t /} ; \mathrm{PLP}-\mathrm{creERT} 2^{+}$mice (Figure 39-D). Preliminary ABR tests performed in these animals suggest that there are no peripheral impairments in these animals either (data not shown). A mild demyelinating effect, resulting in axons with thinner myelin, was observed at the age of testing in Hif $1 \alpha^{\text {ffffl }}$;PLPcreERT2 ${ }^{+}$mice (data not shown). This observation is surprising, since it suggests that metabolically impaired oligodendrocytes could be reducing their myelin sheaths to use them up as an energy source. In addition, this could explain the small effect in latency at the cortical level. In the case of the $\mathrm{MCT} 1^{+/}$mice, these animals also show demyelination but at much older ages (8 months) (Lee et al., 2012) compared to the Hif $1 \alpha^{f / f t / f} ; \mathrm{PLP}-\mathrm{creERT} 2^{+}$mice that were tested $\sim 13$ weeks of age.

One striking effect observed in these animals was that the spiking activity was severely dampened (Figure 42-B, Figure 40-B and Figure 41-B), which was reflected in the strong decrease in the PSTH amplitude. Especially the $\mathrm{MCT} 1^{+/}$model was accompanied by a persistent reduction in spiking probability. It has been suggested that during starvation, spontaneous firing can be maintained, to a certain extent, by monocarboxilates (Lutas et al., 
2014). Nevertheless, the effects of aglycemia must be very strong for the neurons to observe a reduction in spiking activity, a condition that I could not manipulate in vivo. This suggests that the reduction of metabolic support from oligodendrocytes to axons impairs spiking activity. This reduction is not due to a change in hearing threshold, since the ABR threshold measurement was normal in these animals (Figure 38-B). Another aspect that could be altering spiking activity is the level of spontaneous activity. It has been demonstrated that spontaneous activity in the cortex can impact stimulus-evoked responses, since the spiking probability is influenced by the ongoing variability of the network activity (Nguyen Trong et al., 2013; Schölvinck et al., 2012). In this case, the reduced spiking activity observed in $\mathrm{MCT}^{+/}$ and Hif1 $\alpha^{\text {fl/fl; }}$ PLP-creERT2 ${ }^{+}$mice was not due to changes in the spontaneous activity of cortical cells, since I saw no differences in baseline spiking between control and mutant mice in either mutant. The decrease in response amplitude was particularly evident when BBN was used as a stimulus. This suggests that both mutants have reduced spiking probability due to a defect in glial-metabolic support. The response amplitude when a click was used as a stimulus was only impaired in the $\mathrm{MCT} 1^{+/}$mice. The basic explanation for this difference is that the click stimulus is physically very different from more complex sounds. A click is a very short upward increase in pressure (Schnupp et al., 2012) eliciting sharp onset responses, which would generate stronger spike synchronization and larger PSTHs because more spikes would be clustered in fewer time points. In fact in the cortex shorter sounds elicit larger responses than longer sounds (Beukes et al., 2009; Wang et al., 2016). Overall, this is consistent with the pattern I observe, and the differences might be only due to the physical properties of the used stimuli. This suggests that in $\mathrm{MCT} 1^{+/}$amplitude impairments are the leading effect, something that might be correlated with a change in the spiking threshold of individual axons.

\section{Adaptation and temporal reliability}

Both mutant types showed a deficit in rate coding that was independent from the response amplitude. This deficit was reflected in the normal response to the initial clicks in a sequence and progressive adaptation as stimulation continued, which could be translated to decreased rate coding. The Hif1 $\alpha^{\text {fl/fil }}$ PLP-creERT2 ${ }^{+}$mice differed from the $\mathrm{MBP}^{\text {shi }}$ in that they showed an increase in spike desynchronization in the middle clicks only, while the final clicks were no different from control animals (Figure 43). Interestingly, when translating these responses to the analysis of spike synchrony, the Hif $1 \alpha^{f / f f l} ; \mathrm{PLP}$-creERT2 ${ }^{+}$mice showed very strong spike desynchronization and it would appear that these animals have a stronger desynchronization 
than the $\mathrm{MBP}^{\text {shi }}$. Nevertheless, this is not the case and this might have to do with a possible bias from the way the analysis was performed. While $\mathrm{MBP}^{\text {shi }}$ mice were unresponsive to the last clicks, Hif $1 \alpha^{f l / f l} ;$ PLP-creERT2 ${ }^{+}$mice showed a strong decrease in response amplitude to the middle clicks. The stronger desynchronization effect seen in the Hif1 $\alpha^{f / f f} ;$ PLP-creERT2 ${ }^{+}$ mice might come from the fact that for the synchrony analysis, a sliding window covering 4 clicks was used, the 6 windows measured comprised from click 2-5, 3-6, 4-7, 5-8, 6-9, and 710. As a result, the middle clicks were overrepresented in the data due to this technique. It is not clear, however, what is the mechanism of this mid-train response decay.

Neuronal firing requires a lot of energy. My data indicates that trophic support from oligodendrocytes is important to maintain normal levels of firing probability. Reports indicate that GABAergic cells might require much more energy than other cell types, due to their fastspiking activity (Kann, 2016; Kann et al., 2014; McCasland and Hibbard, 1997). A particular subset of GABAergic interneurons, the $\mathrm{PV}^{+}$interneurons, are central players in cortical processing, especially important for the maintenance of oscillatory activity (Kann et al., 2014). This oscillations, especially the ones happening at high frequency ranges, are essential for cognitive tasks (Buzsáki and Wang, 2012) and use high amount of energy (Huchzermeyer et al., 2013; Kann, 2016; Kann et al., 2011, 2014). Interestingly, it is these PV+ interneurons that are preferentially myelinated in cortical areas (Micheva et al., 2016; Stedehouder et al., 2017) and also appear to play a role in cortical gap-detection (Keller et al., 2018; Weible et al., 2014a). It appears that this special high-spiking subpopulation of neurons in the cortex would be singularly susceptible to a deficit in the metabolic support from glial cells. It would be interesting to study the role of myelin and metabolic support in this subpopulation of interneurons to further understand the importance of glial-trophic support in circuit function.

Temporal acuity was measured using gap-detection and it was found affected in the

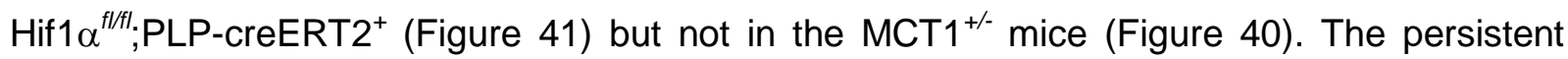
effect observed was a strong reduction in spiking probability. Gap-detection does not require the ability to fire at high rates, but the temporally precise response recovery. The reduction in temporal acuity of these animals was surprisingly similar to what I observed with the MBP ${ }^{s h i}$. These results suggest that axons that are enwrapped by non-metabolically stable oligodendrocytes have a reduction in the spiking probability when two stimuli are presented close in time. In Chapter 1, I proposed that the gap-detection deficit observed in the MBP ${ }^{\text {shi }}$ mice was the result of increased refractoriness due to changes in ion channel expression and 
location. Nevertheless, this might not hold true for the Hif $1 \alpha^{\text {fl/fl }}$;PLP-creERT2 ${ }^{+}$, since I do not expect a striking change in the distribution or expression of axonal ion channels. The $\mathrm{Na}^{+} / \mathrm{K}^{+}$ ATPase pump is involved in refractoriness and requires a lot of energy (Alizadeh et al., 2015), and energetic stores might be affected in the mouse models here assessed. Changes in the activity of the $\mathrm{Na}^{+} / \mathrm{K}^{+}$ATPase might also be causing some of the effects observed here. Testing whether these mice show differences on the nodal channels, the AIS, or the activity of the $\mathrm{Na}^{+} / \mathrm{K}^{+}$ATPase would provide more information regarding the mechanisms of this change in gap-detection.

\section{Is anesthesia playing a role?}

The use of anesthesia in assessing neuronal responses in the cortex can result in dampening of neuronal responses (Goldstein Jr. et al., 1959), and can change the availability of metabolites in the brain (Mächler et al., 2016), which could potentially affect spiking activity. The specifics of how the anesthesia that I used affects availability of lactate in the brain are not known and were not studied further. Nevertheless, anesthetic conditions were maintained similarly for both control and mutant animals, suggesting that differences observed arise from an axonal defect due to loss of metabolic glial support. The ultimate goal of this study was to address how changes in glial-metabolism impact the axons. Whether this was a long-term effect that arose with the mutation, or a short-term effect being reflected in the immediate response of the axons upon stimulation is not known, and it will be probably very difficult to dissociate in these experimental conditions. This issue could be addressed by performing in vivo awake recordings in these animals.

To summarize, the metabolic impairments of myelinating glia or the disruption of axoglial metabolic support impair axonal function in processes that go beyond a simple loss of conduction velocity. Some of the mechanisms that underlie the loss of temporal processing, might be shared in patients with metabolic pathologies. For instance, it has been reported that diabetic patients show poorer discrimination of gaps in sound (Mishra et al., 2016). De/dysmyelination, which would be minimal in the mouse models used in this chapter, is not playing an important role in the neuronal effects I observed. Mainly, affecting the metabolism of myelinating glia has a strong impact on auditory temporal processing, most probably to an overall metabolic instability of the wrapped axons. Here I showed that even though it is complicated to dissect the partially independent roles that myelin plays in the stability of axons, both strong dysmyelination and a glial-metabolic dysfunction elicit similar effects in 
axons. Part of the deficits observed from dysmyelination (Chapter 1) might result due to deficits in metabolic support from the oligodendrocytes stemming from a reduction in axonal interactions. This proves that oligodendrocyte trophic support is also important for the maintenance of higher brain functions and goes beyond.

\subsection{Conclusions}

Oligodendrocytes are key players in processes that require the adjustment of conduction velocity along specific circuits in the brain. Even though their ancient dominant role was the speeding up of action potential propagation, the less obvious observations regarding myelin function are related to an important role of trophic support to the axons. Additionally, the proper interaction between oligodendrocytes and axons, and the placement of internodes, is playing a role in the stability and the architecture of axonal excitable domains, which is one determinant of conduction speed. Increasing evidence is accumulating that shows that the oligodendrocytes have many faces in brain function (Figure 44).

Interestingly, developed myelinating oligodendrocytes that have successfully wrapped axons reduce myelin content when confronted to a metabolic restriction, probably as a way of obtaining an extra energy source. Axons that have to cope with metabolically impaired oligodendrocytes or with a reduction on the trophic support, show abnormalities similar to the ones that undergo dysmyelination. This suggests that the detachment of myelin sheaths from axons per se is not only causing a reduction in action potential propagation, or conduction blocks, but it is also impairing processes that need the close contact and communication between axons and glial cells. This study, shows for the first time, the functional correlation of these two processes using the auditory system as a model to study sensory processing. The defects found not only impair basic neuronal capabilities, but disrupt cortical features that are needed for higher-order processing. 


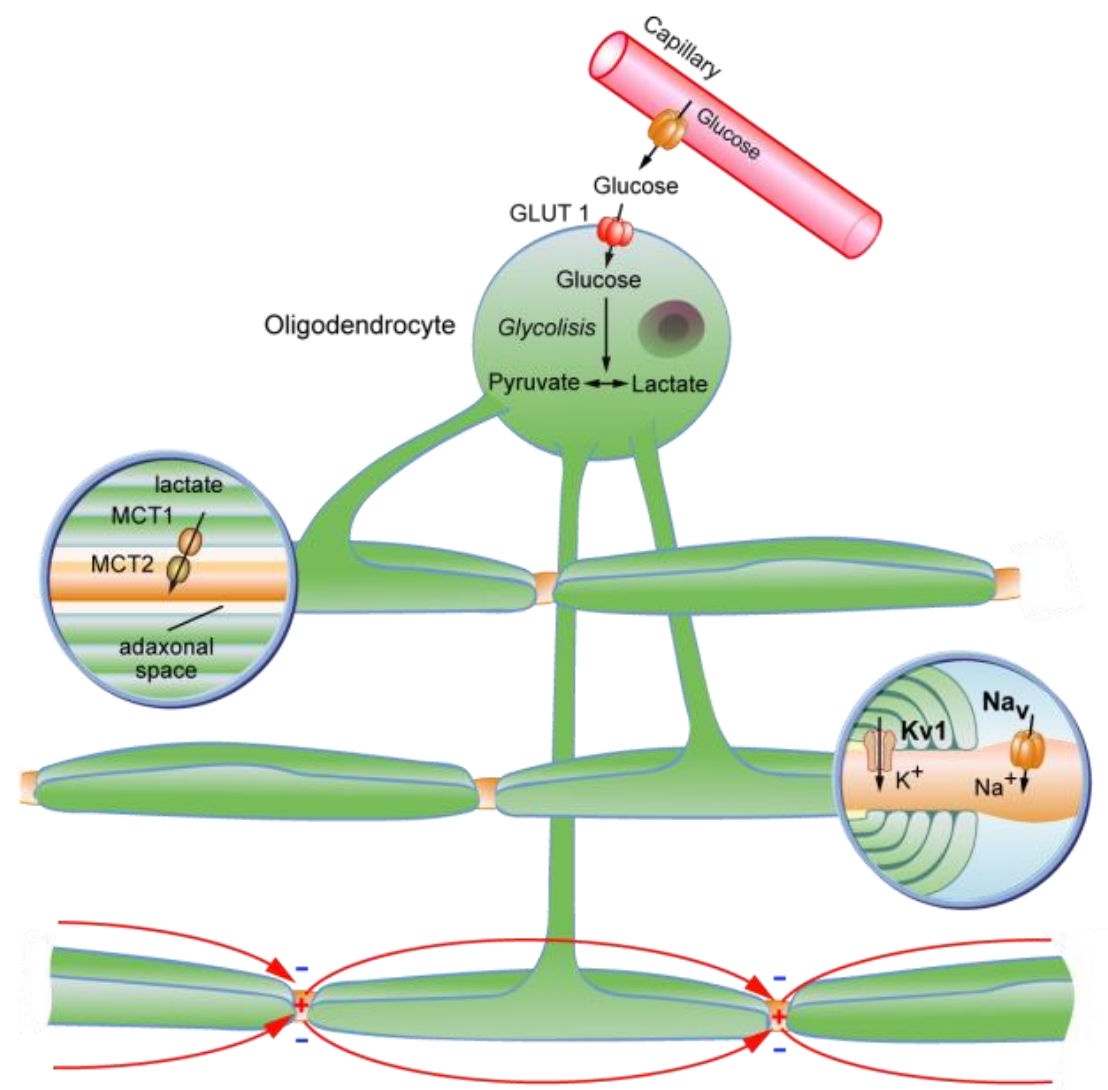

Figure 44. The diverse roles of oligodendrocytes for the maintenance of axonal stability.

Glycolytic oligodendrocytes can support axons via the transport of glycolytic end products, such as lactate or pyruvate (upper axon). The maintenance of axonalexcitable domains and the clustering of ion channels are essential for saltatory conduction (middle and lower axons).

Image credit: Jan Ficner. 


\section{Chapter 3}

\section{Myelin and the establishment of behavioral laterality}

\subsection{Introduction}

In this final chapter, my aim is to relate the functionality of the largest white matter tract in the brain (the corpus callosum), to a behavioral function that it is believed relies on its activity: handedness. In this specific case, I focus on the study of paw-preference in mice with different degrees of dysmyelination as a paradigm to study the functionality of white matter in behaving animals.

\subsubsection{Lateralization of brain function}

The lateralization of brain function is an evolutionary adaptation that contributes to the fitness of an organism (Vallortigara et al., 1999). It refers to the fact that certain tasks tend to generate higher activation, or require specific brain areas located in one of the two brain hemispheres. Hemispheric lateralization allows the two hemispheres to perform different computations and probably, save reduce the cost of information processing. Increasing amount of evidence suggests that lateralized functions are also encountered in most vertebrates (Rogers, 2009; Rogers and Andrew, 2002; Vallortigara et al., 1999). In humans, functional asymmetries have been mainly associated with language processing and limb dominance. In the case of limb dominance, by definition, the dominant limb is the one used to manipulate or mobilize objects (e.g. food), while the non-dominant limb usually lends postural 
support (Gabbard and Hart, 1996) (see Figure 45). Despite the existence of evidence for brain asymmetries since the 1880' (Berker, 1986), many controversies surround the topic of brain lateralization; from the mechanisms that are involved in its establishment, to the real function that serves.

One of the main players for the establishment and maintenance of lateralized functions is proposed to be the corpus callosum (CC) (Cassells et al., 1990; van der Knaap and van der Ham, 2011). The CC is the largest white matter tract in the brain, and it is an important connection between both hemispheres (Gazzaniga, 2000). Nevertheless, the exact mechanisms of how the CC modulates interhemispheric information transfer are still under debate. Two main theories have been proposed (reviewed in (van der Knaap and van der Ham, 2011): 1) the inhibitory theory, which suggests that an increase in lateralization is seen as an enhanced inhibition of the contralateral hemisphere, which will allow the task-related 'dominant' hemisphere to control specific type of processing. 2) The excitatory theory suggests that there is a complex intercommunication and information integration between both hemispheres that results in the activation of the less active hemisphere. To date, it is not yet clear how these interactions take place and the evidence to favor one or the other theory appears divided (reviewed in (van der Knaap and van der Ham, 2011). It is important to highlight that when talking about hemispheric dominance, it does not mean that the other hemisphere becomes 'dormant' or inactive, but that the more active hemisphere plays a greater role in a specific task.
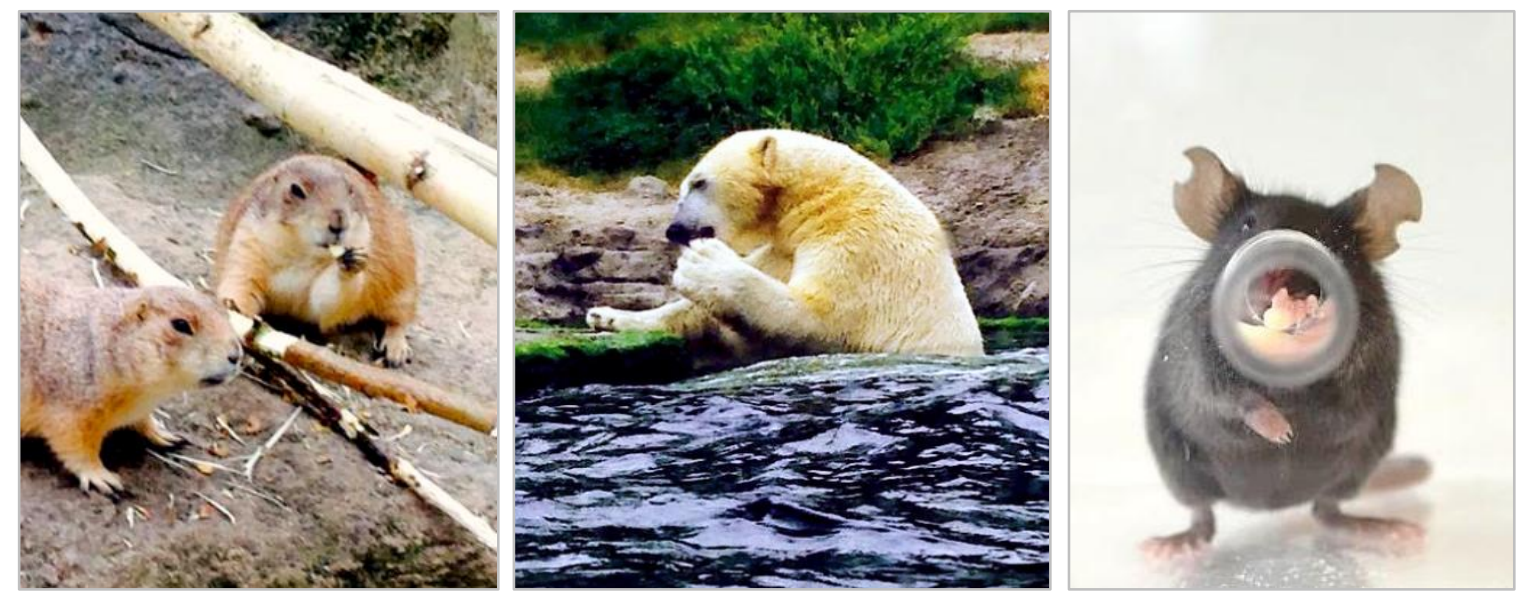

Figure 45. Patterns of behavioral laterality can be found in most vertebrates.

Examples of different vertebrate species showing behavioral lateralization during feeding. Leftmost picture shows a prairie dog using the dominant (left) paw for eating, while the non-dominant one is helping it equilibrate. Same observation observed in a polar bear feeding in the water (middle). Our animal model (right) showing a similar behavior when tested with the paw-preference task. 
In this chapter, I focused in only one type of lateralized function in mice: paw preference. Because of that, from now on, any references made to lateralized functions must be taken only in the context of paw dominance or hand laterality, which I will commonly term 'behavioral laterality'. When referring to laterality, only two aspects will be taken into account. One is the direction of laterality, which refers to the side preference (right or left paw usage); the other one is the strength, which is the value of consistency of the asymmetry (Preslar et al., 2014). Both measurements show impairments in certain conditions that have been associated with dysfunctions in the CC or with myelin abnormalities.

Research of certain pathological states affecting the CC (e.g. agenesis of the CC), or chirurgical procedures (i.e. callosotomy), both in humans and animal models, has provided a lot of information regarding the function of the CC, and how the hemispheres communicate. It has been reported that congenital absence of the CC reduces the strength of paw lateralization in mice (Gruber et al., 1991), or can generate a leftward bias (Manhães et al., 2005). Newborn or adult rodents that had a callosotomy (chirugical separation of the CC) also present a leftward bias in paw preference (Manhães et al., 2003; Miu et al., 2006). In these studies, it is suggested that changes in the transfer of information between hemispheres accounts for the differences in paw preference strength or directionality. In humans and animal models, it has been demonstrated that autism and schizophrenia, two diseases that can be associated with myelination deficits (Carmody and Lewis, 2010; Fingher et al., 2017; Haroutunian et al., 2014; Miller et al., 2013; Pacey et al., 2013; Pajevic et al., 2014; Satz and Green, 1999; Stedehouder and Kushner, 2017; Takahashi et al., 2011; Vidal et al., 2006; Wei et al., 2016; Xu and Li, 2011), are also associated with higher prevalence of left-handedness (Cornish and McManus, 1996; Dollfus et al., 2012; Gillberg, 1983; Preslar et al., 2014; Vidal et al., 2006) or reduced manual preference (Dollfus et al., 2012). In addition, some marsupials, which lack a CC (Karlen and Krubitzer, 2007) have been recently reported to have a strong leftward forelimb bias (Giljov et al., 2015).

The information here presented, suggests that the CC is playing an essential role in the interhemispheric communication and is essential for adequate information processing in the brain. Being the largest white matter tract, it could be an area especially susceptible to impaired function when myelin is disrupted. The study of the CC functionality in relation to myelin loss might shed light on circuit-specific interactions and their modulation by white matter in the brain. 


\subsection{Results}

The aim of this section was to understand how myelin abnormalities can affect the cognitive function of an organism. In this case, by testing behavioral lateralization in mice with different dysmyelination levels, I aim to find a behavioral correlate of a task that depends on the functionality of the largest white matter tract in the brain: the corpus callosum.

I started testing behavioral laterality in $\mathrm{MBP}^{\text {shi }}$ mice using the paw-preference test. Briefly (as mentioned in Materials and Methods section II.X.I), mice are put in a testing-box, which has a feeding tube in the frontal wall. Mice are food restricted and motivated to reach with the paws for pellets of food that are placed inside the feeding tube. Only one paw can be used to retrieve the pellet. The amount of reaches with the right or left paws is recorded and analyzed to obtain a measurement of the strength (consistency of paw usage) and direction (right or left paw usage) of behavioral laterality. $\mathrm{MBP}^{\text {shi }}$ mice show a strong shivering phenotype, which is mainly seen in the hind limbs and during active movement. But when the $\mathrm{MBP}^{\text {shi }}$ mice were engaged in the task, the shivering phenotype in the short time that comprised the paw reach was minimal, which allowed them to perform the task skillfully. The first issue that I addressed was to confirm if $\mathrm{MPB}^{\text {shi }}$ mice were as efficient as control animals in retrieving the pellets and performing the task. For this, two measurements were used: one was the total amount of paw entries in the testing session, and the other one was the retrieval efficiency. The total amount of paw entries during a testing session was not different between groups $(p=0.35)$ (Figure 46-A). Nevertheless, when measuring the amount of entries that were required to retrieve a pellet $\mathrm{MBP}^{\text {shi }}$ mice to needed in average, less than 3 attempts instead of 2.5 as in control animals $(p=0.046)$ (Figure 46-B). Although this difference was significant, the overall effect was minimal and did not have a strong impact on performance, since the total amount of paw entries was not different from control animals. Thus, mice were tested in 2 consecutive days. The first day consisted of a 'training' session which allowed the animals to familiarize with the environment and the test. One day after, the 'testing' session was performed. This allowed me to test for effects of the training in the learning of this task that could potentially impact the measurement of laterality. In this case, I made a comparison between the preferred paw entries on the day of training and the day of testing. I found that animals showed similar lateralization scores between training and testing (Figure 46-C). A paired t-test confirmed no significant differences for control $(p=0.24)$ and mutant $(p=0.44)$ animals. 
A

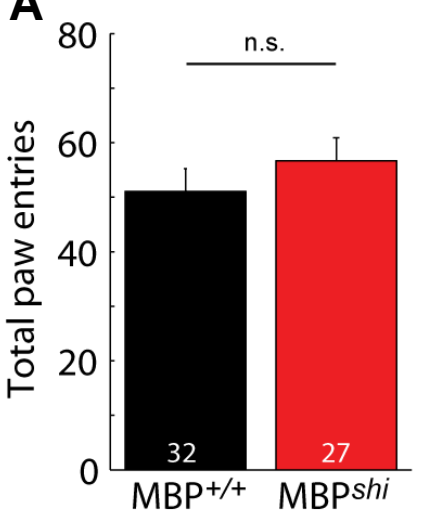

B

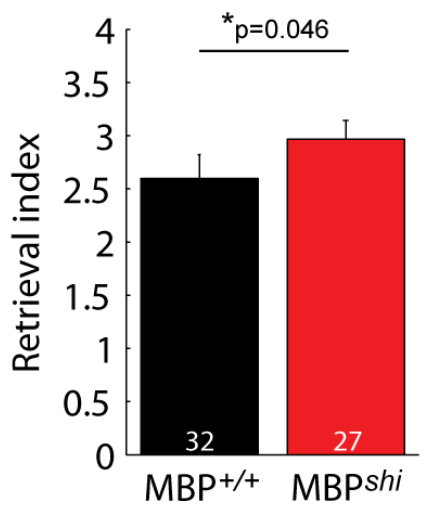

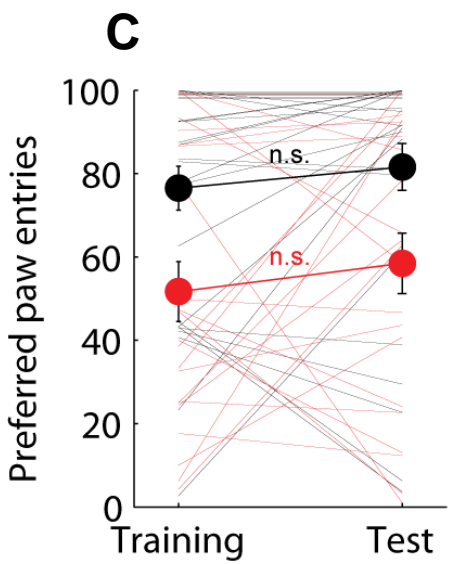

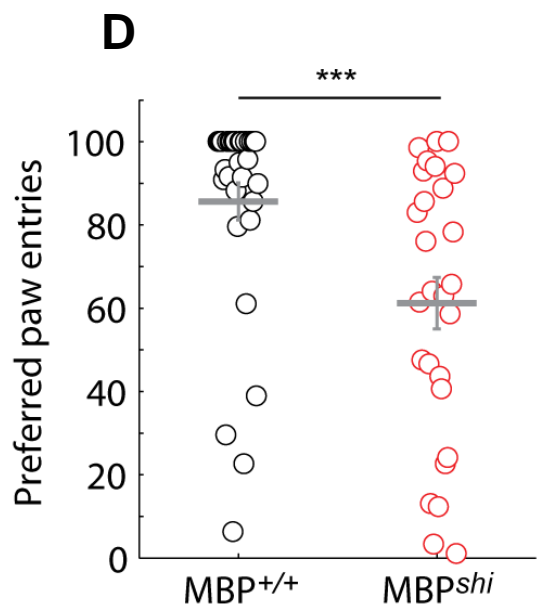

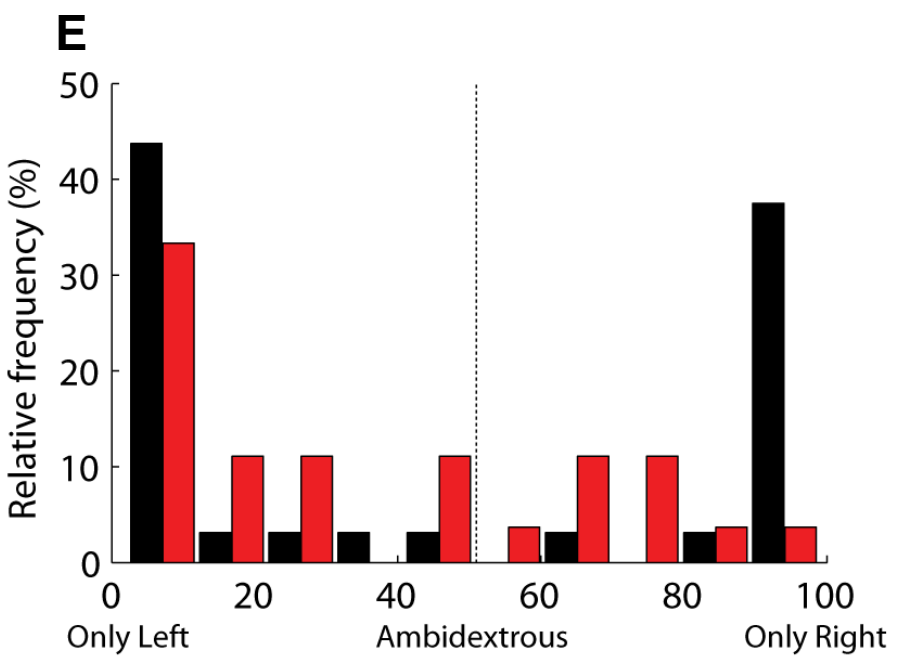

Figure 46. Behavioral laterality is impaired in $\mathrm{MBP}^{\text {shl }}$ mice.

\%Right paw entries

A) The quantification of total paw entries during the test session was done, revealing no significant differences between control (black) and mutant (red) animals ( $p=0.35$ ), suggesting both groups had similar performance. A more direct measure of performance was obtained by measuring the amount of reaches necessary to efficiently retrieve one food pellet. $\quad$ B) Shows the quantification of the retrieval index, where MBP ${ }^{\text {shi }}$ mice show a mild but significant increase $(p=0.046)$, meaning that $\mathrm{MBP}^{\text {shi }}$ mice had to do in average, less than one more paw entry, compared to control animals, to efficiently retrieve one pellet. The comparison between the consistency of lateralization between the training and the test session was done. $\ln \mathrm{C}$ ) we can observe that both groups showed very similar strength of lateralization scores between sessions, without significant differences within each group (paired t-test, $\mathrm{p}=0.24$ for controls and $\mathrm{p}=0.44$ for mutants). $\mathrm{n}=29$ control (black) and $\mathrm{n}=22$ mutant (red) mice. Solid circles show the mean per group and condition. D) Shows the quantification of laterality strength during the testing day. MBP ${ }^{\text {shi }}$ mice (red) have significantly lower laterality scores compared to controls (black) $(\mathrm{p}=0.0003)$. E) Shows the population distribution of laterality direction. While control animals (black) usually have a ' $U$ ' shaped distribution, with the majority of the animals being either right-pawed (\%right paw entries $=100$ ) or left-pawed (\%right paw entries $=0$ ), and a small population of ambidextrous animals (\% right paw entries between 20 to 80 ). MBPshi mice have a skewed distribution, having similar amount of left-pawed animals, while a strong reduction in the population of right-pawed animals, and an increase in the ambidextrous is seen. In all cases, error bars represent the S.E.M. 
The quantification of the strength of laterality was done for the data obtained in the test session. $\mathrm{MBP}^{\text {shi }}$ mice show a strong reduction in the strength of the lateralization (percentage of preferred paw entries) compared to control animals ( $p=0.0003$ ) (Figure 46-D). This value reflects the consistency of paw usage independently of the preferred direction. This means that $\mathrm{MBP}^{\text {shi }}$ mice tend to alternate more between paws during the pellet retrieval task, while control animals tend to use uniquely the preferred paw. In addition to a change in the strength of laterality, I observed a difference in the directionality of lateralization, reflected in a different population distribution between control and mutant animals. Here it is important to mention that different mouse strains have been reported to have different laterality scores (Biddle et al., 1993; Takeda and Endo, 1993). In the case of mice under a C57BL/6 background, it is known that they present a ' $U$ ' shaped laterality distribution, with high percentage of animals being either left-pawed or right-pawed, and a very small percentage being ambidextrous (Signore et al., 1991). While control animals showed the typical ' $U$ ' shape distribution with the majority of them being either right or left-pawed, and a minority ambidextrous, the directionality distribution in $\mathrm{MBP}^{\text {shi }}$ mice is skewed $\left(\mathrm{X}^{2}(9)=269.17\right.$, $\mathrm{p}<0.0001)$. I observed that the amount of left-pawed animals was not changed, but there were few $\mathrm{MBP}^{\text {shi }}$ mice that were strongly lateralized to the right (Figure 46-E). In addition, an increase on the amount of ambidextrous mice was observed.

These results suggest that $\mathrm{MBP}^{\text {shi }}$ mice had a strong impairment in the consistency of paw usage, but also, a change in the population distribution of direction preference. If these effects are related to a reduction of CC functionality, it would mean that certain specificities of the establishment of behavioral lateralization might be regulated differently between hemispheres, since I observed a preferential impairment in the right-pawed population.

It is important to remember that the $\mathrm{MBP}^{\text {shi }}$ mice have an overall dysmyelination phenotype in the CNS, and not one related uniquely to the CC. I was interested to understand more specifically the relationship of CC dysmyelination and the effects on behavioral laterality. For this reason, I tested the $\mathrm{MBP}^{e m x}$ mice, which have forebrain-specific dysmyelination. Similar to what was observed in the $\mathrm{MBP}^{\text {neo }}$ mice, no differences were found regarding the strength of lateralization $(p=0.38)$ (Figure 47-A). For the direction of lateralization, no statistical analysis was performed due to reduced animal number in the control group. Nonetheless, both groups showed a similar ' $U$ ' shape distribution (Figure 47-B) of behavioral lateralization. Posterior careful characterization of the myelination profiles of the 
$\mathrm{CC}$ revealed that the ventral part of the $\mathrm{CC}$ in $\mathrm{MBP}^{\mathrm{emx}}$ mice is normally myelinated. This could be the reason why no effects were seen in these animals. Nonetheless, I can conclude that the lack of myelin in intra-cortical circuits was not relevant for the establishment of behavioral laterality. A similar effect is observed in $\mathrm{MBP}^{\text {neo }}$ mice with partial dysmyelination (data not shown).
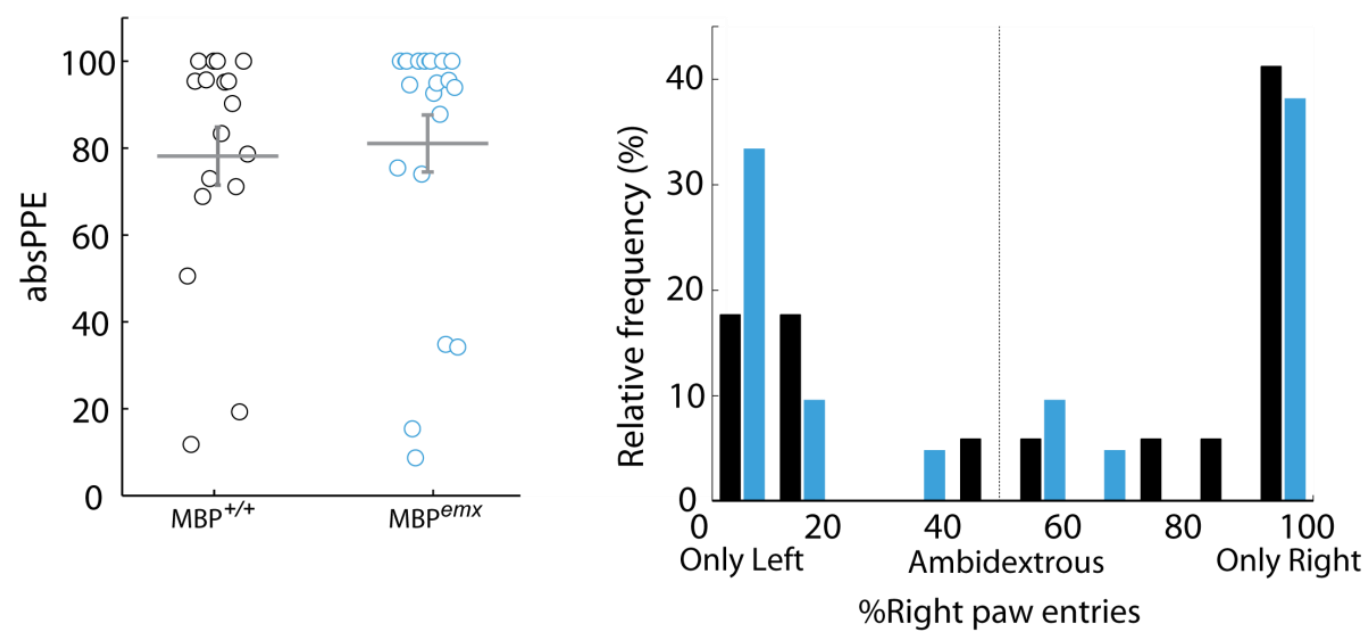

Figure 47. Behavioral laterality is not impaired in $\mathrm{MBP}^{\mathrm{emx}}$ mice.

A) The strength of lateralization is shown for control (black) and MBP ${ }^{e m x}$ (blue) animals. We can observe that both groups showed very similar strength of lateralization scores $(p=0.38)$. Thick gray bars depict the mean per group and error bars represent the S.E.M. B) Population distribution of laterality direction. Both groups show the typical ' $U$ ' shaped distribution. A tendency towards a larger population of left-pawed animals is seen in the mutant group. $n=17$ control and $n=21$ mutant mice.

So far I have observed that a total reduction in myelin during development impairs behavioral laterality. This is maybe not surprising, since impairing the synchrony of interhemispheric information transfer during development is expected to cause defects in the establishment of certain circuits. My next question was whether a loss of myelin during adulthood, once the circuit has been established and the peak of myelination has passed, could affect this behavior. For this reason, I used an inducible MPB ${ }^{f / / f} ; \mathrm{PLP}^{-c r e E R T 2}{ }^{+}$mouse model, which upon tamoxifen injection at 8 weeks of age, starts demyelinating, presenting a peak of dysmyelination between 6-8 months after tamoxifen induction. The peak of dysmyelination correlates with a slight tremor that the animals present at that age, and upon remyelination, the tremor disappears ( $\sim$ year after tamoxifen induction). These mice were tested during the peak of demyelination. I found no differences between MPB ${ }^{f / f l}$;PLPcreERT2 $^{\text {wt }}$ and $\mathrm{MPB}^{\mathrm{fl} / f l} ; \mathrm{PLP}_{\mathrm{C}} \mathrm{creERT2}{ }^{+}$animals regarding the amount of paw entries during 
the test session $(p=0.28)$. Additionally, no changes in strength $(p=0.96)$ were observed (Figure 48-A). Regarding the directionality of lateralization, no statistical analysis was performed due to the low animal number (below 20). Nevertheless, it seems that there is a slight increase in the population of left handed animals (Figure 48-B).

A

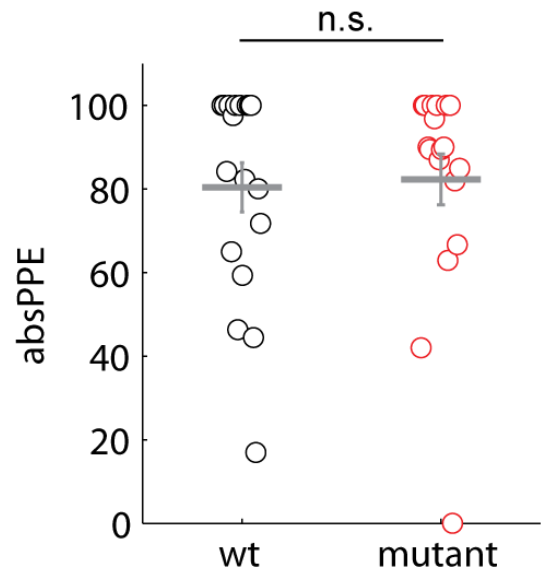

B

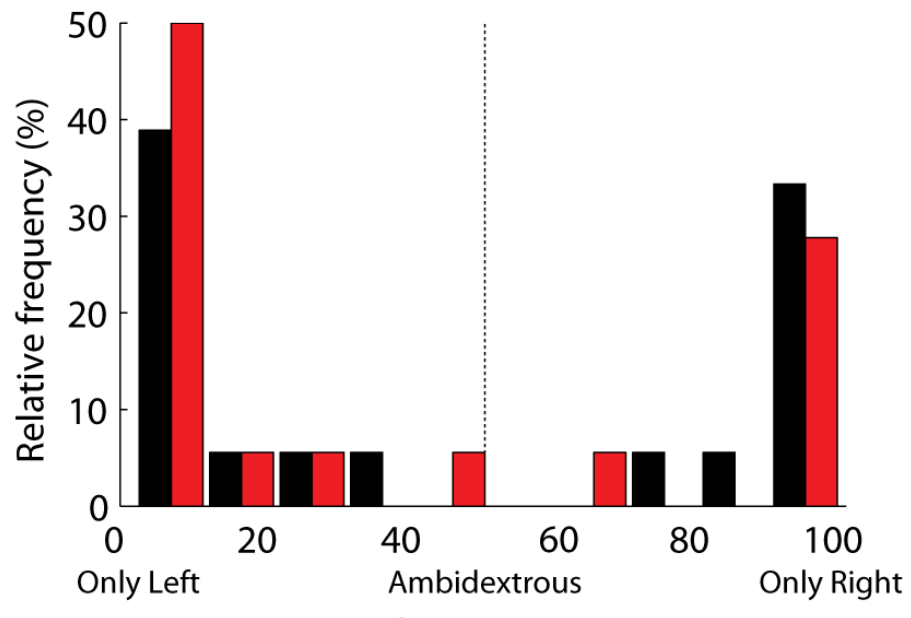

\%Right paw entries

Figure 48. Behavioral laterality is not impaired in $\mathrm{MBP}^{t / \pi} ; \mathrm{PLP}^{-c r e E R T 2^{+}}$mice.

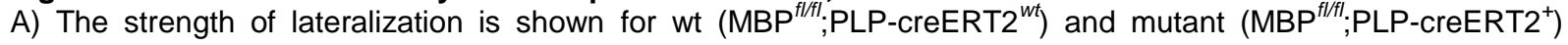
animals. We can observe that both groups showed very similar strength of lateralization scores $(p=0.96)$. Thick gray bars depict the mean per group and error bars represent the S.E.M. B) Population distribution of laterality direction. Both groups show the typical ' $U$ ' shaped distribution. A tendency towards a larger population of leftpawed animals is seen in the mutant group (red). $n=18$ control (black) and $n=18$ mutant (red) mice

Taking all these data together, a strong impairment in myelin formation reduces the consistency of paw usage, and creates a bias in the population distribution of directionality of behavioral lateralization. The latter might be due to the fact that right-pawed animals seem to become ambidextrous, while the population of left-handed animals remains unaffected. 


\subsection{Discussion}

One of the aims of this project was to study of the role that myelin plays in network functionality. The aim of this chapter was to understand the correlation between corpus callosum functionality and myelin disturbance in the behaving animals. I studied pawpreference, as a measure of laterality, in mice with different degrees of dysmyelination in the brain, to understand how myelin is regulating the establishment and maintenance of one possible circuit responsible for this behavior. The paw-preference test has been proven as a reliable test for the analysis of laterality in rodents (Barnéoud and Van der Loos, 1993; Collins, 1968; Collins et al., 1993; Manhães et al., 2003; McManus, 1992; Ribeiro et al., 2014; Ribeiro-Carvalho et al., 2010; Signore et al., 1991; Takeda and Endo, 1993; Waters and Denenberg, 1994). Even though it was initially developed to assess the heritability of handedness in mice, nowadays variations of this test are widely used to understand circuits implicated in reaching movements (Azim et al., 2014; Ellens et al., 2016; Guo et al., 2015; Hira et al., 2015; Lai et al., 2015; Wang et al., 2017).

In these experiments, high levels of laterality strength were seen in control animals since the training session, and no differences were observed between training and testing sessions. This suggests that at the age these animals were tested, behavioral laterality was already an established feature. When measuring the strength and directionality of lateralization, I found that MPB ${ }^{\text {shi }}$ mice have significantly lower levels of lateralization strength (Figure 46-D), which means these animals tend to use both paws for the retrieval of a food pellet and are not consistent in the usage of one preferred paw as seen with control animals. In addition, the population distribution of directionality preference in $\mathrm{MBP}^{\text {shi }}$ mice shows a skewed distribution. I was able to replicate this typical ' $U$ ' shaped distribution in control mice (Figure 46-E). Nevertheless, the population of right handed $\mathrm{MBP}^{\text {shi }}$ is almost inexistent and an increase in the amount of ambidextrous animals is seen (Figure 46-D and E). When measuring laterality scores in mice with an only-forebrain dysmyelination (MBP ${ }^{e m x}$ ) (Figure 47), I observed no changes in this behavior, and a similar effect was observed in $\mathrm{MBP}^{\text {neo }}$ mice with partial dysmyelination (data not shown). The results of the $\mathrm{MBP}^{\mathrm{em} x}$ mice were unexpected, since the mutation should be affecting the whole CC. Closer EM examination of the more ventral areas of the corpus callosum revealed that there is a gradient of dysmyelination from dorsal to ventral areas, showing that the ventral part of the $\mathrm{CC}$ is normally myelinated (data not shown). These results could mean on one side, that normal myelination in the ventral corpus callosum is enough to maintain an adequate 
interhemispheric information transfer, and to develop normal levels of strength and directionality of lateralization. On the other hand, it could mean that inter-cortical interactions (e.g. within different motor areas) which play a role in the actual movement planning and execution (Guo et al., 2015), are less likely involved in the establishment of behavioral laterality. Finally, it seems that affecting the circuitry during adulthood by means of demyelination does not impact paw-preference (Figure 48).

Changes in gene expression in the spinal cord have been implicated in the establishment of handedness (Ocklenburg et al., 2017). My results could likewise, be influenced by changes that go beyond a direct loss of CC function, but only to an overall dysmyelination phenotype. Testing mice with CC-specific demyelination may help understanding the specific role of the $\mathrm{CC}$ in behavioral lateralization.

Observing a reduction in the strength of lateralization in the $\mathrm{MBP}^{\text {shi }}$ mice was unexpected only to a certain extent since there is a severe disruption of the circuitry due to complete CNS dysmyelination. Response delays and desynchronization between the information that is being transferred from one to the other hemisphere might account for the reduced strength of laterality in $\mathrm{MBP}^{\text {shi }}$ mice. These differences might be conferred by the types of fibers and the callosal microstructure. It has been suggested that individual variabilities in the CC account for the differences of timing of signals between hemispheres (Friedrich et al., 2017). Interestingly, the observation that the leftward-pawed population does not seem to be affected as much as the right-pawed one is striking. This bias has constantly been reported in the literature for animals with callosal agenesis, callosotomy or for the human population with autism and schizophrenia (see section 3.1.1). A leftward bias is the common factor in all these states that are also associated with a callosal dysfunction even though the distribution of pawedness/handedness at the population level in rodents and humans is very different. The fact that I observed a similar effect with the $\mathrm{MBP}^{\text {shi }}$ mice could suggest that there is a 'default' functionality of the circuitry in charge of behavioral lateralization in mice; upon development, with myelination, the circuitry becomes less asymmetrical. At this point, probably there is a critical window where the experience and paw usage of the animal allows the shaping and establishment of behavioral laterality. It is possible that there is an asymmetry of the fibers that travel along the CC or in specific CC regions involved in behavioral lateralization, as it has been shown in the differences of fiber diameter along the CC in humans (Aboitiz and Montiel, 2003). Additionally it has been suggested that individual 
variations in myelin genes could affect the $\mathrm{CC}$ and modify interhemispheric integration (Ocklenburg et al., 2016a, 2016b, 2018). Depending on the conduction velocity of these fibers, an architectural reason could be the cause of the asymmetries I see in the MBP $\mathrm{M}^{\text {shi }}$ mice.

Overall, these data suggest that the transfer of information along pathways that are mediating laterality can be disrupted with dysmyelination, which leads to abnormalities related to behavioral lateralization. Partial dysmyelination or demyelination during adulthood does not generate strong changes in the circuity that impact the behavioral output. This pattern suggests again that it is not conduction velocity that modulates lateralization, for this is not affected in partial dysmyelination or adulthood myelin disturbances. This represents another yet unknown factor by which myelination shapes brain circuits. 


\section{Summary and Conclusions}

Myelination is a beautifully complex process that requires the orchestration of cell-to-cell communication, the development of progenitor cells, the recognition of axonal targets, wrapping, compaction, and stabilization, together with adequate mechanisms to ensure the stability of the already formed myelin sheaths. Myelin is crucial for efficient information processing in the brain, whose function depends on the regulation of synchronous inputs within different circuits.

In this study, the auditory system, which requires a high temporal precision, was the preferred model to study the how sensory processing, perception, and cognition are affected by myelin deficits. I used in vivo electrophysiology techniques and behavioral paradigms to test the auditory function of different mutant mice within the spectrum of oligodendrocyte abnormalities. By measuring sound-evoked spiking activity of neurons from the cortex, I observed a reduction in temporal processing. My observations suggest that mice with complete or partial dysmyelination along the auditory system show abnormalities both in temporal acuity (gap-detection) and temporal reliability (rate coding). Parallel behavioral tests showed that perceptual gap-detection was also impaired in mice with partial dysmyelination, suggesting a robust neuronal correlate of this behavior. None of the deficits could be related to a peripheral impairment in sound processing, or a general auditory problem, and were mainly constrained to central temporal sound processing. These temporal deficits cannot be explained uniquely by a reduction in conduction velocity and might be caused by underlying mechanisms secondary to the loss of myelin, such as changes in the expression and localization of channels in axonal excitable domains, or changes in the glial metabolic support. Interestingly, the key effects seen upon dysmyelination, namely the temporal deficits, were also replicated, to a similar extent, in mice with an axo-glial dysfunction that impacts the metabolic stability of axons. These results suggest that for adequate firing and coding of information, axons along the auditory system require not only the presence of healthy and compact myelin but also trophic support and metabolic stability from the oligodendrocytes.

Furthermore, I tested the role of myelin in the functionality of circuits related with behavioral laterality in mice. The results suggest that the formation of compact myelin during development might be playing a role in the establishment of behavioral laterality. A partial reduction in myelin compaction or a demyelination during adulthood would not impact this behavior. 
Even though several sensory and cognitive disruptions have been reported in patients with diseases related to myelin, the complexity of such pathological states in humans has made it difficult to interpret the role of myelin in these abnormalities. Here I also showed that the use of different oligodendrocyte-impaired animal models and specific auditory and behavioral tests, proved to be efficient in dissecting the roles that myelin disturbances can be causing. The careful study of specific higher-order processes might help better understand certain myelin pathologies.

To wrap up, this study shows that myelin plays a role in the reliability of sensory and cognitive processing, that this role goes beyond merely regulating conduction velocity, and is essential for higher-order processing such as speech understanding. This reinforces the idea that the role of oligodendrocytes function in sensory and cognitive processes proves to be more complex than what was thought in the past. 


\section{References}

Aboitiz, F., and Montiel, J. (2003). One hundred million years of interhemispheric communication: the history of the corpus callosum. Braz J Med Biol Res 36, 409-420.

Alizadeh, A., Dyck, S.M., and Karimi-Abdolrezaee, S. (2015). Myelin damage and repair in pathologic CNS: challenges and prospects. Front Mol Neurosci 8, 35.

Allen, P.D., Schmuck, N., Ison, J.R., and Walton, J.P. (2008). Kv1.1 channel subunits are not necessary for high temporal acuity in behavioral and electrophysiological gap detection. Hear Res 246, $52-58$.

Alviña, K., and Khodakhah, K. (2010). The therapeutic mode of action of 4-aminopyridine in cerebellar ataxia. J Neurosci 30, 7258-7268.

Anderson, L.A., and Linden, J.F. (2016). Mind the gap: two dissociable mechanisms of temporal processing in the auditory system. J Neurosci 36, 1977-1995.

Aragonés, J., Fraisl, P., Baes, M., and Carmeliet, P. (2009). Oxygen sensors at the crossroad of metabolism. Cell Metab 9, 11-22.

Arancibia-Cárcamo, I.L., Ford, M.C., Cossell, L., Ishida, K., Tohyama, K., and Attwell, D. (2017). Node of Ranvier length as a potential regulator of myelinated axon conduction speed. Elife 6 .

Arroyo, E.J., Xu, T., Poliak, S., Watson, M., Peles, E., and Scherer, S.S. (2001). Internodal specializations of myelinated axons in the central nervous system. Cell Tissue Res 305, 53-66.

Ashida, G., Wagner, H., and Carr, C.E. (2010). Processing of Phase-Locked Spikes and Periodic Signals. In Analysis of Parallel Spike Trains, S. Grün, and S. Rotter, eds. (Boston, MA: Springer US), pp. 59-74.

Avissar, M., Wittig, J.H., Saunders, J.C., and Parsons, T.D. (2013). Refractoriness enhances temporal coding by auditory nerve fibers. J Neurosci 33, 7681-7690.

Ayala, Y.A., and Malmierca, M.S. (2012). Stimulus-specific adaptation and deviance detection in the inferior colliculus. Front Neural Circuits 6, 89.

Azim, E., Jiang, J., Alstermark, B., and Jessell, T.M. (2014). Skilled reaching relies on a V2a propriospinal internal copy circuit. Nature 508, 357-363.

Barnéoud, P., and Van der Loos, H. (1993). Direction of handedness linked to hereditary asymmetry of a sensory system. Proc Natl Acad Sci U S A 90, 3246-3250.

Barres, B.A., and Raff, M.C. (1993). Proliferation of oligodendrocyte precursor cells depends on electrical activity in axons. Nature 361, 258-260.

Barres, B.A., Chun, L.L., and Corey, D.P. (1988). Ion channel expression by white matter glia: I. Type 2 astrocytes and oligodendrocytes. Glia 1, 10-30.

Barres, B.A., Chun, L.L., and Corey, D.P. (1990). Ion channels in vertebrate glia. Annu Rev Neurosci 13, 441-474. 
Barrett, E.F., and Barrett, J.N. (1982). Intracellular recording from vertebrate myelinated axons: mechanism of the depolarizing afterpotential. J Physiol (Lond) 323, 117-144.

Bartlett, E.L., and Wang, X. (2005). Long-lasting modulation by stimulus context in primate auditory cortex. J Neurophysiol 94, 83-104.

Bartzokis, G. (2004). Age-related myelin breakdown: a developmental model of cognitive decline and Alzheimer's disease. Neurobiol Aging 25, 5-18.

Bayazitov, I.T., Westmoreland, J.J., and Zakharenko, S.S. (2013). Forward suppression in the auditory cortex is caused by the $\mathrm{Ca}(\mathrm{v}) 3.1$ calcium channel-mediated switch from bursting to tonic firing at thalamocortical projections. J Neurosci 33, 18940-18950.

Bechler, M.E., Byrne, L., and Ffrench-Constant, C. (2015). CNS myelin sheath lengths are an intrinsic property of oligodendrocytes. Curr Biol 25, 2411-2416.

Beirowski, B. (2013). Concepts for regulation of axon integrity by enwrapping glia. Front Cell Neurosci 7, 256.

Bellesi, M., Haswell, J.D., de Vivo, L., Marshall, W., Roseboom, P.H., Tononi, G., and Cirelli, C. (2018). Myelin modifications after chronic sleep loss in adolescent mice. Sleep.

Bercury, K.K., and Macklin, W.B. (2015). Dynamics and mechanisms of CNS myelination. Dev Cell 32, 447-458.

Berker, E.A. (1986). Translation of broca's 1865 report. Arch Neurol 43, 1065.

Berthold, C.H., Fabricius, C., Rydmark, M., and Andersén, B. (1993). Axoplasmic organelles at nodes of Ranvier. I. Occurrence and distribution in large myelinated spinal root axons of the adult cat. $J$ Neurocytol 22, 925-940.

Beukes, E.W., Munro, K.J., and Purdy, S.C. (2009). Duration-sensitive neurons in the auditory cortex. Neuroreport 20, 1129-1133.

Biddle, F.G., Coffaro, C.M., Ziehr, J.E., and Eales, B.A. (1993). Genetic variation in paw preference (handedness) in the mouse. Genome 36, 935-943.

Boggs, J.M. (2006). Myelin basic protein: a multifunctional protein. Cell Mol Life Sci 63, 1945-1961.

Boiko, T., Rasband, M.N., Levinson, S.R., Caldwell, J.H., Mandel, G., Trimmer, J.S., and Matthews, G. (2001). Compact myelin dictates the differential targeting of two sodium channel isoforms in the same axon. Neuron 30, 91-104.

Brady, S.T., Witt, A.S., Kirkpatrick, L.L., de Waegh, S.M., Readhead, C., Tu, P.H., and Lee, V.M. (1999). Formation of compact myelin is required for maturation of the axonal cytoskeleton. J Neurosci 19, 7278-7288.

Bregman, A.S. (1994). Auditory scene analysis: The perceptual organization of sound (Cambridge, Mass: MIT Press).

Brown, A.M., Tekkök, S.B., and Ransom, B.R. (2003). Glycogen regulation and functional role in mouse white matter. J Physiol (Lond) 549, 501-512. 
Bruggemann, J.M., Stockill, H.V., Lenroot, R.K., and Laurens, K.R. (2013). Mismatch negativity (MMN) and sensory auditory processing in children aged 9-12 years presenting with putative antecedents of schizophrenia. Int J Psychophysiol 89, 374-380.

Brzózka, M.M., Rossner, M.J., and de Hoz, L. (2016). Tcf4 transgenic female mice display delayed adaptation in an auditory latent inhibition paradigm. Eur Arch Psychiatry Clin Neurosci 266, 505-512.

Bullock, A.E., Slobe, B.S., Vázquez, V., and Collins, A.C. (1997). Inbred mouse strains differ in the regulation of startle and prepulse inhibition of the startle response. Behav Neurosci 111, 1353-1360.

Burger, R.M., and Pollak, G.D. (1998). Analysis of the role of inhibition in shaping responses to sinusoidally amplitude-modulated signals in the inferior colliculus. J Neurophysiol 80, 1686-1701.

Buzsáki, G., and Wang, X.-J. (2012). Mechanisms of gamma oscillations. Annu Rev Neurosci 35, 203225.

Carandini, M., and Ferster, D. (1997). A tonic hyperpolarization underlying contrast adaptation in cat visual cortex. Science 276, 949-952.

Carmody, D.P., and Lewis, M. (2010). Regional white matter development in children with autism spectrum disorders. Dev Psychobiol 52, 755-763.

Carpinelli, M.R., Voss, A.K., Manning, M.G., Perera, A.A., Cooray, A.A., Kile, B.T., and Burt, R.A. (2014). A new mouse model of Canavan leukodystrophy displays hearing impairment due to central nervous system dysmyelination. Dis Model Mech 7, 649-657.

Carr, C.E., Soares, D., Parameshwaran, S., and Perney, T. (2001). Evolution and development of time coding systems. Curr Opin Neurobiol 11, 727-733.

Cassells, B., Collins, R.L., and Wahlsten, D. (1990). Path analysis of sex difference, forebrain commissure area and brain size in relation to degree of laterality in selectively bred mice. Brain Res $529,50-56$.

Chang, E.F., Bao, S., Imaizumi, K., Schreiner, C.E., and Merzenich, M.M. (2005). Development of spectral and temporal response selectivity in the auditory cortex. Proc Natl Acad Sci U S A 102, 16460-16465.

Chao, L.L., Tosun, D., Woodward, S.H., Kaufer, D., and Neylan, T.C. (2015). Preliminary Evidence of Increased Hippocampal Myelin Content in Veterans with Posttraumatic Stress Disorder. Front Behav Neurosci 9, 333.

Chernoff, G.F. (1981). Shiverer: an autosomal recessive mutant mouse with myelin deficiency. J Hered $72,128$.

Chesnut, T.J., and Swann, J.W. (1990). Suppression of 4-aminopyridine-induced epileptogenesis by the GABAA agonist muscimol. Epilepsy Res 5, 8-17.

Chiappa, K.H., Harrison, J.L., Brooks, E.B., and Young, R.R. (1980). Brainstem auditory evoked responses in 200 patients with multiple sclerosis. Ann Neurol 7, 135-143.

Chiappelli, J., Hong, L.E., Wijtenburg, S.A., Du, X., Gaston, F., Kochunov, P., and Rowland, L.M. (2015). Alterations in frontal white matter neurochemistry and microstructure in schizophrenia: implications for neuroinflammation. Translational Psychiatry 5, e548. 
Clark, K.C., Josephson, A., Benusa, S.D., Hartley, R.K., Baer, M., Thummala, S., Joslyn, M., Sword, B.A., Elford, H., Oh, U., et al. (2016). Compromised axon initial segment integrity in EAE is preceded by microglial reactivity and contact. Glia 64, 1190-1209.

Clark, M.G., Sherman, G.F., Bimonte, H.A., and Fitch, R.H. (2000). Perceptual auditory gap detection deficits in male BXSB mice with cerebrocortical ectopias. Neuroreport 11, 693-696.

Clarkson, C., Antunes, F.M., and Rubio, M.E. (2016). Conductive Hearing Loss Has Long-Lasting Structural and Molecular Effects on Presynaptic and Postsynaptic Structures of Auditory Nerve Synapses in the Cochlear Nucleus. J Neurosci 36, 10214-10227.

Collins, R.L. (1968). On the Inheritance of Handedness (I. Laterality in inbread mice). The Journal of Heredity.

Collins, R.L., Sargent, E.E., and Neumann, P.E. (1993). Genetic and behavioral tests of the McManus hypothesis relating response to selection for lateralization of handedness in mice to degree of heterozygosity. Behav Genet 23, 413-421.

Cornish, K.M., and McManus, I.C. (1996). Hand preference and hand skill in children with autism. J Autism Dev Disord 26, 597-609.

Creutzfeldt, O., Hellweg, F.C., and Schreiner, C. (1980). Thalamocortical transformation of responses to complex auditory stimuli. Exp Brain Res 39, 87-104.

Das, A. (1997). Plasticity in adult sensory cortex: a review. Network: Computation in Neural Systems 8 , R33-R76.

Davis, M., Gendelman, D.S., Tischler, M.D., and Gendelman, P.M. (1982). A primary acoustic startle circuit: lesion and stimulation studies. J Neurosci 2, 791-805.

Dehmel, S., Eisinger, D., and Shore, S.E. (2012). Gap prepulse inhibition and auditory brainstemevoked potentials as objective measures for tinnitus in guinea pigs. Front Syst Neurosci 6, 42.

Demerens, C., Stankoff, B., Logak, M., Anglade, P., Allinquant, B., Couraud, F., Zalc, B., and Lubetzki, C. (1996). Induction of myelination in the central nervous system by electrical activity. Proc Natl Acad Sci U S A 93, 9887-9892.

Dimou, L., Simon, C., Kirchhoff, F., Takebayashi, H., and Götz, M. (2008). Progeny of Olig2-expressing progenitors in the gray and white matter of the adult mouse cerebral cortex. J Neurosci 28, 1043410442.

Dollfus, S., Alary, M., Razafimandimby, A., Prelipceanu, D., Rybakowski, J.K., Davidson, M., Galderisi, S., Libiger, J., Hranov, L.G., Hummer, M., et al. (2012). Familial sinistrality and handedness in patients with first episode schizophrenia: the EUFEST study. Laterality 17, 217-224.

Domènech-Estévez, E., Baloui, H., Repond, C., Rosafio, K., Médard, J.-J., Tricaud, N., Pellerin, L., and Chrast, R. (2015). Distribution of monocarboxylate transporters in the peripheral nervous system suggests putative roles in lactate shuttling and myelination. J Neurosci 35, 4151-4156.

Dreyer, A., and Delgutte, B. (2006). Phase locking of auditory-nerve fibers to the envelopes of highfrequency sounds: implications for sound localization. J Neurophysiol 96, 2327-2341. 
Dyakin, V.V., Chen, Y., Branch, C.A., Veeranna, Yuan, A., Rao, M., Kumar, A., Peterhoff, C.M., and Nixon, R.A. (2010). The contributions of myelin and axonal caliber to transverse relaxation time in shiverer and neurofilament-deficient mouse models. Neuroimage 51, 1098-1105.

Eddins, D.A., and Green, D.M. (1995). Temporal Integration and Temporal resolution . In Hearing , B.C.J. Moore, ed. (United States of America: Academic Press), pp. 207-242.

Edgar, J.M., McLaughlin, M., Werner, H.B., McCulloch, M.C., Barrie, J.A., Brown, A., Faichney, A.B., Snaidero, N., Nave, K.-A., and Griffiths, I.R. (2009). Early ultrastructural defects of axons and axon-glia junctions in mice lacking expression of Cnp1. Glia 57, 1815-1824.

Eggermont, J.J. (1999). Neural correlates of gap detection in three auditory cortical fields in the Cat. J Neurophysiol 81, 2570-2581.

Eggermont, J.J. (2015). Auditory temporal processing and its disorders (Oxford University Press).

Eggermont, J.J., and Wang, X. (2011). Temporal coding in auditory cortex. In The Auditory Cortex, J.A. Winer, and C.E. Schreiner, eds. (Boston, MA: Springer US), pp. 309-328.

Einheber, S., Bhat, M.A., and Salzer, J.L. (2006). Disrupted axo-glial junctions result in accumulation of abnormal mitochondria at nodes of ranvier. Neuron Glia Biol 2, 165-174.

El-Badry, M.M., Ding, D., McFadden, S.L., and Eddins, A.C. (2007). Physiological effects of auditory nerve myelinopathy in chinchillas. Eur J Neurosci 25, 1437-1446.

Ellens, D.J., Gaidica, M., Toader, A., Peng, S., Shue, S., John, T., Bova, A., and Leventhal, D.K. (2016). An automated rat single pellet reaching system with high-speed video capture. J Neurosci Methods 271, 119-127.

Engel, M., Snikeris, P., Jenner, A., Karl, T., Huang, X.-F., and Frank, E. (2015). Neuregulin 1 Prevents Phencyclidine-Induced' ' Behavioral Impairments and Disruptions to GABAergic Signaling in Mice. Int J Neuropsychopharmacol 18, pyu114.

Ettle, B., Schlachetzki, J.C.M., and Winkler, J. (2016). Oligodendroglia and myelin in neurodegenerative diseases: more than just bystanders? Mol Neurobiol 53, 3046-3062.

Evans, M.D., Dumitrescu, A.S., Kruijssen, D.L.H., Taylor, S.E., and Grubb, M.S. (2015). Rapid modulation of axon initial segment length influences repetitive spike firing. Cell Rep 13, 1233-1245.

Fabricius, C., Berthold, C.H., and Rydmark, M. (1993). Axoplasmic organelles at nodes of Ranvier. II. Occurrence and distribution in large myelinated spinal cord axons of the adult cat. J Neurocytol 22, 941-954.

Feng, Y., Wang, J., and Yin, S. (2007). General anesthesia changes gap-evoked auditory responses in guinea pigs. Acta Otolaryngol 127, 143-148.

Fields, R.D. (2008a). White matter in learning, cognition and psychiatric disorders. Trends Neurosci 31, 361-370.

Fields, R.D. (2008b). Oligodendrocytes changing the rules: action potentials in glia and oligodendrocytes controlling action potentials. Neuroscientist 14, 540-543. 
Fields, R.D., and Burnstock, G. (2006). Purinergic signalling in neuron-glia interactions. Nat Rev Neurosci 7, 423-436.

Fingher, N., Dinstein, I., Ben-Shachar, M., Haar, S., Dale, A.M., Eyler, L., Pierce, K., and Courchesne, E. (2017). Toddlers later diagnosed with autism exhibit multiple structural abnormalities in temporal corpus callosum fibers. Cortex 97, 291-305.

Ford, M.C., Alexandrova, O., Cossell, L., Stange-Marten, A., Sinclair, J., Kopp-Scheinpflug, C., Pecka, M., Attwell, D., and Grothe, B. (2015). Tuning of Ranvier node and internode properties in myelinated axons to adjust action potential timing. Nat Commun 6, 8073.

Friede, R.L., and Bischhausen, R. (1982). How are sheath dimensions affected by axon caliber and internode length? Brain Res 235, 335-350.

Friedman, J.T., Peiffer, A.M., Clark, M.G., Benasich, A.A., and Fitch, R.H. (2004). Age and experiencerelated improvements in gap detection in the rat. Brain Res Dev Brain Res 152, 83-91.

Friedrich, P., Ocklenburg, S., Heins, N., Schlüter, C., Fraenz, C., Beste, C., Güntürkün, O., and Genç, E. (2017). Callosal microstructure affects the timing of electrophysiological left-right differences. Neuroimage 163, 310-318.

Frisina, R.D. (2001). Subcortical neural coding mechanisms for auditory temporal processing. Hear Res 158,1-27.

Frühbeis, C., Fröhlich, D., Kuo, W.P., Amphornrat, J., Thilemann, S., Saab, A.S., Kirchhoff, F., Möbius, W., Goebbels, S., Nave, K.-A., et al. (2013). Neurotransmitter-triggered transfer of exosomes mediates oligodendrocyte-neuron communication. PLoS Biol 11, e1001604.

Fünfschilling, U., Supplie, L.M., Mahad, D., Boretius, S., Saab, A.S., Edgar, J., Brinkmann, B.G., Kassmann, C.M., Tzvetanova, I.D., Möbius, W., et al. (2012). Glycolytic oligodendrocytes maintain myelin and long-term axonal integrity. Nature 485, 517-521.

Gabbard, C., and Hart, S. (1996). A question of foot dominance. J Gen Psychol 123, 289-296.

Gazzaniga, M.S. (2000). Cerebral specialization and interhemispheric communication: does the corpus callosum enable the human condition? Brain 123 ( Pt 7), 1293-1326.

Ge, Y., Wu, J., Sun, X., and Zhang, K. (2011). Enhanced mismatch negativity in adolescents with posttraumatic stress disorder (PTSD). Int J Psychophysiol 79, 231-235.

Ghosh, A., Manrique-Hoyos, N., Voigt, A., Schulz, J.B., Kreutzfeldt, M., Merkler, D., and Simons, M. (2011). Targeted ablation of oligodendrocytes triggers axonal damage. PLoS ONE 6, e22735.

Gibson, E.M., Purger, D., Mount, C.W., Goldstein, A.K., Lin, G.L., Wood, L.S., Inema, I., Miller, S.E., Bieri, G., Zuchero, J.B., et al. (2014). Neuronal activity promotes oligodendrogenesis and adaptive myelination in the mammalian brain. Science 344, 1252304.

Gilbert, C.D., and Li, W. (2012). Adult visual cortical plasticity. Neuron 75, 250-264.

Giljov, A., Karenina, K., Ingram, J., and Malashichev, Y. (2015). Parallel emergence of true handedness in the evolution of marsupials and placentals. Curr Biol 25, 1878-1884. 
Gillberg, C. (1983). Autistic children's hand preferences: results from an epidemiological study of infantile autism. Psychiatry Res 10, 21-30.

Goldstein Jr., M.H., Kiang, N.Y.-S., and Brown, R.M. (1959). Responses of the Auditory Cortex to Repetitive Acoustic Stimuli. J Acoust Soc Am 31, 356-364.

Gollisch, T., and Herz, A.V.M. (2004). Input-driven components of spike-frequency adaptation can be unmasked in vivo. J Neurosci 24, 7435-7444.

Gorski, J.A., Talley, T., Quu, M., Puelles, L., Rubenstein, J.L.R., and Jones, K.R. (2002). Cortical excitatory neurons and glia, but not GABAergic neurons, are produced in the Emx1-expressing lineage. J Neurosci 22, 6309-6314.

Griffiths, I., Klugmann, M., Anderson, T., Yool, D., Thomson, C., Schwab, M.H., Schneider, A., Zimmermann, F., McCulloch, M., Nadon, N., et al. (1998). Axonal swellings and degeneration in mice lacking the major proteolipid of myelin. Science 280, 1610-1613.

Grubb, M.S., and Burrone, J. (2010). Activity-dependent relocation of the axon initial segment finetunes neuronal excitability. Nature 465, 1070-1074.

Gruber, D., Waanders, R., Collins, R.L., Wolfer, D.P., and Lipp, H.P. (1991). Weak or missing paw lateralization in a mouse strain (I/LnJ) with congenital absence of the corpus callosum. Behav Brain Res 46, 9-16.

Gulisano, M., Broccoli, V., Pardini, C., and Boncinelli, E. (1996). Emx1 and Emx2 show different patterns of expression during proliferation and differentiation of the developing cerebral cortex in the mouse. Eur J Neurosci 8, 1037-1050.

Guo, J.-Z., Graves, A.R., Guo, W.W., Zheng, J., Lee, A., Rodríguez-González, J., Li, N., Macklin, J.J., Phillips, J.W., Mensh, B.D., et al. (2015). Cortex commands the performance of skilled movement. Elife 4, e10774.

Gutzmann, A., Ergül, N., Grossmann, R., Schultz, C., Wahle, P., and Engelhardt, M. (2014). A period of structural plasticity at the axon initial segment in developing visual cortex. Front Neuroanat $8,11$.

Háden, G.P., Honing, H., Török, M., and Winkler, I. (2015). Detecting the temporal structure of sound sequences in newborn infants. Int J Psychophysiol 96, 23-28.

Halestrap, A.P. (2012). The monocarboxylate transporter family--Structure and functional characterization. IUBMB Life 64, 1-9.

Hamada, M.S., and Kole, M.H.P. (2015). Myelin loss and axonal ion channel adaptations associated with gray matter neuronal hyperexcitability. J Neurosci 35, 7272-7286.

Hamann, I., Gleich, O., Klump, G.M., Kittel, M.C., and Strutz, J. (2004). Age-dependent changes of gap detection in the Mongolian gerbil (Meriones unguiculatus). J Assoc Res Otolaryngol 5, 49-57.

Haroutunian, V., Katsel, P., Roussos, P., Davis, K.L., Altshuler, L.L., and Bartzokis, G. (2014). Myelination, oligodendrocytes, and serious mental illness. Glia 62, 1856-1877.

Haß, K., Bak, N., Szycik, G.R., Glenthøj, B.Y., and Oranje, B. (2017). Deficient prepulse inhibition of the startle reflex in schizophrenia using a cross-modal paradigm. Biol Psychol 128, 112-116. 
Hautus, M.J., Setchell, G.J., Waldie, K.E., and Kirk, I.J. (2003). Age-related improvements in auditory temporal resolution in reading-impaired children. Dyslexia 9, 37-45.

Hellmann, M.A., Steiner, I., and Mosberg-Galili, R. (2011). Sudden sensorineural hearing loss in multiple sclerosis: clinical course and possible pathogenesis. Acta Neurol Scand 124, 245-249.

Hendler, T., Squires, N.K., and Emmerich, D.S. (1990). Psychophysical Measures of CentralAuditory Dysfunction in Multiple Sclerosis: Neurophysiological and Neuroanatomical Correlates. Ear Hear 11, 403-416.

Henneke, M., Gegner, S., Hahn, A., Plecko-Startinig, B., Weschke, B., Gärtner, J., and Brockmann, K. (2010). Clinical neurophysiology in GJA12-related hypomyelination vs Pelizaeus-Merzbacher disease. Neurology 74, 1785-1789.

Hildebrand, C., and Hahn, R. (1978). Relation between myelin sheath thickness and axon size in spinal cord white matter of some vertebrate species. J Neurol Sci 38, 421-434.

Hildebrand, C., Remahl, S., Persson, H., and Bjartmar, C. (1993). Myelinated nerve fibres in the CNS. Prog Neurobiol 40, 319-384.

Hira, R., Terada, S.-I., Kondo, M., and Matsuzaki, M. (2015). Distinct functional modules for discrete and rhythmic forelimb movements in the mouse motor cortex. J Neurosci 35, 13311-13322.

Hollenbeck, P.J., and Saxton, W.M. (2005). The axonal transport of mitochondria. J Cell Sci 118, 5411-5419.

Hoover, E., Pasquesi, L., and Souza, P. (2015). Comparison of clinical and traditional gap detection tests. J Am Acad Audiol 26, 540-546.

De Hoz, L., and Nelken, I. (2014). Frequency tuning in the behaving mouse: different bandwidths for discrimination and generalization. PLoS ONE 9, e91676.

De Hoz, L., and Simons, M. (2015). The emerging functions of oligodendrocytes in regulating neuronal network behaviour. Bioessays 37, 60-69.

De Hoz, L., Gierej, D., Lioudyno, V., Jaworski, J., Blazejczyk, M., Cruces-Solís, H., Beroun, A., Lebitko, T., Nikolaev, T., Knapska, E., et al. (2017). Blocking c-Fos Expression Reveals the Role of Auditory Cortex Plasticity in Sound Frequency Discrimination Learning. Cereb Cortex 1-11.

Huchzermeyer, C., Berndt, N., Holzhütter, H.-G., and Kann, O. (2013). Oxygen consumption rates during three different neuronal activity states in the hippocampal CA3 network. J Cereb Blood Flow Metab 33, 263-271.

Hughes, E.G., Orthmann-Murphy, J.L., Langseth, A.J., Bergles, D.E., Hughes, E., Orthmann-Murphy, J., Langseth, A., Bergles, D., Hughes, E., Orthmann-Murphy, J., et al. (2018). Myelin remodeling through experience-dependent oligodendrogenesis in the adult somatosensory cortex. Nat Neurosci.

Imaizumi, K., and Lee, C.C. (2015). Auditory Thalamocortical Transformations. In Encyclopedia of Computational Neuroscience, D. Jaeger, and R. Jung, eds. (New York, NY: Springer New York), pp. 278-292. 
Inoue, Y., Nakamura, R., Mikoshiba, K., and Tsukada, Y. (1981). Fine structure of the central myelin sheath in the myelin deficient mutant Shiverer mouse, with special reference to the pattern of myelin formation by oligodendroglia. Brain Res 219, 85-94.

International Mouse Knockout Consortium, Collins, F.S., Rossant, J., and Wurst, W. (2007). A mouse for all reasons. Cell 128, 9-13.

Irwin, R.J., Hinchcliff, L.K., and Kemp, S. (1981). Temporal acuity in normal and hearing-impaired listeners. Audiology 20, 234-243.

Iscru, E., Serinagaoglu, Y., Schilling, K., Tian, J., Bowers-Kidder, S.L., Zhang, R., Morgan, J.I., DeVries, A.C., Nelson, R.J., Zhu, M.X., et al. (2009). Sensorimotor enhancement in mouse mutants lacking the Purkinje cell-specific Gi/o modulator, Pcp2(L7). Mol Cell Neurosci 40, 62-75.

Ison, J.R., Castro, J., Allen, P., Virag, T.M., and Walton, J.P. (2002). The relative detectability for mice of gaps having different ramp durations at their onset and offset boundaries. J Acoust Soc Am 112, 740-747.

Ito, T., Tokuriki, M., Shibamori, Y., Saito, T., and Nojyo, Y. (2004). Cochlear nerve demyelination causes prolongation of wave I latency in ABR of the myelin deficient (md) rat. Hear Res 191, 119-124.

Jamann, N., Jordan, M., and Engelhardt, M. (2018). Activity-dependent axonal plasticity in sensory systems. Neuroscience 368, 268-282.

Jean, P., Lopez de la Morena, D., Michanski, S., Jaime Tobón, L.M., Chakrabarti, R., Picher, M.M., Neef, J., Jung, S., Gültas, M., Maxeiner, S., et al. (2018). The synaptic ribbon is critical for sound encoding at high rates and with temporal precision. Elife 7.

Jin, Z., Liang, G.-H., Cooper, E.C., and Jarlebark, L. (2009). Expression and localization of K channels KCNQ2 and KCNQ3 in the mammalian cochlea. Audiol Neurootol 14, 98-105.

Joachimsthaler, B., Uhlmann, M., Miller, F., Ehret, G., and Kurt, S. (2014). Quantitative analysis of neuronal response properties in primary and higher-order auditory cortical fields of awake house mice (Mus musculus). Eur J Neurosci 39, 904-918.

Jones, S.L., and Svitkina, T.M. (2016). Axon initial segment cytoskeleton: architecture, development, and role in neuron polarity. Neural Plast 2016, 6808293.

Joris, P.X., Schreiner, C.E., and Rees, A. (2004). Neural processing of amplitude-modulated sounds. Physiol Rev 84, 541-577.

Ju, H., Hines, M.L., and Yu, Y. (2016). Cable energy function of cortical axons. Sci Rep 6, 29686.

Jung, J., Morlet, D., Mercier, B., Confavreux, C., and Fischer, C. (2006). Mismatch negativity (MMN) in multiple sclerosis: an event-related potentials study in 46 patients. Clin Neurophysiol 117, 85-93.

Jung, S., Maritzen, T., Wichmann, C., Jing, Z., Neef, A., Revelo, N.H., Al-Moyed, H., Meese, S., Wojcik, S.M., Panou, I., et al. (2015). Disruption of adaptor protein $2 \mu($ AP- $2 \mu)$ in cochlear hair cells impairs vesicle reloading of synaptic release sites and hearing. EMBO J 34, 2686-2702.

Kann, O. (2016). The interneuron energy hypothesis: Implications for brain disease. Neurobiol Dis 90, 75-85. 
Kann, O., Huchzermeyer, C., Kovács, R., Wirtz, S., and Schuelke, M. (2011). Gamma oscillations in the hippocampus require high complex I gene expression and strong functional performance of mitochondria. Brain 134, 345-358.

Kann, O., Papageorgiou, I.E., and Draguhn, A. (2014). Highly energized inhibitory interneurons are a central element for information processing in cortical networks. J Cereb Blood Flow Metab 34, 1270 1282.

Kanzaki, J., Mikoshiba, K., and Tsukada, Y. (1985). Auditory brain stem response in neuropathological mutant mice (shiverer and reeler). ORL J Otorhinolaryngol Relat Spec 47, $294-298$.

Káradóttir, R., Cavelier, P., Bergersen, L.H., and Attwell, D. (2005). NMDA receptors are expressed in oligodendrocytes and activated in ischaemia. Nature 438, 1162-1166.

Karlen, S.J., and Krubitzer, L. (2007). The functional and anatomical organization of marsupial neocortex: evidence for parallel evolution across mammals. Prog Neurobiol 82, 122-141.

Karlsson, U., and Schultz, R.L. (1965). Fixation of the central nervous system for electron microscopy by aldehyde perfusion. J Ultrastruct Res 12, 160-186.

Keller, C.H., Kaylegian, K., and Wehr, M. (2018). Gap encoding by parvalbumin-expressing interneurons in auditory cortex. J Neurophysiol.

Kettenmann, H., Gilbert, P., and Schachner, M. (1984). Depolarization of cultured oligodendrocytes by glutamate and GABA. Neurosci Lett 47, 271-276.

Kikusui, T., Kiyokawa, Y., and Mori, Y. (2007). Deprivation of mother-pup interaction by early weaning alters myelin formation in male, but not female, ICR mice. Brain Res 1133, 115-122.

Kim, J.H., Renden, R., and von Gersdorff, H. (2013a). Dysmyelination of auditory afferent axons increases the jitter of action potential timing during high-frequency firing. J Neurosci 33, 9402-9407.

Kim, S.E., Turkington, K., Kushmerick, C., and Kim, J.H. (2013b). Central dysmyelination reduces the temporal fidelity of synaptic transmission and the reliability of postsynaptic firing during high-frequency stimulation. J Neurophysiol 110, 1621-1630.

Kimura, F., and Itami, C. (2009). Myelination and isochronicity in neural networks. Front Neuroanat 3, 12.

Kimura, M., Inoko, H., Katsuki, M., Ando, A., Sato, T., Hirose, T., Takashima, H., Inayama, S., Okano, H., and Takamatsu, K. (1985). Molecular genetic analysis of myelin-deficient mice: shiverer mutant mice show deletion in gene(s) coding for myelin basic protein. J Neurochem 44, 692-696.

Kirkpatrick, L.L., Witt, A.S., Payne, H.R., Shine, H.D., and Brady, S.T. (2001). Changes in microtubule stability and density in myelin-deficient shiverer mouse CNS axons. J Neurosci 21, 2288-2297.

Kirschner, D.A., and Ganser, A.L. (1980). Compact myelin exists in the absence of basic protein in the shiverer mutant mouse. Nature 283, 207-210.

Van der Knaap, L.J., and van der Ham, I.J.M. (2011). How does the corpus callosum mediate interhemispheric transfer? A review. Behav Brain Res 223, 211-221.

Koch, M. (1999). The neurobiology of startle. Prog Neurobiol 59, 107-128. 
Kole, M.H.P., and Stuart, G.J. (2012). Signal processing in the axon initial segment. Neuron 73, 235247.

Köppl, C. (1997). Phase locking to high frequencies in the auditory nerve and cochlear nucleus magnocellularis of the barn owl, Tyto alba. J Neurosci 17, 3312-3321.

Kuan, C.-C., Sano, M., Kaga, K., Kodama, M., and Kodama, K. (2008). Hearing profile and MRI myelination of auditory pathway in Pelizaeus-Merzbacher disease. Acta Otolaryngol 128, 539-546.

Kuba, H., Adachi, R., and Ohmori, H. (2014). Activity-dependent and activity-independent development of the axon initial segment. J Neurosci 34, 3443-3453.

Kujawa, S.G., and Liberman, M.C. (2009). Adding insult to injury: cochlear nerve degeneration after "temporary" noise-induced hearing loss. J Neurosci 29, 14077-14085.

Kutzelnigg, A., Lucchinetti, C.F., Stadelmann, C., Brück, W., Rauschka, H., Bergmann, M., Schmidbauer, M., Parisi, J.E., and Lassmann, H. (2005). Cortical demyelination and diffuse white matter injury in multiple sclerosis. Brain 128, 2705-2712.

Kwiecien, J.M., O'Connor, L.T., Goetz, B.D., Delaney, K.H., Fletch, A.L., and Duncan, I.D. (1998). Morphological and morphometric studies of the dysmyelinating mutant, the Long Evans shaker rat. $J$ Neurocytol 27, 581-591.

Lai, S., Panarese, A., Spalletti, C., Alia, C., Ghionzoli, A., Caleo, M., and Micera, S. (2015). Quantitative kinematic characterization of reaching impairments in mice after a stroke. Neurorehabil Neural Repair 29, 382-392.

Lakhani, B., Borich, M.R., Jackson, J.N., Wadden, K.P., Peters, S., Villamayor, A., MacKay, A.L., Vavasour, I.M., Rauscher, A., and Boyd, L.A. (2016). Motor skill acquisition promotes human brain myelin plasticity. Neural Plast 2016, 7526135.

Land, R., Burghard, A., and Kral, A. (2016). The contribution of inferior colliculus activity to the auditory brainstem response (ABR) in mice. Hear Res 341, 109-118.

Lang, E.J., and Rosenbluth, J. (2003). Role of myelination in the development of a uniform olivocerebellar conduction time. J Neurophysiol 89, 2259-2270.

Lappe-Siefke, C., Goebbels, S., Gravel, M., Nicksch, E., Lee, J., Braun, P.E., Griffiths, I.R., and Nave, K.-A. (2003). Disruption of Cnp1 uncouples oligodendroglial functions in axonal support and myelination. Nat Genet 33, 366-374.

Lasiene, J., Matsui, A., Sawa, Y., Wong, F., and Horner, P.J. (2009). Age-related myelin dynamics revealed by increased oligodendrogenesis and short internodes. Aging Cell 8, 201-213.

Lasky, R.E., Soto, A.A., Luck, M.L., and Laughlin, N.K. (1999). Otoacoustic emission, evoked potential, and behavioral auditory thresholds in the rhesus monkey (Macaca mulatta). Hear Res 136, 35-43.

Lee, Y., López, D.E., Meloni, E.G., and Davis, M. (1996). A primary acoustic startle pathway: obligatory role of cochlear root neurons and the nucleus reticularis pontis caudalis. J Neurosci 16, 3775-3789.

Lee, Y., Morrison, B.M., Li, Y., Lengacher, S., Farah, M.H., Hoffman, P.N., Liu, Y., Tsingalia, A., Jin, L., Zhang, P.-W., et al. (2012). Oligodendroglia metabolically support axons and contribute to neurodegeneration. Nature 487, 443-448. 
Lehmann, M.L., Weigel, T.K., Elkahloun, A.G., and Herkenham, M. (2017). Chronic social defeat reduces myelination in the mouse medial prefrontal cortex. Sci Rep 7, 46548.

Leitner, D.S., Hammond, G.R., Springer, C.P., Ingham, K.M., Mekilo, A.M., Bodison, P.R., Aranda, M.T., and Shawaryn, M.A. (1993). Parameters affecting gap detection in the rat. Percept Psychophys 54, 395-405.

Leone, D.P., Genoud, S., Atanasoski, S., Grausenburger, R., Berger, P., Metzger, D., Macklin, W.B., Chambon, P., and Suter, U. (2003). Tamoxifen-inducible glia-specific Cre mice for somatic mutagenesis in oligodendrocytes and Schwann cells. Mol Cell Neurosci 22, 430-440.

Leterrier, C., Clerc, N., Rueda-Boroni, F., Montersino, A., Dargent, B., and Castets, F. (2017). Ankyrin $\mathrm{G}$ membrane partners drive the establishment and maintenance of the axon initial segment. Front Cell Neurosci 11, 6 .

Leuzzi, V., Rinna, A., Gallucci, M., Di Capua, M., Dionisi-Vici, C., Longo, D., and Bertini, E. (2000). Ataxia, deafness, leukodystrophy: inherited disorder of the white matter in three related patients. Neurology 54, 2325-2328.

Lewis, M.S., Lilly, D.J., Hutter, M.M., Bourdette, D.N., McMillan, G.P., Fitzpatrick, M.A., and Fausti, S.A. (2010). Audiometric hearing status of individuals with and without multiple sclerosis. The Journal of Rehabilitation Research and Development 47, 669.

Li, L., Lei, D., Li, L., Huang, X., Suo, X., Xiao, F., Kuang, W., Li, J., Bi, F., Lui, S., et al. (2016). White Matter Abnormalities in Post-traumatic Stress Disorder Following a Specific Traumatic Event. EBioMedicine 4, 176-183.

Li, Y.-C., Zhai, X.-Y., Ohsato, K., Futamata, H., Shimada, O., and Atsumi, S. (2004). Mitochondrial accumulation in the distal part of the initial segment of chicken spinal motoneurons. Brain Res 1026, 235-243.

Liu, J., Dietz, K., DeLoyht, J.M., Pedre, X., Kelkar, D., Kaur, J., Vialou, V., Lobo, M.K., Dietz, D.M., Nestler, E.J., et al. (2012). Impaired adult myelination in the prefrontal cortex of socially isolated mice. Nat Neurosci 15, 1621-1623.

Liu, L.-F., Palmer, A.R., and Wallace, M.N. (2006). Phase-locked responses to pure tones in the inferior colliculus. J Neurophysiol 95, 1926-1935.

Lodygensky, G.A., Marques, J.P., Maddage, R., Perroud, E., Sizonenko, S.V., Hüppi, P.S., and Gruetter, R. (2012). In vivo assessment of myelination by phase imaging at high magnetic field. Neuroimage 59, 1979-1987.

Long, P., Wan, G., Roberts, M.T., and Corfas, G. (2018). Myelin development, plasticity, and pathology in the auditory system. Dev Neurobiol 78, 80-92.

Lopez-Poveda, E.A., and Barrios, P. (2013). Perception of stochastically undersampled sound waveforms: a model of auditory deafferentation. Front Neurosci 7, 124.

Lutas, A., Birnbaumer, L., and Yellen, G. (2014). Metabolism regulates the spontaneous firing of substantia nigra pars reticulata neurons via KATP and nonselective cation channels. J Neurosci 34 , 16336-16347. 
Mächler, P., Wyss, M.T., Elsayed, M., Stobart, J., Gutierrez, R., von Faber-Castell, A., Kaelin, V., Zuend, M., San Martín, A., Romero-Gómez, I., et al. (2016). In Vivo Evidence for a Lactate Gradient from Astrocytes to Neurons. Cell Metab 23, 94-102.

Majmundar, A.J., Wong, W.J., and Simon, M.C. (2010). Hypoxia-inducible factors and the response to hypoxic stress. Mol Cell 40, 294-309.

Malmierca, M.S., Anderson, L.A., and Antunes, F.M. (2015). The cortical modulation of stimulusspecific adaptation in the auditory midbrain and thalamus: a potential neuronal correlate for predictive coding. Front Syst Neurosci 9, 19.

Manhães, A.C., Krahe, T.E., Caparelli-Dáquer, E., Ribeiro-Carvalho, A., Schmidt, S.L., and Filgueiras, C.C. (2003). Neonatal transection of the corpus callosum affects paw preference lateralization of adult Swiss mice. Neurosci Lett 348, 69-72.

Manhães, A.C., Schmidt, S.L., and Filgueiras, C.C. (2005). Callosal agenesis affects consistency of laterality in a paw preference task in BALB/CCF mice. Behav Brain Res 159, 43-49.

Markram, H., Toledo-Rodriguez, M., Wang, Y., Gupta, A., Silberberg, G., and Wu, C. (2004). Interneurons of the neocortical inhibitory system. Nat Rev Neurosci 5, 793-807.

McCasland, J.S., and Hibbard, L.S. (1997). GABAergic neurons in barrel cortex show strong, whiskerdependent metabolic activation during normal behavior. J Neurosci 17, 5509-5527.

McKenzie, I.A., Ohayon, D., Li, H., de Faria, J.P., Emery, B., Tohyama, K., and Richardson, W.D. (2014). Motor skill learning requires active central myelination. Science 346, 318-322.

McManus, I.C. (1992). Are paw preference differences in HI and LO mice the result of specific genes or of heterosis and fluctuating asymmetry? Behav Genet 22, 435-451.

Mellott, J.G., Bickford, M.E., and Schofield, B.R. (2014). Descending projections from auditory cortex to excitatory and inhibitory cells in the nucleus of the brachium of the inferior colliculus. Front Syst Neurosci 8, 188.

Mena, A., Ruiz-Salas, J.C., Puentes, A., Dorado, I., Ruiz-Veguilla, M., and De la Casa, L.G. (2016). Reduced prepulse inhibition as a biomarker of schizophrenia. Front Behav Neurosci 10, 202.

Merzenich, M.M., Jenkins, W.M., Johnston, P., Schreiner, C., Miller, S.L., and Tallal, P. (1996). Temporal processing deficits of language-learning impaired children ameliorated by training. Science $271,77-81$.

Micheva, K.D., Wolman, D., Mensh, B.D., Pax, E., Buchanan, J., Smith, S.J., and Bock, D.D. (2016). A large fraction of neocortical myelin ensheathes axons of local inhibitory neurons. Elife 5.

Micu, I., Plemel, J.R., Lachance, C., Proft, J., Jansen, A.J., Cummins, K., van Minnen, J., and Stys, P.K. (2016). The molecular physiology of the axo-myelinic synapse. Exp Neurol 276, 41-50.

Micu, I., Plemel, J.R., Caprariello, A.V., Nave, K.-A., and Stys, P.K. (2018). Axo-myelinic neurotransmission: a novel mode of cell signalling in the central nervous system. Nat Rev Neurosci 19, 49-58.

Miller, V.M., Gupta, D., Neu, N., Cotroneo, A., Boulay, C.B., and Seegal, R.F. (2013). Novel interhemispheric white matter connectivity in the BTBR mouse model of autism. Brain Res 1513, $26-33$. 
Minckler, D.S., McLean, I.W., and Tso, M.O. (1976). Distribution of axonal and glial elements in the rhesus optic nerve head studied by electron microscopy. Am J Ophthalmol 82, 179-187.

Misgeld, T., Kerschensteiner, M., Bareyre, F.M., Burgess, R.W., and Lichtman, J.W. (2007). Imaging axonal transport of mitochondria in vivo. Nat Methods 4, 559-561.

Mishra, R., Sanju, H.K., and Kumar, P. (2016). Auditory Temporal Resolution in Individuals with Diabetes Mellitus Type 2. International Archives of Otorhinolaryngology 20, 327-330.

Misko, A.L., Sasaki, Y., Tuck, E., Milbrandt, J., and Baloh, R.H. (2012). Mitofusin2 mutations disrupt axonal mitochondrial positioning and promote axon degeneration. J Neurosci 32, 4145-4155.

Miu, A.C., Heilman, R.M., Paşca, S.P., Stefan, C.A., Spânu, F., Vasiu, R., Olteanu, A.I., and Miclea, M. (2006). Behavioral effects of corpus callosum transection and environmental enrichment in adult rats. Behav Brain Res 172, 135-144.

Morell, P., and Quarles, R. (1999). Characteristic Composition of Myelin. In Basic Neurochemistry: Molecular, Cellular and Medical Aspects, G. Siegel, B. Agranoff, and R. Albers, eds. (LippincottRaven), .

Moreno-Paublete, R., Canlon, B., and Cederroth, C.R. (2017). Differential Neural Responses Underlying the Inhibition of the Startle Response by Pre-Pulses or Gaps in Mice. Front Cell Neurosci $11,19$.

Morgan, C.A., and Grillon, C. (1999). Abnormal mismatch negativity in women with sexual assaultrelated posttraumatic stress disorder. Biol Psychiatry 45, 827-832.

Morrison, B.M., Lee, Y., and Rothstein, J.D. (2013). Oligodendroglia: metabolic supporters of axons. Trends Cell Biol 23, 644-651.

Moser, T., and Starr, A. (2016). Auditory neuropathy--neural and synaptic mechanisms. Nat Rev Neurol 12, 135-149.

Muluk, N.B., Yalçinkaya, F., and Keith, R.W. (2011). Random gap detection test and random gap detection test-expanded: Results in children with previous language delay in early childhood. Auris Nasus Larynx 38, 6-13.

Musiek, F.E., Shinn, J.B., Jirsa, R., Bamiou, D.-E., Baran, J.A., and Zaida, E. (2005). GIN (Gaps-InNoise) test performance in subjects with confirmed central auditory nervous system involvement. Ear Hear 26, 608-618.

Näätänen, R., Tervaniemi, M., Sussman, E., Paavilainen, P., and Winkler, I. (2001). Primitive intelligence" in the auditory cortex. Trends Neurosci 24, 283-288.

Naito, R., Murofushi, T., Mizutani, M., and Kaga, K. (1999). Auditory brainstem responses, electrocochleograms, and cochlear microphonics in the myelin deficient mutant hamster "bt". Hear Res $136,44-48$.

Natan, R.G., Briguglio, J.J., Mwilambwe-Tshilobo, L., Jones, S.I., Aizenberg, M., Goldberg, E.M., and Geffen, M.N. (2015). Complementary control of sensory adaptation by two types of cortical interneurons. Elife 4.

Nave, K.-A. (2010). Myelination and support of axonal integrity by glia. Nature 468, 244-252. 
Nave, K.-A., and Ehrenreich, H. (2014). Myelination and oligodendrocyte functions in psychiatric diseases. JAMA Psychiatry 71, 582-584.

Nave, K.-A., and Trapp, B.D. (2008). Axon-glial signaling and the glial support of axon function. Annu Rev Neurosci 31, 535-561.

Neef, N.E., Sommer, M., Neef, A., Paulus, W., von Gudenberg, A.W., Jung, K., and Wüstenberg, T. (2012). Reduced speech perceptual acuity for stop consonants in individuals who stutter. J Speech Lang Hear Res 55, 276-289.

Nelken, I. (2014). Stimulus-specific adaptation and deviance detection in the auditory system: experiments and models. Biol Cybern 108, 655-663.

Nelson, A.D., and Jenkins, P.M. (2017). Axonal membranes and their domains: assembly and function of the axon initial segment and node of ranvier. Front Cell Neurosci 11, 136.

Nesse, W.H., Maler, L., and Longtin, A. (2010). Biophysical information representation in temporally correlated spike trains. Proc Natl Acad Sci U S A 107, 21973-21978.

Netrakanti, P.R., Cooper, B.H., Dere, E., Poggi, G., Winkler, D., Brose, N., and Ehrenreich, H. (2015). Fast cerebellar reflex circuitry requires synaptic vesicle priming by munc13-3. Cerebellum 14, 264-283.

Nguyen Trong, M., Bojak, I., and Knösche, T.R. (2013). Associating spontaneous with evoked activity in a neural mass model of visual cortex. Neuroimage $66,80-87$.

Noebels, J.L., Marcom, P.K., and Jalilian-Tehrani, M.H. (1991). Sodium channel density in hypomyelinated brain increased by myelin basic protein gene deletion. Nature 352, 431-434.

Nualart-Marti, A., Solsona, C., and Fields, R.D. (2013). Gap junction communication in myelinating glia. Biochim Biophys Acta 1828, 69-78.

O’Keefe, J., and Nadel, L. The' ' hippocampus as a cognitive map (Oxford: Clarendon Press).

Ocklenburg, S., Gerding, W.M., Arning, L., Genç, E., Epplen, J.T., Güntürkün, O., and Beste, C. (2016a). Myelin genes and the corpus callosum: proteolipid protein 1 (PLP1) and contactin 1 (CNTN1) gene variation modulates interhemispheric integration. Mol Neurobiol 1-9.

Ocklenburg, S., Friedrich, P., Güntürkün, O., and Genç, E. (2016b). Intrahemispheric white matter asymmetries: the missing link between brain structure and functional lateralization? Rev Neurosci 27 , 465-480.

Ocklenburg, S., Schmitz, J., Moinfar, Z., Moser, D., Klose, R., Lor, S., Kunz, G., Tegenthoff, M., Faustmann, P., Francks, C., et al. (2017). Epigenetic regulation of lateralized fetal spinal gene expression underlies hemispheric asymmetries. Elife 6.

Ocklenburg, S., Gerding, W.M., Raane, M., Arning, L., Genç, E., Epplen, J.T., Güntürkün, O., and Beste, C. (2018). PLP1 gene variation modulates leftward and rightward functional hemispheric asymmetries. Mol Neurobiol.

Ohno, N., Kidd, G.J., Mahad, D., Kiryu-Seo, S., Avishai, A., Komuro, H., and Trapp, B.D. (2011). Myelination and axonal electrical activity modulate the distribution and motility of mitochondria at CNS nodes of Ranvier. J Neurosci 31, 7249-7258. 
Oluich, L.-J., Stratton, J.A.S., Xing, Y.L., Ng, S.W., Cate, H.S., Sah, P., Windels, F., Kilpatrick, T.J., and Merson, T.D. (2012). Targeted ablation of oligodendrocytes induces axonal pathology independent of overt demyelination. J Neurosci 32, 8317-8330.

Osorio, N., Cathala, L., Meisler, M.H., Crest, M., Magistretti, J., and Delmas, P. (2010). Persistent Nav1.6 current at axon initial segments tunes spike timing of cerebellar granule cells. J Physiol (Lond) $588,651-670$.

Pacey, L.K.K., Xuan, I.C.Y., Guan, S., Sussman, D., Henkelman, R.M., Chen, Y., Thomsen, C., and Hampson, D.R. (2013). Delayed myelination in a mouse model of fragile $X$ syndrome. Hum Mol Genet 22, 3920-3930.

Pajevic, S., Basser, P.J., and Fields, R.D. (2014). Role of myelin plasticity in oscillations and synchrony of neuronal activity. Neuroscience 276, 135-147.

Parham, K., Bonaiuto, G., Carlson, S., Turner, J.G., D’Angelo, W.R., Bross, L.S., Fox, A., Willott, J.F., and Kim, D.O. (2000). Purkinje cell degeneration and control mice: responses of single units in the dorsal cochlear nucleus and the acoustic startle response. Hear Res 148, 137-152.

Parikh, S., Bernard, G., Leventer, R.J., van der Knaap, M.S., van Hove, J., Pizzino, A., McNeill, N.H., Helman, G., Simons, C., Schmidt, J.L., et al. (2015). A clinical approach to the diagnosis of patients with leukodystrophies and genetic leukoencephelopathies. Mol Genet Metab 114, 501-515.

Pérez-Escuredo, J., Van Hée, V.F., Sboarina, M., Falces, J., Payen, V.L., Pellerin, L., and Sonveaux, P. (2016). Monocarboxylate transporters in the brain and in cancer. Biochim Biophys Acta 1863, 24812497.

Pérez-González, D., and Malmierca, M.S. (2014). Adaptation in the auditory system: an overview. Front Integr Neurosci 8, 19.

Peters, A. (2009). The effects of normal aging on myelinated nerve fibers in monkey central nervous system. Front Neuroanat 3, 11.

Peters, A., and Sethares, C. (2003). Is there remyelination during aging of the primate central nervous system? J Comp Neurol 460, 238-254.

Peyvandi, A., Naghibzadeh, B., and Ahmady Roozbahany, N. (2010). Neuro-otologic manifestations of multiple sclerosis. Arch Iran Med 13, 188-192.

Phillips, D.P. (1999). Auditory gap detection, perceptual channels, and temporal resolution in speech perception. J Am Acad Audiol 10, 343-354.

Poggi, G., Boretius, S., Möbius, W., Moschny, N., Baudewig, J., Ruhwedel, T., Hassouna, I., Wieser, G.L., Werner, H.B., Goebbels, S., et al. (2016). Cortical network dysfunction caused by a subtle defect of myelination. Glia 64, 2025-2040.

Popeláŕ, J., Díaz Gómez, M., Lindovský, J., Rybalko, N., Burianová, J., Oohashi, T., and Syka, J. (2017). The absence of brain-specific link protein Bral2 in perineuronal nets hampers auditory temporal resolution and neural adaptation in mice. Physiol Res 66, 867-880. 
Popko, B., Puckett, C., Lai, E., Shine, H.D., Readhead, C., Takahashi, N., Hunt, S.W., Sidman, R.L., and Hood, L. (1987). Myelin deficient mice: expression of myelin basic protein and generation of mice with varying levels of myelin. Cell 48, 713-721.

Preslar, J., Kushner, H.I., Marino, L., and Pearce, B. (2014). Autism, lateralisation, and handedness: a review of the literature and meta-analysis. Laterality 19, 64-95.

Privat, A., Jacque, C., Bourre, J.M., Dupouey, P., and Baumann, N. (1979). Absence of the major dense line in myelin of the mutant mouse "shiverer". Neurosci Lett 12, 107-112.

Radziwon, K.E., June, K.M., Stolzberg, D.J., Xu-Friedman, M.A., Salvi, R.J., and Dent, M.L. (2009). Behaviorally measured audiograms and gap detection thresholds in CBA/CaJ mice. J Comp Physiol A Neuroethol Sens Neural Behav Physiol 195, 961-969.

Ramos, A., and Mormède, P. (1998). Stress and emotionality: a multidimensional and genetic approach. Neurosci Biobehav Rev 22, 33-57.

Rappaport, J., Gulliver, J., Phillips, D., Van Dorpe, R., Maxner, C., and Bhan, V. (1994). Auditory temporal resolution in multiple sclerosis. J Otolaryngol.

Rasband, M.N. (2010). The axon initial segment and the maintenance of neuronal polarity. Nat Rev Neurosci 11, 552-562.

Rasband, M.N. (2011). Composition, assembly, and maintenance of excitable membrane domains in myelinated axons. Semin Cell Dev Biol 22, 178-184.

Rasband, M.N., and Trimmer, J.S. (2001). Developmental clustering of ion channels at and near the node of Ranvier. Dev Biol 236, 5-16.

Rasband, M.N., Trimmer, J.S., Peles, E., Levinson, S.R., and Shrager, P. (1999a). K+ channel distribution and clustering in developing and hypomyelinated axons of the optic nerve. J Neurocytol 28, 319-331.

Rasband, M.N., Peles, E., Trimmer, J.S., Levinson, S.R., Lux, S.E., and Shrager, P. (1999b). Dependence of nodal sodium channel clustering on paranodal axoglial contact in the developing CNS. J Neurosci 19, 7516-7528.

Reynolds, E.S. (1963). The use of lead citrate at high $\mathrm{pH}$ as an electron-opaque stain in electron microscopy. J Cell Biol 17, 208-212.

Ribeiro, A.S., Eales, B.A., Lloyd-Price, J., and Biddle, F.G. (2014). Predictability and randomness of paw choices are critical elements in the behavioural plasticity of mouse paw preference. Animal Behaviour 98, 167-176.

Ribeiro-Carvalho, A., Abreu-Villaça, Y., Paes-Branco, D., Filgueiras, C.C., and Manhães, A.C. (2010). Novelty affects paw preference performance in adult mice. Animal Behaviour 80, 51-57.

Richardson, K.C., Jarett, L., and Finke, E.H. (1960). Embedding in epoxy resins for ultrathin sectioning in electron microscopy. Stain Technol 35, 313-323.

Richardson, W.D., Young, K.M., Tripathi, R.B., and McKenzie, I. (2011). NG2-glia as multipotent neural stem cells: fact or fantasy? Neuron 70, 661-673. 
Roach, A., Boylan, K., Horvath, S., Prusiner, S.B., and Hood, L.E. (1983). Characterization of cloned cDNA representing rat myelin basic protein: absence of expression in brain of shiverer mutant mice. Cell 34, 799-806.

Roach, A., Takahashi, N., Pravtcheva, D., Ruddle, F., and Hood, L. (1985). Chromosomal mapping of mouse myelin basic protein gene and structure and transcription of the partially deleted gene in shiverer mutant mice. Cell 42, 149-155.

Rogers, L.J. (2009). Hand and paw preferences in relation to the lateralized brain. Philos Trans R Soc Lond, B, Biol Sci 364, 943-954.

Rogers, L.J., and Andrew, R.J. (2002). Comparative vertebrate lateralization (Cambridge: Cambridge University Press).

Roncagliolo, M., Benítez, J., and Eguibar, J.R. (2000). Progressive deterioration of central components of auditory brainstem responses during postnatal development of the myelin mutant taiep rat. Audiol Neurootol 5, 267-275.

Rosen, S. (1992). Temporal information in speech: acoustic, auditory and linguistic aspects. Philos Trans R Soc Lond, B, Biol Sci 336, 367-373.

Rosenbluth, J. (1980). Central myelin in the mouse mutant shiverer. J Comp Neurol 194, 639-648.

Ross, B., Schneider, B., Snyder, J.S., and Alain, C. (2010). Biological markers of auditory gap detection in young, middle-aged, and older adults. PLoS ONE 5, e10101.

Rudnicki, M., Schoppe, O., Isik, M., Völk, F., and Hemmert, W. (2015). Modeling auditory coding: from sound to spikes. Cell Tissue Res 361, 159-175.

Rush, A.M., Dib-Hajj, S.D., and Waxman, S.G. (2005). Electrophysiological properties of two axonal sodium channels, Nav1.2 and Nav1.6, expressed in mouse spinal sensory neurones. J Physiol (Lond) $564,803-815$.

Ryan, H.E., Poloni, M., McNulty, W., Elson, D., Gassmann, M., Arbeit, J.M., and Johnson, R.S. (2000). Hypoxia-inducible factor-1alpha is a positive factor in solid tumor growth. Cancer Res 60, 4010-4015.

Saab, A.S., Tzvetavona, I.D., Trevisiol, A., Baltan, S., Dibaj, P., Kusch, K., Möbius, W., Goetze, B., Jahn, H.M., Huang, W., et al. (2016). Oligodendroglial NMDA receptors regulate glucose import and axonal energy metabolism. Neuron 91, 119-132.

Sachdev, P.S., Zhuang, L., Braidy, N., and Wen, W. (2013). Is Alzheimer's a disease of the white matter? Curr Opin Psychiatry 26, 244-251.

Sakata, S. (2016). State-dependent and cell type-specific temporal processing in auditory thalamocortical circuit. Sci Rep 6, 18873.

Salami, M., Itami, C., Tsumoto, T., and Kimura, F. (2003). Change of conduction velocity by regional myelination yields constant latency irrespective of distance between thalamus and cortex. Proc Natl Acad Sci U S A 100, 6174-6179.

Sampaio-Baptista, C., Khrapitchev, A.A., Foxley, S., Schlagheck, T., Scholz, J., Jbabdi, S., DeLuca, G.C., Miller, K.L., Taylor, A., Thomas, N., et al. (2013). Motor skill learning induces changes in white matter microstructure and myelination. J Neurosci 33, 19499-19503. 
Sánchez, I., Hassinger, L., Paskevich, P.A., Shine, H.D., and Nixon, R.A. (1996). Oligodendroglia regulate the regional expansion of axon caliber and local accumulation of neurofilaments during development independently of myelin formation. J Neurosci $16,5095-5105$.

Santos, M.A.R., Munhoz, M.S.L., Peixoto, M.A.L., Haase, V.G., Rodrigues, J.L., and Resende, L.M. (2006). Mismatch negativity contribution in multiple sclerosis patients. Braz J Otorhinolaryngol 72, 800807.

Satz, P., and Green, M.F. (1999). Atypical handedness in schizophrenia: some methodological and theoretical issues. Schizophr Bull 25, 63-78.

Schaette, R., and McAlpine, D. (2011). Tinnitus with a normal audiogram: physiological evidence for hidden hearing loss and computational model. J Neurosci 31, 13452-13457.

Schlegel, A.A., Rudelson, J.J., and Tse, P.U. (2012). White matter structure changes as adults learn a second language. J Cogn Neurosci 24, 1664-1670.

Schnupp, J., Nelken, I., and King, A. (2012). Auditory neuroscience: Making sense of sound (Cambridge, Mass: MIT Press).

Schnupp, J.W.H., Garcia-Lazaro, J.A., and Lesica, N.A. (2015). Periodotopy in the gerbil inferior colliculus: local clustering rather than a gradient map. Front Neural Circuits 9, 37.

Scholes, C., Palmer, A.R., and Sumner, C.J. (2011). Forward suppression in the auditory cortex is frequency-specific. Eur J Neurosci 33, 1240-1251.

Schölvinck, M.L., Friston, K.J., and Rees, G. (2012). The influence of spontaneous activity on stimulus processing in primary visual cortex. Neuroimage 59, 2700-2708.

Scholz, J., Klein, M.C., Behrens, T.E.J., and Johansen-Berg, H. (2009). Training induces changes in white-matter architecture. Nat Neurosci 12, 1370-1371.

Schreiner, C.E., and Langner, G. (1988). Periodicity coding in the inferior colliculus of the cat. II. Topographical organization. J Neurophysiol 60, 1823-1840.

Schreiner, C.E., and Polley, D.B. (2014). Auditory map plasticity: diversity in causes and consequences. Curr Opin Neurobiol 24, 143-156.

Seidl, A.H. (2014). Regulation of conduction time along axons. Neuroscience 276, 126-134.

Seidl, A.H., Rubel, E.W., and Barría, A. (2014). Differential conduction velocity regulation in ipsilateral and contralateral collaterals innervating brainstem coincidence detector neurons. J Neurosci 34, 49144919.

Semenza, G.L. (2012). Hypoxia-inducible factors in physiology and medicine. Cell 148, 399-408.

Sergeyenko, Y., Lall, K., Liberman, M.C., and Kujawa, S.G. (2013). Age-related cochlear synaptopathy: an early-onset contributor to auditory functional decline. J Neurosci 33, 13686-13694.

Shah, S.N., and Salamy, A. (1980). Auditory-evoked far-field potentials in myelin deficient mutant quaking mice. Neuroscience 5, 2321-2323. 
Shah, M.M., Migliore, M., Valencia, I., Cooper, E.C., and Brown, D.A. (2008). Functional significance of axonal Kv7 channels in hippocampal pyramidal neurons. Proc Natl Acad Sci U S A 105, 7869-7874.

Shailer, M.J., and Moore, B.C. (1983). Gap detection as a function of frequency, bandwidth, and level. J Acoust Soc Am 74, 467-473.

Sheedlo, H.J., Siegel, G.J., Desmond, T.J., and Ernst, S.A. (1987). (Na+ + K+)adenosinetriphosphatase in the brain of Shiverer (Shi/Shi) mice. Neurochem Res 12, 1081-1086.

Shen, Y., and Richards, V.M. (2013). Temporal modulation transfer function for efficient assessment of auditory temporal resolution. J Acoust Soc Am 133, 1031-1042.

Sidman, R.L., Conover, C.S., and Carson, J.H. (1985). Shiverer gene maps near the distal end of chromosome 18 in the house mouse. Cytogenet Cell Genet 39, 241-245.

Signore, P., Nosten-Bertrand, M., Chaoui, M., Roubertoux, P.L., Marchaland, C., and Perez-Diaz, F. (1991). An assessment of handedness in mice. Physiol Behav 49, 701-704.

Sinclair, J.L., Fischl, M.J., Alexandrova, O., Heß, M., Grothe, B., Leibold, C., and Kopp-Scheinpflug, C. (2017). Sound-Evoked Activity Influences Myelination of Brainstem Axons in the Trapezoid Body. J Neurosci 37, 8239-8255.

Sinha, K., Karimi-Abdolrezaee, S., Velumian, A.A., and Fehlings, M.G. (2006). Functional changes in genetically dysmyelinated spinal cord axons of shiverer mice: role of juxtaparanodal Kv1 family $\mathrm{K}_{+}$ channels. J Neurophysiol 95, 1683-1695.

Smith, K.J. (1994). Conduction properties of central demyelinated and remyelinated axons, and their relation to symptom production in demyelinating disorders. Eye (Lond) 8 ( Pt 2), 224-237.

Smith, K.J., Blakemore, W.F., Murray, J.A., and Patterson, R.C. (1982). Internodal myelin volume and axon surface area. A relationship determining myelin thickness? J Neurol Sci 55, 231-246.

Snaidero, N., and Simons, M. (2017). The logistics of myelin biogenesis in the central nervous system. Glia 65, 1021-1031.

Snaidero, N., Möbius, W., Czopka, T., Hekking, L.H.P., Mathisen, C., Verkleij, D., Goebbels, S., Edgar, J., Merkler, D., Lyons, D.A., et al. (2014). Myelin membrane wrapping of CNS axons by PI(3,4,5)P3dependent polarized growth at the inner tongue. Cell 156, 277-290.

Sontheimer, H. (1994). Voltage-dependent ion channels in glial cells. Glia 11, 156-172.

Spencer, P.S., Sabri, M.I., Schaumburg, H.H., and Moore, C.L. (1979). Does a defect of energy metabolism in the nerve fiber underlie axonal degeneration in polyneuropathies? Ann Neurol 5, 501507.

Spitzer, E., White-Schwoch, T., Carr, K.W., Skoe, E., and Kraus, N. (2015). Continued maturation of the click-evoked auditory brainstem response in preschoolers. J Am Acad Audiol 26, 30-35.

Starr, A., Picton, T.W., Sininger, Y., Hood, L.J., and Berlin, C.I. (1996). Auditory neuropathy. Brain 119 (Pt 3), 741-753.

Stathopoulos, P., Alexopoulos, H., and Dalakas, M.C. (2015). Autoimmune antigenic targets at the node of Ranvier in demyelinating disorders. Nat Rev Neurol 11, 143-156. 
Stedehouder, J., and Kushner, S.A. (2017). Myelination of parvalbumin interneurons: a parsimonious locus of pathophysiological convergence in schizophrenia. Mol Psychiatry 22, 4-12.

Stedehouder, J., Couey, J.J., Brizee, D., Hosseini, B., Slotman, J.A., Dirven, C.M.F., Shpak, G., Houtsmuller, A.B., and Kushner, S.A. (2017). Fast-spiking Parvalbumin Interneurons are Frequently Myelinated in the Cerebral Cortex of Mice and Humans. Cereb Cortex 27, 5001-5013.

Stocker, A.M., and O'Leary, D.D.M. (2016). Emx1 is required for neocortical area patterning. PLoS ONE 11, e0149900.

Strenzke, N., Chakrabarti, R., Al-Moyed, H., Müller, A., Hoch, G., Pangrsic, T., Yamanbaeva, G., Lenz, C., Pan, K.-T., Auge, E., et al. (2016). Hair cell synaptic dysfunction, auditory fatigue and thermal sensitivity in otoferlin lle515Thr mutants. EMBO J 35, 2519-2535.

Stys, P.K. (2011). The axo-myelinic synapse. Trends Neurosci 34, 393-400.

Stys, P.K., Ransom, B.R., and Waxman, S.G. (1991). Compound action potential of nerve recorded by suction electrode: a theoretical and experimental analysis. Brain Res 546, 18-32.

Susuki, K., and Kuba, H. (2016). Activity-dependent regulation of excitable axonal domains. J Physiol Sci 66, 99-104.

Taaseh, N., Yaron, A., and Nelken, I. (2011). Stimulus-specific adaptation and deviance detection in the rat auditory cortex. PLoS ONE 6, e23369.

Takahashi, N., Sakurai, T., Davis, K.L., and Buxbaum, J.D. (2011). Linking oligodendrocyte and myelin dysfunction to neurocircuitry abnormalities in schizophrenia. Prog Neurobiol 93, 13-24.

Takeda, S., and Endo, A. (1993). Paw preference in mice: a reappraisal. Physiol Behav 53, 727-730.

Talwar, S.K., Musial, P.G., and Gerstein, G.L. (2001). Role of mammalian auditory cortex in the perception of elementary sound properties. J Neurophysiol 85, 2350-2358.

Ter-Mikaelian, M., Sanes, D.H., and Semple, M.N. (2007). Transformation of temporal properties between auditory midbrain and cortex in the awake Mongolian gerbil. J Neurosci 27, 6091-6102.

Thompson, R.F., and Spencer, W.A. (1966). Habituation: a model phenomenon for the study of neuronal substrates of behavior. Psychol Rev 73, 16-43.

Tomassy, G.S., Berger, D.R., Chen, H.-H., Kasthuri, N., Hayworth, K.J., Vercelli, A., Seung, H.S., Lichtman, J.W., and Arlotta, P. (2014). Distinct profiles of myelin distribution along single axons of pyramidal neurons in the neocortex. Science 344, 319-324.

Trevisiol, A., Saab, A.S., Winkler, U., Marx, G., Imamura, H., Möbius, W., Kusch, K., Nave, K.-A., and Hirrlinger, J. (2017). Monitoring ATP dynamics in electrically active white matter tracts. Elife 6.

Trullas, R., and Skolnick, P. (1993). Differences in fear motivated behaviors among inbred mouse strains. Psychopharmacology (Berl) 111, 323-331.

Turrigiano, G.G. (1999). Homeostatic plasticity in neuronal networks: the more things change, the more they stay the same. Trends Neurosci 22, 221-227. 
Ulanovsky, N., Las, L., and Nelken, I. (2003). Processing of low-probability sounds by cortical neurons. Nat Neurosci 6, 391-398.

Ulanovsky, N., Las, L., Farkas, D., and Nelken, I. (2004). Multiple time scales of adaptation in auditory cortex neurons. J Neurosci 24, 10440-10453.

Valadbeigi, A., Weisi, F., Rohbakhsh, N., Rezaei, M., Heidari, A., and Rasa, A.R. (2014). Central auditory processing and word discrimination in patients with multiple sclerosis. Eur Arch Otorhinolaryngol 271, 2891-2896.

Valadbeigi, A., Rouhbakhsh, N., Mohammadkhani, G., Karimi, L.J., and Jalaie, S. (2017). The capability of gap in noise detection in patients with multiple sclerosis. Auditory and Vestibular Research.

Vallortigara, G., Rogers, L.J., and Bisazza, A. (1999). Possible evolutionary origins of cognitive brain lateralization. Brain Res Brain Res Rev 30, 164-175.

Verhulst, S., Jagadeesh, A., Mauermann, M., and Ernst, F. (2016). Individual differences in auditory brainstem response wave characteristics: relations to different aspects of peripheral hearing loss. Trends in Hearing 20.

Verkhratsky, A., and Kirchhoff, F. (2007). NMDA Receptors in glia. Neuroscientist 13, 28-37.

Vidal, C.N., Nicolson, R., DeVito, T.J., Hayashi, K.M., Geaga, J.A., Drost, D.J., Williamson, P.C., Rajakumar, N., Sui, Y., Dutton, R.A., et al. (2006). Mapping corpus callosum deficits in autism: an index of aberrant cortical connectivity. Biol Psychiatry 60, 218-225.

Wake, H., Ortiz, F.C., Woo, D.H., Lee, P.R., Angulo, M.C., and Fields, R.D. (2015). Nonsynaptic junctions on myelinating glia promote preferential myelination of electrically active axons. Nat Commun 6,7844 .

Walton, J.P., Frisina, R.D., Ison, J.R., and O'Neill, W.E. (1997). Neural correlates of behavioral gap detection in the inferior colliculus of the young CBA mouse. J Comp Physiol A 181, 161-176.

Walton, J.P., Barsz, K., and Wilson, W.W. (2008). Sensorineural hearing loss and neural correlates of temporal acuity in the inferior colliculus of the C57BL/6 mouse. J Assoc Res Otolaryngol 9, 90-101.

Wan, G., and Corfas, G. (2017). Transient auditory nerve demyelination as a new mechanism for hidden hearing loss. Nat Commun 8, 14487.

Wang, H., Allen, M.L., Grigg, J.J., Noebels, J.L., and Tempel, B.L. (1995a). Hypomyelination alters K+ channel expression in mouse mutants shiverer and Trembler. Neuron 15, 1337-1347.

Wang, P.J., Young, C., Liu, H.M., Chang, Y.C., and Shen, Y.Z. (1995b). Neurophysiologic studies and $\mathrm{MRI}$ in Pelizaeus-Merzbacher disease: comparison of classic and connatal forms. Pediatr Neurol 12, 47-53.

Wang, X., Qi, Q., Huang, C., Chomiak, T., and Luo, F. (2016). Duration sensitivity of neurons in the primary auditory cortex of albino mouse. Hear Res 332, 160-169.

Wang, X., Liu, Y., Li, X., Zhang, Z., Yang, H., Zhang, Y., Williams, P.R., Alwahab, N.S.A., Kapur, K., $\mathrm{Yu}, \mathrm{B}$., et al. (2017). Deconstruction of Corticospinal Circuits for Goal-Directed Motor Skills. Cell 171, 440-455.e14. 
Van Wart, A., and Matthews, G. (2006a). Impaired firing and cell-specific compensation in neurons lacking nav1. 6 sodium channels. J Neurosci 26, 7172-7180.

Van Wart, A., and Matthews, G. (2006b). Expression of sodium channels Nav1.2 and Nav1.6 during postnatal development of the retina. Neurosci Lett 403, 315-317.

Waters, N.S., and Denenberg, V.H. (1994). Analysis of two measures of paw preference in a large population of inbred mice. Behav Brain Res 63, 195-204.

Waxman, S.G., and Brill, M.H. (1978). Conduction through demyelinated plaques in multiple sclerosis: computer simulations of facilitation by short internodes. J Neurol Neurosurg Psychiatr 41, 408-416.

Wehr, M., and Metherate, R. (2011). Synaptic integration in auditory cortex. In The Auditory Cortex, J.A. Winer, and C.E. Schreiner, eds. (Boston, MA: Springer US), pp. 235-249.

Wehr, M., and Zador, A.M. (2005). Synaptic mechanisms of forward suppression in rat auditory cortex. Neuron 47, 437-445.

Wei, H., Ma, Y., Liu, J., Ding, C., Hu, F., and Yu, L. (2016). Proteomic analysis of cortical brain tissue from the BTBR mouse model of autism: Evidence for changes in STOP and myelin-related proteins. Neuroscience 312, 26-34.

Weible, A.P., Moore, A.K., Liu, C., DeBlander, L., Wu, H., Kentros, C., and Wehr, M. (2014a). Perceptual gap detection is mediated by gap termination responses in auditory cortex. Curr Biol 24, 1447-1455.

Weible, A.P., Liu, C., Niell, C.M., and Wehr, M. (2014b). Auditory cortex is required for fear potentiation of gap detection. J Neurosci 34, 15437-15445.

Weinberger, N.M. (2012). Plasticity in the Primary Auditory Cortex, Not What You Think it is: Implications for Basic and Clinical Auditory Neuroscience. Otolaryngology (Sunnyvale, Calif.) Suppl 3.

Wender, R., Brown, A.M., Fern, R., Swanson, R.A., Farrell, K., and Ransom, B.R. (2000). Astrocytic glycogen influences axon function and survival during glucose deprivation in central white matter. $J$ Neurosci 20,6804-6810.

Werner, H.B., Krämer-Albers, E.-M., Strenzke, N., Saher, G., Tenzer, S., Ohno-Iwashita, Y., De Monasterio-Schrader, P., Möbius, W., Moser, T., Griffiths, I.R., et al. (2013). A critical role for the cholesterol-associated proteolipids PLP and M6B in myelination of the central nervous system. Glia 61, 567-586.

Westenbroek, R.E., Noebels, J.L., and Catterall, W.A. (1992). Elevated Expression of Type II Na + Channels Axons of shiverer Mouse Brain in Hypomyelinated Axons of shiverer Mouse Brain. $J$ Neurosci.

Willott, J.F. (2006). Measurement of the auditory brainstem response (ABR) to study auditory sensitivity in mice. Curr Protoc Neurosci Chapter 8, Unit8.21B.

Windrem, M.S., Schanz, S.J., Guo, M., Tian, G.-F., Washco, V., Stanwood, N., Rasband, M., Roy, N.S., Nedergaard, M., Havton, L.A., et al. (2008). Neonatal chimerization with human glial progenitor cells can both remyelinate and rescue the otherwise lethally hypomyelinated shiverer mouse. Cell Stem Cell 2, 553-565. 
Xing, Y., Samuvel, D.J., Stevens, S.M., Dubno, J.R., Schulte, B.A., and Lang, H. (2012). Age-related changes of myelin basic protein in mouse and human auditory nerve. PLoS ONE 7, e34500.

$\mathrm{Xu}, \mathrm{H}$., and Li, X.-M. (2011). White matter abnormalities and animal models examining a putative role of altered white matter in schizophrenia. Schizophr Res Treatment 2011, 826976.

Yamada, R., and Kuba, H. (2016). Structural and functional plasticity at the axon initial segment. Front Cell Neurosci 10, 250.

Yamazaki, Y., Hozumi, Y., Kaneko, K., Sugihara, T., Fujii, S., Goto, K., and Kato, H. (2007). Modulatory effects of oligodendrocytes on the conduction velocity of action potentials along axons in the alveus of the rat hippocampal CA1 region. Neuron Glia Biol 3, 325-334.

Yeomans, J.S., and Frankland, P.W. (1995). The acoustic startle reflex: neurons and connections. Brain Res Brain Res Rev 21, 301-314.

Yuen, T.J., Silbereis, J.C., Griveau, A., Chang, S.M., Daneman, R., Fancy, S.P.J., Zahed, H., Maltepe, E., and Rowitch, D.H. (2014). Oligodendrocyte-encoded HIF function couples postnatal myelination and white matter angiogenesis. Cell 158, 383-396.

Zatorre, R.J., Fields, R.D., and Johansen-Berg, H. (2012). Plasticity in gray and white: neuroimaging changes in brain structure during learning. Nat Neurosci 15, 528-536.

Zeng, F.-G., Kong, Y.-Y., Michalewski, H.J., and Starr, A. (2005). Perceptual consequences of disrupted auditory nerve activity. J Neurophysiol 93, 3050-3063.

Zhong, L., and Mostoslavsky, R. (2010). SIRT6: a master epigenetic gatekeeper of glucose metabolism. Transcription 1, 17-21. 


\section{Acknowledgements}

First of all I would like to thank Livia de Hoz for all her support over the years in the direct supervision of this project. Not only at the professional level, but also personally it has been a pleasure working with her; to Klaus-Armin Nave for the constant support and the possibility of working in such a great environment. Livia and Klaus: I am very grateful you both embarked on the unknown, and we all learned to sail together.

To my Thesis Advisory Committee Members: Mikael Simons and Swen Hülsmann, thank you for the interesting discussions and the support towards this project; to Wiebke Möbius, Iva Tzvetanova and Kathrin Kusch for sharing the mouse lines that made this project possible and for all the interesting discussions and input to the project; Iva also for the help with the immunostainings; Torben Ruhwedel and Martin Meschkat for the help with EM analysis, molecular characterizations of the models, and maintenance of the mice, and for always being very critical, open and supportive; to Andrea Trevisiol for the help with optic nerve recordings and the interesting discussions; to Gudrun Fricke-Bode and Cornelia Casper for the technical support with genotyping and care of $\mathrm{MBP}^{\text {shi }}$ mice; To Arne Battefeld for the AIS analysis, and for being very supportive and critical.

To my lab-mates Chi Chen, Swati Subramanian, Philipp van Kronenberg, Linus Milinski, Ebrahim Asadollahi and the adopted Alejandro Restrepo, thanks for making the lab a great place, for being so supportive and helpful, for the musical recommendations and for the amazing international cooking times.

To Michael Hörner, Sandra Drube, the International Max Planck Research School and the Georg-August-Universität Göttingen, for making possible this great international PhD experience. To the DAAD and CONACyT for funding my participation in this project.

To all my friends far away and to my new family in Göttingen, thank you for being always there and making this experience so special. I specially want to thank Diana Corona and Armando Moore for the unconditional love, for being always there for me, and for the endurance of all these years being apart; to Mami because I know you would have been the proudest person for the completion of this work; Diego Giraldo, thank you for everything, I could not have done this without you. 


\section{Appendix}

\subsection{List of abbreviations}

\begin{tabular}{|c|c|}
\hline $\mathrm{ACx}$ & auditory cortex \\
\hline ABR & auditory brainstem response \\
\hline AIS & axon initial segment \\
\hline AMS & axo-myelinic synapse \\
\hline ANF & auditory nerve fibers \\
\hline ANP & auditory neuropathies \\
\hline ASR & acoustic startle response \\
\hline AP & action potential \\
\hline BBN & broad band noise \\
\hline BTS & brain/tissue slice \\
\hline CAP & compound action potential \\
\hline CC & corpus callosum \\
\hline $\mathrm{CN}$ & cochlear nucleus \\
\hline CNS & central nervous system \\
\hline CV & conduction velocity \\
\hline dB & decibels \\
\hline EM & electron microscopy \\
\hline GD & glucose deprivation \\
\hline HRS & high-rate stimulation \\
\hline $\mathrm{Hz}$ & hertz \\
\hline IC & inferior colliculus \\
\hline $\mathrm{ICI}$ & inter-click interval \\
\hline ITI & inter-trial interval \\
\hline LPE & left paw entries \\
\hline MBP & myelin basic protein \\
\hline MGB & medial geniculate body \\
\hline MUA & multiunit activity \\
\hline OLs & oligodendrocytes \\
\hline
\end{tabular}


Appendix

\begin{tabular}{|c|c|}
\hline ON & optic nerve \\
\hline PLP & Proteolipid protein \\
\hline PMD & Palizaeus-Merzbacher disease \\
\hline PSTH & post-stimulus time histogram \\
\hline PPE & preferred paw entries \\
\hline PPI & pre-pulse inhibition \\
\hline $\mathbf{P V}^{+}$ & Parvoalbumin positive cells \\
\hline RPE & right paw entries \\
\hline RMS & root mean square \\
\hline SC & Schwann cell \\
\hline SD & standard deviation \\
\hline SEM & standard error of the mean \\
\hline SPL & sound pressure level \\
\hline TPE & total paw entries \\
\hline TTX & tetradotoxin \\
\hline WT & wild type \\
\hline
\end{tabular}




\subsection{Additional figures}

A

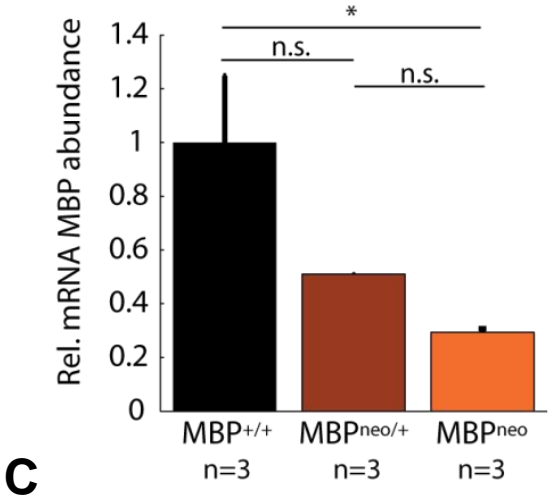

B

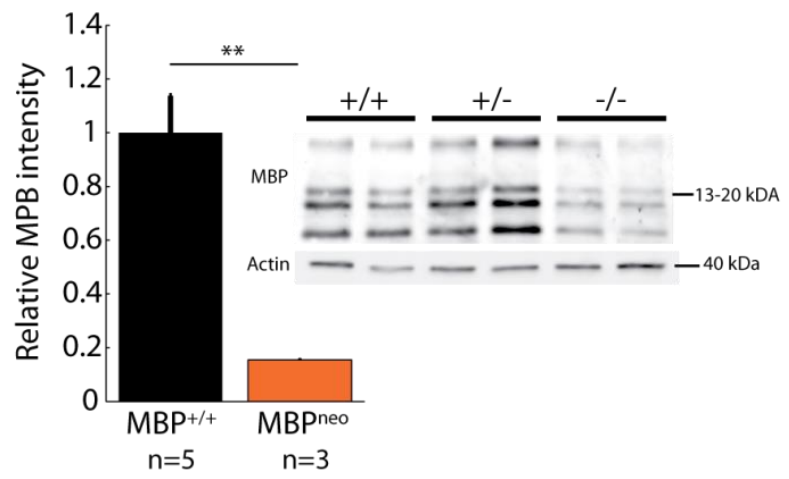

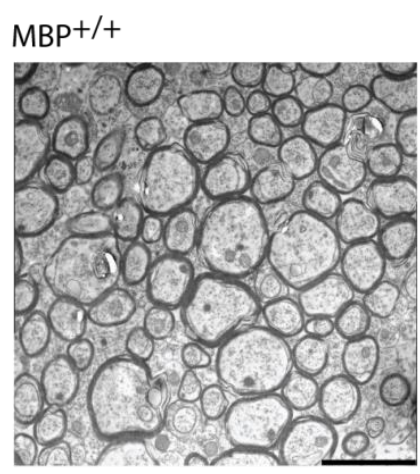

D

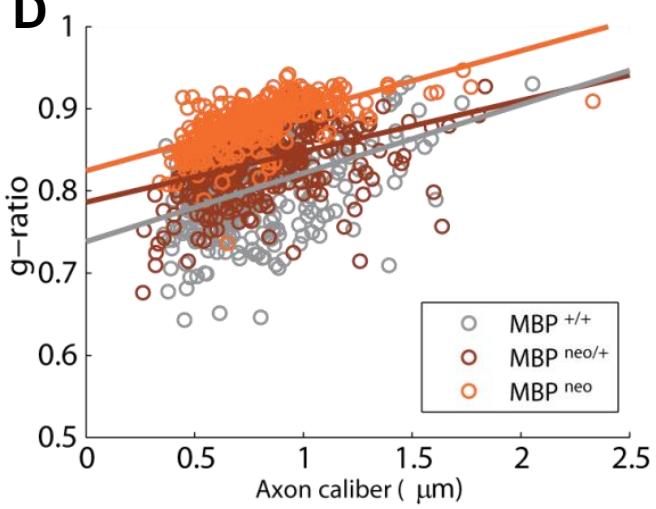

MBPneo/+

MBPneo
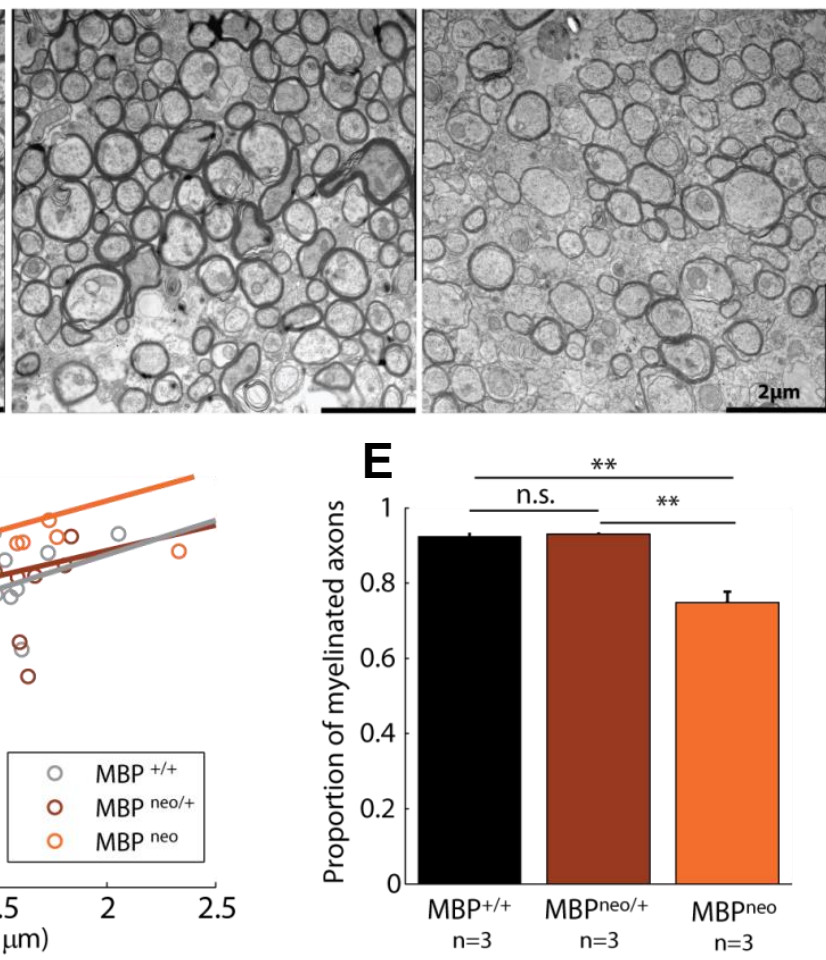

Figure 49. Molecular characterization of $\mathrm{MBP}^{\text {neo }}$ mice $\mathrm{MBP}^{\text {neo }}$ mice (orange) had a strong dysmyelination phenotype but not to the extent of $\mathrm{MBP}^{\text {shi }}$ mice. They showed a significant reduction in MBP expression both at the A) mRNA (70\%) and B) protein $(80 \%)$ levels $(p=0.047$, and $p=0.0037$ respectively). C) Mutant (right) mice showed compact myelin, but it was significantly thinner than control (left) animals, as quantified by the D) g-ratio $(p=0.024)$ of axons measured from the caudal corpus callosum. E) In addition, in the optic nerve, MBP ${ }^{\text {neo }}$ mice have a significant decrease of the number of axons that are myelinated $(p=0.004)$. These quantifications were performed in animals that were around 8 weeks of age, similar to the age at which auditory experiments were performed. $\mathrm{MBP}^{\text {neo/t }}$ showed an intermediate phenotype. Images and data of quantifications kindly provided by Martin Meschkat. 


$$
\begin{aligned}
& \text { - MBPshiv } \\
& \text { - MBPshiv + 4-AP(100 } \mu \mathrm{M}) \\
& \text {...' MBPshiv }+4-\mathrm{AP}(100 \mu \mathrm{M}) \text { washout }
\end{aligned}
$$
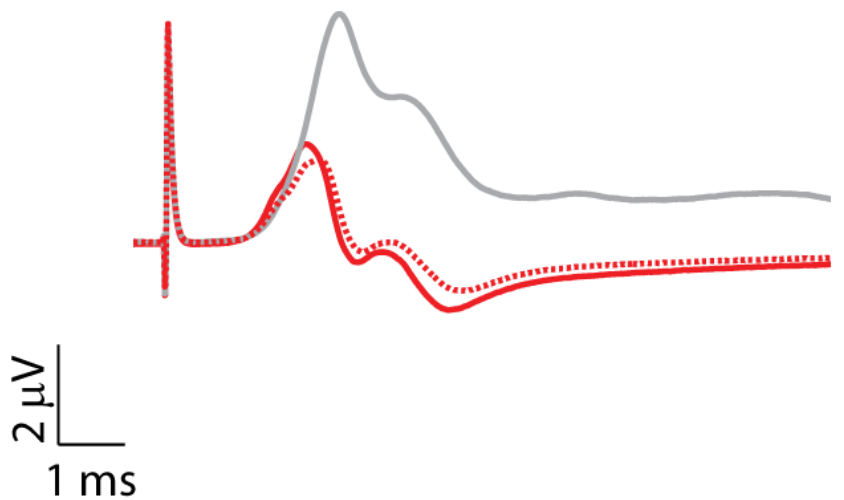

Figure 50. Example of the effect of 4-AP in MBP ${ }^{s h t}$ optic nerve CAP.

4-AP is a selective $K_{v} 1$ channel family blocker. When applied to $\mathrm{MBP}^{\text {shi }}$ optic nerves (solid red line), it changes the shape of the CAP, reducing the hyperpolarizing phase and increasing strikingly the amplitude of the CAP (gray line). Its action is reversible as it can be appreciated in the dotted red line corresponding to the washout.

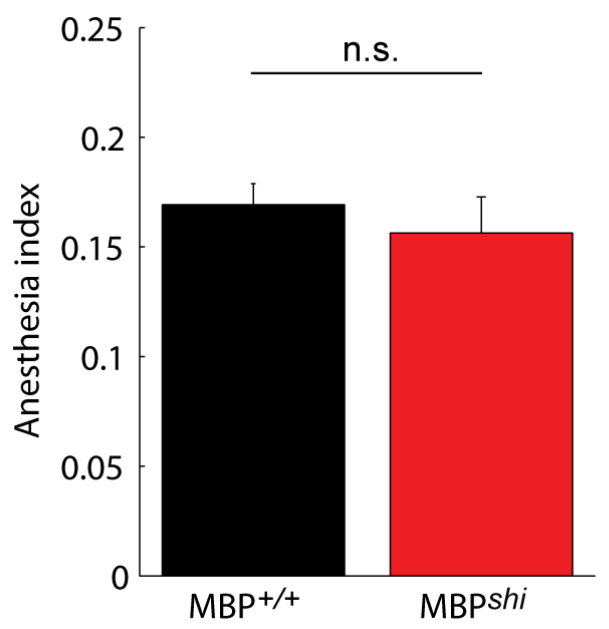

Figure 51. Anesthesia levels of $\mathrm{MBP}^{s h t}$ and control animals for acute recordings in the auditory cortex.

The level of anesthesia was calculated with the amount of total anesthetic received divided by the duration of the recording session. No differences are seen between control (black) and MBP shi mice ( $\mathrm{p}=0.77)$. 


\subsection{Breakdown of collaborations}

\begin{tabular}{|c|c|c|c|c|}
\hline Collaboration & $\begin{array}{c}\text { Personal } \\
\text { contribution }\end{array}$ & $\begin{array}{c}\text { External } \\
\text { contribution }\end{array}$ & Collaborator(s) & Affiliation \\
\hline $\begin{array}{l}\text { Electron } \\
\text { microscopy }\end{array}$ & $\begin{array}{l}\text { Sample slicing, } \\
\text { area perforation } \\
\text { and } \\
\text { quantification, } \\
\text { figure making }\end{array}$ & $\begin{array}{l}\text { Perfusion, sample } \\
\text { preparation, } \\
\text { sample processing } \\
\text { for EM, imaging }\end{array}$ & $\begin{array}{l}\text {-Torben Ruhwedel } \\
\text { - Wiebke Möbius }\end{array}$ & $\begin{array}{l}\text { Max Planck Institute } \\
\text { of Experimental } \\
\text { Medicine }\end{array}$ \\
\hline $\begin{array}{c}\text { MBP }^{\text {neo }} \\
\text { characterization }\end{array}$ & $\begin{array}{l}\text { Final data } \\
\text { analysis and } \\
\text { figure making }\end{array}$ & $\begin{array}{l}\text { EM sample } \\
\text { preparation, } \\
\text { imaging, g-ratio } \\
\text { and axon caliber } \\
\text { quantification, } \\
\text { western blotting, } \\
\text { mRNA } \\
\text { quantification }\end{array}$ & $\begin{array}{l}\text {-Martin Meschkat } \\
\text { - Wiebke Möbius }\end{array}$ & $\begin{array}{l}\text { Max Planck Institute } \\
\text { of Experimental } \\
\text { Medicine }\end{array}$ \\
\hline ABR amplifier & $\begin{array}{l}\text { Setup, } \\
\text { calibration, } \\
\text { experiments, } \\
\text { analysis }\end{array}$ & $\begin{array}{l}\text { ABR amplifier } \\
\text { engineering and } \\
\text { setup } \\
\text { troubleshooting }\end{array}$ & -Gerhard Hoch & $\begin{array}{l}\text { University Medical } \\
\text { Center Göttingen }\end{array}$ \\
\hline $\begin{array}{c}\text { ABR } \\
\text { measurements } \\
\text { for } \text { MBP }^{\text {neo }} \text { mice }\end{array}$ & $\begin{array}{l}\text { Data analysis } \\
\text { and figure } \\
\text { making }\end{array}$ & $\begin{array}{l}\text { ABR procedure, } \\
\text { data processing }\end{array}$ & $\begin{array}{l}\text {-Nadine Herrmann } \\
\text {-Nicola Strenzke }\end{array}$ & $\begin{array}{l}\text { University Medical } \\
\text { Center Göttingen }\end{array}$ \\
\hline $\begin{array}{l}\text { Optic nerve } \\
\text { recordings in } \\
\text { MBPshi }\end{array}$ & $\begin{array}{l}\text { Solutions } \\
\text { preparation, } \\
\text { running of } \\
\text { experiments, } \\
\text { data processing, } \\
\text { data analysis, } \\
\text { figure making. }\end{array}$ & $\begin{array}{l}\text { Optic nerve } \\
\text { preparation and } \\
\text { mounting, software } \\
\text { troubleshooting }\end{array}$ & - Andrea Trevisiol & $\begin{array}{l}\text { Max Planck Institute } \\
\text { of Experimental } \\
\text { Medicine }\end{array}$ \\
\hline $\begin{array}{l}\text { Optic nerve } \\
\text { recordings in } \\
\text { MBP }^{\text {neo }}\end{array}$ & $\begin{array}{l}\text { Solutions } \\
\text { preparation, data } \\
\text { processing, data } \\
\text { analysis, figure } \\
\text { making. }\end{array}$ & $\begin{array}{l}\text { Optic nerve } \\
\text { preparation, } \\
\text { running of } \\
\text { experiments }\end{array}$ & & \\
\hline \multirow[t]{2}{*}{$\begin{array}{c}\text { AIS } \\
\text { characterization }\end{array}$} & \multirow[t]{2}{*}{$\begin{array}{l}\text { Final analysis } \\
\text { and figure } \\
\text { making }\end{array}$} & $\begin{array}{l}\text {-Sample } \\
\text { preparation, } \\
\text { immunostainings, } \\
\text { imaging, }\end{array}$ & $\begin{array}{l}\text {-lva Tzvetanova } \\
\text {-Annette Fahrenholz }\end{array}$ & $\begin{array}{l}\text { Max Planck Institute } \\
\text { of Experimental } \\
\text { Medicine }\end{array}$ \\
\hline & & -Analysis & $\begin{array}{l}\text {-Arne Battefeld } \\
\text {-Maarten Kole }\end{array}$ & $\begin{array}{l}\text { Netherlands Institute } \\
\text { for Neuroscience }\end{array}$ \\
\hline
\end{tabular}




\section{List of Figures}

Figure 1. Schematic overview of the auditory pathway and the recorded areas..................... 15

Figure 2. Scheme of the MUA detection using a thresholding method..................................... 17

Figure 3. Scheme of the sound protocols used for acute auditory cortex recordings............... 19

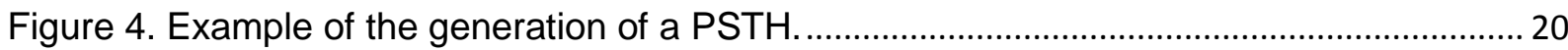

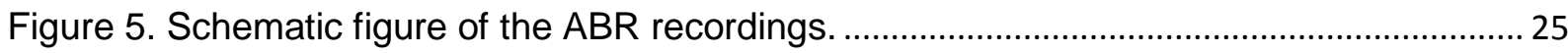

Figure 6. Schematic of the optic nerve recording setup......................................................... 28

Figure 7. Compound action potential properties.................................................................. 29

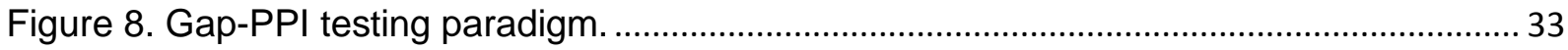

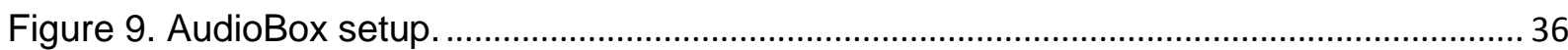

Figure 10. Summary of the AudioBox training protocol for gap-detection................................ 37

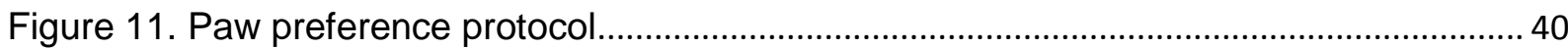

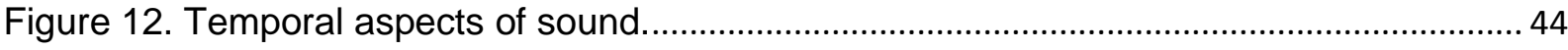

Figure 13. Examples of rate coding and spiking synchrony in different auditory stations. ...... 46

Figure 14. Example of forward suppression in the ACx..................................................... 46

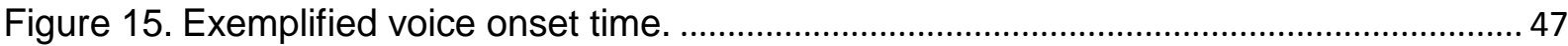

Figure 16. Myelin profiles in the inferior colliculus and the auditory cortex of MBP ${ }^{\text {shi }}$ mice..... 52

Figure 17. Subcortical auditory processing is impaired in $\mathrm{MBP}^{\text {shi }}$ mice. ................................... 55

Figure 18. Auditory cortex and inferior colliculus responses are impaired in MBP ${ }^{\text {shi }}$ mice. ..... 56

Figure 19. Frequency tuning is normal in $\mathrm{MBP}^{\text {shi }}$ mice............................................................ 57

Figure 20. Dysmyelination generates a reduction in the length of the axon initial segment.... 59

Figure 21. White matter abnormalities arise from the complete loss of MBP ......................... 61

Figure 22. $\mathrm{MBP}^{\text {shi }}$ mice have impaired temporal acuity in the auditory cortex..........................64 64

Figure 23. Temporal reliability is affected in $\mathrm{MBP}^{\text {shi }}$ mice. ........................................................6 66

Figure 24. Deviance detection is potentiated in $\mathrm{MBP}^{\text {shi }}$ mice. ...................................................68

Figure 25. Axons from the white matter of the $\mathrm{MBP}^{\text {shi }}$ mice show a strong decay when

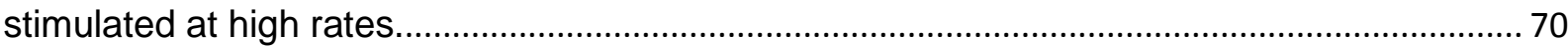

Figure 26. 4-AP applied to $\mathrm{MBP}^{\text {shi }}$ optic nerve, rescues amplitude but not latency changes or

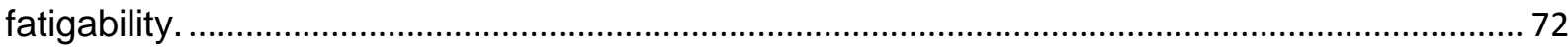

Figure 27. Subcortical auditory processing is not impaired $\mathrm{MBP}^{\mathrm{emx}}$ mice but a strong reduction

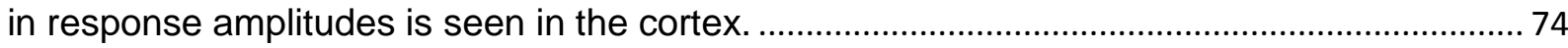

Figure 28. Impaired temporal acuity in $\mathrm{MBP}^{\mathrm{emx}}$ mice................................................................ 76

Figure 29. Rate coding is affected in the auditory cortex of $\mathrm{MBP}^{\mathrm{emx}}$ mice................................. 78

Figure 30. Subcortical auditory processing is impaired $\mathrm{MBP}^{\text {neo }}$ mice........................................ 80

Figure 31. Auditory cortex and inferior colliculus responses are impaired in $\mathrm{MBP}^{\text {neo }}$ mice. .... 81

Figure 32. White matter abnormalities that arise from $\mathrm{MBP}^{\text {neo }}$ mutation. ................................ 84

Figure 33. Impaired temporal acuity in $\mathrm{MBP}^{\text {neo }}$ mice. ............................................................... 86

Figure 34. Rate coding is slightly affected in the auditory cortex of $\mathrm{MBP}^{\text {neo }}$ mice. ................... 88

Figure 35. A paradigm of pre-pulse inhibition of the acoustic startle reflex using gaps reveals that $\mathrm{MBP}^{\text {neo }}$ mice have a behavioral impairment of temporal acuity...................................... 91

Figure 36. Behavioral gap-detection in the AudioBox is impaired in $\mathrm{MBP}^{\text {neo }}$ mice. .................. 94 
Figure 37. Schematic representation of the metabolic support from oligodendrocytes to axons using lactate.

Figure 38. Subcortical auditory processing is not impaired in $\mathrm{MCT} 1^{+/}$mice.

Figure 39. Auditory cortex responses in $\mathrm{MCT}^{+/ /}$and $\mathrm{Hif} 1 \alpha^{\mathrm{fl} / \mathrm{fl} /} ; \mathrm{PLP}-\mathrm{CreERT} 2^{+}$mice. 118

Figure 40. Temporal acuity is not impaired in the auditory cortex of $\mathrm{MCT} 1^{+/-}$mice. 120

Figure 41. Temporal acuity is impaired in the auditory cortex of Hif $1 \mathrm{a}^{f / f} \mathrm{f} ; \mathrm{PLP}-\mathrm{creRT}^{+}{ }^{+}$mice. 122 Figure 42. Rate coding is slightly affected in the auditory cortex of $\mathrm{MCT} 1^{+/}$mice. 124

Figure 43. Rate coding is affected in the auditory cortex of Hif $1 \alpha^{f f / f l}$;PLP-creERT2 ${ }^{+}$mice..... 126

Figure 44. The diverse roles of oligodendrocytes for the maintenance of axonal stability. ... 132

Figure 45. Patterns of behavioral laterality can be found in most vertebrates. 134

Figure 46. Behavioral laterality is impaired in $\mathrm{MBP}^{\text {shi }}$ mice. 137

Figure 47. Behavioral laterality is not impaired in $\mathrm{MBP}^{\mathrm{emx}}$ mice. 139

Figure 48. Behavioral laterality is not impaired in $\mathrm{MBP}^{\mathrm{fl} / f /} ; \mathrm{PLP}^{-c r e E R T 2}{ }^{+}$mice. ....................... 140

Figure 49. Molecular characterization of $\mathrm{MBP}^{\text {neo }}$ mice.

Figure 50. Example of the effect of 4-AP in MBPshi optic nerve CAP 174

Figure 51. Anesthesia levels of $\mathrm{MBP}^{\text {shi }}$ and control animals for acute recordings in the auditory cortex. 174 


\section{List of Tables}

Table 1. Summary of numbers and characteristics of the experimental animals used for acute

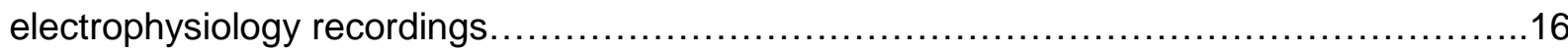

Table 2. Summary of experimental animal numbers and characteristics used for auditory

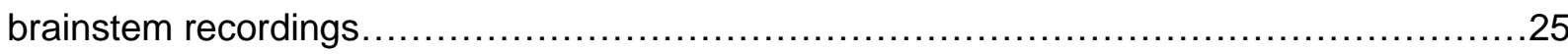

Table 3. Experimental animal numbers and characteristics used for PPI gap-detection........32

Table 4. Correlation between ABR wave and auditory station activity ........................53

Table 5. Comparison of amplitude and intensity ABR measurements between $\mathrm{MBP}^{+/+}$and

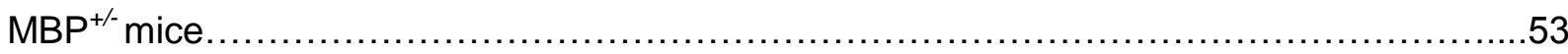




\section{Addendum}

Herewith I declare that I prepared the PhD Thesis "The role of oligodendrocytes in higherorder circuit functions" on my own and with no other sources and aids than quoted.

Sharlen Yared Moore Corona

Gottingen, 30.04.2018 


\section{Curriculum vitae}

\section{Sharlen Yared Moore Corona}

Max Planck Institute of Experimental Medicine

Department of Neurogenetics.

Hermann-Rein-Str. 3. 37075. Lower Saxony, Göttingen,

Germany.

(+49) 551-3899-765 | (+49) 176-8776-1579 | moore@em.mpg.de

Born in: Mexico City, Mexico | 25.03.1989

\section{EDUCATION}

05/2014-Present

PhD in Neurosciences

Thesis Project: 'The role of myelin in neuronal network function from a behavioral and electrophysiological perspective'.

Program: International Max Planck Research School for Neurosciences/Georg-AugustUniversität Göttingen, Germany. Cognitive Neurophysiology Lab, Department of Neurogenetics, Max Planck Institute of Experimental Medicine.

Thesis advisors: Dr. Livia de Hoz / Prof. Klaus-Armin Nave PhD.

$53 / 20$ Credits.

10/2012-03/2014 Master of Science in Neurosciences

Thesis Project: 'The role of myelin in sensory processing and interhemispheric information transfer'.

Program: International Max Planck Research School for Neurosciences/Georg-AugustUniversität Göttingen, Germany. Cognitive Neurophysiology Lab, Department of Neurogenetics, Max Planck Institute of Experimental Medicine.

Thesis advisors: Dr. Livia de Hoz / Prof. Klaus-Armin Nave PhD.

Final GPA: 2.01 (good).

08/2007-07/2012 Bachelor Degree in Basic Biomedical Research

Thesis Project: 'ROS generation and caspase- 3 activity induced by $\beta A-\mathrm{Cu}^{2+}$ complexes in cortical primary cultures'.

Molecular Neuropathology Department. Instituto de Fisiología Celular, Universidad Nacional Autónoma de México. Mexico City.

Thesis advisor: Dr. Ma. del Lourdes Massieu Trigo.

Final GPA: 9.11 out of 10 (very good).

$08-11 / 2011$

Qualification in Bioethics

Colegio de Bioética, A.C., Instituto de Fisiología Celular, Instituto de Investigaciones

Filosóficas, Universidad Nacional Autónoma de México, Mexico City.

08/2004- 06/2007 High School

Colegio Madrid A.C., Mexico City.

GPA: 9.19 out of 10 (very good)

\section{EXPERIENCE}

$05-06 / 2017$

Teaching assistant in Neurobiology of Hearing graduate school course

Neuroscience in Salamanca study abroad program from the University of Connecticut, USA. Organizers: Prof. Douglas L. Oliver PhD, and Prof. Dr. Manuel Sánchez Malmierca.

Institute of Neuroscience of Castilla y León, Salamanca, Spain. 
02-07/2016

09/2015

$08 / 2015$

07/2014

10/2013-03/2014

$05-07 / 2013$

03-05/2013

$01-03 / 2013$

08/2010-05/2012

08/2009-07/2010

08/2008- 07/2009
Lab rotation supervisor

Project: 'Assessment of interhemispheric synchronicity during sleep and paw preference behaviors in myelin mutant mice'

IMPRS program, Georg-August- University, Göttingen, Germany

International School for in vivo intracellular recordings (11 days)

The Edmond \& Lily Safra Center for Brain Sciences (ELSC),

Interuniversity Institute (IUI), Eilat, Israel.

Lab Internship (2 weeks)

To learn in vivo loose-cell patch clamp in the auditory cortex of mice.

Lab of Prof. Adi Mizrahi, ELSC-Hebrew University of Jerusalem, Israel.

Lab rotation supervisor

Project: 'Alternative methods for paw preference testing in pups'

Georg-August- University, Göttingen, Germany

\section{MSc Thesis}

Thesis Project: 'The role of myelin in sensory processing and interhemispheric information transfer'.

Cognitive Neurophysiology Lab. Department of Neurogenetics, Max Planck Institute of Experimental Medicine, Göttingen, Germany.

Thesis advisors: Dr. Livia de Hoz / Prof. Klaus-Armin Nave PhD

Thesis GPA: 1 (excellent).

\section{Lab rotation in Cognitive Neurophysiology Lab}

Project: 'Assessment of sound processing in mice with reduced myelin basic protein' Neurogenetics Department, Max Planck Institute of Experimental Medicine, Göttingen, Germany.

Supervisor: Dr. Livia de Hoz.

\section{Lab rotation in Glia Physiology Lab}

Project: 'Coupling of astrocytes in different brain regions'

Neurophysiology Department, Georg-August-Universität Göttingen, Germany.

Supervisors: Prof. Dr. Swen Hülsmann and Dr. Christian Schnell.

\section{Lab rotation in CNS Myelin Lab}

Project: 'Involvement of the immunoglobulin superfamily in neuron-glia interactions'

Cellular Neuroscience Department, Max Planck Institute of Experimental Medicine, Göttingen, Germany.

Supervisor: Prof. Dr. Mikael Simons and MSc. Tina Kling.

\section{Bachelor Thesis}

Thesis Project: 'ROS generation and caspase-3 activity induced by $\beta \mathrm{A}-\mathrm{Cu}^{2+}$ complexes in cortical primary cultures'.

Molecular Neuropathology Department. Instituto de Fisiología Celular, Universidad Nacional Autónoma de México. Mexico City.

Thesis advisor: Dr. Ma. del Lourdes Massieu Trigo.

\section{Lab rotation}

Project: 'ROS generation and caspase- 3 activity induced by $\beta A-\mathrm{Cu}^{2+}$ complexes in cortical primary cultures'.

Molecular Neuropathology Department. Instituto de Fisiología Celular, Universidad Nacional Autónoma de México.

Supervisor: Dr. Ma. del Lourdes Massieu Trigo.

\section{Lab rotation}

Project: 'Comparative analysis of the immune response to three PE_PGRS proteins from Mycobacterium tuberculosis'.

Immunology Department. Instituto de Investigaciones Biomédicas, Universidad Nacional Autónoma de México.

Supervisor: Dr. Clara Inés Espitia Pinzón. 
Project: 'Phenotipic characterization of Streptomyces coelicolor impaired in the protein glycosylation system'.

Molecular Biology and Biotechnology Department. Instituto de Investigaciones Biomédicas, Universidad Nacional Autónoma de México.

Supervisor: Dr. Luis Servín González.

\section{OTHER ACTIVITIES AND ADDITIONAL TRAINING}

$09 / 2014$

06-07/2007

$06 / 2005-08 / 2006$
Qualifying course in laboratory animals (1 week)

The course follows the recommendation of the FELASA (category B) and is certified by GV-SOLAS.

Max Planck Institute for Biophysical Chemistry, Göttingen, Germany.

\section{Student Representative of the Bachelor's Degree in Basic Biomedical Research}

Course: Applications, Uses and Handling of Laboratory Animals for Biomedical Research

MVZ. Atonatiu Edmundo Gómez Martínez

Facultad de Química, Universidad Nacional Autónoma de México.

Volunteer for adult literacy

Colegio Madrid, A.C., Mexico City.

\section{CONFERENCES AND MEETINGS}

$07 / 2017$

02/2017

$07 / 2016$

$05-06 / 2016$

05/2015
XIII European Meeting on Glial Cells in Health and Disease (4 days).

Poster presentation: 'Temporal sound processing in the auditory cortex is influenced by changes in myelin integrity'. Edinburgh, United Kingdom.

$12^{\text {th }}$ Göttingen Meeting of the German Neuroscience Society (4 days).

Poster presentation: 'Temporal sound processing in the auditory cortex is influenced by changes in myelin integrity'. Göttingen, Germany.

$40^{\text {th }}$ Annual MidWinter Meeting of the Association for Research in Otolaryngology (5 days)

Poster presentation: 'Temporal sound processing in the auditory cortex is influenced by both myelin integrity and oligodendrocyte-dependent metabolic support'. Baltimore, Maryland, USA.

FENS 2016 (4 days)

Poster presentation: 'Myelin integrity influences the establishment of behavioral laterality'. Copenhangen, Denmark.

Neurizons 2016: Speak your mind (4 days).

$7^{\text {th }}$ biannual neurosciences conference organized by the students of IMPRS for Neurosciences. Formed part of the organization committee.

Max Planck Institute for Biophysical Chemistry, Göttingen, Germany.

Neurizons 2015: Notes of an orchestrated brain (4 days).

$6^{\text {th }}$ biannual neurosciences conference organized by the students of IMPRS for Neurosciences. Formed part of the organization committee and poster presentation: 'Myelin integrity influences auditory temporal processing and the establishment of behavioral laterality'

Max Planck Institute for Biophysical Chemistry, Göttingen, Germany. 


\section{Science and Innovation Week 2010}

Poster presentation: 'ROS generation and caspase- 3 activity induced by $\beta \mathrm{A}-\mathrm{Cu}^{2+}$ complexes in cortical primary cultures'. Instituto de Ciencia y Tecnología del Distrito Federal. Palacio de Minería, Mexico City.

$11 / 2010$

\section{National Congress of Biochemistry (6 days).}

Poster presentation: 'ROS generation and caspase-3 activity induced by $\mathrm{BA}^{-\mathrm{Cu}^{2+}}$ complexes in cortical primary cultures'. Sociedad Mexicana de Bioquímica, A.C.

Tuxtla Gutiérrez, Chiapas, México.

\section{AWARDS, SCHOLARSHIPS AND MEMBERSHIPS}

10/2014-Present

09/2017

05/2017

$02 / 2017$

09/2016-Present

10/2015-Present

$08 / 2015$

10/2012-09/2013

$11 / 2011$

$08 / 2011$

03/2011

$11 / 2010$

$08-12 / 2010$
Scholarship for PhD studies from the National Council of Science and Technology of Mexico in cooperation with the German Academic Exchange Service (CONACyT-DAAD ID: 91553155 . Programme: 50015952).

2017 SfN Trainee Professional Development Award

Award to attend the 2017 SfN meeting in Washington DC, and be part of a series of professional development workshops for early career researchers.

\section{Society for Neurosciences Member}

ARO Travel Award

Travel Award to attend the 2017 Association for Research in Otolaryngology MidWinter Meeting.

\section{Association for Research in Otolaryngology Member}

\section{German Neuroscience Society Member}

\section{Boehringer Ingelheim Fonds Travel Grant}

Travel grant from the Boehringer Ingelheim Foundation, used for a lab internship and an electrophysiology course in Israel.

\section{Stipend of the Excellence Foundation for the Promotion of the Max Planck Society \\ 18 Master's scholarships awarded from more than 200 applicants internationally.}

Thesis realization scholarship from the Ministry of Public Education

To support bachelor students during thesis writing. SEP, Mexico.

Academic Merit Diploma 2010

For being on the first three places of the Bachelor's Degree in Basic Biomedical Research during 2010. Universidad Nacional Autónoma de México.

\section{Academic Merit Diploma 2008-2009}

For being on the first three places of the Bachelor's Degree in Basic Biomedical Research during 2008-2009. Universidad Nacional Autónoma de México.

Social Service scholarship from the Ministry of Public Education For the realization of social service during the bachelor studies. SEP, Mexico.

Federal District's Science and Technology Institute scholarship Mexico, (ICyTDF PIFUTP08-161). 
Curriculum vitae

03-06/2010

Project Support Program for Research and Technological Innovation scholarship Mexico, (UNAM-PAPIIT IN211710).

$08 / 2009$

National Council of Science and Technology scholarship to support undergraduate students

CONACyT, Mexico (scholarship No. 102775).

\section{LANGUAGES}

Spanish: native speaker | English: fluent (7/9 IELTS in 2012) | French: basic (2 years of private lessons) | German: basic (A2).

10/2012- 2014

German language courses

Lektorat Deutsch als Fremdsprache, Georg-August Universität Göttingen, Germany. 